\title{
Experimental verification of 4D Monte Carlo simulations of dose delivery to moving and deforming anatomies
}

by

Sara Gholampourkashi

A thesis submitted to the Faculty of Graduate and Postdoctoral Affairs in partial fulfillment of the requirements for the degree of

Doctor of Philosophy

in

Physics (Medical Physics)

Ottawa-Carleton Institute for Physics

Department of Physics

Carleton University

Ottawa, Ontario

C 2019 Sara Gholampourkashi 


\begin{abstract}
The aim of this work was to validate a 4D Monte Carlo (MC) dose calculation tool (4DdefDOSXYZnrc/EGSnrc) by comparing dose calculations with measurements in rigid and deforming anatomies. This 4DMC approach, that is based on the voxel warping method, calculates the cumulative dose by including continuous motion/deformation of anatomy and motion of linac components. We present how motion recorded during dose delivery combined with deformation/displacement vectors and delivery log files can be used to incorporate motion of the anatomy and treatment units into simulations. Dose deliveries were performed on an Elekta Infinity linac with Agility MLC for static and volumetric modulated arc therapy treatments on the stationary and breathing states of the phantoms. The dose was measured with EBT3 film (point dose and dose profile) and the RADPOS 4D dosimetry system (point dose).
\end{abstract}

Preliminary validations were done using a phantom with a rigidly moving lung insert. To evaluate the performance of $4 \mathrm{DMC}$ simulations for more realistic conditions a programmable deformable tissue-equivalent lung phantom was developed. The lung inserts in both phantoms served the purpose of holding the film as well as a spherical tumor to hold RADPOS. RADPOS detectors were also placed outside the tumor in the deformable phantom. Sinusoidal (both phantoms) and irregular (deformable phantom) respiratory traces were tested. Point dose agreements between simulations and measurements were within $2 \sigma$ of experimental and/or positional/dose reading uncertainties. The agreement between dose profiles from measurements and simulations was determined to be within $2 \% / 2 \mathrm{~mm}$. 
We also present Monte Carlo modeling of the Elekta linac used in this work. A detailed model was created in BEAMnrc/EGSnrc and model parameters were adjusted through appropriate measurements. We demonstrate how using a simple virtual source model of a linac can help study the sensitivity of the isocentric photon fluence to several beam parameters for small radiation fields. Finally, we present how we can use MC simulations and the virtual source model to explain dose discrepancies observed between film measurements and calculations obtained from the Monaco treatment planning system. The results show that proper model parameters can heavily impact the accuracy of dose calculations of small fields. 


\section{Acknowledgments}

I would like to express my special appreciation and thanks to my supervisors Dr. Joanna Cygler and Dr. Emily Heath for their invaluable support. Their guidance and encouragement at all stages of my PhD made it a wonderful journey. It was their patience and motivation that allowed me to develop my skills and grow both as a person and as a research scientist.

I would like to thank Dr. Jason Bélec with whom I worked very closely during Chapter 4 of my thesis. I also had many helpful conversations with him regarding several aspects of my research work.

Special gratitude to Bernie Lavigne for the exceptional work with the deformable phantom development. The successful design and development of our novel phantom would not be possible if it was not for his collaboration.

Thank you to Miro Vujicic for all help and discussions during initial stages of this project.

I would like to thank Steve Andrusyk and his team at the CT-Sim department of the Ottawa Hospital Cancer Centre for their help with CT scans of our phantoms.

I would like to give my thanks to Pat Lacasse for her help with treatment planning aspects of the project. Thanks to Chris Lambert for his help with some aspects of our latest experiments. I would also like to acknowledge Jason Smale of Elekta for helping us with delivery $\log$ files. 
I would like to thank my nominal co-supervisor Dr. Manuella Vincter for her help and advice throughout my $\mathrm{PhD}$.

Finally, thanks to my family and friends for their encouragement and support during the years of my $\mathrm{PhD}$ work. 


\section{Statement of originality}

This thesis describes the results of the author's research conducted primarily at the Ottawa Hospital Cancer Centre during the course of the $\mathrm{PhD}$ program of Carleton University. Most of these results have been published or will be submitted in on of the Medical Physics, Physica Medica, Journal of Applied Clinical Medical Physics or Physics in Medicine and Biology journals and have been presented at both national and international conferences. The details of where the results of these publications may be found in the thesis, along with the author's contributions, are provided below:

I. S. Gholampourkashi, J.E. Cygler, J. Belec, M. Vujicic and E. Heath, Monte Carlo and analytic modeling of an Elekta Infinity linac with Agility MLC: Investigating the significance of accurate model parameters for small radiation fields, J. Appl. Clin. Med. Phys., doi:10.1002/acm2.12485 (2018).

- The results of this paper are described in Chapter 4.

- The author performed parts of the measurements and parts of the Monte Carlo simulations in collaboration with co-authors (Dr. Jason Bélec and Miro Vujicic) and performed the remainder of the Monte Carlo simulations, data analysis, prepared and revised the manuscript for publication under the supervision of Dr. Joanna Cygler and Dr. Emily Heath. The analytic model was initially developed by Dr. Jason Belec and the author modified the model to improve the accuracy, determined appropriate model parameters and studied their impact on the performance of the model. 
- The results of this paper were presented by the author as an e-poster at the 2017 AAPM conference and select results were presented as posters at the 2015 ESTRO and AAPM conferences.

II. S. Gholampourkashi, M. Vujicic, J. Belec, J.E. Cygler and E. Heath, Experimental verification of 4D Monte Carlo simulations of dose delivery to a moving anatomy, Med. Phys. 44(1), 299-310 (2017).

- The results of this paper constitute parts of Chapter 5 and Chapter 6 that discuss the characteristics of the Quasar phantom and experimental verifications using this phantom, respectively.

- The author performed all of the measurements, Monte Carlo simulations, data analysis and prepared the manuscript for publication under the supervision of Dr. Joanna Cygler and Dr. Emily Heath. Final edits and revisions were made in collaboration with co-authors.

- The results from this paper were presented by the author at the 2015 World Congress on Medical Physics and Biomedical Engineering and 2016 AAPM conferences as an oral presentation and at the 2016 IMNO conference as a poster. They have also been presented at the 2016 ICCR conference and as a poster at the 2016 ESTRO conference.

III. S. Gholampourkashi, J.E. Cygler, B. Lavigne and E. Heath, Development of a deformable phantom for experimental verification of 4D Monte Carlo simulations in a deforming anatomy, Physica Medica, 51, 81-90 (2018). 
- The results of this paper constitute parts of Chapter 5 and Chapter 6 that discuss the design and verification of the deformable phantom as well as experimental verifications using this phantom, respectively.

- Development of the phantom was done in close collaboration with Bernie Lavigne. The author performed all of the measurements, Monte Carlo simulations, data analysis and prepared the manuscript for publication under the supervision of Dr. Joanna Cygler and Dr. Emily Heath.

- The results of this paper were presented by the author at the 2017 COMP and 2018 AAPM conferences as an oral presentation and at the 2018 ESTRO conference as a poster discussion. They have also been presented as an oral presentation at the 2017 Monte Carlo Methods and Applications (MCMA) conference.

\section{Manuscripts in preparation}

S. Gholampourkashi, J.E. Cygler, B. Lavigne and E. Heath, Validation of 4D Monte Carlo dose calculations using a programmable deformable lung phantom. Under review with Physics in Medicine and Biology: Submitted Jan 2019.

- The results of this paper constitute parts of Chapter 5 that describe modifications to the deformable phantom to a programmable phantom. They also constitute parts of Chapter 6 that describe experimental verifications for which this phantom was utilized.

- Modifications to the phantom were done in collaboration with Bernie Lavigne. The author performed all the programming work related to the 
phantom, as well as all the measurements, Monte Caro simulations, data analysis and prepared the manuscript for publication under the supervision of Dr. Joanna Cygler and Dr. Emily Heath.

- The results of this paper have been submitted as an abstract to the 2019 ESTRO and joint ICCR-MCMA conferences. 


\section{List of abbreviations}

3D-CRT

4DMC

A-P

BDF

BEV

C/S

CM

CT

CTV

DICOM

DIM

DIR

DTA

DVF

EBRT

ECUT

EGS

EMCM

EOE

EOI

FSB
Three-dimensional conformation radiation therapy

four-dimensional Monte Carlo

Anterior-posterior

Binary data format

Beam's eye view

Convolution/superposition

Component module

Computed tomography

Clinical target volume

Digital imaging and communications in medicine

Dose interpolation method

Deformable image registration

Distance-to-agreement

Deformation vector field

External beam radiation therapy

Electron cut-off energy

Electron-Gamma-Shower

Energy mass congruent mapping

End-of-exhale

End-of-inhale

Forced shallow breathing 
FWHM

GTV

HU

IMRT

ITV

LBROT

LCPE

LED

Linac

L-R

MC

MLC

MOSFET

mrad

MRI

MU

NTP

OAR

OAR

PCUT

PDD

PDF

P-P
Full-width-at-half-maximum

Gross target volume

Hounsfield unit

Intensity modulated radiation therapy

Internal target volume

Leaf bank rotation

Lateral charged particle equilibrium

Light emitting diode

Linear accelerator

Left-right

Monte Carlo

Multi-leaf collimator

Metal-oxide semiconductor field effect transmitter

Milliradians

Magnetic resonance imaging

Monitor units

Network time protocol

Organs at risk

Off-axis ratio

Photon cut-off energy

Percentage-depth-dose

Probability distribution function

Peak-to-Peak 
PPS

PS

PTV

RDF

RED

ROI

SAD

SBRT

S-I

SSD

TCPE

TLD

TPS

VMAT

VSM

VWM

XVMC
Position probability sampling

Phase space

Planning target volume

Relative dose factor

Relative electron density

Region of Interest

Source to axis distance

Stereotactic body radiation therapy

Superior-inferior

Source to surface distance

Transient charged particle equilibrium

Thermoluminescent dosimeter

Treatment planning system

Volumetric modulated arc radiation therapy

Virtual source model

Voxel warping method

X-ray voxel Monte Carlo 


\section{Table of Contents}

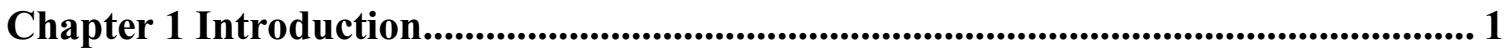

1.1 Radiation therapy and cancer treatment............................................................ 1

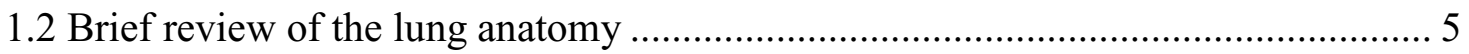

1.3 Impact of patient respiratory motion on radiotherapy treatment delivery ............... 6

1.4 Management of respiratory motion in radiotherapy ........................................... 9

1.5 Incorporating respiratory motion in dose calculation ........................................ 10

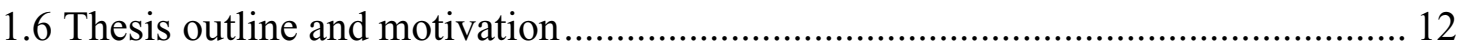

Chapter 2 Dose measurements .............................................................................. 14

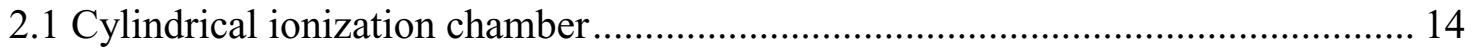

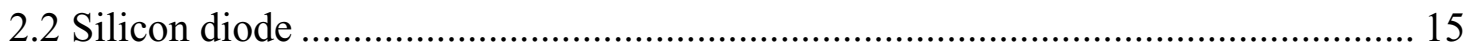

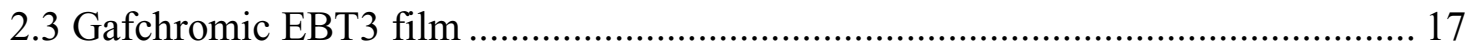

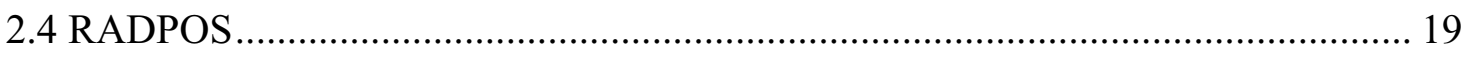

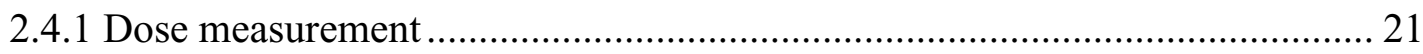

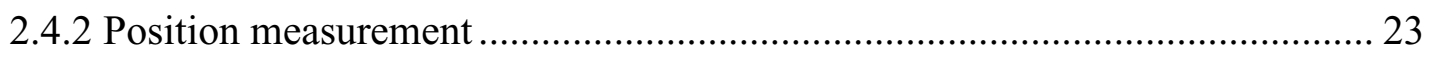

Chapter 3 Monte Carlo user codes................................................................................. 25

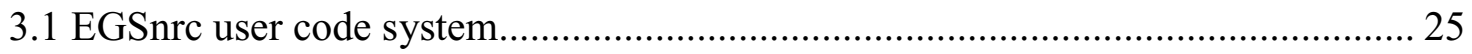

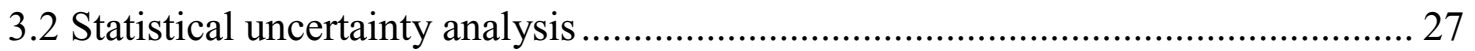

3.3 BEAMnrc, DOSXYZnrc and 4DdefDOSXYZnrc user codes............................. 28

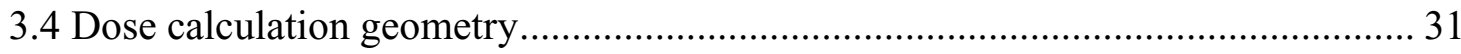

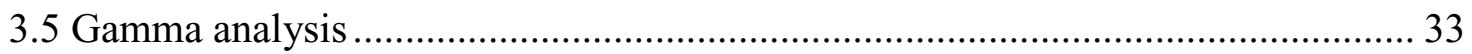

Chapter 4 Monte Carlo and analytic modeling of an Elekta Infinity linac with Agility MLC: Investigating the significance of accurate model parameters for small

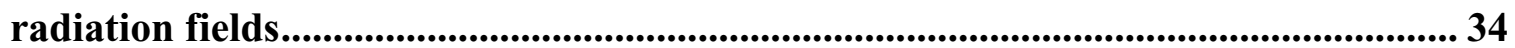

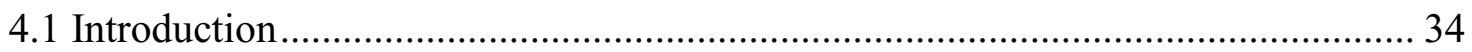

4.1.1 Monte Carlo beam models .......................................................................... 34

4.1.2 Virtual source models ................................................................................. 35 


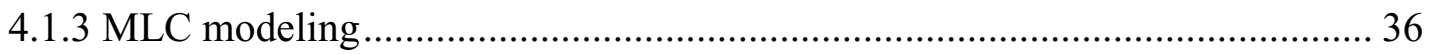

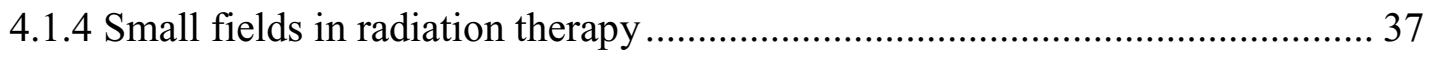

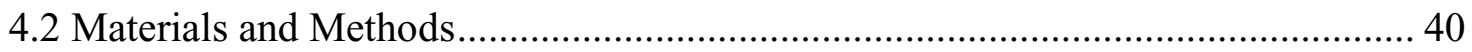

4.2.1 BEAMnrc model of an Elekta Infinity linear accelerator .................................. 40

4.2.2 Analytic and virtual source model of the Elekta Infinity linac .........................46

4.2.3 Impact of analytic model parameters on the fluence at the isocenter plane .... 49

4.2.4 Comparison of Monaco and EGSnrc dose calculations.................................... 49

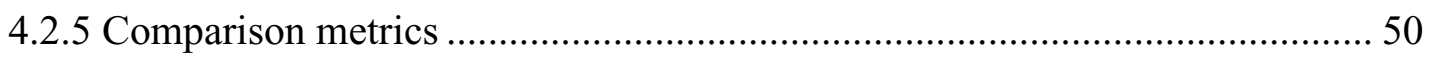

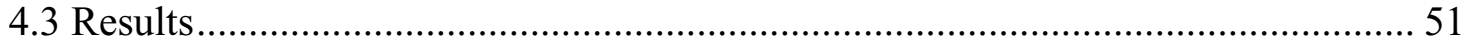

4.3.1 BEAMnrc model of an Elekta Infinity linear accelerator ................................. 51

4.3.2 Analytic and virtual source model of the Elekta Infinity linac..........................58

4.3.3 Impact of analytic model parameters on the fluence at the isocenter plane .... 58

4.3.4 Comparison of Monaco and EGSnrc dose calculations...................................... 61

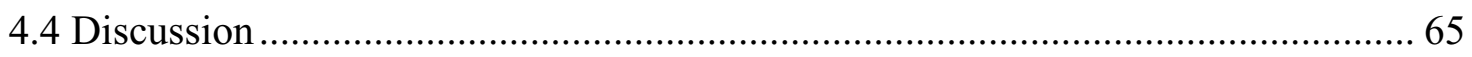

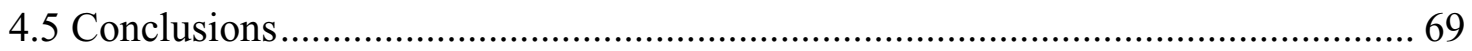

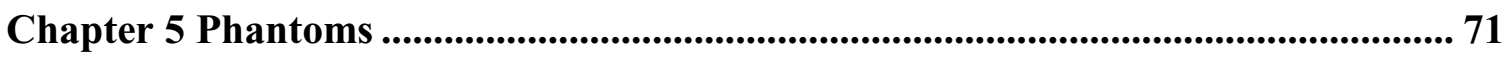

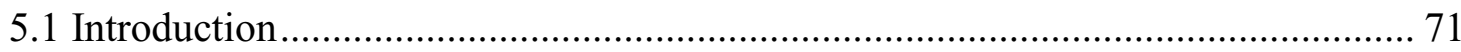

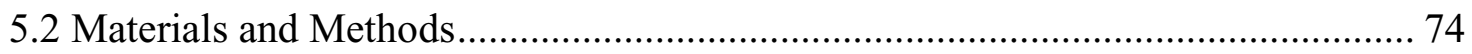

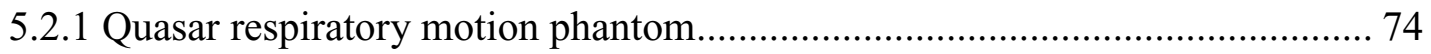

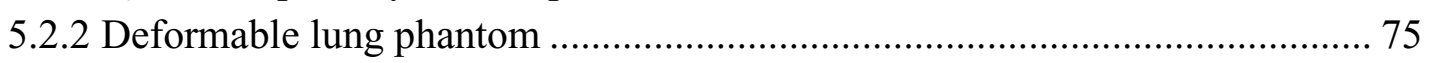

5.2.3 Deformable programmable lung phantom ...................................................... 78

5.2.3.1 Motion assessment and reproducibility ................................................. 79

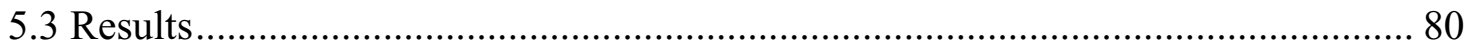

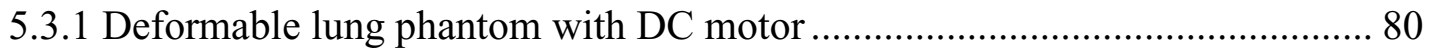

5.3.1.1 Motion assessment and reproducibility .................................................. 81

5.3.2 Motion assessment and reproducibility of the deformable programmable lung

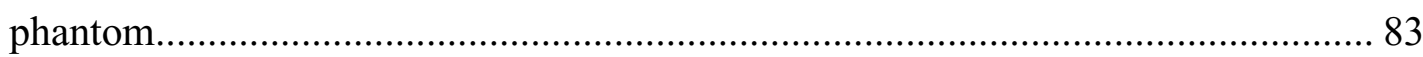

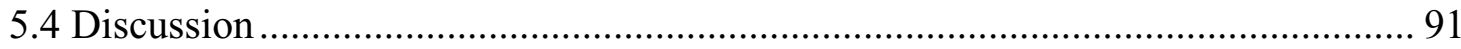

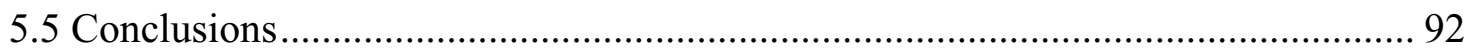

Chapter 6 Experimental verification of 4D Monte Carlo simulations of dose delivery to moving/deforming phantoms............................................................................................. 93

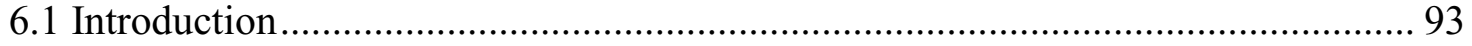

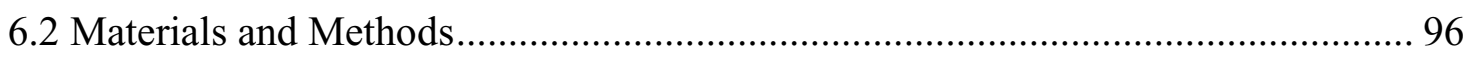

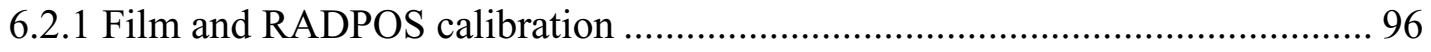

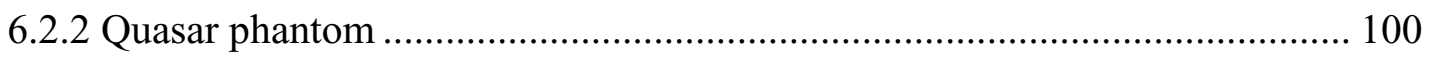




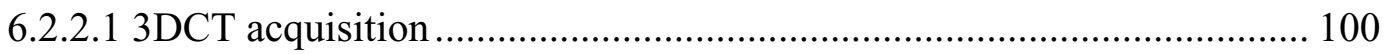

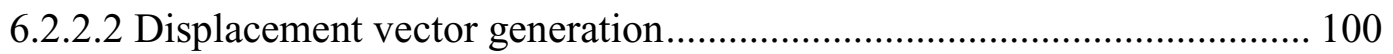

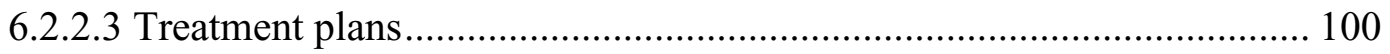

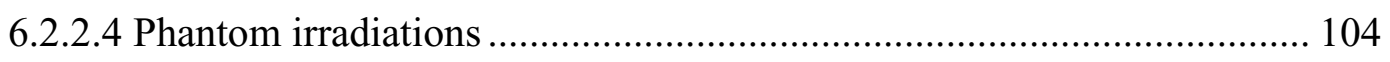

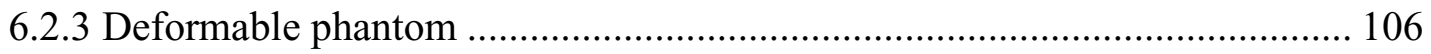

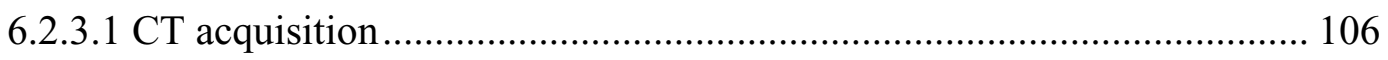

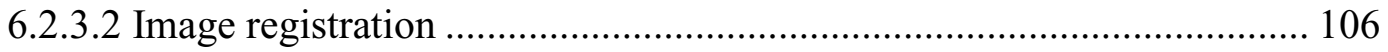

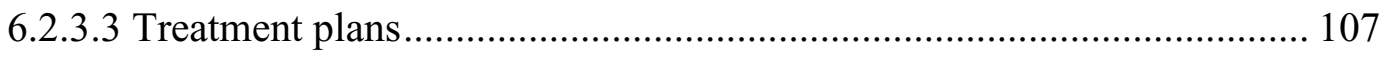

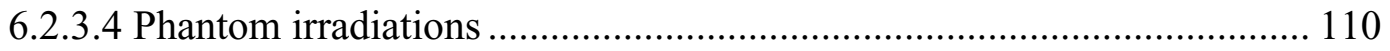

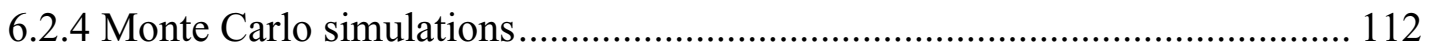

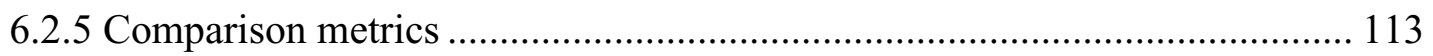

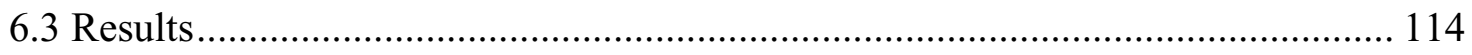

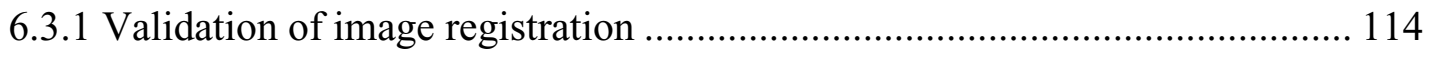

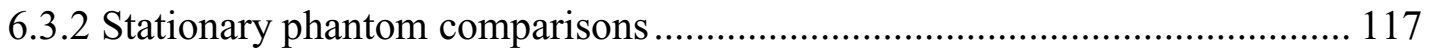

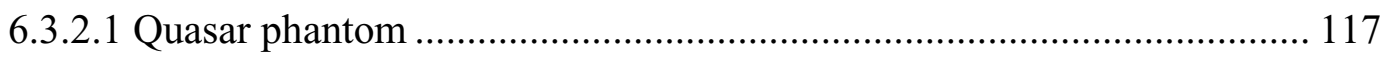

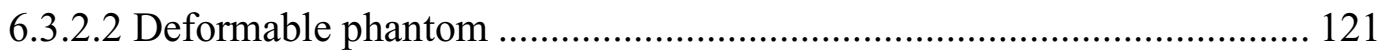

6.3.3 Moving and deforming phantom comparisons ............................................. 124

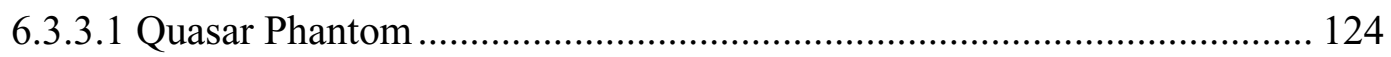

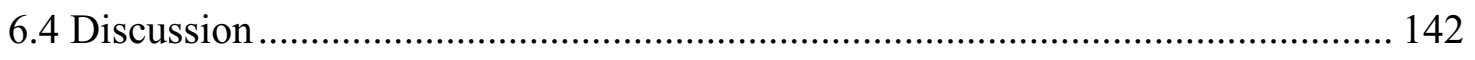

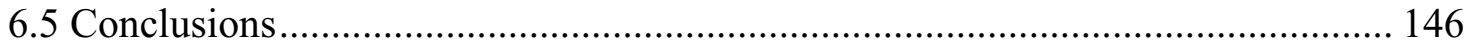

Chapter 7 Summary and Outlook .......................................................................................... 148

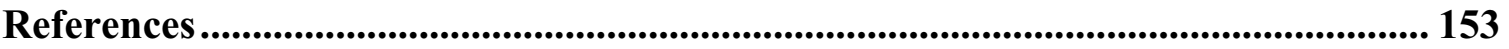




\section{List of Figures}

Figure 1.1 A conventional linac - Elekta Infinity with typical components including gantry, treatment head and treatment couch (left) with Agility MLC (right).

Figure 1.2 Delineations of GTV, CTV, ITV, PTV and OAR used throughout treatment planning process. Adapted from the ICRU reports $50^{7}$ and $62^{8}$.................................... 3

Figure 1.3 Anatomy of lung showing the five lobes on the left and right lung................. 5

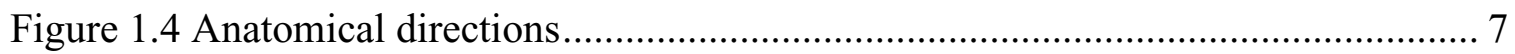

Figure 1.5 Dose profiles for stationary (solid blue) and moving/breathing (dashed red) states of the anatomy. Respiratory motion results in a broader penumbra and blurs dose

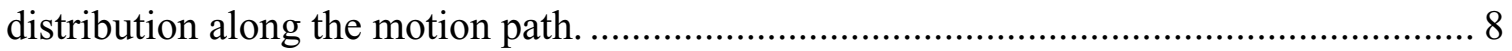

Figure 1.6 Subtracted doses for dose distributions calculated in exhale and inhale respiratory phases with the same treatment plan. Without the localized dose deformations, the differences shown in (a) inhale-exhale and (b) exhale-inhale should be zero. Dose differences are normalized to the calculated dose in inhale. Adapted with permission from Bortfeld et al., 2004 20

Figure 1.7 Interplay effect between the target volume (red star) and multi-leaf collimator. The target moves superior-inferior while leaves sweep from left to right. The dose received by the target volume is a function of the time it is covered by the leaves and thus depends on the relation between motion of the target and motion of the leaves. The target is receiving a higher dose in the second delivery (bottom) than in the first (top).

Figure 1.8 The process of 4D dose calculation. Dose distribution is calculated on multiple respiratory phases and then mapped back to a reference phase to construct the cumulative dose distribution. Obtained with permission from Keall et al., $2005^{61}$. 11

Figure 2.1 Schematic of a cylindrical ionization chamber (right) connected to an electrometer (left) 14

Figure 2.2 Schematic of construction of a silicon photon diode dosimeter. Adapted from Cranmer-Sargison et al. ${ }^{71}$ 16

Figure 2.3 Structure of Gafchromic EBT3 film 18

Figure 2.4 Calibration curve for EBT3 film to convert change in the optical density to absorbed dose. The maximum irradiated calibration dose is $845.7 \mathrm{cGy}$. 
Figure 2.5 An overview of the RADPOS 4D dosimetry system with its components including MOSFET reader, 3D-Guidance tracker, pre-amplifier, transmitter and probe. MOSFET reader and 3D-Guidance tracker are connected to a host PC that runs the RADPOS software.

Figure 2.6 The RADPOS probe. The microMOSFET and position sensors are separated by $8 \mathrm{~mm}$. 20

Figure 2.7 Schematic of the structure of a p-type MOSFET (in the read-out mode) used in dosimetry. Adapted from Soubra et al., 199475.

Figure 2.8 The RADPOS DC magnetic field transmitter and its coordinate system. The Cartesian coordinates correspond to the left-right $(\mathrm{X})$, superior-inferior $(\mathrm{Y})$ and anteriorposterior $(\mathrm{Z})$ of the patient. 23

Figure 3.1 Structure of the EGSnrc code system with user codes. Adapted from Kawarkow et al., $2013^{77}$ 26

Figure 3.2 Diagram showing how the 4DdefDOSXYZnrc user code works based on the voxel warping approach. The input parameters needed to initialize the simulation are shown on the left-hand illustration. The respiratory motion trace functions as the scaling factor for deformation vectors and could be between 0 and 1 or -1 and +1 . The diagram on the right-hand shows a summary of how the dose is calculated during simulation. 30

Figure 4.1 Illustration of source occlusion and the resultant dose profile. If the field size is large enough (left), beam source is fully viewed at the isocenter plane and the resulting dose profile measures the correct field size. For very small field sizes (right), beam source is partially viewed at the isocenter plane which yields a widened dose profile that measures an overestimated field size due to a larger FWHM. Adapted from IPEM Report 103, $2010^{123}$ and Das et al., $2008^{119}$ 38

Figure 4.2 BEAMnrc preview of the Elekta Infinity linac model with Agility MLC showing the various components. 40

Figure 4.3 In-plane view of MLC leaf banks with defocusing (top left) vs focused with tongue $\&$ groove mechanism (bottom left) to reduce inter-leaf leakage. Zoomed views are also shown (top right and bottom right).

Figure 4.4 BEV of the fields constructed to evaluate the LBROT value. The fields consist of a small field size (1-open leaf) to verify leaf bank rotation and one larger field size (5open leaves) for dosimetric verification. 44 
Figure 4.5 Primary (top) and Secondary (bottom) virtual photon sources used in the analytic model. The primary and secondary photon sources are placed at $Z=1.1 \mathrm{~cm}$ and $Z$ $=15.9 \mathrm{~cm}$ from the reference plane $(\mathrm{Z}=0 \mathrm{~cm})$, respectively.

Figure 4.6 Ray diagram illustrating the photon fluence calculation process of the analytic model. The fluence at each point along the in-line position on the isocenter plane is the integral of the source. The source boundaries are shown by the photon rays tracing from the isocenter to the source plane. 48

Figure 4.7 Comparison of the measured and calculated PDD curves for a $\quad 5 \times 5 \mathrm{~cm}^{2}$ field size at $\mathrm{SSD}=100 \mathrm{~cm}$ normalized at $10 \mathrm{~cm}$ depth (top) and percent dose differences for calculated point doses against measurements (bottom). Error bars represent statistical uncertainty from MC simulations $(0.1 \%)$. 52

Figure 4.8 Comparison of the measured and calculated (a) cross-line and (b) in-line profiles for various field sizes at $5 \mathrm{~cm}$ depth and $100 \mathrm{~cm} \mathrm{SSD.}$ 53

Figure 4.9 Comparison of the measured and calculated relative output factors (RDFs) for various field sizes at $5 \mathrm{~cm}$ depth and $100 \mathrm{~cm}$ SSD. 54

Figure 4.10 Comparison of dose profiles between film measurements (solid blue) and MC simulations (dotted red) for the field shown in Figure 4.4 for LBROT values of (a) 0 , (b) 6, (c) 9 and (d) $12 \mathrm{mrad}$. The best fit parameter was found to be LBROT $=9 \mathrm{mrad}$...... 55

Figure 4.11 Variation of leaf transmission by increasing the inter-leaf air gap. All transmission values are normalized to the transmission corresponding to the nominal interleaf air gap. 56

Figure 4.12 Photon fluence at the isocenter plane from MC simulations and analytic model calculations for LBROT $=9 \mathrm{mrad}$. Fluence curves are normalized to their integral so that the integral of the resultant curve is equal to 1 . 58

Figure 4.13 Impact of changes in (a) Leaf bank rotation (normalized to nominal value of leaf bank rotation or LBROT $=9 \mathrm{mrad}$ ), (b) Leaf attenuation (normalized to full MLC leaf attenuation) and (c) Primary source size (normalized to nominal source size of $1 \mathrm{~mm}$ ) on the relative fluence integral, maximum fluence and average DTA of the fluence in analytic model 60

Figure 4.14 Comparison of Monaco calculations against (a) film measurements and (b) EGSnrc simulations for the field shown in Figure 4.4.

Figure 4.15 Comparison between Monaco calculations and EGSnrc simulations with modified parameters: $\mathrm{FWHM}=2 \mathrm{~mm}$ in the in-line position and inclusion of tongue \& 
groove. Dose differences were reduced to approximately $1 \%$ at the maxima and $2 \%$ at the minima.

Figure 5.1 (a) The Quasar respiratory motion phantom, (b) wooden lung insert in two halves with the groove to insert RADPOS probe and (c) Solid Water tumor. 75

Figure 5.2 (a) The deformable lung phantom with different parts including the flexible foam, plug, piston and DC motor, (b) Cylindrical plug fixes inside the foam, (c) silicon rubber tumor inside the plug and (d) Lucite beads injected throughout the phantom...... 76

Figure 5.3 RADPOS detectors on the top surface (top), inside (middle) and bottom surface (bottom) of the plug. 77

Figure 5.4 Deformable lung phantom with programmable servo motor to simulate realistic respiratory motion profiles (top) and Scotch Yoke mechanism to transform the rotational motion of the motor to linear motion at the piston (bottom). 78

Figure 5.5 Varying levels of hysteresis shown by S-I vs. A-P motion for landmarks (white circles) closest to the piston (top row), middle of the insert (middle row) and furthest from the piston (bottom row). As expected, landmarks closest to the piston show the largest amounts of hysteresis. The data were acquired while the phantom moved with a sinusoidal respiratory motion (period of $3 \mathrm{~s} ; 4 \mathrm{~cm}$ P-P amplitude at the piston). 81

Figure 5.6 Range of S-I motion measured by RADPOS detectors at the tumor (top), top (middle) and bottom (bottom) plug surfaces vs period of sinusoidal motion. Average P-P amplitude values and their reproducibility (combined standard deviations for one amplitude is the square root of the sum of the square of individual standard deviations) are shown on the plots. 84

Figure 5.7 Range of A-P motion measured by RADPOS detectors at the tumor (top), top (middle) and bottom (bottom) plug surfaces vs period of sinusoidal motion. Average P-P amplitude values and their reproducibility (combined standard deviations for one amplitude is the square root of the sum of the square of individual standard deviations) are shown on the plots. 85

Figure 5.8 Range of L-R motion measured by RADPOS detectors at the tumor (top), top (middle) and bottom (bottom) plug surfaces vs period of sinusoidal motion. Average P-P amplitude values and their reproducibility (combined standard deviations for one amplitude is the square root of the sum of the square of individual standard deviations) are shown on the plots. 86

Figure 5.9 Comparison of the tumor (orange dots) and diaphragm (green line) motion traces for respiratory motion profiles that are (a) typical, (b) highly irregular and (c) show large 
hysteresis between the tumor and diaphragm motion at a P-P diaphragm amplitude of 3 $\mathrm{cm}$.

Figure 5.10 Correlation curves between motion of the tumor and diaphragm for P-P diaphragm amplitude of $3 \mathrm{~cm}$. Correlations are shown in the form of hysteresis plots for sinusoidal motion profiles with periods of (a) $4 \mathrm{~s}$, (b) $7 \mathrm{~s}$ as well as (c) typical (Figure 5.9 (a)) and (d) large hysteresis between the tumor and diaphragm motion (Figure 5.9 (c)) respiratory motion profiles.

Figure 6.1 Measurement setup used for film and RADPOS calibration. Film and RADPOS were placed at $5 \mathrm{~cm}$ depth of the Solid Water phantom while the ion chamber was at $10 \mathrm{~cm}$ at an SSD setup of $100 \mathrm{~cm}$.

Figure 6.2 Static $4 \times 4 \mathrm{~cm}^{2}$ square plan: dose distributions from XiO on (a) axial, (b) coronal, and (c) sagittal planes

Figure 6.3 VMAT plan: dose distributions from Monaco on (a) axial, (b) coronal, and (c) sagittal planes.

Figure 6.4 Setup for Quasar phantom irradiations such that the cylindrical insert moved in the S-I direction (left) and center of the tumor inside the insert was aligned with the beam isocenter (right). 104

Figure 6.5 Film and RADPOS inside the lung insert of the Quasar phantom. RADPOS was placed inside a special groove, and film was taped on top of the RADPOS probe........ 105

Figure 6.6 Static $3 \times 3 \mathrm{~cm}^{2}$ square plan: dose distribution from Monaco on (a) coronal, (b) sagittal and (c) axial planes. 108

Figure 6.7 VMAT plan: dose distribution from Monaco on (a) coronal, (b) sagittal and (c) axial planes.

Figure 6.8 Setup for deformable phantom irradiations such that the piston was in the superior side of the couch and center of the tumor inside the phantom was aligned with the beam isocenter. 111

Figure 6.9 Film and RADPOS inside the plug to measure point dose (tumor center) and dose profile along the S-I direction. RADPOS is fixed inside an embedded groove and film is taped on top of RADPOS probe. 112

Figure 6.10 Coronal view of the phantom in Velocity: (a) non-deformed EOE (gray) overlaid on EOI (pink), (b) deformed EOE overlaid on EOI. The red rectangular area shows the selected region of interest (ROI) for image registration. 115 
Figure 6.11. Visual evaluation of the deformable image registration on coronal view: (a) deformed EOE subtracted from EOI and (b) Jacobian of the registration.

Figure 6.12 Comparison of dose profiles for $4 \times 4 \mathrm{~cm}^{2}$ (top) and VMAT (bottom) beam deliveries on the stationary Quasar phantom along the S-I direction. 118

Figure 6.13 Comparison of 2D dose distributions (top row) and gamma comparison maps (bottom row) from film measurements and MC simulations for $4 \times 4 \mathrm{~cm}^{2}$ (left) and VMAT (right) beam deliveries on the stationary Quasar phantom. Gamma comparison maps were generated using a threshold of $50 \% \mathrm{D}_{\max }$

Figure 6.14 Comparison of dose profiles for $3 \times 3 \mathrm{~cm}^{2}$ (top) and VMAT (bottom) beam deliveries on the stationary deformable phantom along the S-I direction.

Figure 6.15 Comparison of dose profiles for $4 \times 4 \mathrm{~cm}^{2}$ (top) and VMAT (bottom) beam deliveries on the breathing Quasar phantom along the S-I direction.

Figure 6.16 Comparison of 2D dose distributions (top row) and gamma comparison maps (bottom row) from film measurements and MC simulations for $4 \times 4 \mathrm{~cm}^{2}$ (left) and VMAT (right) beam deliveries on the breathing Quasar phantom. Gamma comparison maps were generated using a threshold of $50 \% \mathrm{D}_{\max }$

Figure 6.17 Comparison of dose profiles for $3 \times 3 \mathrm{~cm}^{2}$ (top) and VMAT (bottom) beam deliveries on the breathing deformable phantom along the S-I direction, for the sinusoidal breathing motion.

Figure 6.18 Comparison of dose profiles for $3 \times 3 \mathrm{~cm}^{2}$ (top) and VMAT (bottom) beam deliveries on the breathing deformable phantom along the S-I direction, for the typical breathing motion shown in Figure 5.9 (a)

Figure 6.19 Comparison of dose profiles for $3 \times 3 \mathrm{~cm}^{2}$ (top) and VMAT (bottom) beam deliveries on the breathing deformable phantom along the S-I direction, for the highly irregular breathing motion shown in Figure 5.9 (b). 131

Figure 6.20 Comparison of dose profiles for $3 \times 3 \mathrm{~cm}^{2}$ (top) and VMAT (bottom) beam deliveries on the breathing deformable phantom along the S-I direction, for the breathing motion with large hysteresis between the tumor and diaphragm motion shown in Figure 5.9 (c). 132

Figure 6.21 Fraction of the time spent in each breathing phase during $3 \times 3 \mathrm{~cm}^{2}$ (top) and VMAT (bottom) beam deliveries on the deforming phantom for three irradiations with the typical respiratory motion profile shown in Figure 5.9 (a). Breathing phase is the RADPOS normalized displacement that was recorded during beam deliveries. 139 
Figure 6.22 Fraction of the time spent in each breathing phase during $3 \times 3 \mathrm{~cm}^{2}$ (top) and VMAT (bottom) beam deliveries on the deforming phantom for three irradiations with the highly irregular respiratory motion profile shown in Figure 5.9 (b). Breathing phase is the RADPOS normalized displacement that was recorded during beam deliveries...... 140 


\section{List of Tables}

Table 3.1 Calibration data for the Big Bore helical CT scanner to map CT numbers (HU)

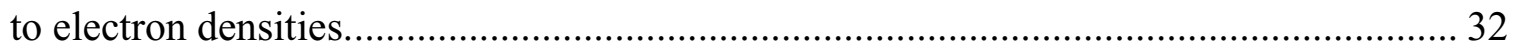

Table 4.1 Mean percentage dose differences at the maxima and minima as well as average DTA (left and right) values at penumbra region from film measurements and MC simulations for different LBROT values. Uncertainties are statistical uncertainties associated with dose values at different maxima and minima from the profiles. 55

Table 4.2 Derived parameters of the Infinity linac model with their uncertainties. 57

Table 4.3 The composition of the leaf as provided by manufacturer and adjusted in the MC beam model.

Table 4.4 Mean percentage dose differences at the maxima and minima as well as average DTA (left and right) values at penumbra region from Monaco calculations against film measurements and EGSnrc simulations. Uncertainties are statistical uncertainties associated to dose values at different maxima and minima from the profiles.

Table 4.5 Effect of varying model parameters in EGSnrc simulations on mean percentage differences of dose at the maxima and minima, as well as average DTA (left and right) values at penumbra regions from Monaco calculations against EGSnrc simulations. Changes were made to increase the in-line beam width to $2 \mathrm{~mm}$ as well as including tongue $\&$ groove to the MLC model. Uncertainties are statistical uncertainties associated with dose values at different maxima and minima from the profiles.

Table 5.1 Range of motion in 3D for the lung phantom measured by RADPOS detectors at the center of the tumor as well as top and bottom plug surfaces. Values represent the average P-P amplitude of 10 independent measurements along with their standard deviation. Motion in S-I direction as measured from 4DCT images of the EOE and EOI is also presented. 82

Table 5.2 The 95\% confidence interval for the range of motion presented in Table 5.1. 82

Table 5.3 The $95 \%$ confidence intervals of the measured sinusoidal motion for different $\mathrm{P}$ $\mathrm{P}$ amplitudes in A-P, L-R and S-I.

Table 5.4 Inter- and intra-day amplitude variations in S-I, A-P and L-R directions for tumor, top and bottom RADPOS detectors for sinusoidal motion profiles. 
Table 5.5 Reproducibility of the average P-P amplitude and amplitude variations in S-I, A$\mathrm{P}$ and L-R directions for tumor, top and bottom RADPOS detectors for irregular motion profiles. Values represent the standard deviation of the average P-P amplitude and average value of amplitude variations across individual motion traces, respectively.

Table 5.6 Inter-day amplitude variations in S-I, A-P and L-R directions for tumor, top and bottom RADPOS detectors for irregular motion profiles. ......................................... 90

Table 6.1 Summary of various sources of dosimetric uncertainty for film measurements. 98

Table 6.2 Summary of various sources of dosimetric uncertainty for RADPOS

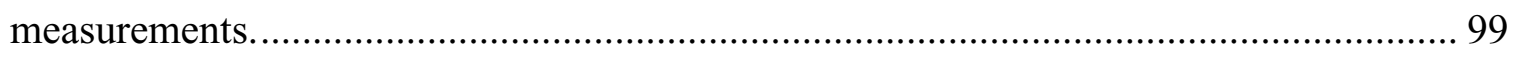

Table 6.3 Mean registration errors with their standard deviations in A-P, L-R, S-I and 3D.

Table 6.4 Passing rates of $1 \mathrm{D}$ and $2 \mathrm{D}$ gamma comparisons of $2 \% / 2 \mathrm{~mm}$ criterion for MC simulations against film measurements on the stationary Quasar phantom. ................. 120

Table 6.5 Dose values at the center of tumor from measurements with film and RADPOS as well as MC simulations on the stationary Quasar phantom.

Table 6.6 Dose values at the center of tumor from measurements with film and RADPOS as well as MC simulations on the stationary deformable phantom.

Table 6.7 Dose values at the top and bottom surfaces of the plug from MC simulations and RADPOS measurements on the stationary deformable phantom. The top surface RADPOS was placed at an offset position of $1 \mathrm{~cm}$ from the tumor center. 123

Table 6.8 Passing rates of $1 \mathrm{D}$ and 2D gamma comparisons of $2 \% / 2 \mathrm{~mm}$ criterion for $\mathrm{MC}$ simulations against film measurements on the breathing Quasar phantom. 127

Table 6.9 Dose values at the center of tumor from measurements with film and RADPOS as well as MC simulations on the breathing Quasar phantom. 127

Table 6.10 Passing rates of $1 \mathrm{D}$ gamma comparisons of $2 \% / 2 \mathrm{~mm}$ criterion for $\mathrm{MC}$ simulations against film measurements on the breathing deformable phantom for sinusoidal, typical, highly irregular and large hysteresis between the tumor and diaphragm motion respiratory motion profiles. First column of gamma passing rates corresponds to the dose profiles shown in Figures 6.16-6.19. The average values from all irradiation sets are shown in the second column. 133 
Table 6.11 Dose values at the center of tumor from measurements with film and RADPOS as well as MC simulations on the breathing deformable phantom during sinusoidal, typical, highly irregular and large hysteresis between the tumor and diaphragm motion respiratory motion profiles.

Table 6.12 Dose values at the bottom surface of the plug from MC simulations and RADPOS measurements on the breathing deformable phantom during sinusoidal, typical, highly irregular and large hysteresis between the tumor and diaphragm motion respiratory motion profiles.

Table 6.13 Dose values at the top surface of the plug from MC simulations and RADPOS measurements on the breathing deformable phantom during sinusoidal, typical, highly irregular and large hysteresis between the tumor and diaphragm motion respiratory motion profiles. This RADPOS was placed at an offset position of $1 \mathrm{~cm}$ from the tumor center. 


\section{Chapter 1}

\section{Introduction}

\subsection{Radiation therapy and cancer treatment}

The predicted new cases of cancer in 2017 in Canada were over 206,000 with almost a third dying of the disease ${ }^{1}$. Lung cancer was reported to be the leading cause of death among all other cancer types ${ }^{1}$.

For treatment of cancer, radiation therapy remains an important modality with approximately $50 \%$ of all cancer patients receiving radiotherapy during their course of treatment ${ }^{2}$. The ultimate goal of radiation therapy is to destroy cancer cells by delivering a sufficiently high dose of ionizing radiation to a target volume while minimizing the damage to healthy tissues and organs at risk (OAR). The radiation dose, or dose absorbed by a medium, is defined as the energy deposited by charged particles per unit mass of the medium and has units of $\mathrm{J} / \mathrm{kg}$ also known as Gray (Gy).

External beam radiation therapy (EBRT) uses ionizing radiation generated by a medical linear accelerator (linac) to deliver a prescribed dose to a delineated target volume. A linac is an accelerator that makes use of microwave technology to accelerate electrons in a waveguide. The accelerated electron beam is then made to collide with an X-ray target to produce bremsstrahlung photons. A typical high energy linac provides nominal accelerating potentials of 6 and $18 \mathrm{MV}$, which define the maximum energy of the photon beam. A rotatable beam collimation system that is comprised of the multi-leaf collimator (MLC) and a pair of jaws is then used to shape the generated photon beam. The MLC consists of pairs of opposing leaves that can move in a step-and-shoot or continuous dynamic mode. The beam generation, shaping and collimation systems are located in the 
treatment head which is mounted on a gantry that can rotate around the treatment couch and direct the beam to the target volume in different configurations. The treatment couch can move laterally, vertically and longitudinally. It can also rotate which helps with patient positioning before starting the treatment. The point where the rotation axes of collimator, gantry and couch intersect is the radiation isocenter ${ }^{3}$. Figure 1.1 shows an Elekta Infinity linac with Agility MLC.

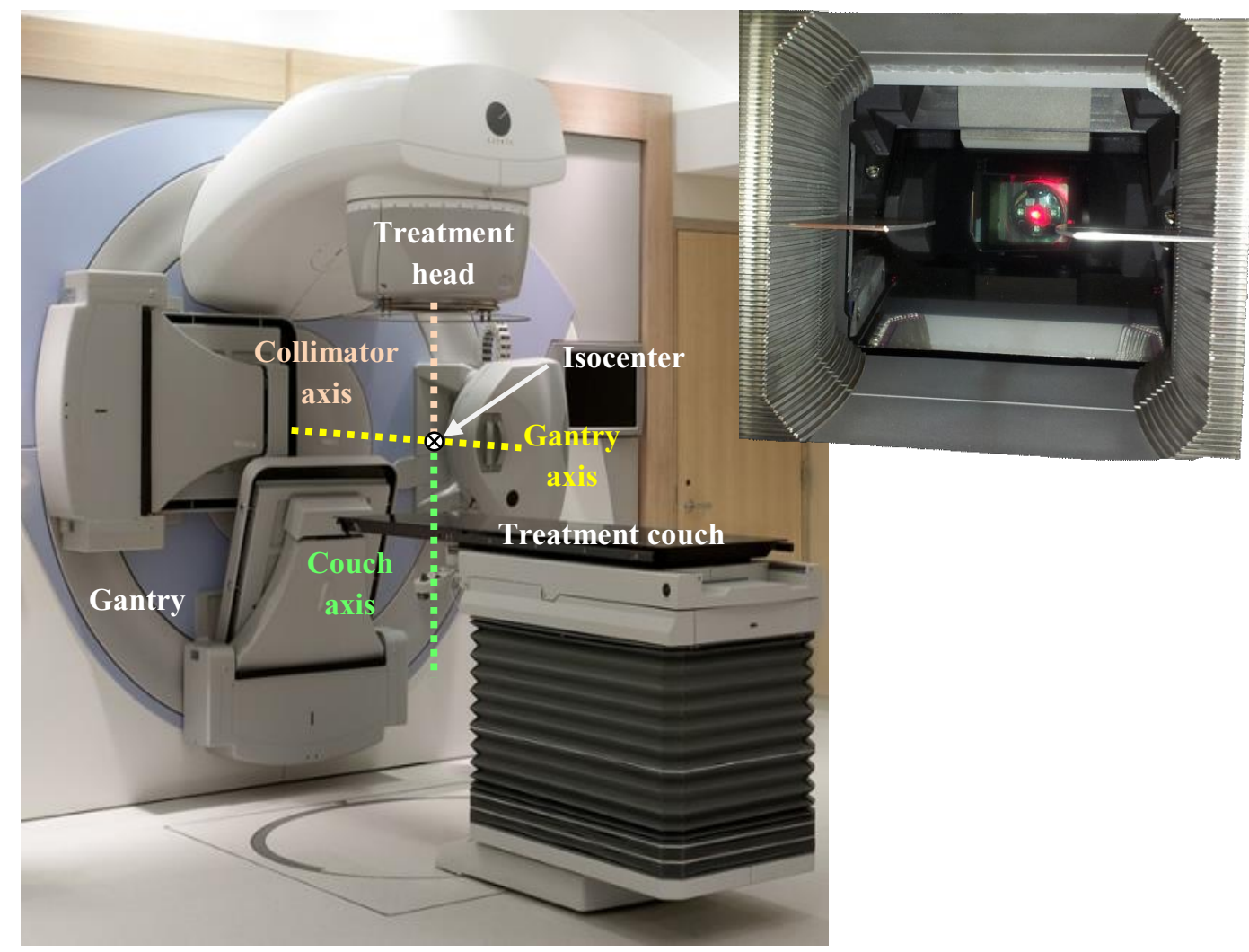

Figure 1.1 A conventional linac - Elekta Infinity with typical components including gantry, treatment head and treatment couch (left) with Agility MLC (right).

The CyberKnife (Accuray Inc., Sunnyvale, CA, USA) is a robotic radiosurgery system that consists of a $6 \mathrm{MV}$ linac mounted on a robotic arm with six degrees of freedom of motion. The unit is capable of delivering highly conformal dose distributions while being guided in real-time to track the target volume. The imaging system responsible for 
real-time tracking consists of two ceiling-mounted X-ray sources as well as the stereoscopic camera. The X-ray system tracks the internal fiducials or bony anatomy while the stereoscopic camera tracks the external LED markers ${ }^{4}$.

The radiation therapy process comprises several sequential steps. The first step after the disease diagnosis and initial consultation is image acquisition. Various imaging modalities such as computed tomography $(\mathrm{CT})$ and magnetic resonance imaging (MRI) can be utilized in this step ${ }^{5,6}$. The next step is called simulation. The goal of the simulation is to establish the patient coordinate system, verification of the treatment field geometry as well as determination of the size, location and extent of the tumor (i.e. target volume) $)^{5-7}$. Once the tumor is localized, the gross tumor volume (GTV), clinical target volume (CTV), internal target volume (ITV) and planning target volume (PTV) are delineated according to Figure 1.2.

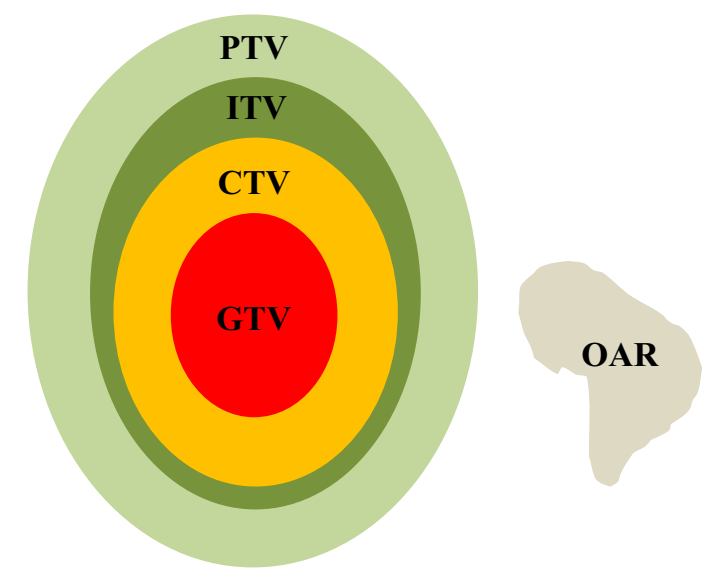

Figure 1.2 Delineations of GTV, CTV, ITV, PTV and OAR used throughout treatment planning process. Adapted from the ICRU reports $50^{7}$ and $62^{8}$.

The GTV is defined as the macroscopic extent of the tumor that is palpable (during examination) or visible (by imaging modalities). The CTV includes the GTV and microscopic extension of disease which has to be eliminated. The ITV consists of the CTV 
plus an internal margin to account for positional variations of the target volume that occur due to organ motion. The PTV includes the ITV and can be described as the ITV plus a setup margin to account for variations in the patient setup for treatment. An organ at risk is defined as normal tissue with a high enough radiation sensitivity to impact the treatment plan, for example requiring changes in beam arrangement or prescribed dose $\mathrm{e}^{7,8}$.

Next, the patient data obtained from CT or other imaging modalities are input to a treatment planning system (TPS) along with the target volume and OAR contours. A treatment plan, which consists of an optimal configuration of radiation beams, is generated and the prescribed dose is calculated with the dose calculation algorithms of the TPS. Finally, the Monitor Units (MU) required to deliver the dose are determined ${ }^{5-7}$. In EBRT, the linac is calibrated such that 1 unit (MU) reading of its monitor chamber corresponds to the dose of $1 \mathrm{cGy}$ in the phantom under the reference conditions. The prescribed dose is generally delivered in several fractions over the course of the treatment.

Cancer can be treated using different radiotherapy techniques. 3D conformal radiation therapy (3D-CRT) uses a combination of static beams that irradiate the tumor from different angles ${ }^{9}$. A more advanced technique is intensity modulated radiation therapy (IMRT) that makes use of non-uniform beam intensities throughout the treatment delivery 9 The beam intensity can be modulated using MLC which may or may not move during delivery of an IMRT treatment plan. Volumetric modulated arc therapy ${ }^{\mathbf{1 0}}$ (VMAT) is a form of IMRT that delivers intensity modulated fields with continuous gantry rotation. An advantage of VMAT is that it increases the efficiency of treatment plan delivery by reducing treatment time ${ }^{\mathbf{1 0}}$. Generally, any treatment plan delivery is divided into several segments known as control points that are delivered sequentially. Each control point 
specifies the state of the machine (e.g. MLC, jaws and gantry positions) as well as the number of MU delivered during that segment. A typical VMAT lung treatment fraction delivery takes approximately 15-20 min which includes patient preparation and setup. The total prescribed dose would be about $60 \mathrm{~Gy}$ which is delivered in 30 daily fractions. The CyberKnife system can be used for lung stereotactic body radiotherapy (SBRT) treatments ${ }^{11-14}$. SBRT is typically used for early-stage lung cancers when surgery is not an option and is characterized by high dose gradient and smaller margins compared to conventional radiotherapy. It is a hypofractionated regimen where total treatment dose of approximately 50-60 Gy is delivered in 3-8 fractions of 20-45 min including setup prior to treatment and depending on the treatment technique (i.e. conformal, VMAT, CyberKnife).

\subsection{Brief review of the lung anatomy}

The human lungs are elastic organs and are divided into five independent compartments called lobes. The left lung consists of the upper and lower lobes while the right lung has three lobes: upper, middle and lower. In each lung lobar surfaces slide against each other to allow shape changes during respiration ${ }^{15,16}$. The lung lobes are shown in Figure 1.3.

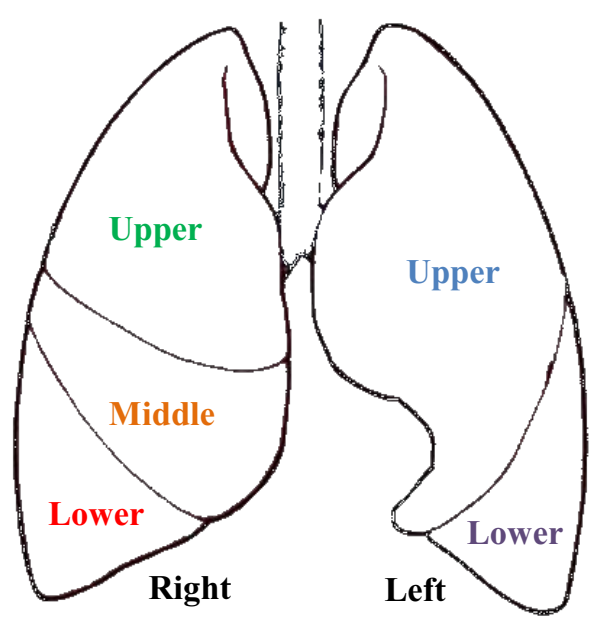

Figure 1.3 Anatomy of lung showing the five lobes on the left and right lung. 
The diaphragm is a thin muscle that separates the thoracic cavity from the abdominal cavity. During inhalation, contraction of the diaphragm increases the volume of the thorax and allows airflow into the lungs. At expiration, the diaphragm relaxes and air is pushed out of the lungs to decrease the thoracic volume. In addition to diaphragm, contractions of the intercostal muscles during respiration to move the rib cage contribute to the deformations of the thorax ${ }^{17,}{ }^{18}$. Because the relative contribution of these mechanisms to respirations is not constant, variations of the breathing motion might be observed within a single breathing cycle (intra-cycle variation) or between breathing cycles (inter-cycle variation). The intra-cycle variation, also referred to as hysteresis, occurs when two different motion paths are followed during inhale and exhale. The inter-cycle variations on the other hand, refers to different motion paths followed during two or more breathing cycles ${ }^{19}$.

\subsection{Impact of patient respiratory motion on radiotherapy treatment delivery}

Accuracy of radiotherapy is limited by the fact that the dose distribution from the treatment plan is based on a single state of the patient anatomy at the time of dose calculation. However, the patient's anatomy and position often vary between treatment fractions (inter-fraction motion) or during one fraction (intra-fraction motion) $)^{20-22}$. As a result of these positional uncertainties, a significant deviation might be observed between the dose prescribed and received by a target volume ${ }^{22}$.

Inter-fraction motion is associated with changes in the position of the CTV from one treatment fraction to another. The reason for this change could be shrinkage of the tumor or changes in the patient's condition such as weight gain/loss. Daily 
localization/alignment of the CTV prior to each treatment fraction using on-board imaging equipment can help to minimize this problem ${ }^{22-24}$.

It has been shown that respiratory motion is an intra-fraction motion that contributes largely to the geometric uncertainties in thoracic and abdominal sites ${ }^{21,22,25}$. The magnitude of the motion of a lung tumor due to respiratory motion varies with the position of the tumor within the lung. The principal component of this motion is in the superior-inferior (S-I) direction and can be as large as $20 \mathrm{~mm}$ peak-to-peak (P-P). In the anterior-posterior (A-P) and left-right (L-R) directions it does not exceed $10 \mathrm{~mm}$ P-P21, 26, 27. These anatomical directions are defined in Figure 1.4.

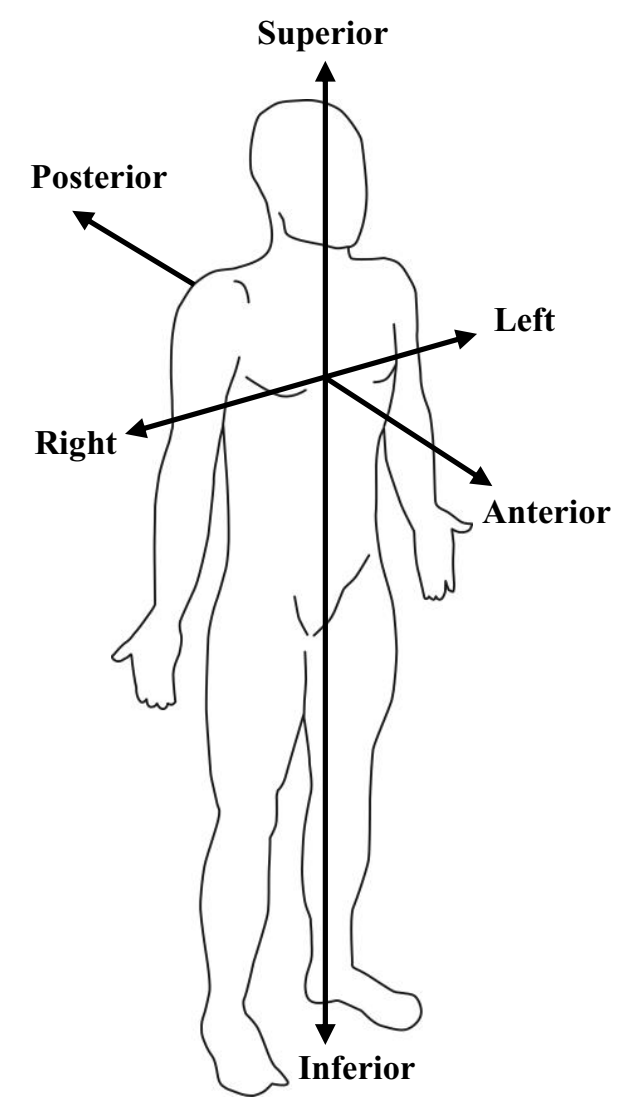

Figure 1.4 Anatomical directions 
The main impact of respiratory motion is the blurring of the dose distribution along the path of the motion. Such blurring reduces the sharpness of dose distribution and increases the beam penumbra as shown in Figure 1.5. The amount of blurring is independent of the treatment technique and depends on the properties of the respiratory $\operatorname{motion}^{10,11}$.

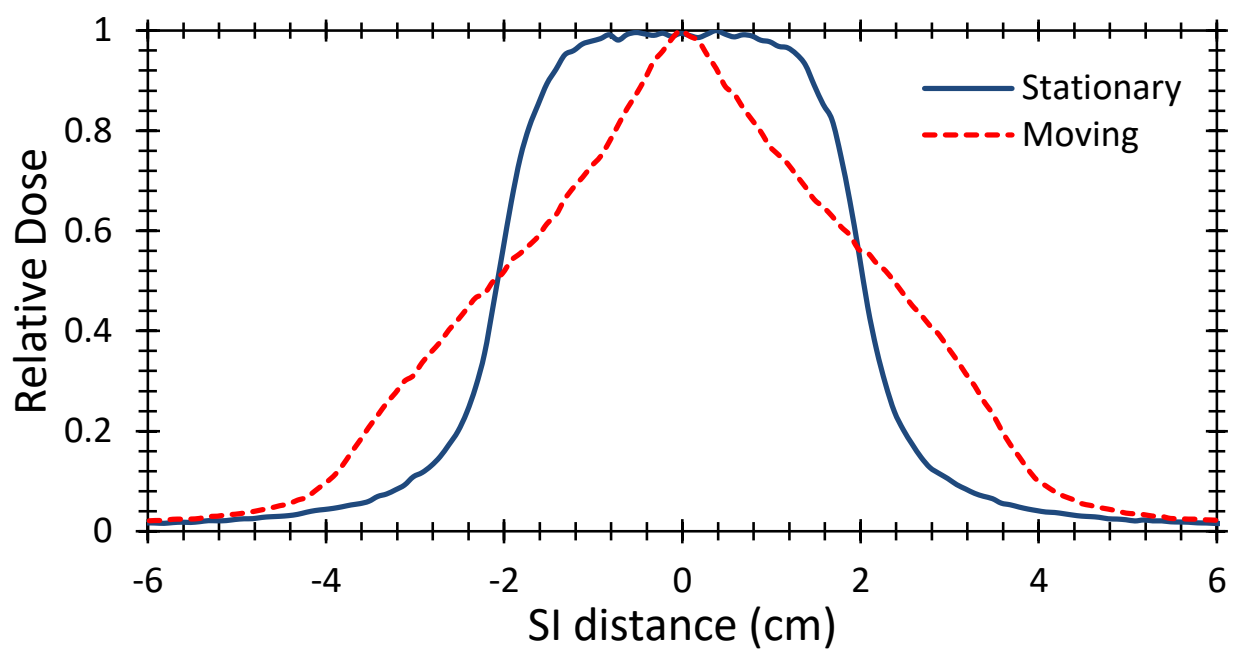

Figure 1.5 Dose profiles for stationary (solid blue) and moving/breathing (dashed red) states of the anatomy. Respiratory motion results in a broader penumbra and blurs dose distribution along the motion path.

Localized deformation (Figure 1.6) in the dose distribution is another effect that occurs due to the motion and deformation of the internal anatomy from one respiratory phase to another (e.g. exhale to inhale). Such deformations can result in up to $5 \%$ error in the delivered dose compared to the planned dose in the localized dose values ${ }^{20,28}$.

When dynamic beam delivery techniques are used, an additional effect caused by the interplay between motion of the beam collimators and target motion is observed, inducing further deviation of $1 \%-10 \%$ between the delivered and prescribed doses ${ }^{20,21,29-}$ 36. This effect is known as the interplay effect and is shown in Figure 1.7. 


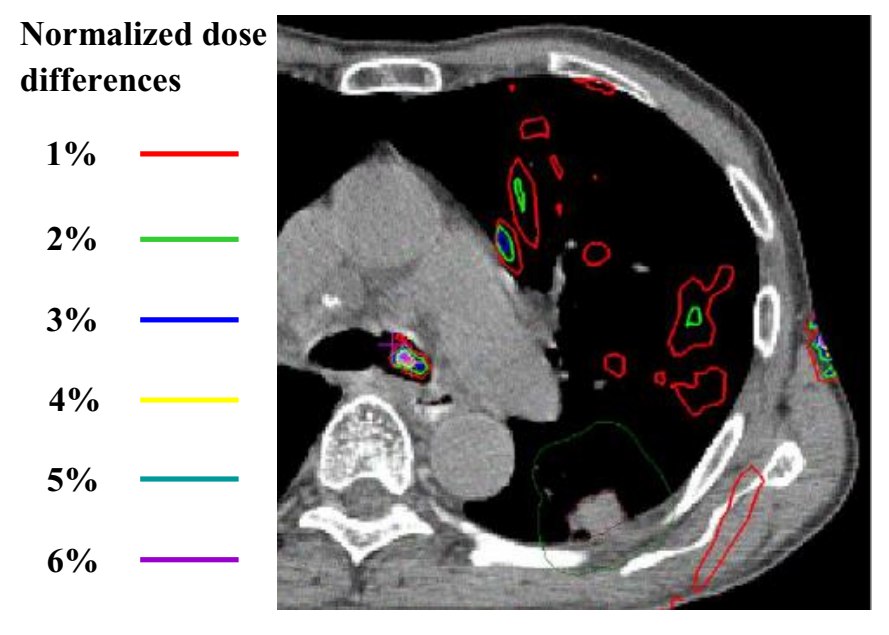

(a)

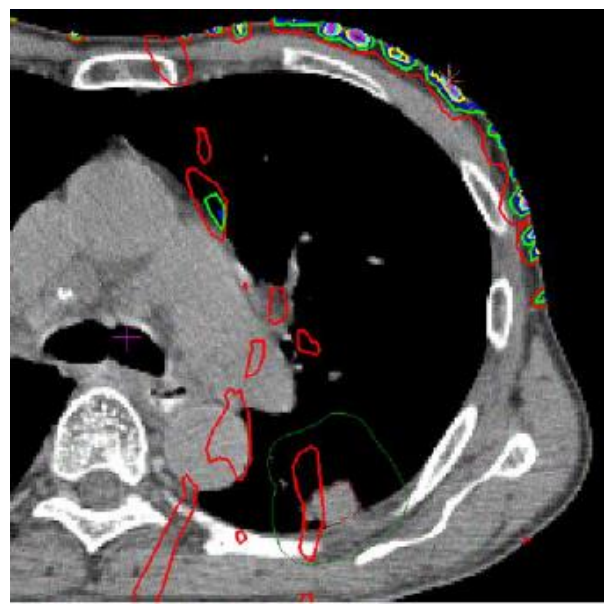

(b)

Figure 1.6 Subtracted doses for dose distributions calculated in exhale and inhale respiratory phases with the same treatment plan. Without the localized dose deformations, the differences shown in (a) inhale-exhale and (b) exhale-inhale should be zero. Dose differences are normalized to the calculated dose in inhale. Adapted with permission from Bortfeld et al., 2004 ${ }^{20}$.
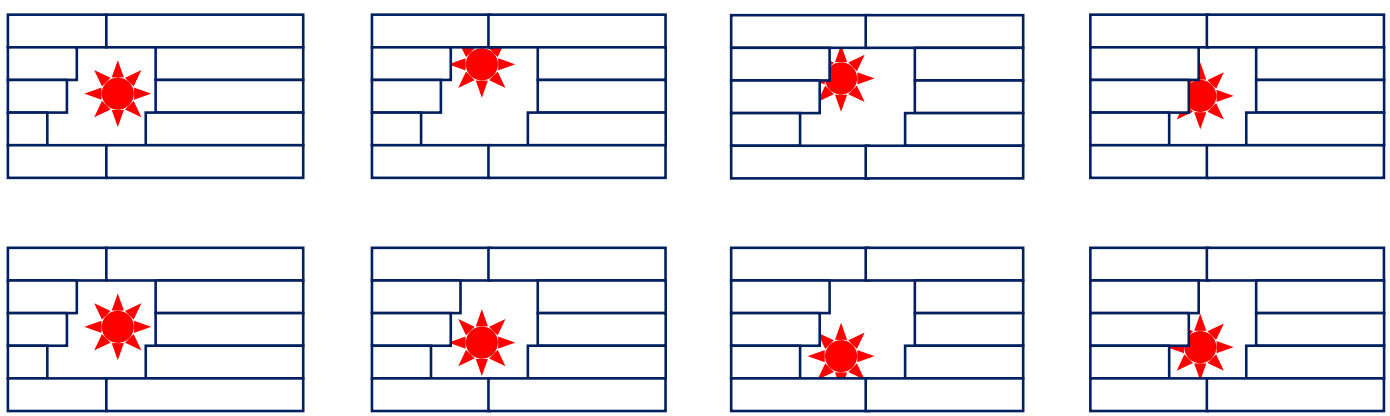

Figure 1.7 Interplay effect between the target volume (red star) and multi-leaf collimator. The target moves superior-inferior while leaves sweep from left to right. The dose received by the target volume is a function of the time it is covered by the leaves and thus depends on the relation between motion of the target and motion of the leaves. The target is receiving a higher dose in the second delivery (bottom) than in the first (top).

\subsection{Management of respiratory motion in radiotherapy}

Several approaches can be used to reduce the impact of respiratory induced tumor motion in radiotherapy $y^{21}$. Regardless of the chosen approach, a common practice is to add a safety margin (i.e. ITV) according to the magnitude of respiratory motion around the target volume (i.e. CTV) during treatment planning ${ }^{7,8,21}$. The main drawback of large 
margins, despite ensuring that tumor receives the prescribed dose, is the over-dosage of healthy tissues around the tumor ${ }^{7,8,22}$. The margin can be reduced, however, if the right techniques are used to decrease the magnitude of motion. Voluntary/coached ${ }^{37-40}$ and forced breath-hold ${ }^{41,42}$ techniques significantly reduce the respiratory induced tumor motion and position critical organs (e.g. heart) away from the treatment beam due to the expansion of the thorax. Forced shallow breathing (FSB) is another technique that applies a pressure against the abdomen which reduces the diaphragm motion and as a result motion of the tumor ${ }^{43-45}$. Another method, known as respiratory gating, involves activating the radiation beam whenever the respiratory signal is within a pre-set position or phase window. Gated radiotherapy results in longer treatments times due to the pauses in the beam delivery ${ }^{40,46-48}$. A more complex technique is real-time tracking of the tumor motion ${ }^{49-52}$ to identify its position and adjust the position of the beam based on the delays in the beam positioning system. As a result of continuous beam delivery, treatment times will be shorter compared to gating approaches ${ }^{53}$.

\subsection{Incorporating respiratory motion in dose calculation}

The effects of respiratory motion mentioned earlier can cause a deviation between the planned and delivered doses. Thus, it is important to account for these effects in the calculation of dose distribution. One early approach that analytically incorporates the impact of respiratory motion in the calculated dose is convolution of the static dose distribution with a probability distribution function (PDF) that describes the characteristics of the respiratory motion ${ }^{54-56}$. Convolution-based methods sufficiently model the blurring effect of the respiratory motion. One limitation of these methods is the assumption that the dose distribution is shift invariant which results in inaccuracies at interfaces of different 
density tissues ${ }^{46,47}$. In addition, with convolution-based approaches patient motion can only be modeled as rigid body translations and deformations in the anatomy are not considered ${ }^{20}$, 57.

A more accurate approach that accounts for both translations and deformations in the anatomy is accumulation of the dose distributions calculated on multiple respiratory phases. Dose distributions calculated on each respiratory phase are mapped back to a reference (planning) phase and then combined, using a weighted sum (e.g. time spent in each phase), to acquire the cumulative dose distribution. A deformable image registration (DIR) algorithm is used to generate the anatomy mapping required from each respiratory phase to the reference phase ${ }^{59,60}$. This concept is incorporated in four-dimensional (4D) dose calculation algorithms and is shown in Figure 1.8.

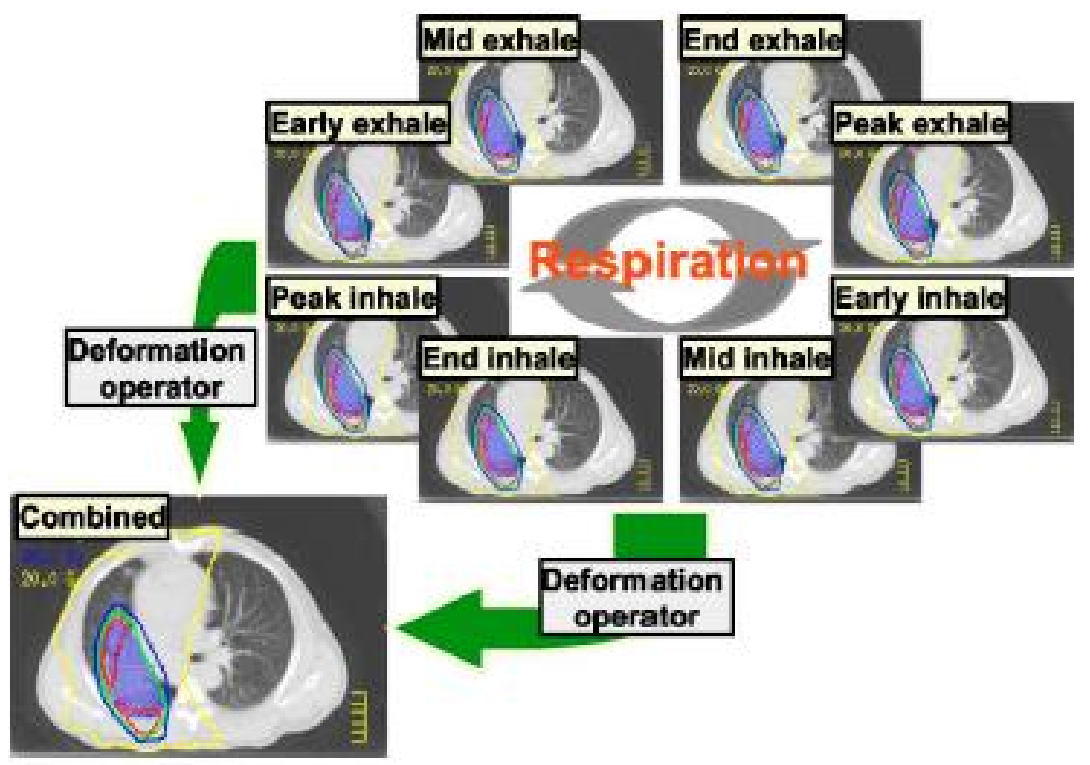

Figure 1.8 The process of 4D dose calculation. Dose distribution is calculated on multiple respiratory phases and then mapped back to a reference phase to construct the cumulative dose distribution. Obtained with permission from Keall et al., $\mathbf{2 0 0 5}^{61}$. 
Such 4D dose calculations require 4DCT images that allow quantification of organ motion as a function of respiratory phases. A 4DCT scan is obtained by acquiring image slices while simultaneously recording respiratory signals and finally tagging each image slice with the corresponding respiratory phase ${ }^{62-64}$. Image acquisition might be performed in either cine (repeated image acquisition at each axial position) ${ }^{65,66}$ or helical (with small pitches) ${ }^{63,65}$ modes. To obtain respiratory signals different approaches including abdominal belt $^{67}$ or external markers ${ }^{63,64}$ might be used. The information obtained from a 4DCT scan is useful in understanding the motion trajectory of the tumor and generate deformation maps used in $4 \mathrm{D}$ dose calculation algorithms ${ }^{60,68}$.

\subsection{Thesis outline and motivation}

This thesis covers the work done to experimentally validate a novel 4D Monte Carlo simulation tool for reconstructing the delivered dose accounting for respiratory motion. This is an important step before a dose calculation tool can be used for treatment verification in the clinic. Also covered in this thesis is the Monte Carlo modeling of an Elekta Infinity linac with Agility MLC as well as investigating the differences observed between the measured and Monaco calculated doses for a small radiation field.

Chapter 2 reviews physical and dosimetric principles of all detectors that were used for dose measurements presented in the subsequent chapters. Ionization chamber, diode, Gafchromic EBT3 film and RADPOS 4D dosimetry system (dose and position measurement) are dosimeters that are presented in this chapter. Chapter 3 describes the Monte Carlo dose calculation user codes and methodology used for studies in this work. It includes description of the BEAMnrc, DOSXYZnrc and 4DdefDOSXYZnrc user codes. Chapter 4 covers details on Monte Carlo modeling of the Elekta Infinity linac that was 
used for experimental studies in this work. Additionally, a virtual source model was developed and sensitivity of different model parameters on the performance of the model to calculate the isocentric photon fluence was studied. Finally, application of both models to explain the dose discrepancies obtained from Monaco calculations and film measurements for a small field are presented in this chapter. Chapter $\mathbf{5}$ introduces the respiratory motion phantoms that were used, in conjunction with film and RADPOS, to verify 4D Monte Carlo dose calculations. Details on motion and structure characteristics of rigidly moving (Quasar respiratory motion) and deformable programmable (in-house design and development) lung phantoms are included. Chapter 6 focuses on experimental verifications using the phantoms presented in the previous chapter. Film and RADPOS were used to verify delivered dose during static and VMAT treatment deliveries. The RADPOS system was also used to measure the phantom motion during treatment delivery. Initial verifications were performed on the Quasar phantom where a rigid translation of sinusoidal breathing motion was simulated. Similar sinusoidal motion was simulated on the deformable phantom to validate the performance of the 4DdefDOXYZnrc user code in a deforming anatomy. Finally, more irregular and realistic breathing motions were tested to complete phantom studies. Chapter 7 is a brief summary of the major conclusions of each Chapter, with remarks on outlook and potential future work. 


\section{Chapter 2}

\section{Dose measurements}

\subsection{Cylindrical ionization chamber}

Cylindrical ionization chambers are the most popular type of ionization chambers that are used for dose measurements in radiotherapy ${ }^{3}$. In principle, they are cavities filled with gas (usually air) and a central electrode to collect the charge produced by ionizing radiation. They consist of a conducting wall separated from the central electrode with an insulator to minimize the leakage current when the polarizing voltage is applied to the chamber. To further reduce the leakage, guard electrodes are also implemented within the chamber. Ionization chambers are reusable, accurate and precise dosimeters and in conjunction with an electrometer they can be used as on-line readout dosimeters to measure the dose during and post irradiation ${ }^{3}$. The electrometer also provides a high voltage external bias to create an electric field in the chamber. A schematic of a cylindrical ion chamber connected to electrometer is shown in Figure 2.1.

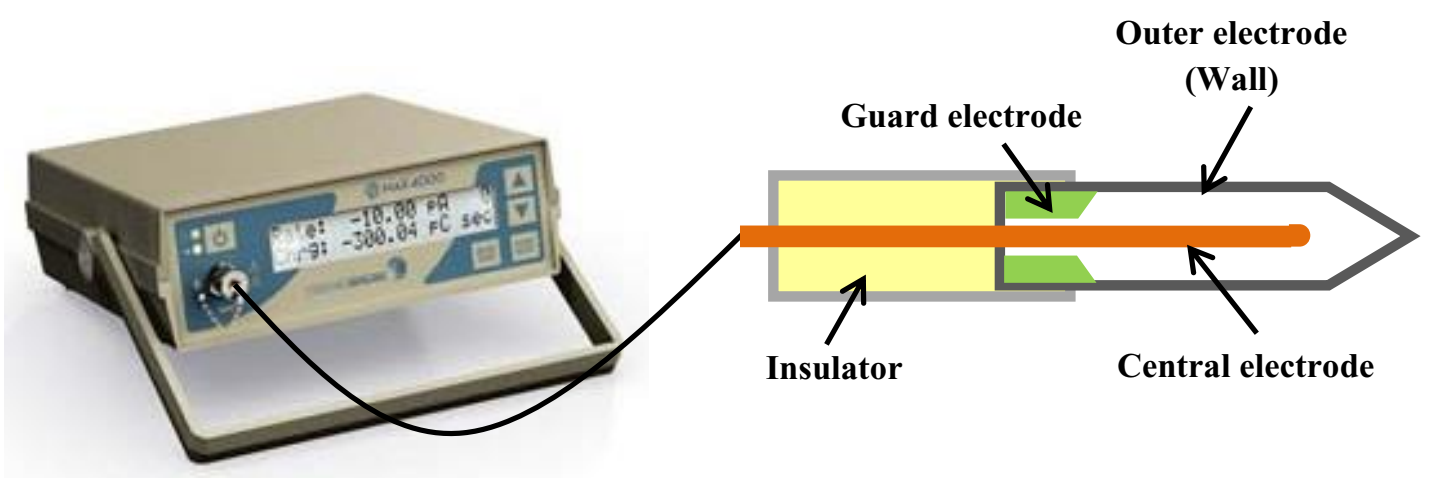

Figure 2.1 Schematic of a cylindrical ionization chamber (right) connected to an electrometer (left). 
The ionization charge Q generated in the cavity is related to the absorbed dose in air $\left(\mathrm{D}_{\text {air }}\right)$ through following formulation:

$$
D_{\text {air }}=\frac{Q}{m_{\text {air }}}\left[\frac{W_{\text {air }}}{e}\right]
$$

where $\left(\frac{W_{\text {air }}}{e}\right)$ is the average energy required to produce one ion pair in air at a reference temperature and pressure of $20^{\circ} \mathrm{C}$ and $101.325 \mathrm{kPa}$, respectively, and is equal to $33.97 \mathrm{eV} /$ ion pair. Because the chamber cavity is vented, the ionization chamber reading needs to be corrected ( $\mathrm{P}_{\mathrm{T}, \mathrm{P}}$ correction factor) to account for the change in the mass of air in the chamber cavity due to changes of the ambient temperature and pressure from the reference condition ${ }^{3}$.

The chamber reading is converted to absorbed dose to water using the AAPM Task Group 51 absolute dose calibration $\operatorname{protocol}^{69}$ :

$$
D_{w}=M_{Q} N_{D, w}^{60} k_{Q}
$$

In this equation $\mathrm{M}_{\mathrm{Q}}$ is the corrected reading from chamber and $\mathrm{N}_{\mathrm{D} \text {, }}(\mathrm{Gy} / \mathrm{nC})$ is the chamber-specific calibration coefficient in terms of the absorbed dose to water obtained from the standards laboratory. Finally, $\mathrm{k}_{\mathrm{Q}}$ is the beam quality correction factor to convert the calibration coefficient for a ${ }^{60} \mathrm{Co}$ beam to the one for beam quality $\mathrm{Q}$, determined during clinical reference dosimetry.

\subsection{Silicon diode}

Diode dosimetry systems used in radiotherapy are often p-type silicon diodes since they produce a much smaller dark current ${ }^{3}$. A p-type silicon diode dosimeter is formed by counter-doping n-type silicon into a p-type surface that creates a p-n junction diode. At 
equilibrium, the majority carriers from each side, i.e. electrons from $\mathrm{n}$ side and holes from $\mathrm{p}$ side, diffuse to the other side. This diffusion leaves the $\mathrm{n}$ side with un-paired holes and the $\mathrm{p}$ side with un-paired electrons which creates an intrinsic potential difference that does not allow further diffusion of these carriers. Ionizing radiation produces electron-hole pairs in the entire silicon diode including the p-n junction (depletion region). Charge carriers produced in the depletion region are swept across by its intrinsic potential and create a current that is then collected by an electrometer (no need for external bias voltage) ${ }^{3,70}$. The excess minority carriers within approximately one diffusion length from the junction are able to reach the junction (before recombination) and contribute to this current as well. The dark current of the diode exists even without diode being irradiated and it is subtracted from the current measured during irradiation. Like ionization chambers, diodes are on-line readout dosimeters. Figure 2.2 shows schematic of a typical diode dosimeter construction.

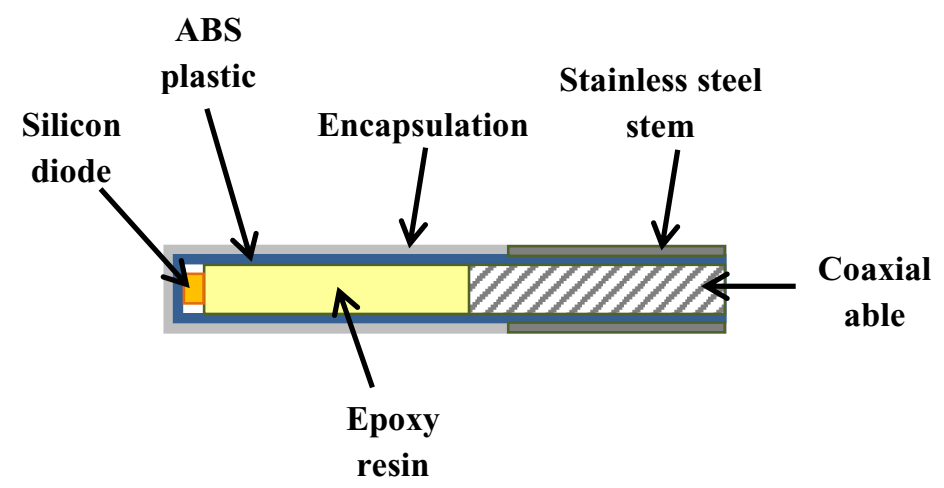

Figure 2.2 Schematic of construction of a silicon photon diode dosimeter. Adapted from Cranmer-Sargison et al. ${ }^{71}$.

The epoxy layer absorbs low energy scattered photons which helps adjust the energy response of the diode. Diodes are highly sensitive and have a small sensitive volume (approximately $10^{-2} \sim 10^{-3} \mathrm{~mm}^{3}$ ) which makes them a very good candidate for dosimetry of small radiation field sizes. The sensitivity of diodes decreases with the accumulated dose 
due to the radiation damage. The dose response of diodes depends on temperature, instantaneous dose rate, and beam energy. Increasing temperature and dose rate result in increasing sensitivity of diodes due to higher generation rate of charge carriers. The energy dependence of diodes is due to the different materials (e.g. electrode attachments, etc.) used for detector construction. Some angular dependency of the diode response to radiation beam can be also observed. This angular dependency is mainly a result of changes in beam transmission through varying thicknesses of the enclosing or cable. When used for absolute dosimetry, diodes are usually cross-calibrated against ionization chambers and the derived calibration coefficient $F_{\text {cal }}(\mathrm{Gy} /$ diode reading) is used to convert diode readings to the absorbed dose $\mathrm{e}^{3,70}$. The calibration coefficient is the ratio of a known dose to diode reading.

\subsection{Gafchromic EBT3 film}

Films are 2D dose detectors that provide a high spatial resolution for dosimetry purposes in radiotherapy. This makes films a powerful tool to verify the dose distribution delivered by a patient treatment plan such as IMRT and VMAT or to be used for general quality assurance of linacs ${ }^{72,73}$.

Gafchromic EBT films are a type of radiochromic films which were designed to replace radiographic (silver halide) films ${ }^{72,73}$. The most recent type of Gafchromic EBT films are EBT3 (Ashland, Wayne, NJ, USA) films. The structure of the EBT3 film is shown in Figure 2.3. The film consists of a $28 \mu \mathrm{m}$ active layer $\left(Z_{\mathrm{eff}}=7.26\right)$ which contains an active component, a marker dye, stabilizers and other components. This layer is sandwiched between two $125 \mu \mathrm{m}$ layers of matte polyester $\left(Z_{\mathrm{eff}}=6.64\right)$. These values are comparable with $Z_{\text {eff }}$ of water which is equal to 7.42 . 
Matt polyester, $125 \mu \mathrm{m}\left(Z_{\text {eff }}=6.64\right)$

Active layer, $28 \mu \mathrm{m}\left(Z_{\text {eff }}=7.26\right)$

Matt polyester, $125 \mu \mathrm{m}\left(\mathrm{Z}_{\mathrm{eff}}=6.64\right)$

Figure 2.3 Structure of Gafchromic EBT3 film

Unlike radiographic films, Gafchromic films are self-developing and do not require any kind of chemical processing post irradiation. They undergo a color change when exposed to ionizing irradiation. The change of the color is due to the polymerization of the colorless monomers of the film's active layer. Gafchromic films are nearly tissue equivalent, have low energy dependence (for energies above $100 \mathrm{keV}$ ) and are relatively insensitive to visible light $t^{3,72,73}$.

Overall, the darkness of the film, i.e. change in its optical density (OD), is proportional to the absorbed dose. The net optical density is determined from the change in the optical density from pre- to post-irradiation and is calculated according to the following formulation ${ }^{73}$ :

$$
n e t O D=O D_{i r r}-O D_{\text {blank }}=\log _{10}\left(\frac{I_{\text {blank }}}{I_{\text {irr }}}\right)
$$

where $\mathrm{I}_{\mathrm{blank}}$ and $\mathrm{I}_{\mathrm{irr}}$ are unirradiated and irradiated intensities (transmission through the film), respectively, which are measured using a commercial flatbed scanner such as an EPSON 10000XL (Suwa, Naogna, Japan). A calibration curve is required to convert the netOD to the absorbed dose. The calibration curve is a plot of known dose values (measured by a calibrated ionization chamber) vs. netOD. This curve is usually a fit with a polynomial function with order $\mathrm{n}$ as shown in the following equation: 


$$
\text { Dose }=\text { a.netOD }+ \text { b.netOD }{ }^{n}
$$

Parameters $\mathrm{a}, \mathrm{b}$ and $\mathrm{n}$ are determined during the fitting procedure. A typical calibration curve for EBT3 film is shown in Figure 2.4.

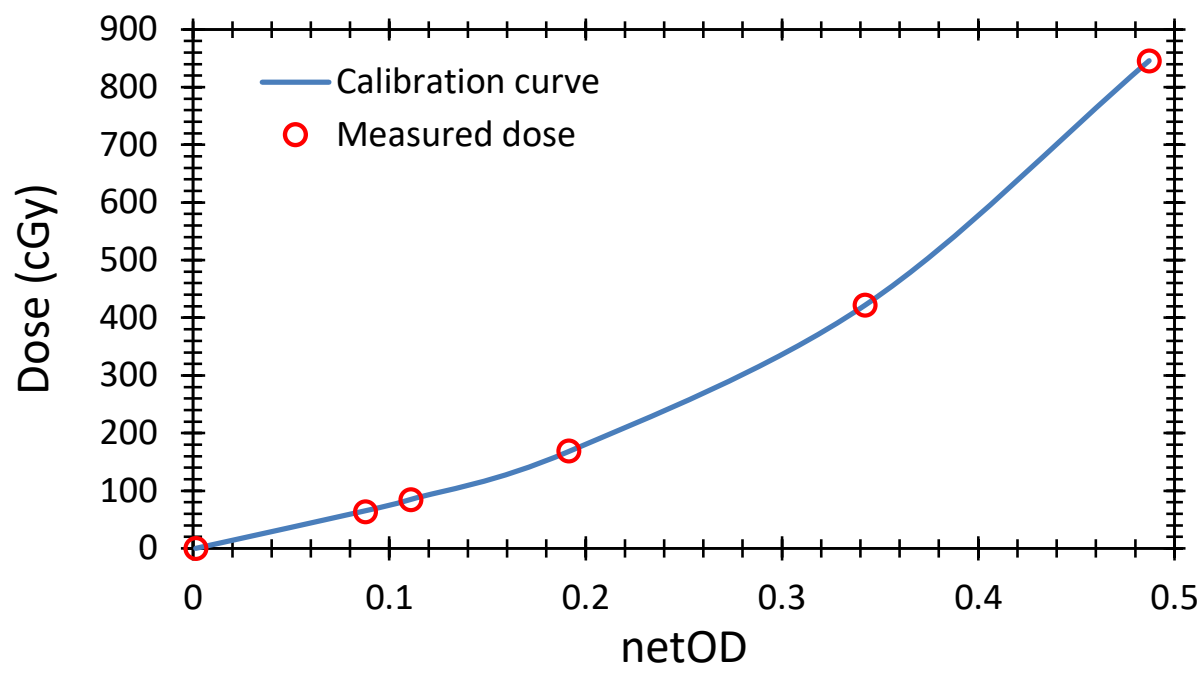

Figure 2.4 Calibration curve for EBT3 film to convert change in the optical density to absorbed dose. The maximum irradiated calibration dose is $845.7 \mathrm{cGy}$.

\subsection{RADPOS}

RADPOS is a 4 D dosimetry system ${ }^{74}$ (Figure 2.5 ) that consists of a probe that combines a microMOSFET dosimeter and an electromagnetic positioning sensor. The MOSFET reader in combination with the microMOSFET performs dose measurements. The 3D-Guidance tracker, pre-amplifier, transmitter and positioning sensor are responsible for position tracking. RADPOS is capable of simultaneous and real-time monitoring of dose and position. The microMOSFET and position sensors are separated by $8 \mathrm{~mm}$ to limit perturbation of the incident radiation beam near the dosimeter ${ }^{74}$. A schematic of the RADPOS probe is shown in Figure 2.6. 


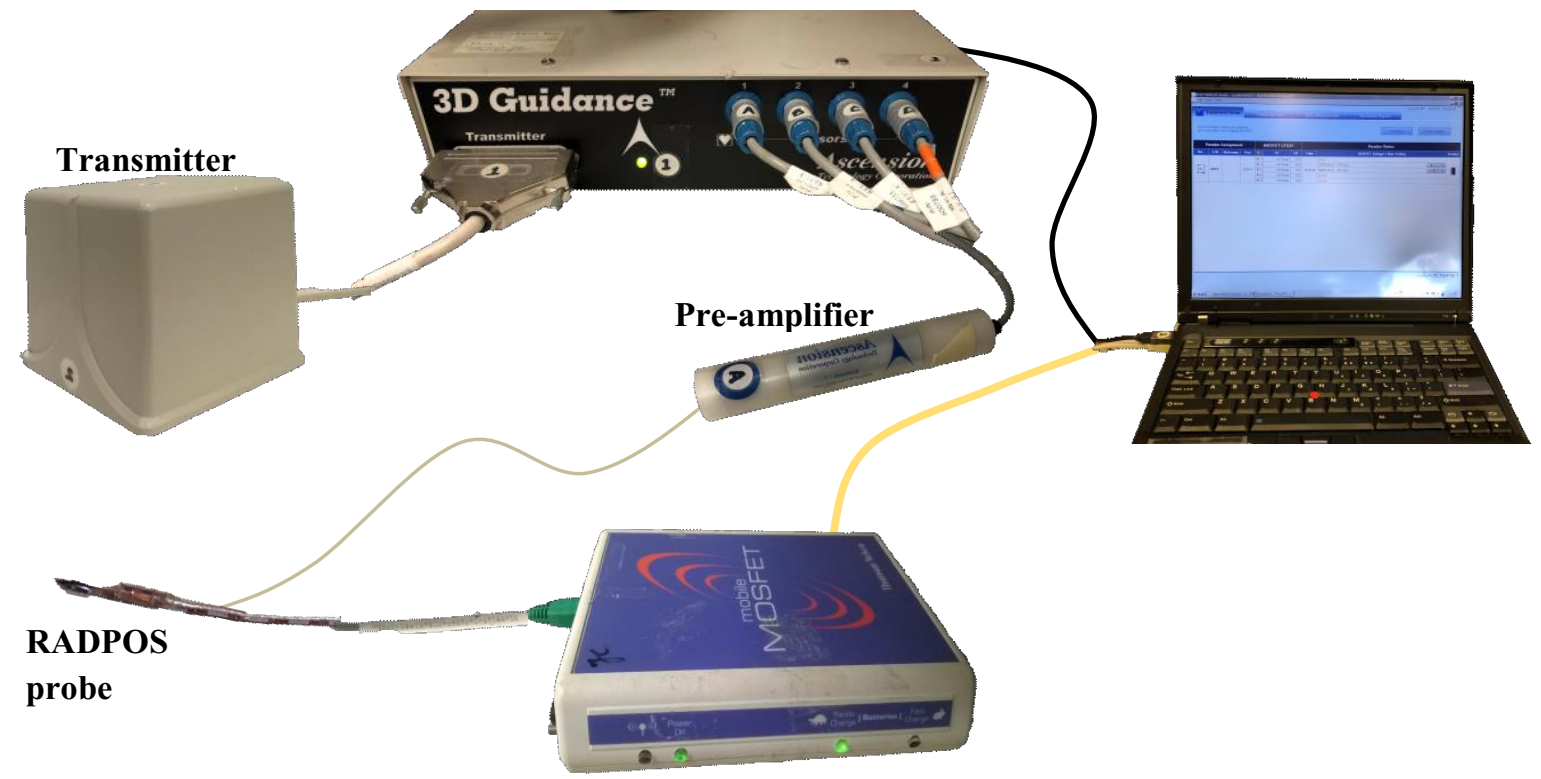

Figure 2.5 An overview of the RADPOS 4D dosimetry system with its components including MOSFET reader, 3D-Guidance tracker, pre-amplifier, transmitter and probe. MOSFET reader and 3D-Guidance tracker are connected to a host PC that runs the RADPOS software.

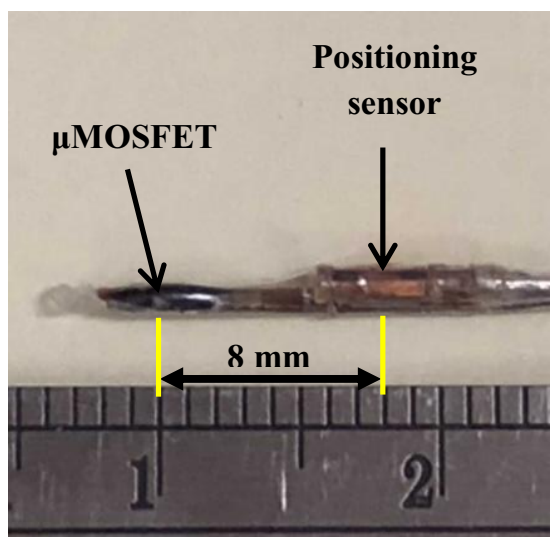

Figure 2.6 The RADPOS probe. The microMOSFET and position sensors are separated by $8 \mathrm{~mm}$. 


\subsubsection{Dose measurement}

The dosimeter used in the RADPOS dosimetry system is the metal-oxide semiconductor field effect transistor (MOSFET). Similar to diodes, a p-type MOSFET is built by embedding it onto a layer of n-type silicon. The source and drain terminals of the MOSFET lie on top of the p-type silicon layer and the gate terminal is built on top of a silicon-dioxide layer to be insulated from the p-type silicon layer. A schematic of the MOSFET structure is shown in Figure 2.7.

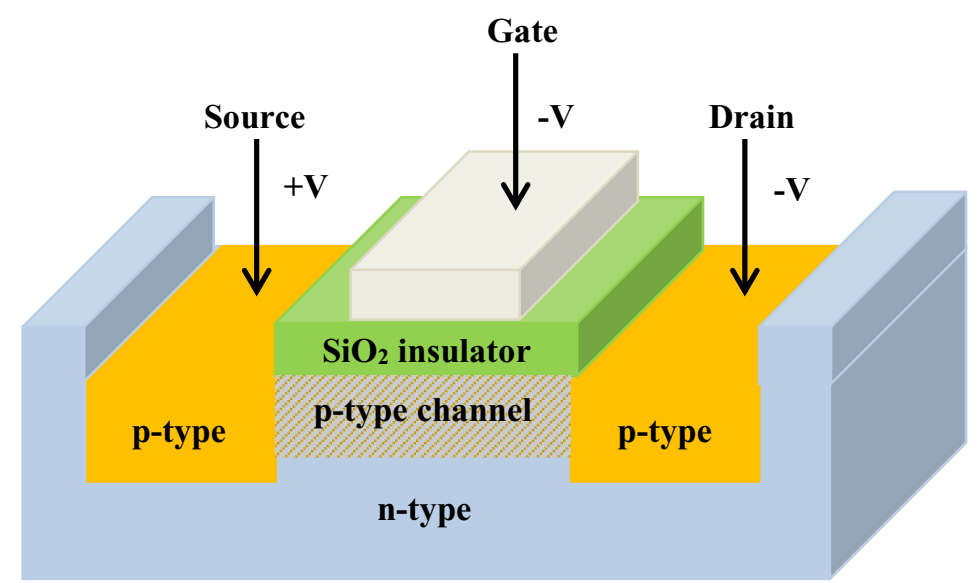

Figure 2.7 Schematic of the structure of a p-type MOSFET (in the read-out mode) used in dosimetry. Adapted from Soubra et al., $1994^{75}$.

When a negative voltage is applied to the gate (i.e. $\mathrm{V}_{\mathrm{g}}<0$ ) it provides an electric field that repels electrons from the n-type substrate of the MOSFET and leaves the area right below the $\mathrm{SiO}_{2}$ insulator with positive carriers (i.e. holes). The negative voltage also attracts holes from the p-type layers of source and drain which make the area even more positive. This is how the p-type channel (rich in positive charge) is formed. The channel is controlled by the gate voltage. At a threshold voltage $\left(\mathrm{V}_{\text {th }}\right)$ and when the appropriate voltage (as shown in Figure 2.7) is applied between drain and source, a current will flow between the source and drain ${ }^{75}$. 
When irradiated, ionizing radiation generates electron-hole pairs inside the $\mathrm{SiO}_{2}$ layer of the MOSFET. Some of these holes will recombine with electrons but some of them will be permanently trapped and create a potential barrier that leads to a higher voltage on the gate needed to induce the conduction channel. This change in the threshold voltage $\left(\Delta \mathrm{V}_{\mathrm{th}}\right)$ before and after irradiation is proportional to the absorbed dose. Accumulation of trapped holes in the MOSFET reduces the sensitivity of the dosimeter and gives it a limited lifespan. Similar to diodes, the MOSFET response depends on energy ${ }^{3,75}$. Although the MOSFET response is in principle temperature-dependent, the dual-MOSFET-dual-bias MOSFET used in this study eliminates this effect ${ }^{75}$.

To perform dose measurements with RADPOS, a calibration coefficient (CF) is required to convert $\Delta \mathrm{V}_{\text {th }}$ to the absorbed dose. ${ }^{74}$ This coefficient is obtained by irradiating the MOSFET simultaneously with the ionization chamber under the reference conditions and using equation 2.5 :

$$
C F=\frac{\text { Dose }}{\Delta V_{t h}} \quad\left[\frac{c G y}{m V}\right]
$$

In this equation the delivered dose is measured by a calibrated ionization chamber.

The RADPOS probe consists of either high sensitivity (TN-1002RDM) or standard sensitivity (TN-502-RDM) microMOSFET dosimeters with sensitive volumes of $0.2 \times 0.2 \times 1 \times 10^{-3} \mathrm{~mm}^{3}$ and $0.2 \times 0.2 \times 5 \times 10^{-4} \mathrm{~mm}^{3}$, respectively. The software used for the RADPOS system allows both manual (post-irradiation) and automatic (at user-defined time intervals) logging of the dose. 


\subsubsection{Position measurement}

The positioning sensor of the RADPOS probe is a small cylinder $8 \mathrm{~mm}$ in length and $1.3 \mathrm{~mm}$ in diameter. The RADPOS transmitter generates a pulsed 3D DC magnetic field. The transmitter consists of three coils that are positioned perpendicularly (along the $\mathrm{x}, \mathrm{y}, \mathrm{z}$ directions) and produce sequential trapezoidal magnetic fields (steady-state on/off periods of $2 \mathrm{~ms}$, build-up and fall-off periods of $250 \mu \mathrm{s}$ ) when the DC current is passed through them. This signal is proportional to the inverse cube of the distance $\left(1 / \mathrm{r}^{3}\right)$ from the transmitter and is measured by the position sensor which is a three-axis ring fluxgate magnetometer ${ }^{76}$. The response of the sensor is then converted to the spatial coordinates of the RADPOS probe. The Cartesian (x, y, z) as well as Azimuth coordinates, elevation and angles of rotation of the position sensor are determined during the position tracking ${ }^{74}$. Figure 2.8 shows the coordinate system of the transmitter.

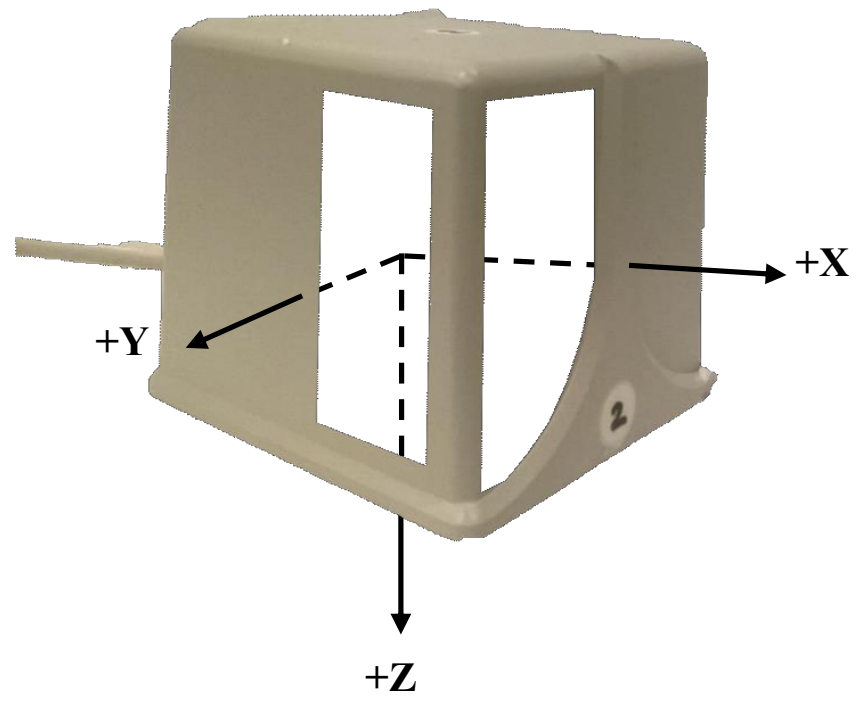

Figure 2.8 The RADPOS DC magnetic field transmitter and its coordinate system. The Cartesian coordinates correspond to the left-right $(X)$, superior-inferior $(Y)$ and anterior-posterior $(Z)$ of the patient. 
The $\mathrm{X}, \mathrm{Y}$, and $\mathrm{Z}$ axes in Figure 2.8 correspond to the left-right, superior-inferior, and anterior-posterior axes of the patient shown previously in Figure 1.3. In each direction a deviation in the recorded position might be observed with increasing the distance between the probe and the transmitter. These deviations are within a few millimeters as long as the distance of the probe from the transmitter stays in the optimal range $(x=15$ to $47 \mathrm{~cm}, \mathrm{y}=$ -21 to $21 \mathrm{~cm}$ and $\mathrm{z}=-10$ to $10 \mathrm{~cm}$ ). Previous work by Cherpak et al. ${ }^{74}$ demonstrated that with this probe-transmitter distance, the accuracy of position measurements with RADPOS in $3 \mathrm{D}$ is better than $2 \mathrm{~mm}$. For larger probe-transmitter separations, recorded positions might need to be corrected accordingly. The position reading stability of RADPOS is verified to be $0.21 \pm 0.07 \mathrm{~mm}$.

Similar to dose measurements, the software allows the position to be logged either manually or automatically at a temporal resolution of as high as $50 \mathrm{~ms}(20 \mathrm{~Hz})$. At this temporal resolution, RADPOS is capable of recording $500 \mathrm{~s}$ of continuous position data points. This is due to the fact that only 10,000 position data points can be logged and saved continuously with RADPOS. Thus, a sampling rate of $100 \mathrm{~ms}(10 \mathrm{~Hz})$ used in this work, seems to be an optimal choice to achieve a data collection time of $1,000 \mathrm{~s}$ for most experiments. 


\section{Chapter 3}

\section{Monte Carlo user codes}

\subsection{EGSnrc user code system}

All Monte Carlo (MC) simulations in this work were performed using the EGSnrc system (V4-2.4.0, 2013, National Research Council of Canada, Ottawa, ON, Canada). The Electron-Gamma-Shower (EGS) system ${ }^{77}$ is a general purpose Monte Carlo package that simulates the transport of electrons and photons in the $\mathrm{keV}$ to $\mathrm{GeV}$ energy range in any element or mixture with atomic numbers between 0 and 100. Physical processes that are modeled in the code include Compton scattering, photoelectric absorption, pair production, bremsstrahlung and positron annihilation. Cross section data for these processes are provided by the user in a separate file (i.e. PEGS4 file). Particle transport simulations are performed using random numbers $\in[0,1]$ that sample known probability distributions to determine the position and type of interactions.

The geometry in which the particle transport happens can range from simple water slabs to complex linac or patient geometries. Particle transport in a voxel continues until the particle leaves the voxel or its energy falls below a cutoff value ECUT (for electron) and PCUT (for photon). Similarly, transport of secondary particles happens if their energy is above a threshold value $\mathrm{AE}$ (for electron) or AP (for photons). Different quantities such as dose might be scored in a voxel as a result of a MC simulation. This entire set of events, from start to termination of a particle transport, is called a particle history. The EGSnrc user code is written in FORTRAN 77 pre-processor language, MORTRAN 3. The diagram shown in Figure 3.1 illustrates the EGSnrc user code system components. 


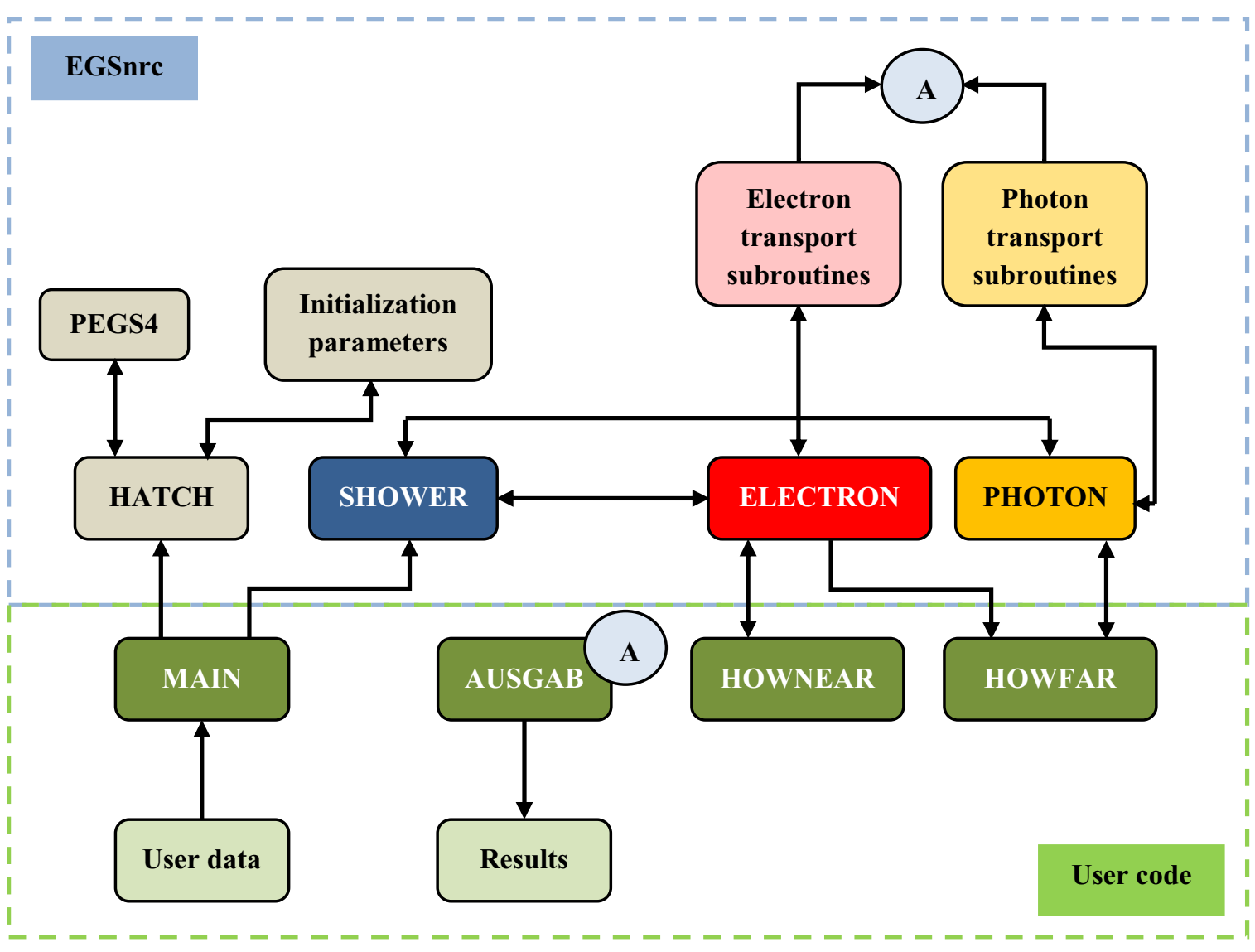

Figure 3.1 Structure of the EGSnrc code system with user codes. Adapted from Kawarkow et al., $2013^{77}$.

An EGSnrc user code is written to model a specific geometry as well as scoring different quantities. It consists of a MAIN subroutine as well as HOWNEAR, HOWFAR and AUSGAB subroutines. Other subroutines might be included to assist with simulations. One of the roles of the MAIN subroutine is to perform initializations for the geometry subroutines, HOWFAR and HOWNEAR. For example, MAIN calls the HATCH subroutine which defines the material properties of the geometries used through a PEGS4 data file. The SHOWER subroutine generates the incident histories and initializes the particle transport. The HOWNEAR and HOWFAR subroutines keep track of which region a particle is in as it is transported through the geometry. HOWNEAR finds the minimum perpendicular distance from current particle position to any boundaries and is used for 
electron transport. HOWFAR finds the distance to the closest boundary along particle's trajectory and from the particle's current position. When HOWFAR is called, the distance calculated through this subroutine is compared to particle's distance to the next interaction position (USTEP) to determine if the particle crosses a boundary or not. Finally, AUSGAB subroutine scores quantities of interest such as energy and dose.

\subsection{Statistical uncertainty analysis}

The uncertainty of a scored quantity in a voxel depends on the number of events that take place in that voxel, where the number of events depends on the number of histories simulated as well as size of that voxel. For the scored quantity $\mathrm{X}$ and when the calculation was done for $\mathrm{N}$ number of histories, the statistical uncertainty $\left(\sigma_{\overline{\mathrm{X}}}\right)$ is calculated according to the following equation ${ }^{78}$ :

$$
\sigma_{\bar{X}}=\sqrt{\frac{1}{N-1}\left(\frac{\sum_{i=1}^{N} X_{i}^{2}}{N}-\left(\frac{\sum_{i=1}^{N} X_{i}}{N}\right)^{2}\right)}
$$

where $X_{i}$ is the scored quantity for history $i$ and $\bar{X}$ is the mean value of $\mathrm{X}$ over all histories. The simplest approach to decrease the uncertainty is increasing number of histories. This, however, increases the computation time. Another approach is increasing the voxel size (i.e. degrading the resolution) which increases the probability of the events occurring in a voxel and as a result decreases the uncertainty.

Throughout this work, the mean relative statistical uncertainty of a dose distribution was calculated according to equation $3.2^{79}$ :

$$
\bar{\sigma}_{D>0.5 D_{\max }}=\sqrt{\frac{1}{n_{D>0.5 D_{\max }}} \sum_{D>0.5 D_{\max }}\left(\frac{\sigma_{k}}{D_{k}}\right)^{2}}
$$


where $\mathrm{n}$ is the number of voxels that receive a dose higher than $50 \%$ of the maximal dose. The number of histories for Monte Carlo simulations was chosen such that the mean relative statistical uncertainties (i.e. $\bar{\sigma}_{D>0.5 D_{\max }}$ ) were calculated to be $0.4 \%$.

\subsection{BEAMnrc, DOSXYZnre and 4DdefDOSXYZnrc user codes}

$\mathrm{BEAMnrc}^{80}$ is an EGSnrc user code designed to model the radiotherapy treatment units including linear accelerators and x-ray tubes. BEAMnrc allows the users to build their own model from component modules (CMs) which describe different geometries. The dimensions and material of each CM are defined in the input file and through the PEGS4 file. The output of a BEAM simulation is the phase space (PS) data which contains the information about the energy, position, direction, weight, and charge of each individual particle crossing a pre-defined scoring plane within the BEAMnrc model.

DOSXYZnrc is an EGSnrc user code that transports incident particles and scores dose in a voxelized Cartesian geometry ${ }^{81}$. The transport geometry used can be a simple rectilinear volume or an egsphant phantom file generated from CT images. The phase space data generated by BEAMnrc can be used as a particle source for DOSXYZnrc simulations. These data might be read from a previously stored phase space file (e.g. Source 2) or generated interactively during DOSXYZnrc simulations (e.g. Source 9) eliminating the need to store a separate phase space file. For the latter, the accelerator modeled by BEAM is compiled as a shared library and particles scored in a scoring plane during an accelerator simulation are sampled simultaneously for use in a DOSXYZnrc simulation ${ }^{81}$.

In this thesis, the DOSXYZnrc user code was used for simulations of the dose delivered to the stationary geometries. To reconstruct the delivered dose to a geometry that is moving/deforming due to respiratory motion, the 4DdefDOSXYZnrc ${ }^{82}$ user code 
was used in this work. The 4DdefDOSXYZnrc user code incorporates the continuous motion and deformation of the dose calculation geometry for each incident particle. When used with Source $21^{83}$, synchronization between the motion of dynamic components of a linac (e.g. MLC) in BEAMnrc, the gantry and collimator rotation as well as the motion/deformations of the patient geometry is enabled. During each history simulation, a randomly generated number (corresponding to a delivered MU fraction) correlates the above beam/dose calculation geometry configurations. As a result, this $4 \mathrm{DMC}$ simulation algorithm models all three effects of respiratory motion previously explained in Chapter 1.

4DdefDOSXYZnrc uses the voxel warping method (VWM) ${ }^{84}$ to accumulate dose deposited on different geometrical states. The motion/deformation of the dose calculation geometry is modeled by displacing/deforming the voxel nodes of the reference geometry using deformation vectors. These vectors may be generated from a motion model or deformable image registration of patient images in different respiratory states. New densities are calculated for each deformed voxel to ensure conservation of mass between the reference (stationary) and deformed geometries. Incident particles are transported and their energy depositions are scored in the deformed geometry. No mapping of dose between geometries is required since the same dose calculation grid is retained between the reference and deformed geometries. The voxel node displacements/deformations are linearly interpolated from the reference to the deformed geometry according to the vectors and a respiratory motion trace. This motion trace is used as a scaling factor (between 0 and 1 or -1 and +1$)$ to determine displacement/deformation magnitude at each respiratory phase. The set of vectors as well as the motion trace are the user provided inputs to the simulation. The output of a 4DdefDOSXYZnrc simulation is the accumulated dose 
distribution in the reference geometry coordinates. This process is demonstrated in Figure

\section{2 .}
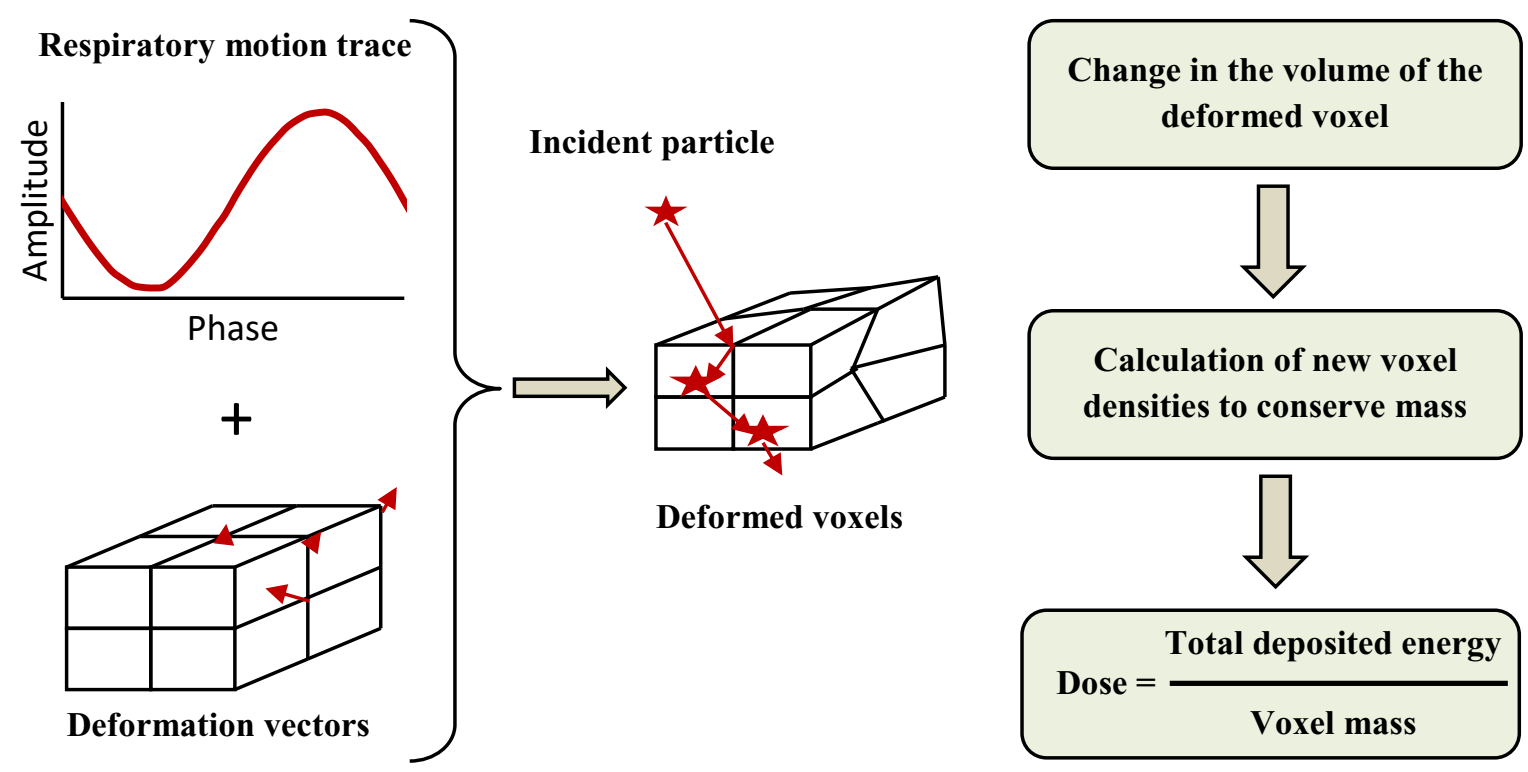

Figure 3.2 Diagram showing how the 4DdefDOSXYZnre user code works based on the voxel warping approach. The input parameters needed to initialize the simulation are shown on the left-hand illustration. The respiratory motion trace functions as the scaling factor for deformation vectors and could be between 0 and 1 or -1 and +1 . The diagram on the right-hand shows a summary of how the dose is calculated during simulation.

The calculated dose from a MC simulation (DOSXYZnrc or 4DdefDOSXYZnrc) represents the dose scored per number of incident particles. Thus, to be compared against measured or TPS calculated dose values, it requires to be converted to absolute dose using the following formulation:

$$
D(c G y)=\frac{(D / \# \text { of incident particles })_{M C \text { individual simulation }}}{(D / \# \text { of incident particles })_{M C \text { calibration simulation }}} \times \frac{1 c G y}{M U} \times M U_{\text {del }}
$$

In this equation $\mathrm{MU}_{\mathrm{del}}$ is the monitor units (MU) delivered by a linac and $\frac{D}{\text { \# of incident particles }}$ is the calculated dose in a MC simulation. In this work the calibration simulation was performed in water for a square field of $10 \times 10 \mathrm{~cm}^{2}$ and source to surface 
distance (SSD) of $100 \mathrm{~cm}$. An SSD is defined as the distance from the beam source to the surface of the patient. The dose was scored at a depth of $10 \mathrm{~cm}$.

For all simulations in this work, the photon cutoff energy (PCUT) and electron cutoff energy (ECUT) were set to 0.01 and $0.7 \mathrm{MeV}$, respectively, and the electron range rejection was set to $2 \mathrm{MeV}$. Bremsstrahlung cross-section enhancement was turned on and the directional bremsstrahlung photon splitting algorithm was used. A photon splitting value of 10 and default values for other transport parameters were used for all DOSXYZnrc simulations. All simulations were performed on the Carleton University Physics Department Research Computer Cluster which consists of 644 cores Intel Xeon CPUs at $2.50-3.00 \mathrm{GHz}$.

\subsection{Dose calculation geometry}

The voxelized geometry for dose calculations might be generated from CT scan images of a phantom or patient. The file that contains the information regarding number of voxels, voxel boundaries, densities and materials for each voxel is called an egsphant file. CT images are generated through 3D or 4DCT scans of the phantom/patient and are stored in the standard format of digital imaging and communications in medicine (DICOM). The CT number (Hounsfield Unit (HU)) to electron density calibration data of the CT scanner allows electron density mapping for each voxel. The CT scanner used in this work was a Big Bore helical CT scanner (Brilliance CT Big Bore, Philips, Amsterdam, Netherlands). The calibration data for the Big Bore CT scanner are shown in Table 3.1. 
Table 3.1 Calibration data for the Big Bore helical CT scanner to map CT numbers (HU) to electron densities.

\begin{tabular}{cc}
\hline CT number & $\begin{array}{c}\text { Relative electron density } \\
\text { (RED) }\end{array}$ \\
\hline-1024 & 0.001 \\
-706 & 0.280 \\
-535 & 0.400 \\
-99 & 0.900 \\
10 & 1.000 \\
208 & 1.090 \\
467 & 1.280 \\
1234 & 1.690 \\
\hline \hline
\end{tabular}

In this work, an in-house software was used to convert CT images to egsphant file and to assign densities and materials to the voxelized geometry. For material assignment to the voxels, the approach introduced by Seco et al. ${ }^{85}$ was followed to define voxels inside the phantom as water with densities derived from the CT images using the calibration data in Table 3.1. All voxels outside the phantom were assigned as air with default density. Since Compton interactions are the dominant source of photon interaction in any medium for photon energies between $100 \mathrm{kV}$ and $10 \mathrm{MV}$, the following approximation can be made:

$$
\mu_{\text {med }} \approx \mu_{m e d}^{C}
$$

where $\mu_{\text {med }}$ and $\mu_{\text {med }}^{C}$ are the total and Compton linear attenuation coefficients for a medium, respectively. For any medium, $\mu_{m e d}^{C}$ is proportional to the electron density of that medium. Thus, the linear attenuation coefficient can be related to $\mu_{w}$ according to following formulation:

$$
\mu_{\text {med }}=R E D_{\text {med }} \times \mu_{w}
$$


In this equation, $\mathrm{RED}_{\text {med }}$ is the relative electron density of the medium with respect to water. This approach enables calculation of $\mathrm{D}_{\mathrm{w}}$ in $\mathrm{MC}$ simulations which allows direct comparison against corresponding measurements with film, RADPOS, etc. The dose calculated with this method. i.e. using REDs was found to be within $1 \%$ of the dose calculated using physical densities.

\subsection{Gamma analysis}

Gamma analysis ${ }^{86}$ is a numerical method to compare the measured and calculated dose distributions where the measurement is used as the reference. The dose-difference and the distance-to-agreement (DTA) combined are used to determine the quality of a calculated dose distribution through gamma analysis. The formalism used to calculate a gamma index is shown in equation 3.5:

$$
\gamma=\min \left\{\sqrt{\left(\frac{r_{m, c}}{\Delta d}\right)^{2}+\left(\frac{\delta_{m, c}}{\Delta D}\right)^{2}}\right\}
$$

where $r_{m, c}$ is the spatial difference between the measurements and calculations for a single measurement point, $\delta_{\mathrm{m}, \mathrm{c}}$ is the difference between the measured and calculated dose values and $\Delta \mathrm{d}$ and $\Delta \mathrm{D}$ are the DTA (in $\mathrm{mm}$ ) and the dose-difference (in \%) criteria, respectively. A gamma analysis passes the defined criteria if $\gamma \leq 1$ and fails otherwise. 


\section{Chapter 4}

\section{Monte Carlo and analytic modeling of an Elekta Infinity linac with Agility MLC: Investigating the significance of accurate model parameters for small radiation fields}

\subsection{Introduction}

\subsubsection{Monte Carlo beam models}

Monte Carlo simulations have proven to be the gold standard for dose calculations and simulations in radiotherapy ${ }^{87,88}$. This is, however, contingent on a beam model that is well-benchmarked against relevant measurements and takes into account the physical properties of the specific linac design.

Regardless of the MC platform used to model the linac, it is critical to adopt a proper procedure to produce an accurate model. The first step to build an accurate MC model of a linac is to create a detailed implementation of the geometry and material of the treatment head components based on the manufacturer provided information. Next, beam parameters (e.g. energy of the initial electron beam that is incident on the target) must be determined through matching simulations of several dosimetric parameters (e.g. percentage-depth-dose curves) against measurements. To obtain the optimal beam model, it is sometimes necessary to adjust the manufacturer provided information (i.e. geometry and material composition) in the model during the benchmarking proccess ${ }^{89}$. Several groups have published descriptions of MC models of linacs from different manufacturers and for different beam energies ${ }^{89-98}$. 
In addition to creating MC models of different linac designs, it is important to have a good understanding of the influence of the model parameters on the accuracy of the beam model. Studies have shown that MC simulations of photon beams are highly sensitive to

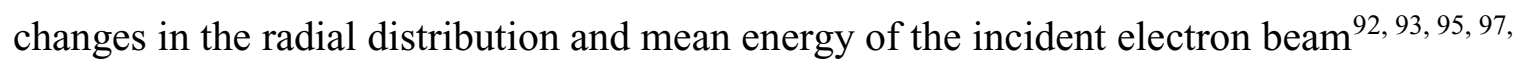
99-101. The shape of the radial intensity profile of the incident electron beam is typically assumed to be Gaussian ${ }^{100}$. In addition to these, the angular divergence of the electron beam is another important parameter to be determined in the beam model of a linac. Besides the parameters of the initial electron beam, the accuracy of MC simulations have shown dependence on the geometry, shape and material of several linac components such as target and collimation system ${ }^{89,93,97}$.

\subsubsection{Virtual source models}

Although modeling treatment beams using MC simulations is the most accurate approach, it is not the most efficient. Due to simulating large number of particles as well as wide range of particle transports and interactions, full $\mathrm{MC}$ calculations (i.e. beam and dose calculations) usually require many CPU-hours to complete. Thus, less complex and yet accurate approaches to model the treatment beam could be valuable to achieve more efficient and faster calculation times. Virtual source models (VSMs) typically include multiple virtual sources that utilize the phase space (PS) data to simulate the beam contributions from different components of the treatment head. The three main components of any VSM include: 1) primary photon source to model photons generated in the target, 2) secondary photon source to model photons scattered from the primary collimator, flattening filter and the rest of the linac head components, 3) electron contamination source ${ }^{102-107}$. The necessary PS data are derived from full MC simulations of the treatment 
head and scored in specific planes, keeping track of the contributions from each linac component. The location of all three sources need to be defined in the VSM according to the PS data. This becomes difficult for the secondary photons and the contamination electron source due to the multiple sources that produce these radiations. Similar to MC beam models, parameters defined in a VSM (e.g. virtual source size, energy fluence, weight of each source) need to be finely tuned against MC simulations and/or measurements. Studies show that accurate modeling and tuning of the size and contribution of the primary photon source is a critical step to an accurate virtual source model ${ }^{108}$.

The main advantage of the VSMs compared to MC simulations is their higher efficiency in calculation times. Rather than exact simulation of particle transport, they use a simplified analytic approach based on mathematical functions for all relevant beam calculations. This makes them a robust beam model to be implemented in treatment planning systems. The beam model implemented in Monaco treatment planning system (Elekta AB, Stockholm, Sweden) for Elekta linear accelerators is a virtual source model and comprises all three sources in a typical VSM ${ }^{105}$. The primary source is Gaussianshaped with fixed spatial radial distribution. The two other sources are designed to have a Gaussian distribution as well but with spatial radial distributions that depend on the energy of the particles used to model the source.

\subsubsection{MLC modeling}

Radiation fields delivered using 3D-CRT, IMRT and VMAT treatment techniques are shaped by the MLC. These techniques are associated with high precision conformal radiation fields to treat a target volume while sparing the OAR. As a result, appropriate modeling of the MLC parameters becomes very important for accurate dose calculations 
using any beam model. Several research groups have studied the importance of including and tuning parameters of the MLC including its geometry (i.e. rounded leaf ends, tongue \& groove and leaf bank rotation/defocusing), material, transmission properties and interleaf leakage in $\mathrm{MC}^{109-115}$ and virtual source ${ }^{116-118}$ beam models. Similar to source parameters, properties of the MLC can be tuned according to appropriate dosimetric measurements. In Monaco, the MLC is modeled as a transmission probability filter containing a series of planes through which particles from the virtual sources are traced and attenuated. Transmission probabilities are determined based on the path length of particles through these planes, physical and geometrical properties of the MLC and transmission values associated with different parts of the filter.

\subsubsection{Small fields in radiation therapy}

The region past the build-up region in broad radiation fields is where transient charged particle equilibrium (TCPE) occurs in both longitudinal (i.e. along the beam central axis) and lateral (i.e. lateral of the beam central axis) directions. This helps establish well-conditioned dosimetry measurements for photon beams in this category. However, small radiation fields are often delivered during IMRT, VMAT and stereotactic body radiation therapy (SBRT) techniques. A small radiation field is defined to have dimensions comparable or less than the lateral range of charged particles ${ }^{119}$. Because the range of charged particles decreases with decreasing beam energy, a lower bound can be defined for which a field size is called small for each photon energy. For a $6 \mathrm{MV}$ photon beam, this threshold is defined to be $3 \times 3 \mathrm{~cm}^{2} 119,120$. In addition, due to a lack of lateral charged particle equilibrium (LCPE), occlusion of the radiation source by the collimation system is another issue associated with small radiation fields. The result of such source occlusion is 
an output that is measured to be lower than compared to conventional large field sizes. Another observed effect of the source occlusion is a widened dose profile which results in an overestimated field size compared to the collimator settings ${ }^{119,121,122}$. Under this condition, the full-width-at-half-maximum (FWHM) of the dose profile does not agree with the field size defined by collimator opening. Figure 4.1 illustrates source occlusion and its impact on the measured dose profile for a small field in comparison to a large field size.

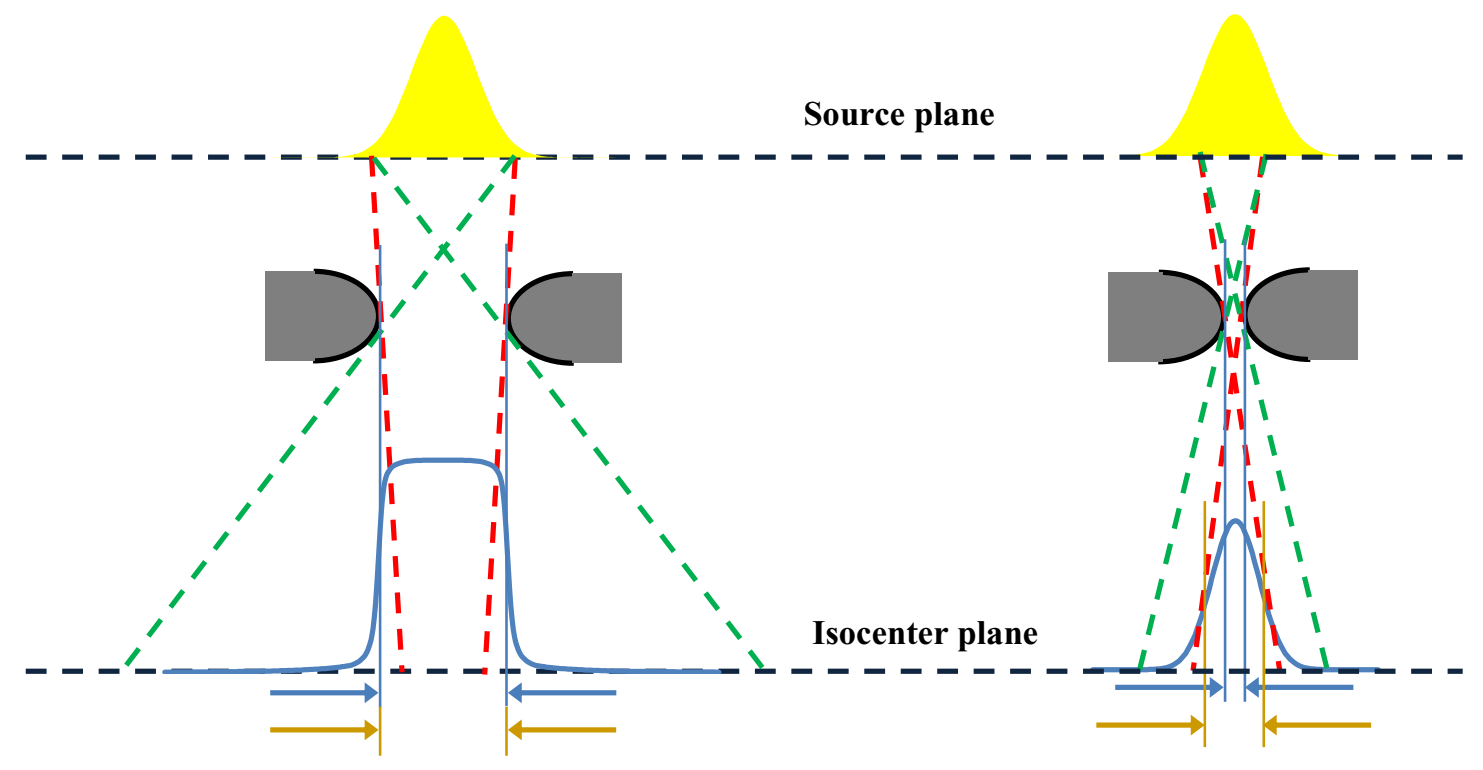

$\longleftrightarrow$ Actual field size setting

$\longleftrightarrow$ Field size measured from dose profile based on its FWHM

Figure 4.1 Illustration of source occlusion and the resultant dose profile. If the field size is large enough (left), beam source is fully viewed at the isocenter plane and the resulting dose profile measures the correct field size. For very small field sizes (right), beam source is partially viewed at the isocenter plane which yields a widened dose profile that measures an overestimated field size due to a larger FWHM. Adapted from IPEM Report 103, 2010 ${ }^{123}$ and Das et al., 2008 ${ }^{119}$.

Since small radiation fields are characterized by lateral charged particle disequilibrium and source occlusion, they require a different dosimetry formalism than the ones used for larger fields. One element is the detector size (e.g. ion chambers and diodes) 
which plays a critical role in dosimetry of small radiation fields. Small size detectors reduce the volume averaging effect over the volume of the detector which results in more accurate dose measurements in small radiation fields ${ }^{119,}, 122$.

Due to these characteristics, beam models simulating small radiation fields require special tuning of the primary photon source size in order to avoid large discrepancies between simulated and measured dose profiles ${ }^{108,}{ }^{124}$. Sikora et al. ${ }^{108}$ showed that to achieve a good agreement between calculated and measured cross- and in-line profiles for a $0.8 \times 0.8 \mathrm{~cm}^{2}$ field, the FWHM of the primary photon source needs to be reduced by at least $30 \%$ from its original value determined for larger field sizes. If appropriately modeled, simulations of small fields can be helpful to derive correction factors required for detectors used for measurements ${ }^{125-128}$ as well as for dose calculations of treatment plans consisting of small fields $88,96,109,121,125,129,130$.

This chapter presents, for the first time, a detailed MC model of an Elekta Infinity linac with Agility MLC leaves, created in EGSnrc/BEAMnrc and benchmarked against appropriate measurements. In addition, an analytic model of this linac using multiple virtual sources was created to investigate the impact of different beam parameters on the photon fluence at the isocenter plane. Finally, results from the analytic model and EGSnrc simulations are used to explain the dose discrepancies observed between dose profiles obtained from Monaco calculations and measurements for a small field (alternating openclosed MLC leaves). 


\subsection{Materials and Methods}

\subsubsection{BEAMnrc model of an Elekta Infinity linear accelerator}

A detailed model of an Elekta Infinity linac with Agility MLC was created using BEAMnrc. The linac model was constructed for $6 \mathrm{MV}$ energy, based on the technical data provided by the manufacturer and previously published work ${ }^{96}$ to adjust the simulation parameters for megavoltage photon beams. Figure 4.2 shows a geometrical illustration of this model including the patient independent (target, primary collimator, flattening filter, monitor ion chamber and backscatter plate) and patient dependent (160 MLC leaves, lower jaws) components.

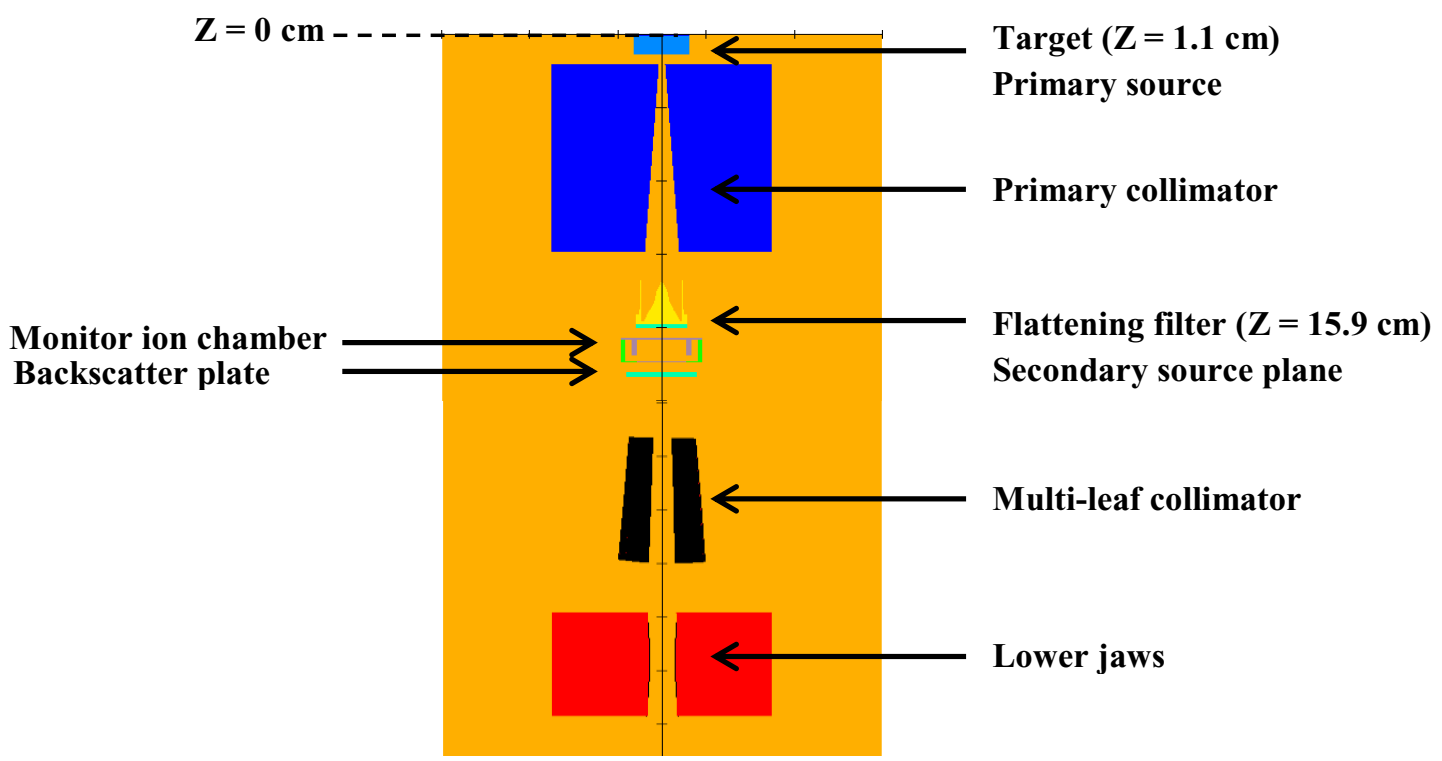

Figure 4.2 BEAMnrc preview of the Elekta Infinity linac model with Agility MLC showing the various components.

To model the multi-leaf collimator and lower jaws, the SYNCMLCE and SYNCMLCQ CMs were used, respectively. The 'SYNC' versions of these component modules enable synchronization of the motion of the MLC, gantry and jaws in the linac model as well as dose calculation geometry by using a common, randomly generated MU 
index which lies between 0 and 1, to sample the configuration of the linac components for each particle history. A 'SYNC' version of the MLCQ component module was created by modifying this CM to read the MU index generated in the SYNCMLCE CM. Source $19^{131}$ was used as the particle source in BEAMnrc to define the incident electron beam. Source 19 is an elliptical beam source with Gaussian intensity distributions defined in X (crossline) and Y (in-line) directions, with either parallel or angular spread. The MLC density, composition, and inter-leaf air gap were initially set to the values specified by the manufacturer.

One feature of the Agility MLC is defocusing of the leaf bank (i.e. leaf bank rotation) rather than using a tongue $\&$ groove to reduce the inter-leaf leakage. In-plane views of a sample defocused MLC leaf bank vs focused with tongue $\&$ groove mechanism are shown in Figure 4.3. The resultant leaf bank rotation causes a translation in the center of the field opening at the isocenter. The appropriate shift, calculated by equation 4.1, was applied to the SYNCMLCE component in the beam model of the linac to account for this translation.

$$
\text { shift } t_{M L C}=\frac{\text { Leaf thickness } \times \sin (\text { LBROT })}{2}
$$




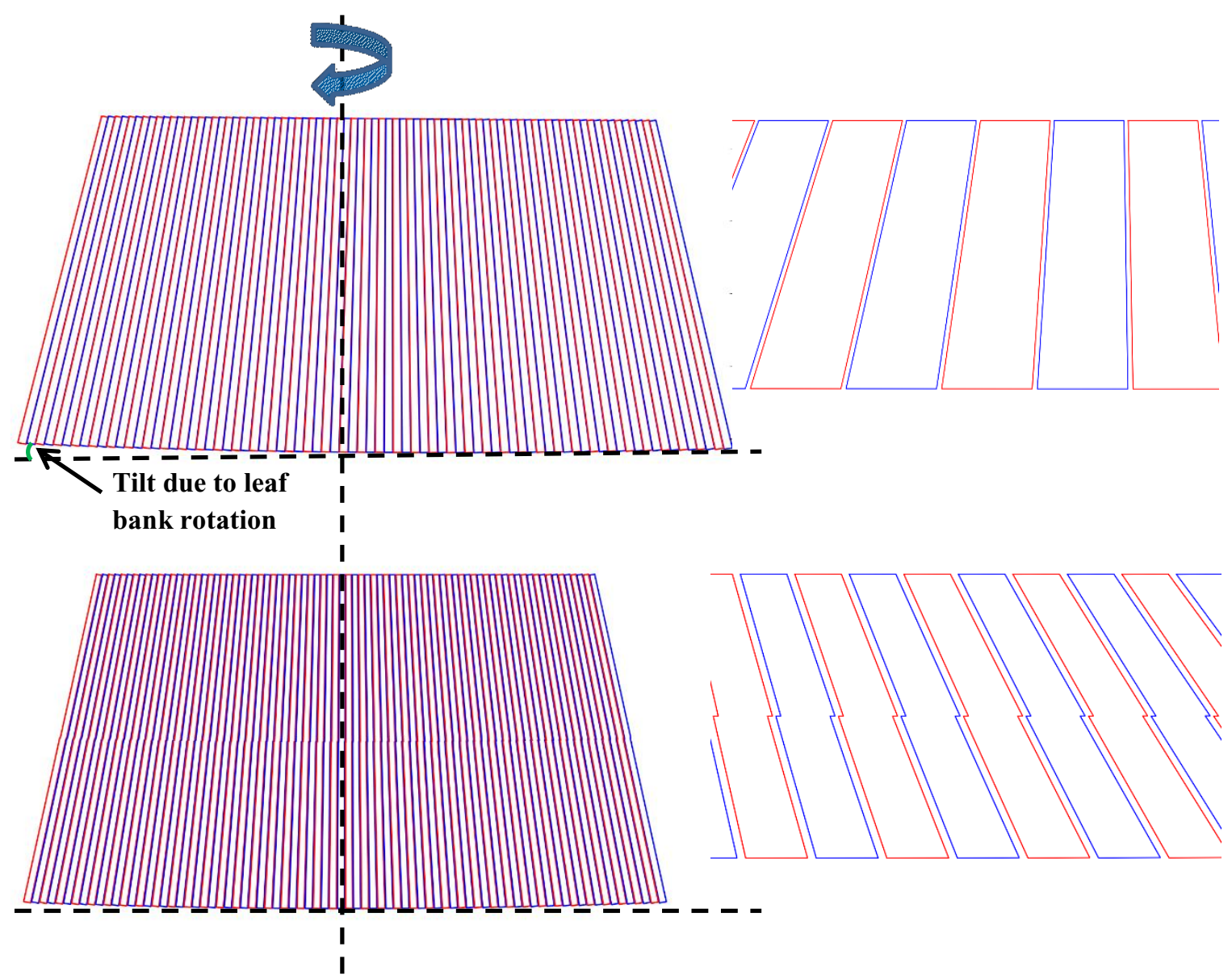

Figure 4.3 In-plane view of MLC leaf banks with defocusing (top left) vs focused with tongue \& groove mechanism (bottom left) to reduce inter-leaf leakage. Zoomed views are also shown (top right and bottom right).

All measurements of output factors, percentage-depth-dose (PDD) and cross- and in-line profiles were performed in a water tank (Blue Phantom², IBA Dosimetry, Schwarzenbruck, Germany) on an Elekta Infinity linac operating at a nominal dose rate of $600 \mathrm{MU} / \mathrm{min}$. The output factor, also known as the relative dose factor (RDF) is defined as the ratio of the dose at depth $\mathrm{z}$ in a phantom for a given field size to the dose at same depth for a reference field size (i.e. $10 \times 10 \mathrm{~cm}^{2}$ ). Both measurements should be performed for the same photon beam at a given SSD. The PDD is another relative dosimetric parameter that 
characterizes variation of dose with depth along the beam central axis. It is defined as the ratio of the dose at a depth $\mathrm{z}$ in the phantom in a given field to the dose at $\mathrm{Z}_{\max }$ where the dose reaches its maximum. The off-axis ratios (OAR) are dose profiles measured perpendicularly to the beam central axis at a given depth in a phantom. They are defined as the ratio of the dose at an off-axis point to the dose on the central axis at the same depth. In this work, cross-line and in-line profiles refer to the OARs measured parallel and perpendicular to the MLC leaves, respectively.

For output factor measurements $100 \mathrm{MU}$ was delivered for each field size. Measurements for small field sizes were performed using an Exradin A16 ionization chamber (Standard imaging Inc., Middleton, WI, USA) as well as RFD photon diodes (IBA Dosimetry, Schwarzenbruck, Germany). For larger field sizes, a CC13 ionization chamber (IBA Dosimetry, Schwarzenbruck, Germany) was used. The collecting volumes of the Exradin A16 and CC13 ion chambers are 0.007 and $0.13 \mathrm{~cm}^{3}$, respectively. PDD and profiles were measured using CC13 ionization chamber while build-up and penumbra regions were measured using RFD photon diodes. All measurements were performed at an SSD equal to $100 \mathrm{~cm}$. PDD curves were measured for a $5 \times 5 \mathrm{~cm}^{2}$ field size while output factors as well as cross- and in-line profiles were measured for various small and large field sizes $\left(2 \times 2 \mathrm{~cm}^{2}\right.$ up to $\left.40 \times 40 \mathrm{~cm}^{2}\right)$ at $5 \mathrm{~cm}$ depth.

In order to extract the leaf bank rotation (LBROT) value a field with alternating open-closed MLC leaf pairs was created in Monaco V.5.11.01. Dose profiles for this small field size (i.e. $5 \mathrm{~mm}$ along the in-line/leaf bank rotation direction) are highly sensitive to the value of LBROT. Two sections with 5 adjacent leaves open or closed (i.e. $2.5 \mathrm{~cm}$ along the in-line and $10 \mathrm{~cm}$ in the cross-line directions) were included in the pattern as well to 
compare dose values for larger field sizes. A beam's eye view (BEV) of the described field is shown in Figure 4.4.

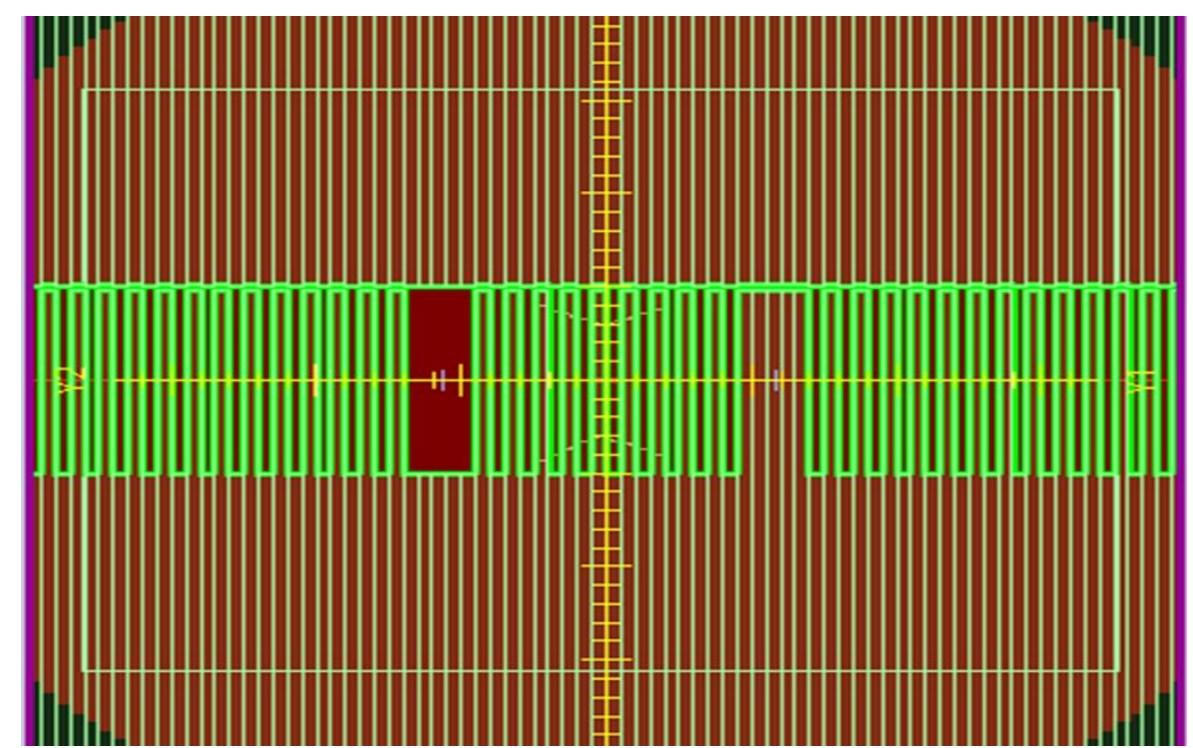

Figure 4.4 BEV of the fields constructed to evaluate the LBROT value. The fields consist of a small field size (1-open leaf) to verify leaf bank rotation and one larger field size (5-open leaves) for dosimetric verification.

Dose profiles for this field were measured using calibrated Gafchromic film. Point doses were measured using an A1SL ionization chamber (Standard imaging Inc., Middleton, WI, USA) at the center of the 5-open and 5-closed leaves sections, $5.75 \mathrm{~cm}$ laterally from the isocenter. The A1SL chamber has a collecting volume of $0.053 \mathrm{~cm}^{3}$. Dose measurements were performed at a source to axis distance (SAD) of $100 \mathrm{~cm}$ at a depth of $5 \mathrm{~cm}$ (beam isocenter) in a $30 \times 30 \times 10 \mathrm{~cm}^{3}$ Solid Water (RMI457 Gammex, Wisconsin, USA) phantom. In an SAD setup, the point of measurement (e.g. target volume) is placed at the linac isocenter and the SAD is defined as the distance between the beam source and isocenter.

The energy of the mono-energetic electron beam (i.e. Source 19) was determined by matching PDD curves from measurements and MC calculations for a $5 \times 5 \mathrm{~cm}^{2}$ field. 
Penumbrae of measured and simulated profiles in both cross- and in-line directions were matched to extract the FWHM value of the electron beam source along both directions independently. Small fields of sizes $2 \times 2 \mathrm{~cm}^{2}$ and $5 \times 5 \mathrm{~cm}^{2}$ were chosen because dose profiles of small fields are less sensitive to the mean angular divergence of the initial electron beam. ${ }^{95}$ The mean angular divergence of the electron beam was adjusted by comparing calculated and measured dose profiles of large fields (i.e. $30 \times 30 \mathrm{~cm}^{2}$ and $40 \times 40 \mathrm{~cm}^{2}$ ). The FWHM value was then further tuned by matching measured and simulated RDFs.

As for the LBROT, measured dose profiles for the alternating open-closed MLC pattern were compared to simulations with varying LBROT values until the best agreement between measurements and simulations was found.

The value of the shift calculated by equation 4.1 was confirmed by calculating the offset between the center of the open fields of sizes $1 \times 5,5 \times 5$ and $15 \times 15 \mathrm{~cm}^{2}$ from MC simulations and measurements. Measurements were performed at SAD equal to $100 \mathrm{~cm}$ and $100 \mathrm{MU}$ was delivered to the film at $5 \mathrm{~cm}$ depth of the solid water.

The initial values of the MLC density, composition, and inter-leaf air gap were adjusted to find an agreement between calculated and measured leaf transmission values utilizing the field shown in Figure 4.4 and according to the explained procedure. Leaf transmission was measured using ion chamber measurements at the positions of 5open, 5-closed leaves, respectively. The inter-leaf air gap was then tweaked until the best agreement between measurements and simulations was found for the single leaf openings in the same field. Once inter-leaf air gap value is modified, the leaf transmission changes. As a consequence, the MLC density and composition as well as the inter-leaf air gap 
were adjusted again to match the measured leaf transmission value. The process was then repeated until no improvements were observed.

\subsubsection{Analytic and virtual source model of the Elekta Infinity linac}

An analytic model of the Elekta Infinity linac was created in Python as a simple and fast method to assist with understanding the impact of different input parameters on the photon fluence at the isocenter plane from small radiation fields. The model parameters were tuned by comparison against MC simulations using the validated BEAMnrc model of the Elekta Infinity linac as described previously. The analytic model consists of two virtual photon sources (primary and secondary) as well as a model of the Agility MLC leaves. In order to extract parameters for the analytic model, the BEAMnrc user code was modified to score photon fluence and position as well as other photon characteristics (energy and angular distributions) at different planes of the MC model of the linac. This helped to avoid saving large PS files that contain large number of particles to extract the needed information.

The primary photon source was used to model the bremsstrahlung photons generated in the target. In the MC linac model, photons were scored below the flattening filter, $Z=15.9 \mathrm{~cm}$ from the reference plane $(Z=0 \mathrm{~cm})$, and back projected to the distal side of the target at $Z=1.1 \mathrm{~cm}$. These planes are shown on Figure 4.2. Only photons whose projected trajectories intersected the isocenter plane $(Z=100 \mathrm{~cm})$ within the in-line distance of $\pm 10 \mathrm{~mm}$ from the isocenter were included in the primary source. These limits were chosen based on the small field size $\left(5 \times 10 \mathrm{~mm}^{2}\right.$ at isocenter $)$ and the fact that bremsstrahlung photons coming out of the target are fairly forward-peaked. The resultant photon fluence was found to fit a Gaussian distribution with a FWHM $=1.05 \mathrm{~mm}$. 
The secondary photon source was created to model the scattered photons coming from the primary collimator and flattening filter and was located at the distal plane of the flattening filter at $Z=15.9 \mathrm{~cm}$ (as marked on Figure 4.2). A photon elimination criterion of $\pm 7 \mathrm{~cm}$ in the in-line distance was adopted to score photons for the secondary source. Both virtual sources are shown in Figure 4.5.
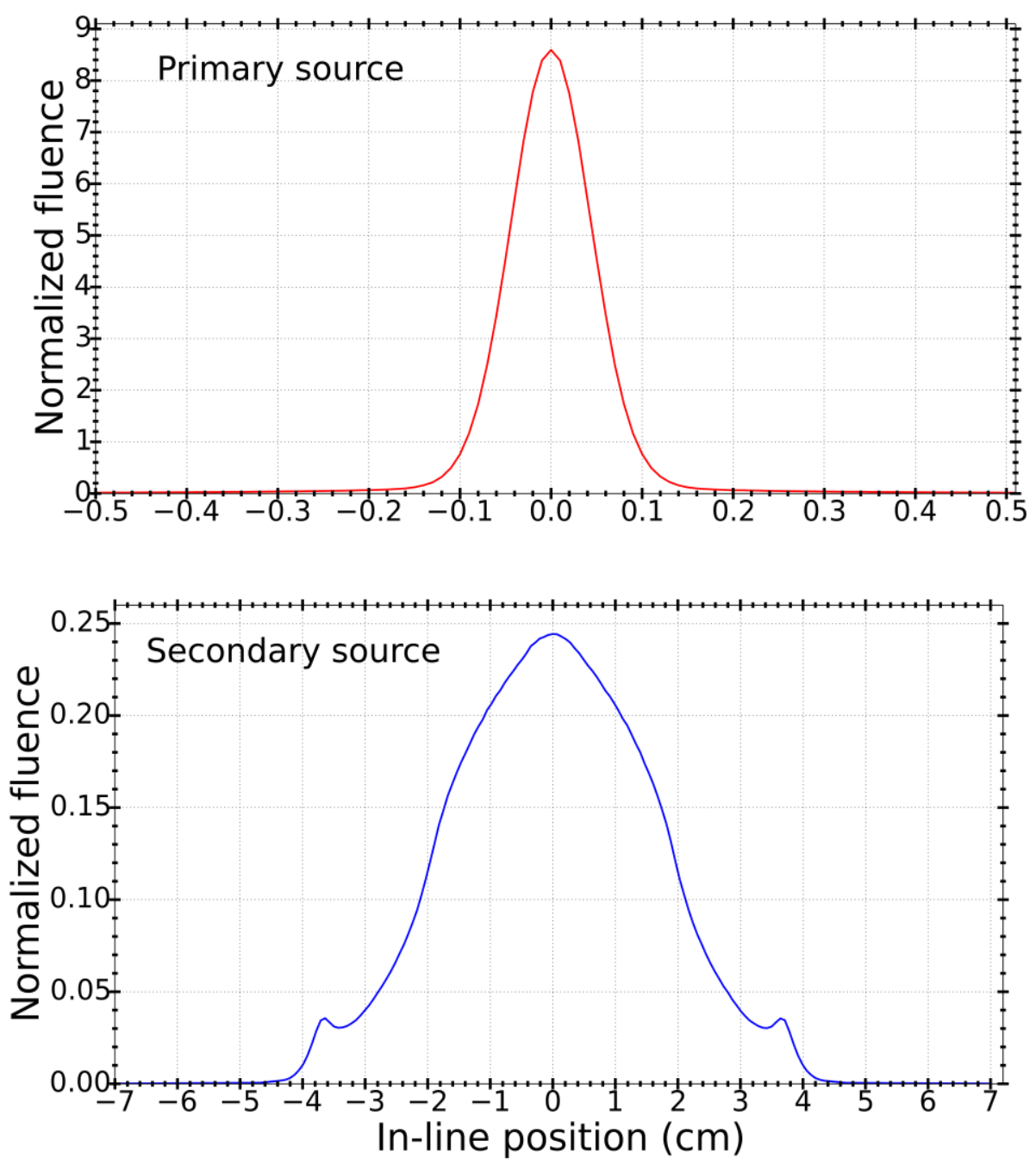

Figure 4.5 Primary (top) and Secondary (bottom) virtual photon sources used in the analytic model. The primary and secondary photon sources are placed at $Z=1.1 \mathrm{~cm}$ and $Z=15.9 \mathrm{~cm}$ from the reference plane $(Z=0 \mathrm{~cm})$, respectively. 
In order to calculate fluence profiles at the isocenter plane, a ray tracing algorithm was used to trace the trajectories of photons from both virtual sources through the MLC. The detailed geometry of the Agility MLC leaves, including the leaf bank rotation, was adopted into the analytic model. The source boundaries for the isocenter fluence calculation comprise the points of non-zero fluence plus an additional $5 \mathrm{~mm}$ margin. Attenuation of photons in the MLC leaves was modeled by exponential attenuation. Attenuation coefficient data for the leaf material composition were obtained from NIST XCOM database using the average photon energy distribution obtained from $\mathrm{MC}$ simulations for each virtual source. The contribution of each virtual source to the resultant photon fluence at the isocenter plane was derived from the ratio of the number of photons from each source to the total number of photons at the isocenter plane from MC simulations. Figure 4.6 illustrates the ray tracing process for fluence calculation using the analytic model.

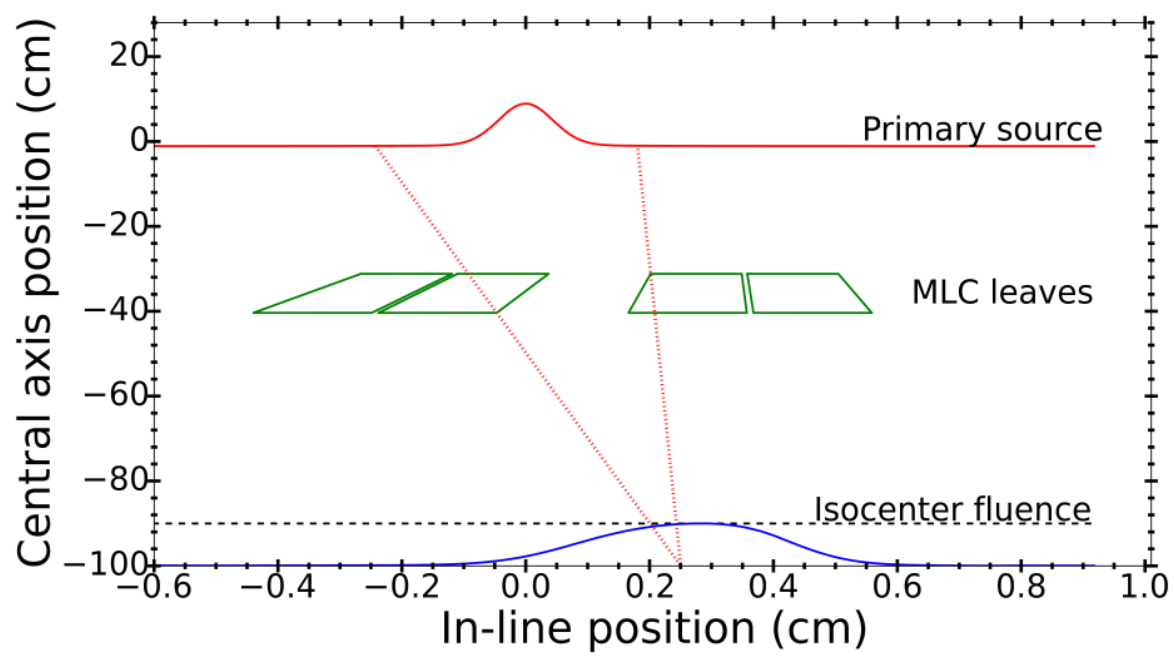

Figure 4.6 Ray diagram illustrating the photon fluence calculation process of the analytic model. The fluence at each point along the in-line position on the isocenter plane is the integral of the source. The source boundaries are shown by the photon rays tracing from the isocenter to the source plane. 


\subsubsection{Impact of analytic model parameters on the fluence at the isocenter plane}

The impact of the analytic model parameters on the isocentric photon fluence for a single open leaf field was studied. LBROT, the size of the primary photon source, angular and energy distributions and attenuation in the MLC leaves were modified and their impact on the fluence was investigated. Moreover, since the MLC model in Monaco also includes a tongue $\&$ groove design (the Monaco beam model parameters include a tongue $\&$ groove width), the impact of the tongue \& groove parameters on the fluence was studied using the analytic model. Finally, the impact of exclusion of the secondary photon source was investigated. It was expected that the secondary photon source would have limited impact due to the fact that head scatter decreases significantly as a result of source occlusion at small field sizes ${ }^{124}$. Fluence profile changes were quantified in terms of maximum photon fluence, integral of the fluence profile and distance-to-agreement (DTA) at the penumbra region ( $50 \%$ of the maximum fluence).

\subsubsection{Comparison of Monaco and EGSnrc dose calculations}

Dose profiles from measurements, Monaco and EGSnrc calculations were compared for the alternating open-closed MLC pattern field in Figure 4.4. The voxelized geometry for dose calculation was defined according to the geometry and material of the solid water phantom used for dose measurements in Section 4.2.1. The voxel size was defined to be $1 \times 1 \times 1 \mathrm{~mm}^{3}$ to match the resolution of film measurements. For dose calculations using DOSXYZnrc, the linac model described in Section 4.2.1 was used as a particle source (Source 9) thus eliminating the need to store a separate phase space file. A photon splitting value of 40 and other transport parameters as described in Section 3.3 of Chapter 3 were used for all DOSXYZnrc simulations. Dose was calculated at $5 \mathrm{~cm}$ depth 
of the voxelized geometry phantom and same beam settings as explained in Section 4.2.1. Dose calculations were performed with $2 \times 10^{9}$ histories.

Monaco utilizes the X-ray voxel Monte Carlo (XVMC) $)^{132,133}$ algorithm for dose calculations. The $\mathrm{XVMC}$ is a fast MC simulation algorithm for dose calculation purposes for clinical treatment planning routines. Dose calculation for the XVMC is approximately 15-20 times faster than the DOSXYZnrc. This improved calculation speed is mainly achieved by history repetitions as well as simplifications and approximation in the electron and photon transport algorithms. History repetitions works by using same electron history in different regions of the dose calculation geometry. Approximations made to the transport algorithm include discarding secondary bremsstrahlung photons and neglecting some multiple scattering events as well as photoelectric effect. Such approximations make this model applicable only to the situations that are typically used in radiotherapy, i.e. electron energy range (1-30) $\mathrm{MeV}$ and low $\mathrm{Z}$ material with density range $(0-3) \mathrm{g} / \mathrm{cm}^{3}$.

For dose calculations in Monaco, a $1 \mathrm{~mm}$ dose calculation grid with a statistical uncertainty of $1 \%$ was chosen. Details of the beam model for the Elekta Infinity linac and Agility MLC in Monaco were used to change source parameters (FWHM of the incident electron source) and geometry of the MLC leaves (inclusion of tongue \& groove with groove width of $0.4 \mathrm{~mm}$ ) in the BEAMnrc model of the linac.

\subsubsection{Comparison metrics}

For comparison purposes, point dose differences between EGSnrc and Monaco calculated dose values were determined. These difference comparisons were quoted as the percentage of the EGSnrc simulated dose values at the point of comparison. Similar comparisons were performed between Monaco/EGSnrc calculations and measurements 
with the difference comparisons defined as the percentage of the measured dose values at the point of comparison. To compare dose profiles from EGSnrc simulations and measurements, a $1 \mathrm{D}$ global gamma analysis with various criteria (i.e. $1 \% / 1 \mathrm{~mm}$ and $2 \% / 1$ $\mathrm{mm}$ ) was utilized with measurement dose used as the reference.

\subsection{Results}

\subsubsection{BEAMnrc model of an Elekta Infinity linear accelerator}

Comparisons between the measured and simulated PDDs, OARs and RDFs are shown in Figures 4.7-4.9. Excellent agreement was observed between measured and simulated PDD curves as illustrated in Figure 4.7 (top). All dose points past the build-up region passed a 1\%/1 mm gamma comparison. Also, over $90 \%$ of dose points from $\mathrm{MC}$ simulations were found to be within $0.5 \%$ of measurements as shown in Figure 4.7 (bottom). 

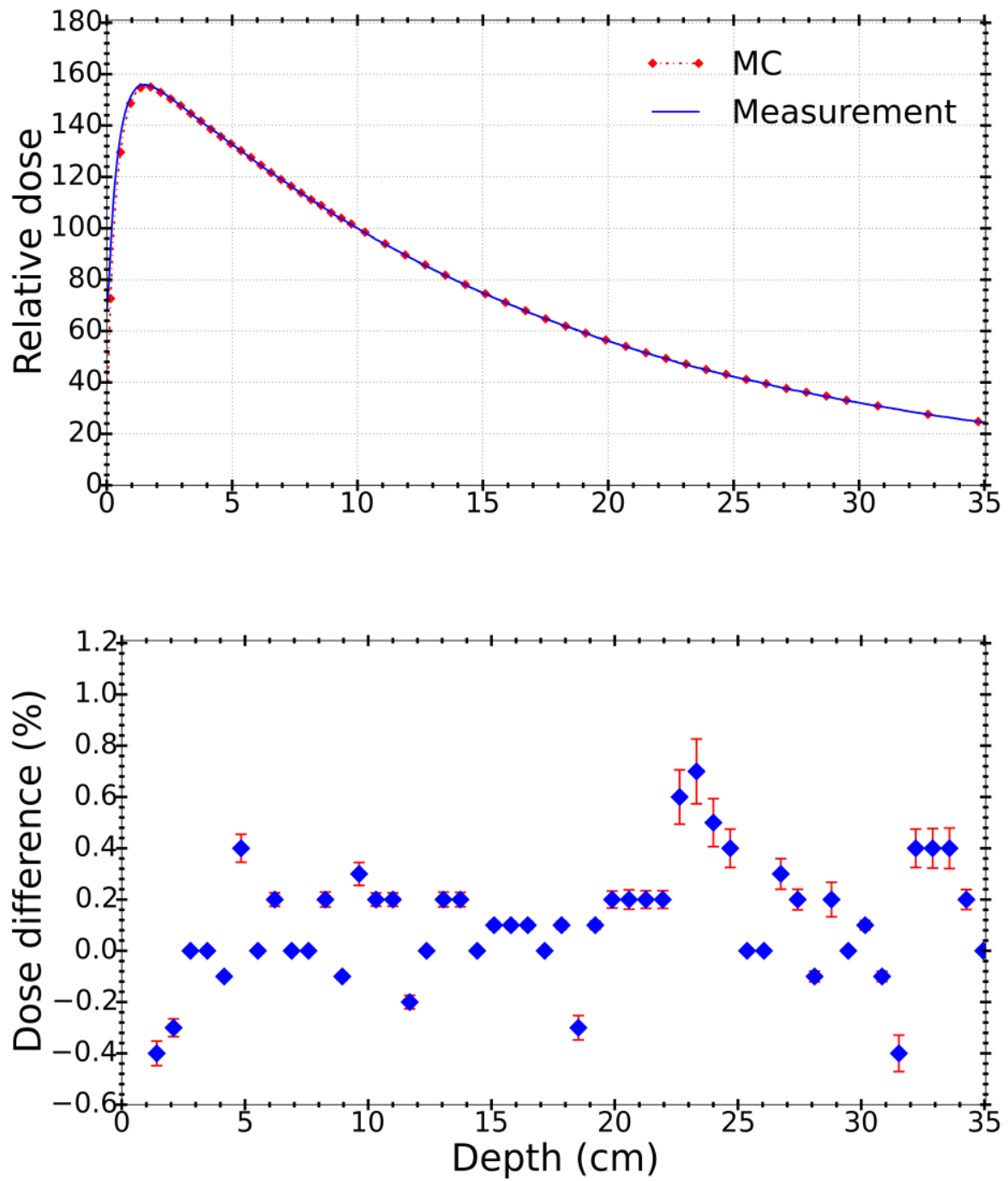

Figure 4.7 Comparison of the measured and calculated PDD curves for a $5 \times 5 \mathrm{~cm}^{2}$ field size at SSD $=100 \mathrm{~cm}$ normalized at $10 \mathrm{~cm}$ depth (top) and percent dose differences for calculated point doses against measurements (bottom). Error bars represent statistical uncertainty from MC simulations (0.1\%).

The cross-line and in-line profiles as presented in Figure 4.8 (a) and 4.8 (b) also showed good agreement between measurements and MC calculations. For cross-line profiles, all points from $\mathrm{MC}$ calculations passed a $2 \% / 1 \mathrm{~mm}$ gamma analysis when compared against measurements. For the same criteria applied to in-line profiles, passing rates of $100 \%$ and over $95 \%$ were observed for field sizes smaller than or equal to 
$20 \times 20 \mathrm{~cm}^{2}$ and larger than $20 \times 20 \mathrm{~cm}^{2}$, respectively. Average DTA (left and right) values between MC calculated and measured data, in penumbra region (50\% of the relative dose), for field sizes from $2 \times 2 \mathrm{~cm}^{2}$ to $40 \times 40 \mathrm{~cm}^{2}$ were found to be better than $0.1 \mathrm{~mm}$.

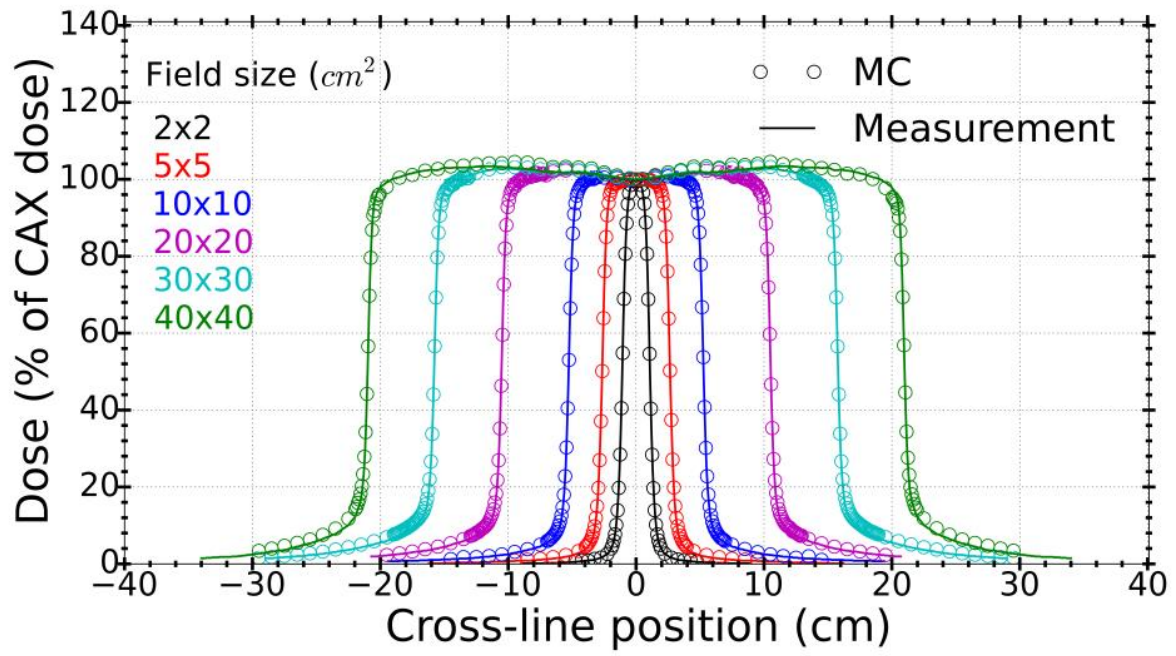

(a)

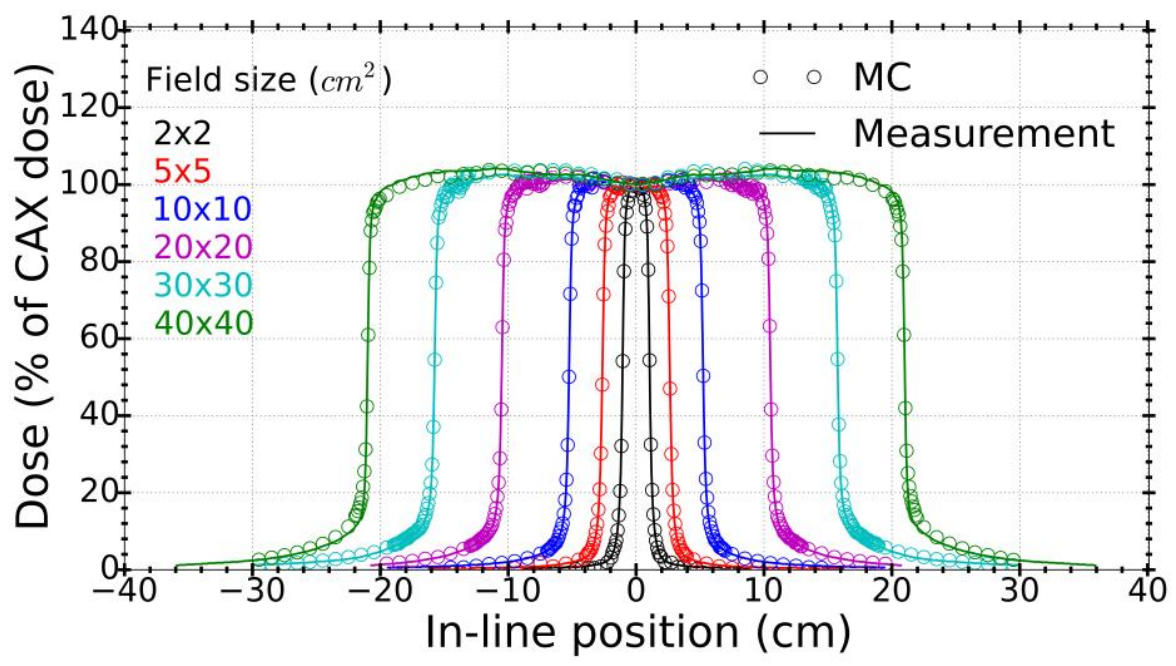

(b)

Figure 4.8 Comparison of the measured and calculated (a) cross-line and (b) in-line profiles for various field sizes at $5 \mathrm{~cm}$ depth and $100 \mathrm{~cm}$ SSD. 
Figure 4.9 shows a comparison of measured and MC calculated RDFs for several field sizes. The agreement was found to be very good for all field sizes, with the largest discrepancy of less than $0.5 \%$ for the $40 \times 40 \mathrm{~cm}^{2}$ field size.

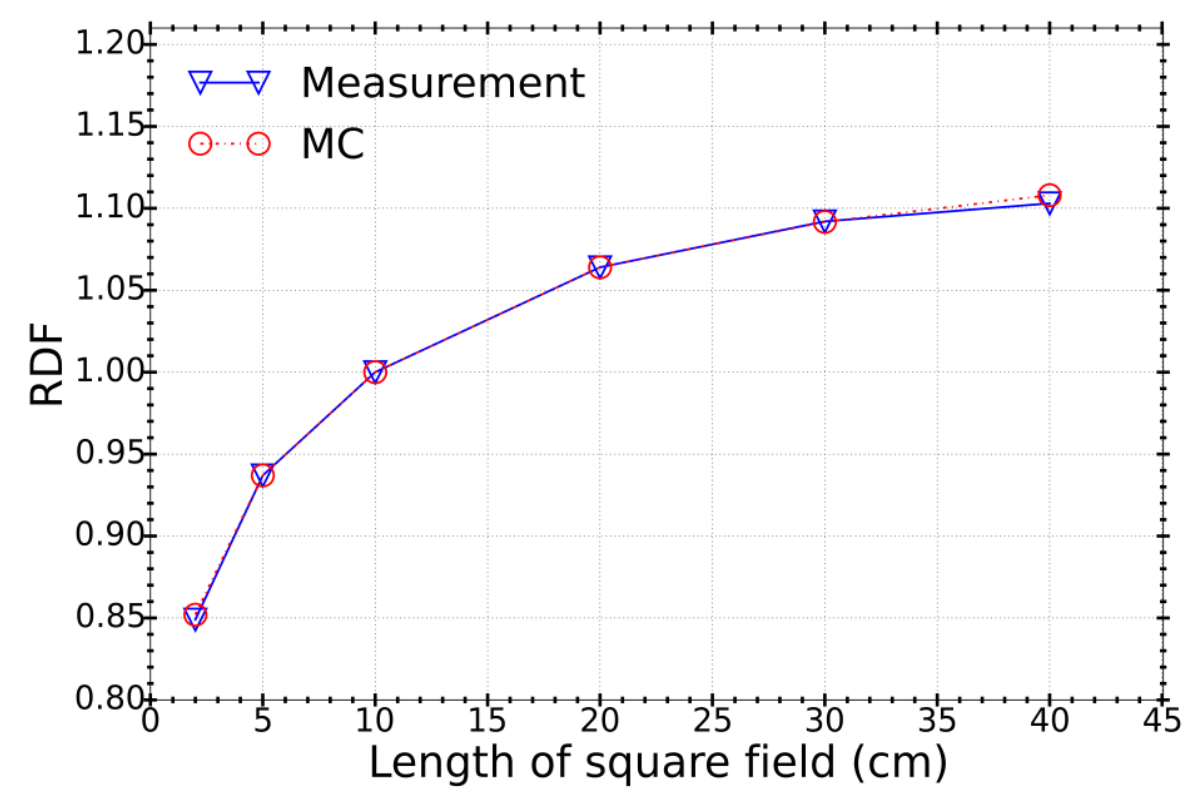

Figure 4.9 Comparison of the measured and calculated relative output factors (RDFs) for various field sizes at $5 \mathrm{~cm}$ depth and $100 \mathrm{~cm}$ SSD.

Figure 4.10 shows the measured and simulated dose profiles for the alternating leaf field for LBROT values of $0,6,9$ and $12 \mathrm{mrad}$. Percentage dose differences at the maxima (corresponding to 1-open leaf) and minima (corresponding to 1-closed leaf) as well as DTA values at the penumbra region (50\% of the maximum dose) are shown in Table 4.1. 


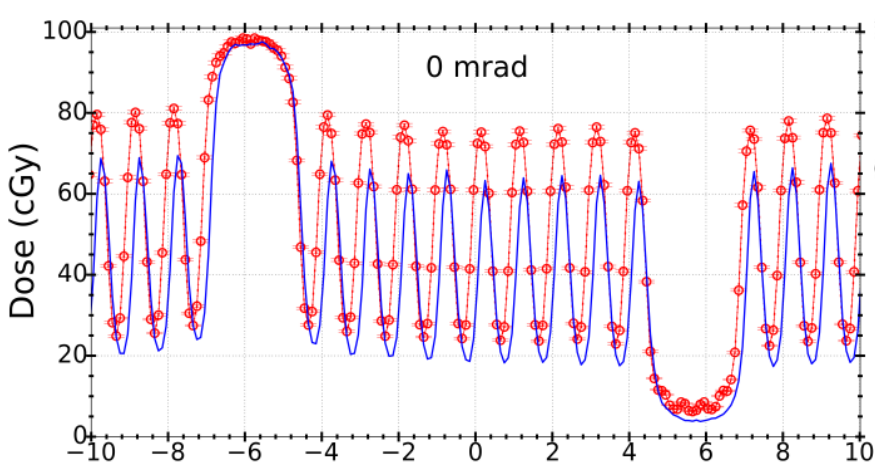

(a)

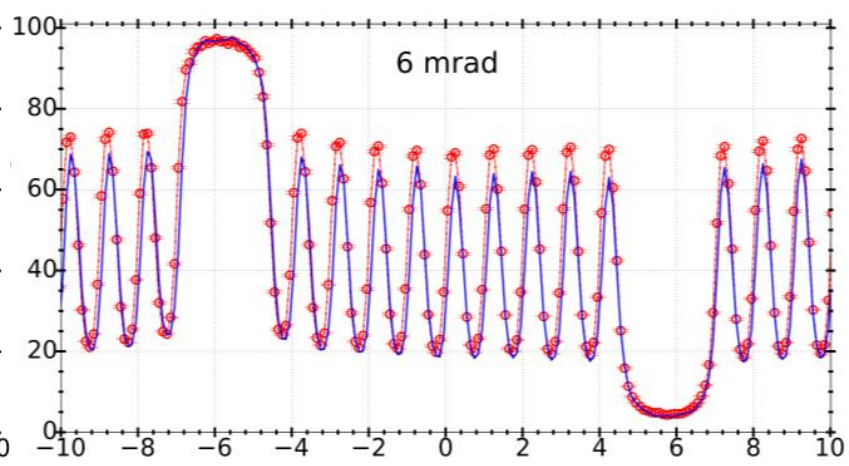

(b)

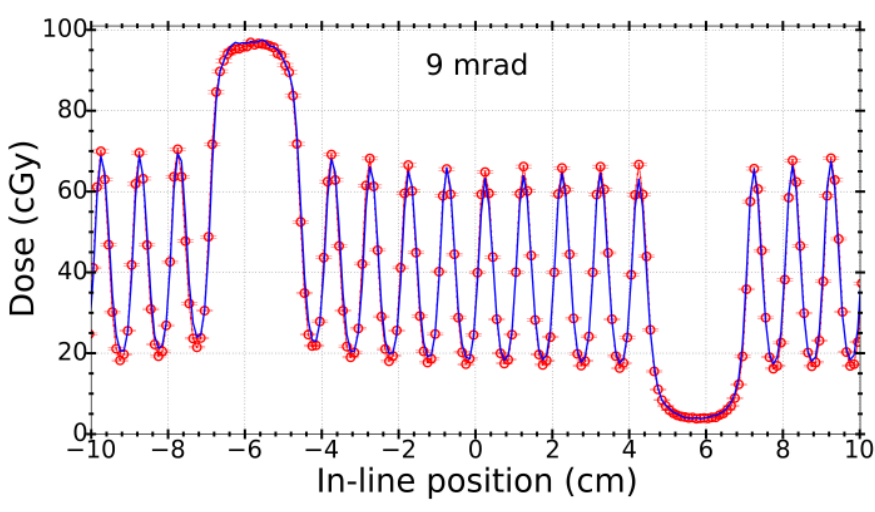

(c)

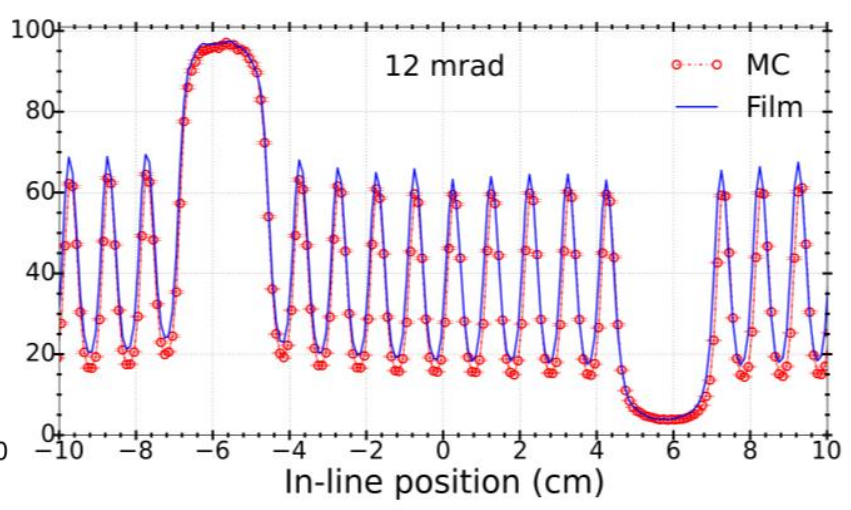

(d)

Figure 4.10 Comparison of dose profiles between film measurements (solid blue) and MC simulations (dotted red) for the field shown in Figure 4.4 for LBROT values of (a) 0 , (b) 6, (c) 9 and (d) 12 mrad. The best fit parameter was found to be LBROT $=9$ mrad.

Table 4.1 Mean percentage dose differences at the maxima and minima as well as average DTA (left and right) values at penumbra region from film measurements and MC simulations for different LBROT values. Uncertainties are statistical uncertainties associated with dose values at different maxima and minima from the profiles.

\begin{tabular}{cccc}
\hline \hline \multirow{2}{*}{ LBROT $(\mathrm{mrad})$} & \multicolumn{2}{c}{ Dose difference (MC/Film) $\%$} & \multirow{2}{*}{ Average DTA (mm) } \\
\cline { 2 - 3 } & Maxima (1-open leaf) & Minima (1-closed leaf) & \\
\hline 0 & $17.5 \pm 1.5$ & $31.7 \pm 3.0$ & 3.0 \\
6 & $8.7 \pm 1.3$ & $9.2 \pm 1.8$ & 1.0 \\
9 & $2.2 \pm 1.1$ & $-5.1 \pm 1.3$ & 0.2 \\
12 & $-7.1 \pm 1.0$ & $-14.1 \pm 1.0$ & 1.0 \\
\hline \hline
\end{tabular}


From the data presented in Figure 4.10 and Table 4.1 the impact of increasing leaf bank rotation on the isocenter dose profile can be seen. Also, it can be observed that the best agreement corresponds to MC simulations with LBROT value of $9 \mathrm{mrad}$. The resultant translations in the MLC leaf bank as derived from measurements and MC simulations and derived by suggested formulations were 0.42 and $0.41 \mathrm{~mm}$, respectively.

Using ion chamber measurements at the position of 5-open and 5-closed leaves, the average leaf transmission was measured to be $(4.3 \pm 0.1) \%$. From MC simulations, transmission was calculated to be $(4.1 \pm 0.1) \%$. It was observed that a decrease of $1.1 \%$ in the density of leaves (i.e. 18.7 to $18.5 \mathrm{~g} / \mathrm{cm}^{3}$ ) increases leaf transmission by $2.5 \%$. Impact of inter-leaf air gap on leaf transmission for LBRTO value of 9 mrad is shown in Fig 4.11 The nominal air gap was calculated to be $0.089 \mathrm{~mm}$.

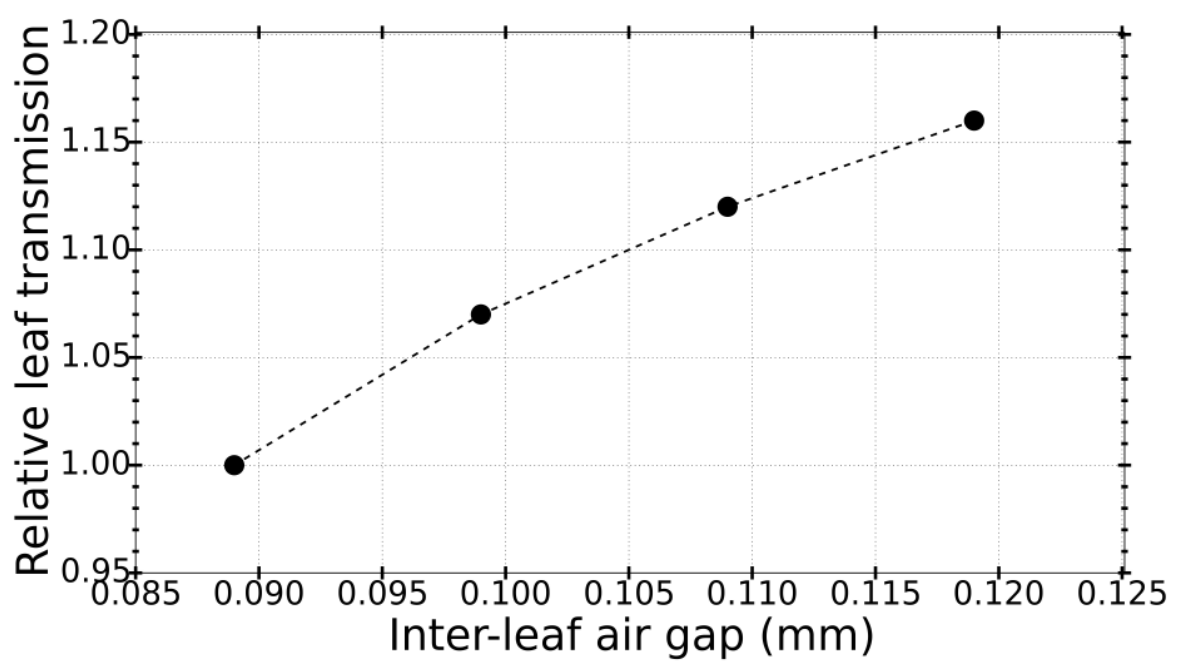

Figure 4.11 Variation of leaf transmission by increasing the inter-leaf air gap. All transmission values are normalized to the transmission corresponding to the nominal inter-leaf air gap. 
From this plot we can see that as the inter-leaf air gap increases by $0.001 \mathrm{~mm}$, the leaf transmission also increases by approximately $6.0 \%$. Parameters of the Elekta Infinity linac model that were derived based on the above analysis are shown in Table 4.2.

Table 4.2 Derived parameters of the Infinity linac model with their uncertainties.

\begin{tabular}{lc}
\hline \hline Parameter & Value \\
\hline Electron beam energy & $(6.6 \pm 0.1) \mathrm{MeV}$ \\
Beam width (cross-line) & $(2.1 \pm 0.1) \mathrm{mm}$ \\
Beam width (in-line) & $(1.0 \pm 0.1) \mathrm{mm}$ \\
Angular divergence & $(1.35 \pm 0.20) \mathrm{deg}$ \\
Leaf bank rotation angle (LBROT) & $(9.0 \pm 1.0) \mathrm{mrad}$ \\
Leaf material density & $18.5 \mathrm{~g} / \mathrm{cm}^{3}$ \\
\hline \hline
\end{tabular}

The Leaf composition (i.e. Tungsten alloy) was modified from the manufacturer provided values according to Table 4.3.

Table 4.3 The composition of the leaf as provided by manufacturer and adjusted in the MC beam model.

\begin{tabular}{lcc}
\hline \hline Material & \multicolumn{2}{c}{ Composition percentage } \\
\hline & Manufacturer & MC \\
Tungsten (W) & $95 \%$ & $96 \%$ \\
Nickel (Ni) & $3.75 \%$ & $3 \%$ \\
Iron (Fe) & $1.25 \%$ & $1 \%$ \\
& & \\
\hline \hline
\end{tabular}




\subsubsection{Analytic and virtual source model of the Elekta Infinity linac}

Figure 4.12 shows a comparison of photon fluence profiles at the isocenter plane from the analytic and Monte Carlo (BEAMnrc) linac models. Agreement of better than 1\% was observed at the position of maximum fluence. Also, the average DTA was found to be $0.04 \mathrm{~mm}$ at the penumbra region ( $50 \%$ of the maximum dose).

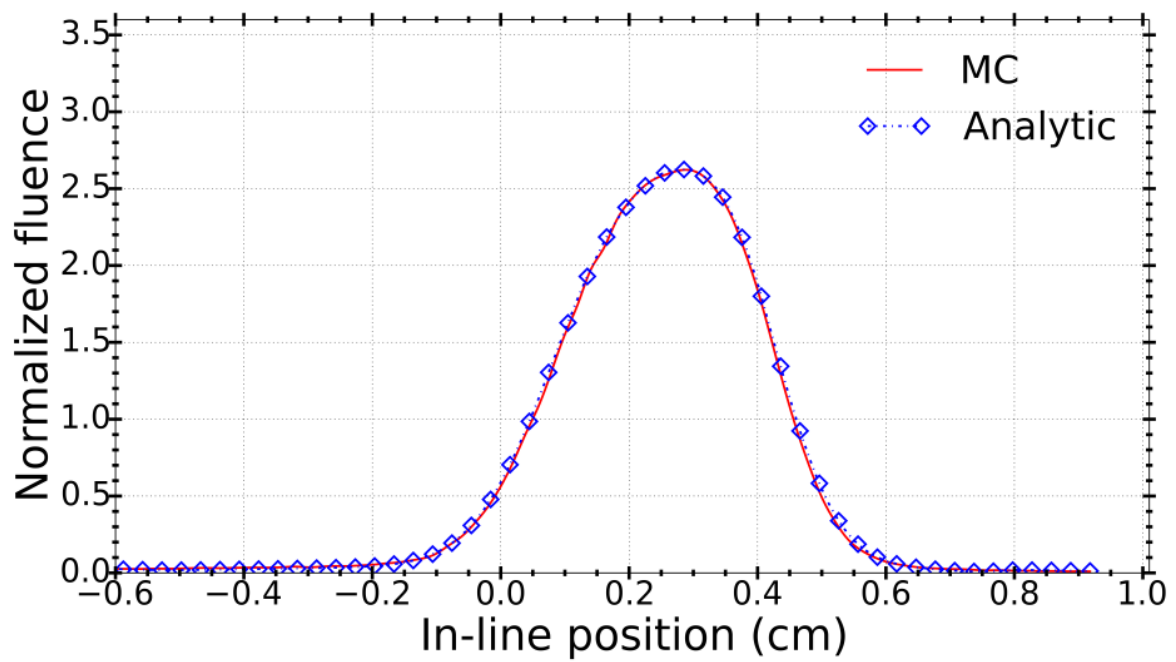

Figure 4.12 Photon fluence at the isocenter plane from MC simulations and analytic model calculations for $\mathrm{LBROT}=9 \mathrm{mrad}$. Fluence curves are normalized to their integral so that the integral of the resultant curve is equal to 1.

\subsubsection{Impact of analytic model parameters on the fluence at the isocenter plane}

The impact of modifying the analytic model parameters on the fluence at the isocenter plane, as described in Section 4.2.3, is illustrated in Figure 4.13. From Figure 4.13 (a) we can see that change in the maximum fluence due to increasing leaf bank rotation follows the same trend as in Figure 4.10 and Table 4.1. Due to the source occlusion, the maximum fluence drops from approximately $40 \%$ higher to $10 \%$ lower than the fluence at the nominal LBROT (9 mrad) as leaf bank rotation increases from 0 to $12 \mathrm{mrad}$. Ignoring leaf attenuation (100\% leaf transmission), as illustrated in Figure 4.13 (b), increases the 
maximum fluence by over $12 \%$ and average DTA by almost 4 times (up to $0.16 \mathrm{~mm}$ ) compared to the scenario where attenuation by the MLC leaves is considered. It can be observed from Figure 4.13 (c) that when the primary source is changed from a point source to a source with radius of $2 \mathrm{~mm}$ the maximum fluence decreases by approximately $25 \%$ due to source occlusion. Also, the average DTA worsens once the source size deviates from the nominal value of $1 \mathrm{~mm}$. 


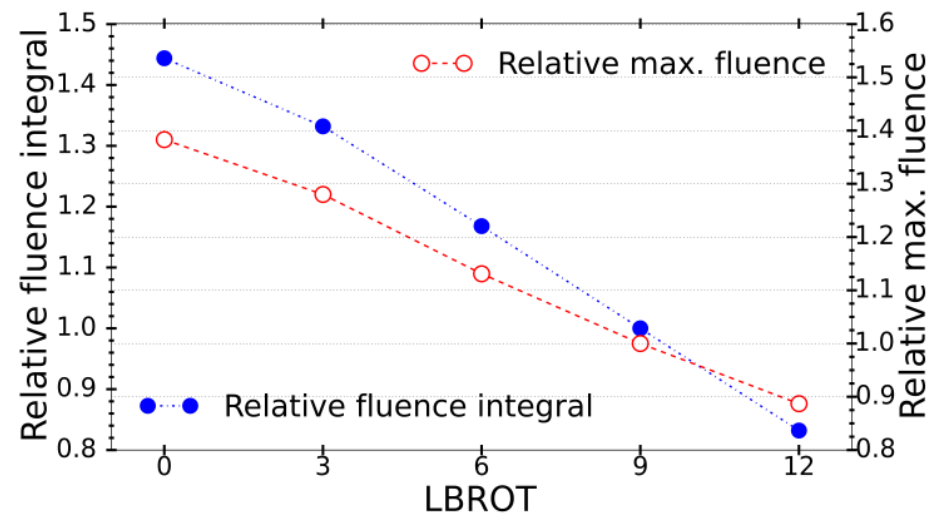

(a)

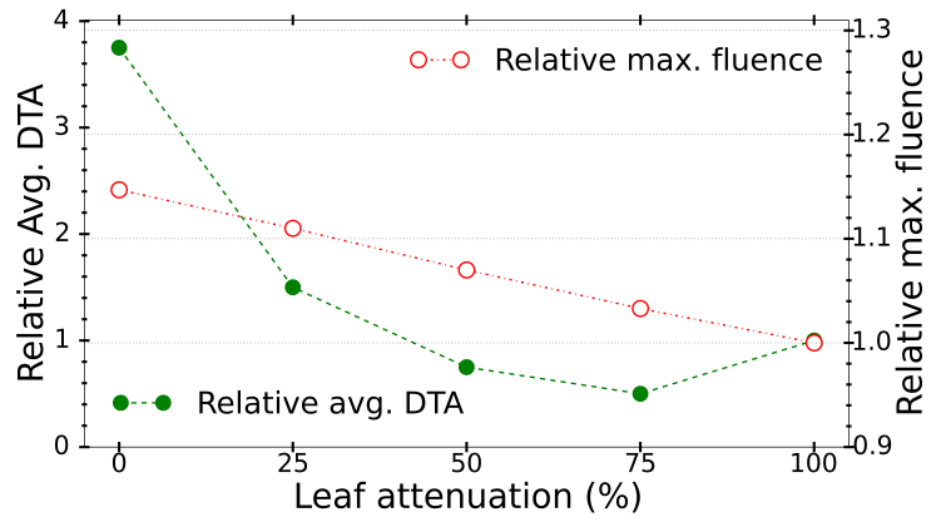

(b)

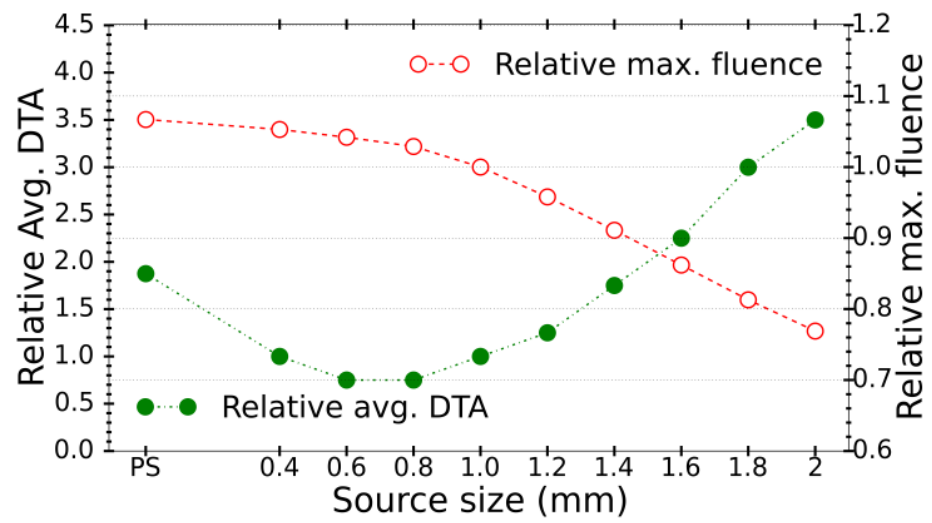

(c)

Figure 4.13 Impact of changes in (a) Leaf bank rotation (normalized to nominal value of leaf bank rotation or LBROT $=9 \mathrm{mrad})$, (b) Leaf attenuation (normalized to full MLC leaf attenuation) and (c) Primary source size (normalized to nominal source size of $1 \mathrm{~mm}$ ) on the relative fluence integral, maximum fluence and average DTA of the fluence in analytic model. 
Modeling the primary and secondary photon sources as mono-energetic sources with mean photon energies of 1.6 and $0.7 \mathrm{MV}$ instead of poly-energetic sources caused a negligible fluence change of approximately $0.1 \%$. However, a decrease of $0.2 \mathrm{MeV}$ in the mean energy of the primary photon source decreased the fluence integral by $1.1 \%$ due to the increase in the leaf attenuation. Using a uniform angular distribution as opposed to accurately modeling the angular distribution of the photons and its variation across the beam, decreased the fluence by approximately $3 \%$ and changed the DTA to $0.05 \mathrm{~mm}$. Modeling the tongue $\&$ groove in the MLC leaves caused the fluence to increase by over $2 \%$ and average DTA by $0.01 \mathrm{~mm}$. Exclusion of the secondary photon source reduced the fluence by less than $1 \%$ and caused almost no change in the average DTA. This result was expected as described previously in Section 4.2.3, since the secondary photon source models the photons due to head scatter and has very small contribution to the resultant photon fluence at the isocenter.

\subsubsection{Comparison of Monaco and EGSnre dose calculations}

Comparisons of dose profiles of the field shown in Figure 4.4 for Monaco calculations against film measurements and MC simulations using EGSnrc are shown in Figure 4.14 (a) and Figure 4.14 (b), respectively. Also, Table 4.4 shows percentage dose differences and DTA values as similarly reported in Table 4.1. 


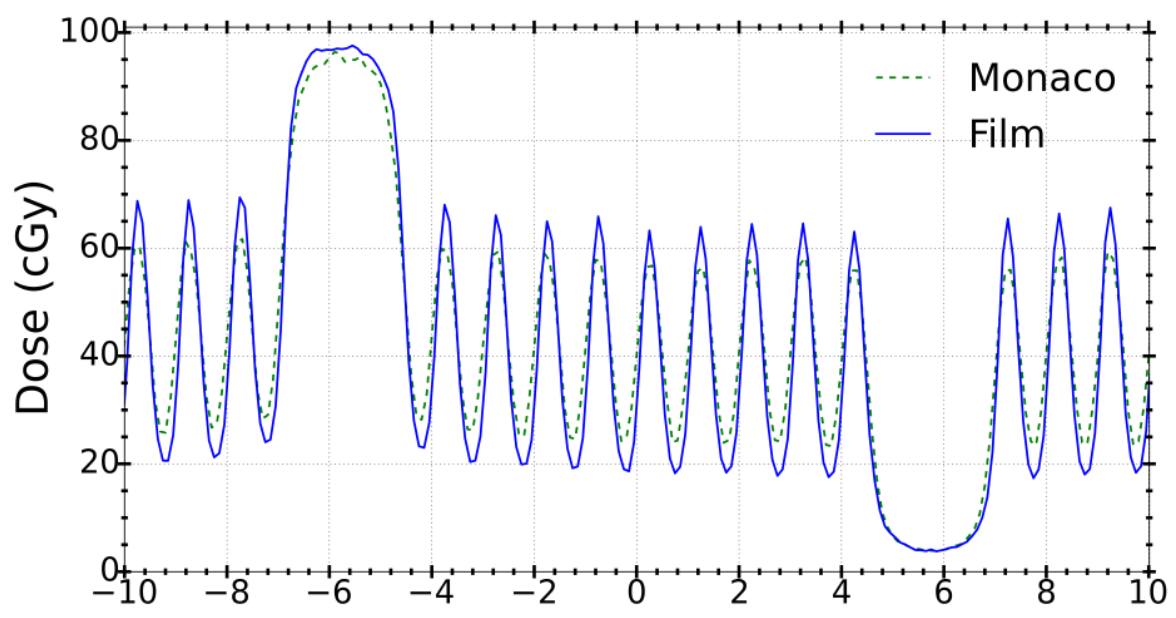

(a)

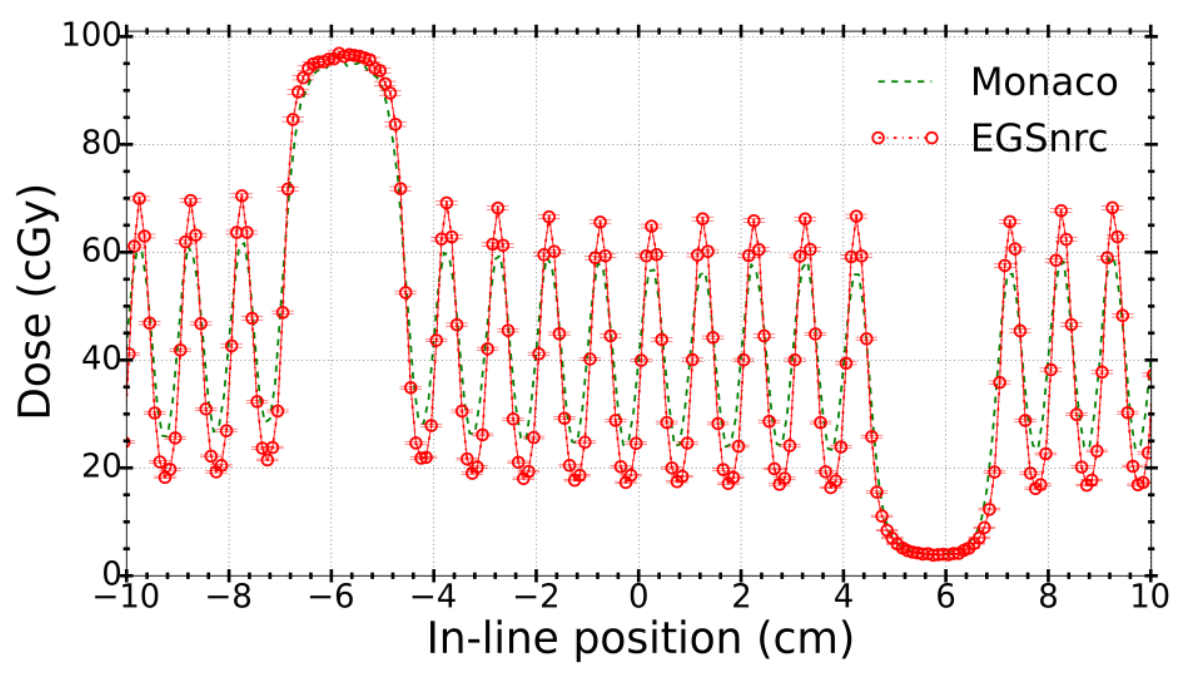

(b)

Figure 4.14 Comparison of Monaco calculations against (a) film measurements and (b) EGSnrc simulations for the field shown in Figure 4.4. 
Table 4.4 Mean percentage dose differences at the maxima and minima as well as average DTA (left and right) values at penumbra region from Monaco calculations against film measurements and EGSnrc simulations. Uncertainties are statistical uncertainties associated to dose values at different maxima and minima from the profiles.

\begin{tabular}{cccc}
\hline \hline \multirow{2}{*}{ LBROT (mrad) } & \multicolumn{2}{c}{ Dose difference \% } & \multirow{2}{*}{ Average DTA (mm) } \\
\cline { 2 - 3 } & Maxima (1-open leaf) & Minima (1-closed leaf) & \\
\hline \multirow{2}{*}{ Monaco/Film } & $-11.3 \pm 0.8$ & $33.7 \pm 2.9$ & 0.4 \\
Monaco/EGSnrc & $-13.2 \pm 1.0$ & $39.5 \pm 1.5$ & 0.5 \\
\hline \hline
\end{tabular}

To understand the observed differences, results of the analytic model from Sections 4.3.2 and 4.3.3 were used to modify the parameters in the BEAMnrc model of the linac to find a match between EGSnrc and Monaco calculations. First, the incident electron beam size (FWHM) along the in-line direction was changed from 1 to $2 \mathrm{~mm}$. Next, the MLC leaf geometry was modified to include the tongue \& groove as described in Section 4.2.4. The resultant dose profile from EGSnrc simulations is compared with Monaco calculations in Figure 4.15. It can be observed that implemented modifications in the MC model of the Elekta Infinity linac improved the agreement level between dose profiles from EGSnrc simulations and Monaco calculations. Table 4.5 shows the percentage dose differences and DTA values from EGSnrc simulations with modified beam width in the in-line position as well as inclusion of tongue \& groove in the MLC model. 


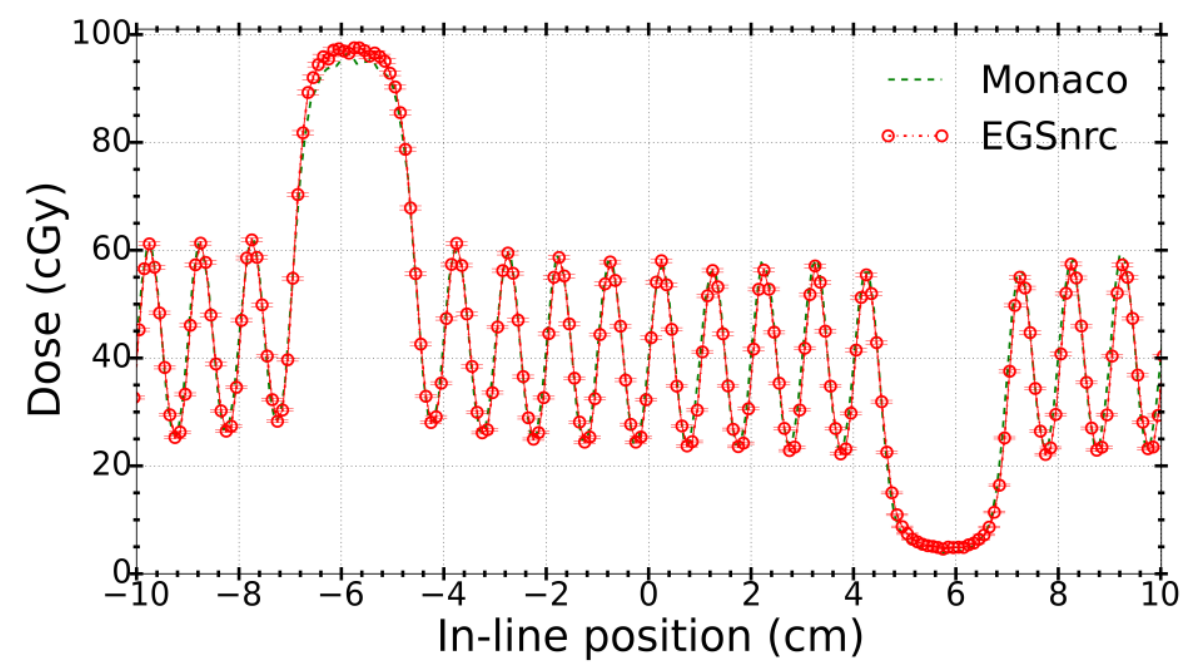

Figure 4.15 Comparison between Monaco calculations and EGSnrc simulations with modified parameters: $\mathrm{FWHM}=\mathbf{2} \mathbf{~ m m}$ in the in-line position and inclusion of tongue $\&$ groove. Dose differences were reduced to approximately $1 \%$ at the maxima and $2 \%$ at the minima.

Table 4.5 Effect of varying model parameters in EGSnrc simulations on mean percentage differences of dose at the maxima and minima, as well as average DTA (left and right) values at penumbra regions from Monaco calculations against EGSnrc simulations. Changes were made to increase the in-line beam width to $2 \mathrm{~mm}$ as well as including tongue \& groove to the MLC model. Uncertainties are statistical uncertainties associated with dose values at different maxima and minima from the profiles.

\begin{tabular}{|c|c|c|c|}
\hline \multirow{2}{*}{ Parameters modified } & \multicolumn{2}{|c|}{ Dose difference (Monaco/EGSnrc MC) \% } & \multirow{2}{*}{$\begin{array}{c}\text { Average DTA } \\
(\mathrm{mm})\end{array}$} \\
\hline & Maxima (1-open leaf) & Minima (1-closed leaf) & \\
\hline $\mathrm{FWHM}_{\text {In-line }}=2 \mathrm{~mm}$ & $2.7 \pm 1.0$ & $8.0 \pm 1.0$ & 0.3 \\
\hline $\begin{array}{l}\mathrm{FWHM}_{\text {In-line }}=2 \mathrm{~mm} \\
+ \text { tongue } \& \text { groove }\end{array}$ & $-0.4 \pm 0.5$ & $1.6 \pm 0.8$ & 0.2 \\
\hline
\end{tabular}




\subsection{Discussion}

To derive the mean energy and radial intensity (FWHM) of the incident electron beam of the MC model, PDD curves for a $5 \times 5 \mathrm{~cm}^{2}$ field as well as OAR (i.e. cross- and inline profile) measurements of different field sizes were used, respectively. One approach, followed by Almberg et al. ${ }^{96}$, is to take advantage of the energy independent response of the film and use film measurements of the penumbra and build-up regions. In this work similar measurements were performed using diodes combined with ion chamber to complement diode measurements and to account for the energy dependence of the diodes in large field sizes. Further adjustment of the FWHM of the radial intensity profile was performed using output factor measurements. The RDFs of small fields (e.g. $\left.2 \times 2 \mathrm{~cm}^{2}\right)$ were measured using small volume ion chamber and photon diodes. The angular distribution of the electron beam was determined from profile measurements of large field sizes. A very good agreement was found between MC calculated and measured curves for all PDD, profiles and output factor measurements. A passing rate of $100 \%$ was observed when comparing simulated PDD curves against measurements using a 1\%/1 mm gamma criteria. As for cross- and in-line profiles, all simulated dose points passed a $2 \% / 1 \mathrm{~mm}$ gamma comparison against measurements for field sizes smaller than or equal to $20 \times 20 \mathrm{~cm}^{2}$. The passing rate for larger field sizes was better than 95\%. For RDFs, worst agreement was less than $0.5 \%$ for the $40 \times 40 \mathrm{~cm}^{2}$ field size.

The beam modulation system of the Infinity linac is the Agility MLC comprising 160 leaves with projected leaf width of $5 \mathrm{~mm}$ at the isocenter. The choice of an alternating open-closed leaves field to derive the leaf bank rotation parameter (LBROT), enabled us to apply a small field size and find the optimal value of the leaf bank rotation. An LBROT 
value of $9 \mathrm{mrad}$ was found to give the smallest dose differences (at maxima and minima) and DTA values between MC simulations and film measurements. The optimal inter-leaf air gap for this leaf geometry was found to be almost $0.09 \mathrm{~mm}$. The average measured and calculated leaf transmissions were found to agree within $5 \%$ of each other. Occasionally, appropriate corrections to vendor provided information about the material and density of different components of the linac head are required.$^{89}$ In this study, the composition and density of the leaves, which are made of a tungsten alloy, were adjusted in the MC model for better agreement with measurements. The fitted values of the MLC leaf composition were within $1 \%$ of the values provided by the manufacturer and density was higher by approximately $3 \%$.

The virtual source model, adopted to analytically calculate the photon fluence at the isocenter plane from a small field, comprises two photon sources. The electron source was not included because the contribution of contamination electrons to the resultant fluence at the isocenter plane was less than $1 \%$. The model used a simple ray tracing and exponential attenuation relationship to model the impact of the MLC leaves. Despite these approximations, our analytic model provided a simple, quick and reliable method to investigate the sensitivity of the fluence to linac model parameters and to explain the disagreements between film measurements and Monaco calculations. For example, it was observed that the fluence at the isocenter plane is highly sensitive to the size of the primary photon source. Also, the integral fluence was shown to be quite sensitive to the change in the mean energy of the primary source. On the other hand, the fluence was minimally sensitive to using mean energy values for the photon sources rather than a spatial energy distribution. These results are in agreement with findings from groups who studied 
sensitivity of the MC model parameters to the characteristics of the incident electron beam..$^{92,93,95,97,99-101}$ Due to the fact that the secondary photon source only represents the scattered photons, the fluence showed negligible sensitivity to excluding this source or changing its parameters (e.g. mean energy) in the presence of a small field. However, the contribution of the secondary photon source could become more important for larger field sizes.

Regarding the impact of the leaf bank rotation, the change in the calculated fluence follows the same trend as the dose differences at the maxima in the dose profile of the alternating field in both beam models. Increasing the leaf bank rotation causes a decrease in the fluence due to increased occlusion of the source. Inappropriate modeling of the leaf transmission (e.g. leaf density, attenuation coefficient and leaf thickness) can also affect the fluence at the isocenter plane. However, the sensitivity of the fluence to this parameter was not found to be large since a $25 \%$ decrease in the leaf attenuation causes $4 \%$ error in the fluence. Thus, we can see that although it is important to properly model the transmission of the leaves, error of a few percent in transmission parameters is not an important source of error in the fluence calculated at the isocenter plane. This result was similar to the findings from MC simulations with modified leaf density, as explained earlier. In the analytic model, the path length of the rays traversing the MLC leaves was defined according to their sampled trajectory. A change of less than $0.1 \%$ in the fluence was observed if the rays were assumed to travel exclusively along the direction parallel to the beam axis (as is used in Monaco beam model) rather than the accurate oblique path. This was predictable considering small trajectory angles of the bremsstrahlung photons of 
the target (i.e. primary photon source) and small contribution of the scatter photons from the primary collimator and flattening filter (i.e. secondary source).

Results of the analytic model calculations confirmed the findings shown by Sikora et al. ${ }^{108}$ on the importance of accurate modeling of the primary photon source size for the small fields. The change in the size of the primary photon source improved the agreement between Monaco and EGSnrc calculated doses by over 10 and 30\% at maxima and minima, respectively. Another important parameter in photon fluence calculations for small field sizes is the modeling of the multi-leaf collimator. According to the MLC geometry parameters from the Monaco beam model, in addition to the leaf bank rotation, a tongue \& groove design with a groove width of $0.4 \mathrm{~mm}$ was implemented in the MLC model. To study the impact of this tongue \& groove design, the leaf geometry in our BEAMnrc model was modified using the tongue $\&$ groove width specifications in the MLC geometry file in Monaco. It was found that addition of tongue \& groove to the current model of MLC leaves in the BEAMnrc model improved the agreement at both maxima and minima by about 3 and $6 \%$, respectively, in addition to the initial change in the source size (i.e. using a source size with FWHM $=2 \mathrm{~mm}$ ). This is due to the insertion of groove and as a result less occlusion of the source and higher number of photons reaching the isocenter. Similar results were observed when using a leaf bank rotation of $8 \mathrm{mrad}$ (no added tongue \& groove) rather than 9 mrad which results in less source occlusion as well.

From these findings we can conclude that the observed disagreements between Monaco and measured/EGSnrc calculated dose profiles, shown in Figure 4.14, could be associated mainly to the size of the primary source as modeled in the beam model implemented in Monaco. Comparison of EGSnrc simulations using the same focal spot 
size as in Monaco (i.e. FWHM $=2 \mathrm{~mm}$ ) against Monaco calculations (Figure 4.15) confirmed this conclusion as the dose calculated by EGSnrc dropped by approximately $16 \%$ at the maxima or position of 1 -open leaf. Also, since the entire source cannot be viewed from the center of the field at the isocenter (i.e. source occlusion), the penumbra region widens, which results in the reduction of the DTA between EGSnrc and Monaco calculated dose profiles and therefore better agreement. These findings are a quantitative description of the impact of source occlusion as explained in Section 4.1.4 and illustrated in Figure 4.1. The superposition of widened penumbrae from adjacent open leaves results in a $30 \%$ dose increase at the position of 1-closed leaf or minima of the dose profile. Overall, changing the source size from 1 to $2 \mathrm{~mm}$ and insertion of tongue $\&$ groove changed the result of EGSnrc simulations to be closer to the calculations obtained from Monaco.

\subsection{Conclusions}

As simple as creating a Monte Carlo model of a linear accelerator head could be, determining appropriate model parameters plays an important role in accurate dose calculations. The same principle applies when using a virtual source model to analytically calculate the photon fluence resultant from a treatment head. Moreover, with advancements in radiation delivery techniques, using small fields has become inevitable in radiotherapy. This introduces more complexity to fine tuning model parameters of MC or analytic source models (i.e. beam and collimation parameters) due to the challenges associated with small fields.

This work presents a detailed MC model of a $6 \mathrm{MV}$ Elekta Infinity linac with Agility MLC that was validated against measurements. Also, the possibility of using a simple analytic model as a quick method to study the sensitivity of different model 
parameters of a linac when delivering treatments with small field sizes was demonstrated. One important result that was studied using the analytic model and confirmed by MC simulations and measurements was the importance of adjustment of primary photon source size for small fields. Another important result obtained in this study was that modeling of the MLC leaf design (i.e. leaf bank rotation and/or tongue \& groove) is essential for accurate simulation of delivered dose (maximum dose and penumbrae). Results from this study helped us explain the discrepancies observed between dose calculations obtained from the Monaco treatment planning system and film measurements/EGSnrc simulations of the Elekta Infinity linac.

In conclusion, simulation of advanced techniques such as IMRT, VMAT and SBRT that comprise small fields requires a realistic MLC model as well as adjusted size of the primary photon source in the VSM used in treatment planning systems. Results of this study could be valuable to cancer centers that use the Elekta Infinity linac to help ensure accurate dose calculations for the above-mentioned treatment techniques. 


\section{Chapter 5}

\section{Phantoms}

\subsection{Introduction}

While 4D dose calculation algorithms account for both translations and deformations of the anatomy due to breathing ${ }^{60,68,82}$, verification of such algorithms requires phantoms that simulate the motion and deformation of different organ (e.g. lung, liver, etc.) tissues as well as the tumor. Another design consideration for such phantoms is their capability to accommodate different dosimeters (e.g. film, ion chamber, MOSFET) so they can be used for dosimetric verification of the treatment delivery as well as validation of 4D dose calculation algorithms. Reproducibility of the motion as well as the phantom geometry from one setup to the next is another important characteristic required of the phantoms used for 4D dosimetry/dose calculation purposes. Several rigid "breathing" phantoms are commercially available, including the Quasar respiratory motion phantom (Modus Medical, London, ON, Canada), Dynamic thorax phantom (CIRS, Norfolk, VA, USA) and Respiratory gating platform (Standard Imaging Inc., Middleton, WI, USA). In addition to these commercial models, several research phantoms using rigid motion have also been developed over the past years ${ }^{134-137}$. However, these phantoms, although suitable for dosimetric measurements, are moving in a rigid manner and therefore simulate only the anatomy translations during respiration.

Some research groups have developed tissue-equivalent phantoms that are capable

of simulating motions and deformations induced by respiration. Kashani et al. ${ }^{138}$ used a thoracic phantom as the exterior shell of a deformable lung phantom with an iodine-infused foam as lung. They inserted in the foam, rigid tumors made from different materials and 
compressed/decompressed the foam using a programmable actuator system. The phantom was used as a validation tool for image registration algorithms ${ }^{139}$. Recently, Cheung et $a l^{140}$ constructed an externally and internally deformable phantom and tested its geometrical accuracy and reproducibility under sinusoidal and irregular motion patterns. They used the outer shell of a commercially available lung phantom with flexible anterior surface and filled it with a block of latex foam which was deformed using a programmable platform. Other groups ${ }^{141,142}$ developed and validated real tissue phantoms. Szegedi et al. ${ }^{141}$ placed porcine liver between two rigid supporting membranes and induced the respiratory motion by pulling and pushing one membrane while the other was fixed. To evaluate performance of $4 \mathrm{D}$ magnetic resonance imaging (MRI), Remmert et al. ${ }^{142}$ developed a dynamic phantom based on fresh porcine heart and lung organs placed in a chest wall made of a double-walled container filled with saline solution. The inflations and deflations of a water-filled balloon simulated the diaphragm while a vacuum pump connected to the container allowed expansion of the lung. Some recently developed phantoms ${ }^{143-145}$ used silicon or latex balloons filled with dampened sponge to simulate the lung with ${ }^{144,145}$ or without ${ }^{143}$ moving internal targets inside cylindrical bodies. One disadvantage of phantoms made of dampened sponge is the lack of reproducible properties due to the need to uniformly dampen the sponge before every measurement in order to obtain tissue-like attenuation properties. Also, design of the previously mentioned phantoms did not allow dosimetric evaluations and they were mainly validated geometrically.

Other groups designed phantoms for dosimetric validation purposes. Nioutsikou et $a l .{ }^{146}$ built a breathing phantom composed of two accordion-type flexible bottles mounted 
inside a container to simulate lungs. They also used dampened sponge to fill the lungs and placed a solid cylindrical tumor with several cuts to hold film in one of the lungs. The phantom was used to quantify the impact of respiratory motion on the dose delivered to the tumor during static conformal and IMRT plan deliveries. The phantom designed by Serban et al. ${ }^{147}$ used a Lucite cylinder as external body filled with water and a latex balloon stuffed with dampened natural sponge to simulate lung. A deformable Dermasol tumor was incised (to hold film and micro-MOSFET) and embedded in the sponge along with nylon wires and Lucite beads to emulate lung structures. Cherpak et al. ${ }^{148}$ used this phantom with the RADPOS 4D dosimetry system to verify the tumor dose and motion in a deformable phantom. Court et al. ${ }^{149}$ created a flexible resin tumor based on computed tomography (CT) images of an actual tumor and placed it inside an anthropomorphic breathing phantom. They inserted twenty micro-MOSFETs inside the tumor to measure the effect of real-patient respiratory motion on the tumor dose during conformal, IMRT and VMAT beam deliveries. Recently, Mann et al. ${ }^{150}$ developed a phantom made of porcine lung placed inside a PMMA cylindrical shell and inserted a 3D polymer gel dosimetry tumor at the center of the phantom. Evaluation of the phantom was performed by comparing measured and planned dose distributions from stationary and moving states of the phantom during static conformal beam deliveries. Vinogradskiy et al. ${ }^{151}$ designed a deformable phantom made from slices of sponge and foam placed inside a cylinder and compressed/decompressed it with a piston attached to a programmable actuator. A cylindrical rigid target which contained a space for film in the sagittal and coronal planes was embedded in one of the sponges. Also, three films in plastic cassettes were sandwiched between the sponges in the transverse plane. 
This chapter presents the phantoms we utilized to simulate respiratory-induced tumor motion to achieve the goal of this work which is experimental verifications of 4DMC simulations. We introduce the Quasar phantom which simulates rigid translations and was used for preliminary measurements and validations in this work. A deformable phantom was then used for more realistic situations and verifications. This chapter includes the design and motion assessment of our recently developed deformable phantom with characteristics similar to lung, containing a tissue-like elastic tumor. The latest modifications to the phantom enable simulations of sinusoidal and irregular respiratory motion traces. Moreover, design of the phantom allows a reproducible setup through easy assembly of the phantom components. In addition, film and RADPOS can be inserted and removed easily during assembly and thus measurement of point dose and dose distribution is enabled. Placing RADPOS inside the phantom allows also verification of the internal motion in real-time and enables obtaining the motion trace for 4DMC simulations. This motion can be also correlated to the motion of the diaphragm during one or several respiratory phases.

\subsection{Materials and Methods}

\subsubsection{Quasar respiratory motion phantom}

The Quasar respiratory motion phantom, as shown in Figure 5.1 (a), is made of an oval-shaped acrylic body that holds a cylindrical lung insert. The lung insert is made of cedar wood and splits in two halves (Figure 5.1 (b)) to allow insertion of film. A $3 \mathrm{~cm}$ diameter Solid Water sphere, shown in Figure 5.1 (c), is positioned at the center of the insert to mimic the tumor. To allow for RADPOS probe insertions, a special groove was cut into the tumor as well as in the lung insert. The Quasar phantom simulates the breathing 
motion by allowing the lung insert to move rigidly in the S-I direction according to a sinusoidal or programmed respiratory motion pattern with a user-defined frequency (sinusoidal motion) and amplitude. In this work, a single reproducible sinusoidal motion pattern with a peak-to-peak $(\mathrm{P}-\mathrm{P})$ respiratory amplitude of $3.6 \mathrm{~cm}$ and $3.3 \mathrm{~s}$ period was used.
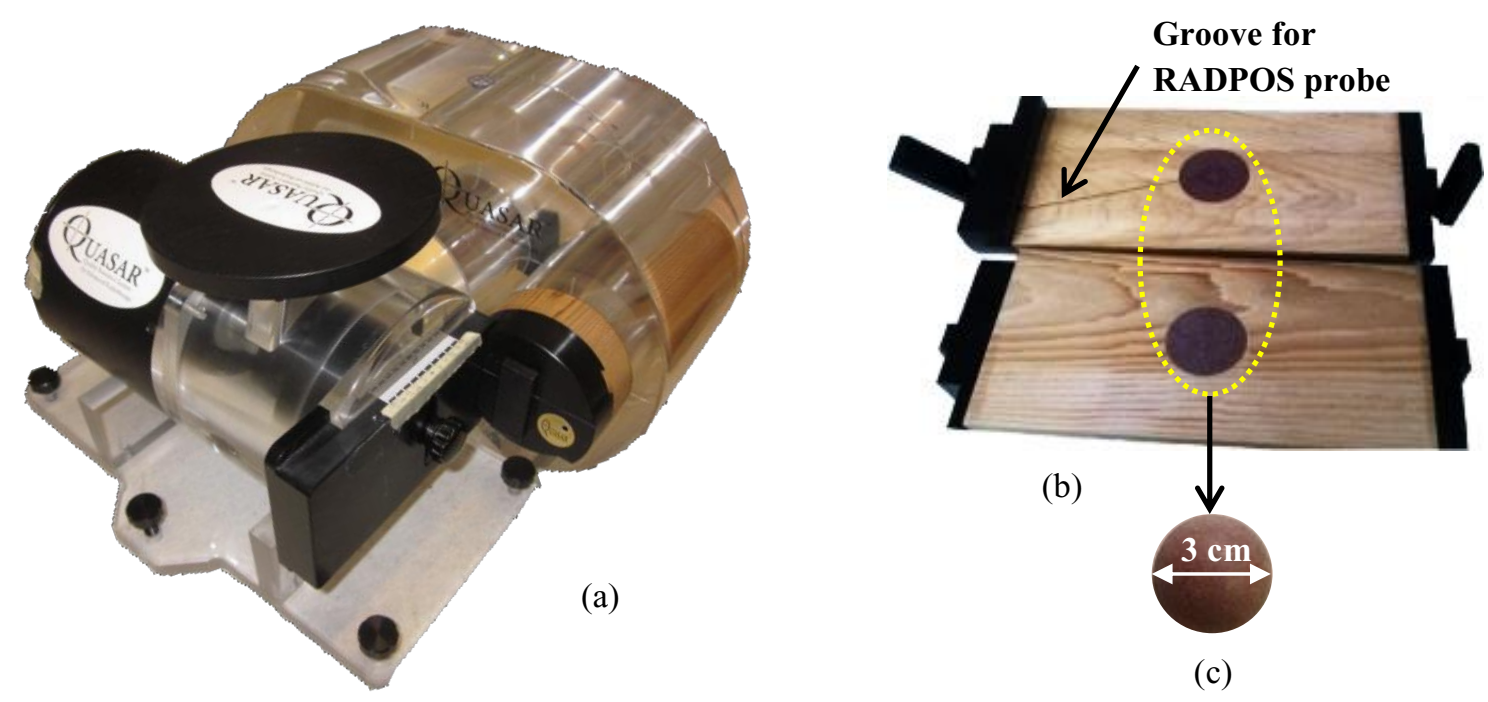

(c)

Figure 5.1 (a) The Quasar respiratory motion phantom, (b) wooden lung insert in two halves with the groove to insert RADPOS probe and (c) Solid Water tumor.

\subsubsection{Deformable lung phantom}

A deformable lung phantom, shown in Figure 5.2 (a), was developed based on modifications to the design previously proposed by Serban et al. ${ }^{147}$. To simulate the lung, flexible foam with density of $0.17 \pm 0.02 \mathrm{~g} / \mathrm{cm}^{3}$ was molded into a cylindrical insert with $18 \mathrm{~cm}$ length and $13 \mathrm{~cm}$ diameter. The insert was placed inside an external cylindrical body made of Lucite with dimensions equal to $20 \mathrm{~cm}$ in length and $14 \mathrm{~cm}$ in diameter. The foam composition was selected to have similar CT numbers (-830 \pm 37$) \mathrm{HU}$ (Hounsfield Units) to human lung tissue. A plug (Figure $5.2(\mathrm{~b})$ ) of $11 \mathrm{~cm}$ in length and $4 \mathrm{~cm}$ in diameter was molded from the same foam material and was fixed into a cylindrical hollow inside the 
lung insert. The plug was used to hold a $2.6 \mathrm{~cm}$ diameter tumor made of silicon rubber with density of $1.05 \pm 0.04 \mathrm{~g} / \mathrm{cm}^{3}$ (Figure $5.2(\mathrm{c})$ ). Similar to the lung insert of the Quasar phantom, a special groove was molded inside the tumor and plug as well as its outer surface (top and bottom) to place RADPOS probes. Moreover, film can be placed inside the plug to measure dose distribution. 32 Lucite beads, with radius of $1.6 \mathrm{~mm}$, were injected as landmarks throughout the phantom to help with the deformable image registration and quantifying the phantom motion (Figure 5.2 (d)). At the inferior end of the phantom, a piston attached to a DC motor simulated the diaphragm and the breathing motion by compressing and decompressing the lung phantom in the S-I direction, according to a sinusoidal motion with $4 \mathrm{~cm}$ P-P respiratory motion with varying motion periods. For this work the phantom oscillated at a period of $3.3 \mathrm{~s}$.

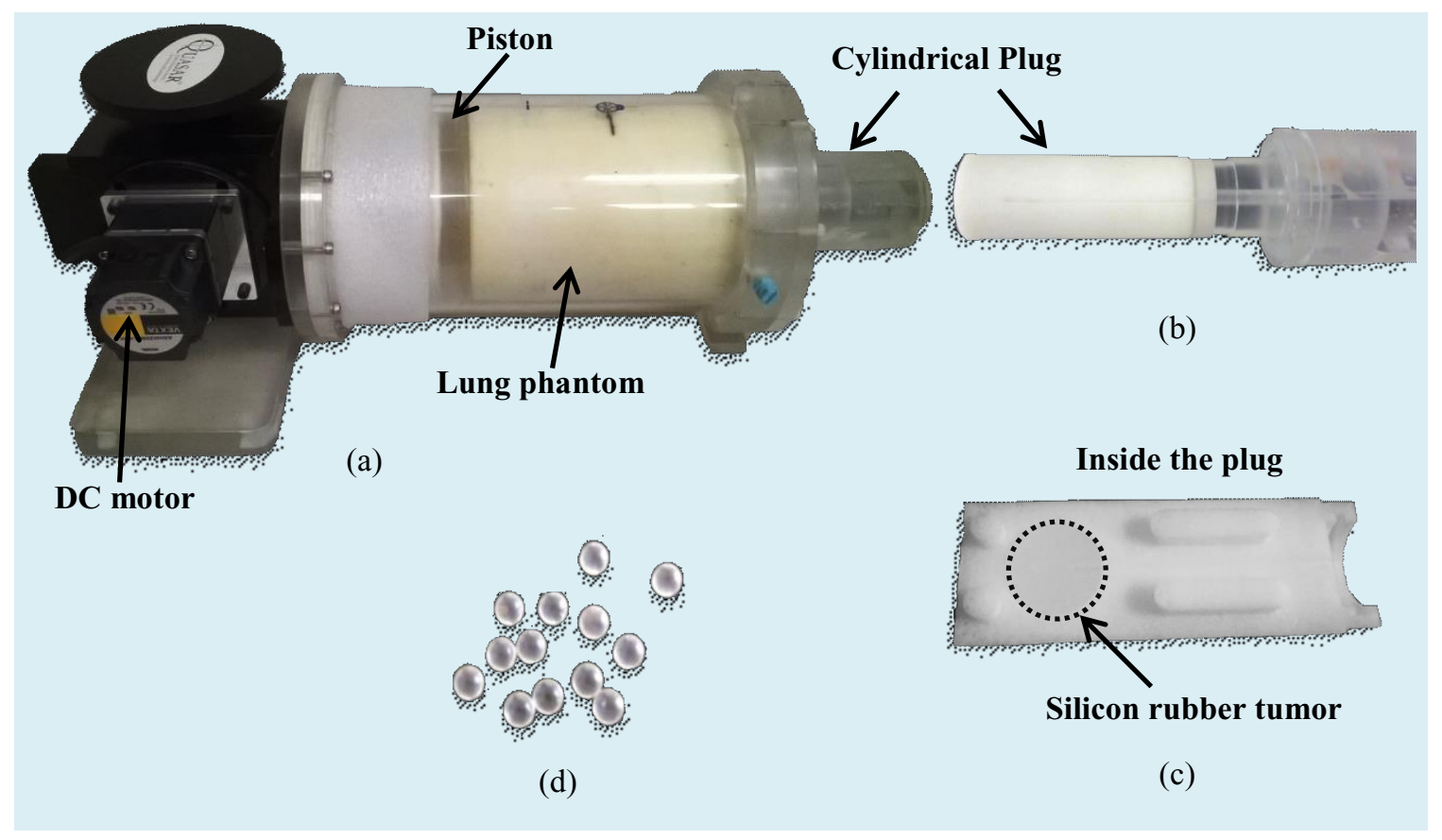

Figure 5.2 (a) The deformable lung phantom with different parts including the flexible foam, plug, piston and DC motor, (b) Cylindrical plug fixes inside the foam, (c) silicon rubber tumor inside the plug and (d) Lucite beads injected throughout the phantom. 


\subsubsection{Motion assessment and reproducibility}

To assess the motion reproducibility of the phantom in 3D (S-I, L-R and A-P), the RADPOS system was used. RADPOS detectors were placed at the tumor center (inside the plug, approximately $9 \mathrm{~cm}$ from piston) as well as on the top and bottom surfaces of the plug (Figure 5.3). The RADPOS on the bottom surface of the plug was aligned with the one inside the tumor while the top surface RADPOS was offset approximately $1 \mathrm{~cm}$ from the tumor center along the S-I axis.

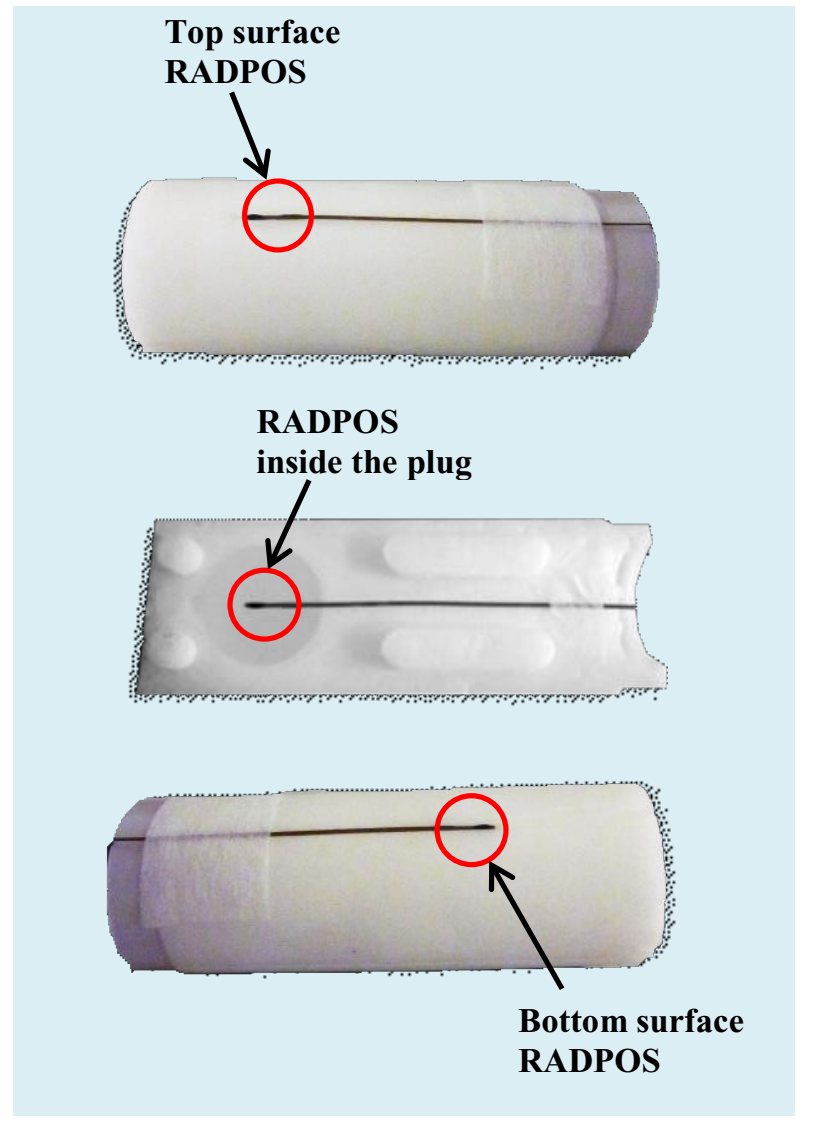

Figure 5.3 RADPOS detectors on the top surface (top), inside (middle) and bottom surface (bottom) of the plug.

Motion measurements were repeated 10 times independently (turning the motor on/off) to evaluate the motion reproducibility of the phantom. Displacement magnitude for 
these points in the S-I direction was also measured on the 4DCT scans of the phantom using End-of-Inhale (EOI) and End-of-Exhale (EOE) images.

\subsubsection{Deformable programmable lung phantom}

The phantom presented in Section 5.2.2 was further modified to simulate realistic respiratory motion profiles by replacing the DC motor with a programmable servo motor (Figure 5.4 (top)). A Scotch Yoke mechanism was used to transform the rotational motion of the motor to linear motion at the piston end as shown in Figure 5.4 (bottom).

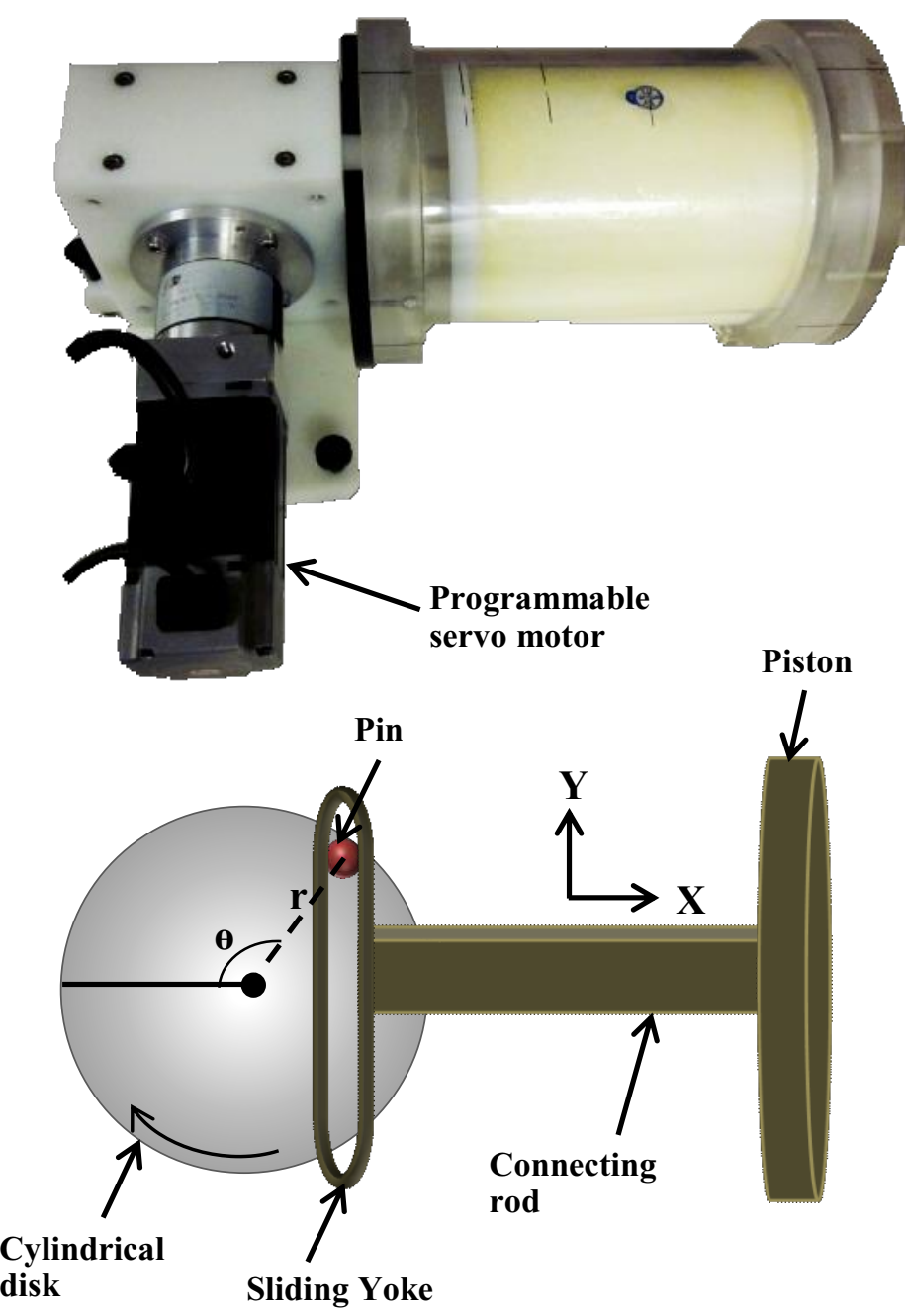

Figure 5.4 Deformable lung phantom with programmable servo motor to simulate realistic respiratory motion profiles (top) and Scotch Yoke mechanism to transform the rotational motion of the motor to linear motion at the piston (bottom). 
A cylindrical disk with radius $r$ is attached to the motor disk. Rotation of the motor causes the pin at the edge of the disk to slide in the vertical direction $(\mathrm{Y})$ inside the sliding yoke. This vertical motion results in the horizontal motion of the connecting rod and as a result the piston. The magnitude of the linear motion (X), is related to $\mathrm{r}$ and $\theta$, which are radius and angle of rotation, respectively. This linear mtion can be calculated by equation 5.1:

$$
X=r(1-\cos \theta)
$$

where $\mathrm{r}=2.5 \mathrm{~cm}$ and $0^{\circ} \leq \theta \leq 180^{\circ}$ for the phantom used in this work. The maximum achievable peak-to-peak (P-P) amplitude at the piston is $5 \mathrm{~cm}$. Also, the maximum breathing frequency of the phantom is $4 \mathrm{~Hz}$ which correlates to 4 breaths per second or a breathing period of $0.25 \mathrm{~s}$.

\subsubsection{Motion assessment and reproducibility}

The phantom diaphragm was driven with sinusoidal and irregular motion profiles and the realized motion was recorded with a temporal resolution of $100 \mathrm{~ms}$ by 3 RADPOS detectors placed at the center of the tumor as well as on the top and bottom surfaces of the plug. Moreover, motion of the diaphragm was recorded by the motion controller used to program the servo motor, to be compared against the motion trace recorded by RADPOS. This methodology enabled us to assess the reproducibility of the motion of the phantom (i.e. motor motion and foam). Also, by disassembling and reassembling the RADPOS detectors and plug, we were able to evaluate the reproducibility of the phantom setup from one assembly to another.

Sinusoidal motion profiles were simulated at P-P diaphragm amplitudes of 1, 1.5, 2, 2.5 and $3 \mathrm{~cm}$ and for periods of $2-7 \mathrm{~s}$ in steps of $1 \mathrm{~s}$. Measurements were repeated 6 
times over 3 days (i.e. 2 datasets per day) to evaluate both inter- and intra-day reproducibility and variations. To evaluate intra-day reproducibility (i.e. phantom setup reproducibility), the plug and RADPOS detectors were disassembled and reassembled. Also, the order in which motion profiles were tested was chosen randomly to eliminate the possibility of a pattern or systematic error occurring during recordings.

For the irregular motion profiles, 16 different sample patient profiles at P-P diaphragm amplitudes of $1,1.5,2,2.5$ and $3 \mathrm{~cm}$ were tested to fully evaluate the performance of the phantom. Measurements were repeated 5 times for each motion profile over 5 days (i.e. 1 dataset per day). Because the average duration of the profiles was over $240 \mathrm{~s}$, it was not practical to repeat measurements in one day. It is valid to assume that intra-day variations were similar to the values measured for sinusoidal motion profiles. A Python code, which detected the peaks and valleys of the recorded motion traces, was used to find the average P-P amplitudes and their variations for irregular motion patterns. This was necessary due to variations in the P-P amplitudes of such profiles compared to sinusoidal motion patterns.

\subsection{Results}

\subsubsection{Deformable lung phantom with DC motor}

The density and volume of lung insert were found to increase by $11.5 \%$ and decrease by $28.7 \%$, respectively, from EOI to EOE. Various levels of hysteresis (Figure 5.5) were observed based on trajectories of selected landmarks (Lucite beads). Larger hysteresis was observed for points closer to the piston and smaller in points further away. This shows that the lung insert follows slightly different deformation paths between compression and decompression. 

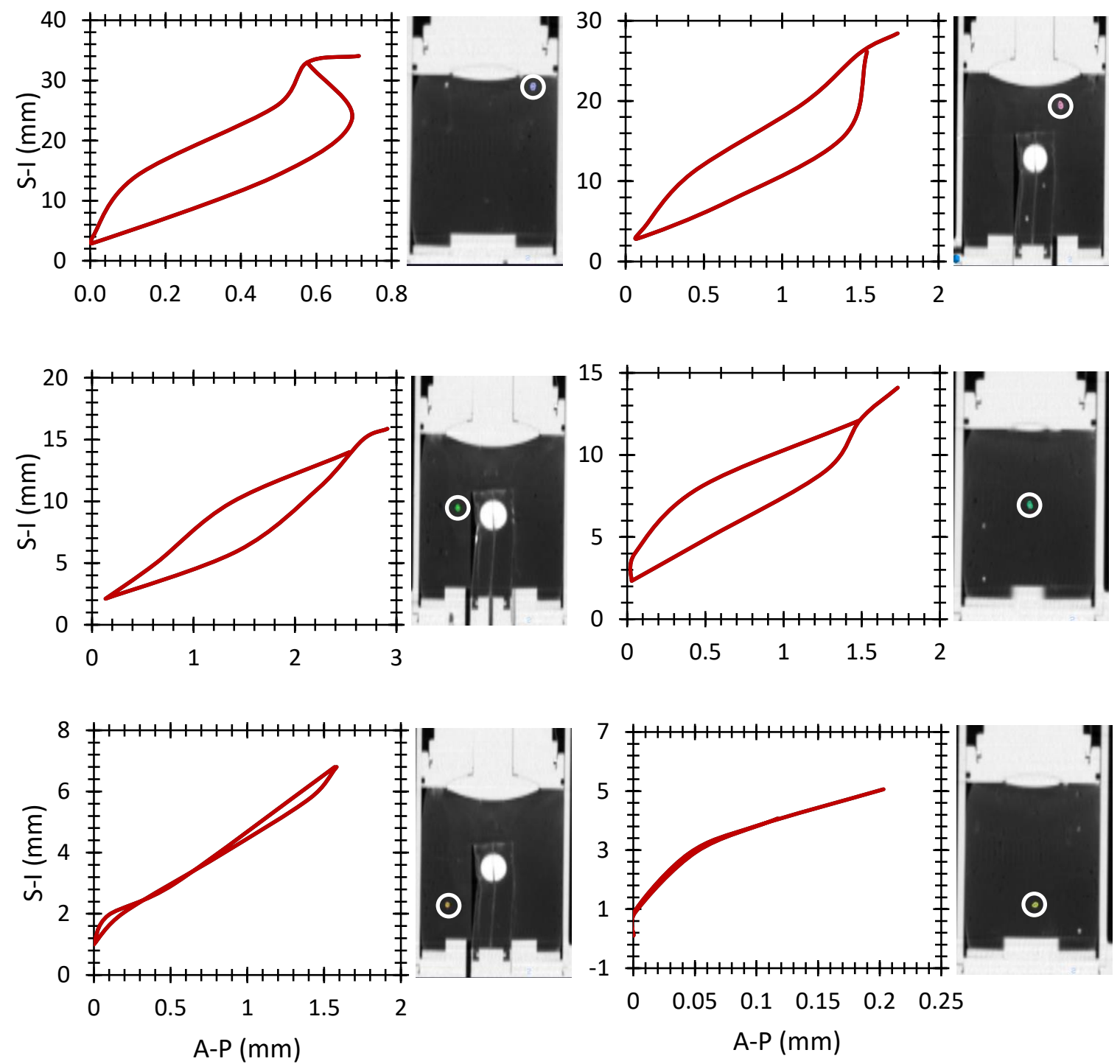

Figure 5.5 Varying levels of hysteresis shown by S-I vs. A-P motion for landmarks (white circles) closest to the piston (top row), middle of the insert (middle row) and furthest from the piston (bottom row). As expected, landmarks closest to the piston show the largest amounts of hysteresis. The data were acquired while the phantom moved with a sinusoidal respiratory motion (period of $3 \mathrm{~s} ; 4 \mathrm{~cm}$ P-P amplitude at the piston).

\subsubsection{Motion assessment and reproducibility}

Measured motion magnitudes in the A-P, L-R and S-I directions along with their reproducibility for the center of the tumor as well as top and bottom surfaces of the plug are shown in Table 5.1. Moreover, 95\% confidence intervals for the range of motion in 3D are shown in Table 5.2 
Table 5.1 Range of motion in 3D for the lung phantom measured by RADPOS detectors at the center of the tumor as well as top and bottom plug surfaces. Values represent the average P-P amplitude of 10 independent measurements along with their standard deviation. Motion in S-I direction as measured from 4DCT images of the EOE and EOI is also presented.

\begin{tabular}{lcccc}
\hline \hline Measurement point & A-P $(\mathrm{cm})$ & L-R $(\mathrm{cm})$ & S-I $(\mathrm{cm})$ & S-I from 4DCT $(\mathrm{cm})$ \\
\hline & & & & \\
Tumor Center & $0.06 \pm 0.01$ & $0.04 \pm 0.01$ & $1.37 \pm 0.06$ & 1.46 \\
Top surface & $0.10 \pm 0.01$ & $0.04 \pm 0.02$ & $1.23 \pm 0.09$ & 1.17 \\
Bottom surface & $0.07 \pm 0.00$ & $0.03 \pm 0.01$ & $1.29 \pm 0.07$ & 1.25 \\
\hline \hline
\end{tabular}

Table 5.2 The $95 \%$ confidence interval for the range of motion presented in Table 5.1.

\begin{tabular}{lccc}
\hline \hline Measurement point & A-P $(\mathrm{cm})$ & L-R $(\mathrm{cm})$ & S-I $(\mathrm{cm})$ \\
\hline & & & \\
Tumor Center & $(0.05,0.07)$ & $(0.03,0.05)$ & $(1.33,1.41)$ \\
Top surface & $(0.09,0.11)$ & $(0.03,0.05)$ & $(1.23,1.29)$ \\
Bottom surface & $(0.07,0.07)$ & $(0.02,0.04)$ & $(1.24,1.34)$ \\
\hline \hline
\end{tabular}

The maximum range of motion, as expected, is along the S-I direction. The S-I displacements measured using 4DCT scans shown in the last column of the table are accurate within the resolution of CT image slices $(2 \mathrm{~mm})$ and agree with the range measured with RADPOS detectors. The A-P and L-R motions are less than $1 \mathrm{~mm}$ because the points of measurements were approximately at the center of the phantom. 


\subsubsection{Motion assessment and reproducibility of the deformable programmable lung}

phantom

Measured motion magnitudes and their reproducibility in S-I, A-P and L-R directions for sinusoidal motion profiles are shown in Figures 5.6-5.8, respectively. Also, calculated values for the 95\% confidence interval for each P-P amplitude are shown in Table 5.3.

Table 5.3 The 95\% confidence intervals of the measured sinusoidal motion for different P-P amplitudes in A-P, L-R and S-I.

\begin{tabular}{cllll}
\hline \hline P-P amplitude $(\mathrm{cm})$ & Measurement point & A-P $(\mathrm{cm})$ & L-R $(\mathrm{cm})$ & S-I $(\mathrm{cm})$ \\
\hline \multirow{4}{*}{$1 \mathrm{~cm}$} & & & \\
& Tumor Center & $(0.20,0.21)$ & $(0.10,0.11)$ & $(4.30,4.37)$ \\
& Top surface & $(0.21,0.25)$ & $(0.12,0.15)$ & $(4.10,4.14)$ \\
& Bottom surface & $(0.21,0.25)$ & $(0.10,0.13)$ & $(4.26,4.32)$ \\
\hline \multirow{3}{*}{$1.5 \mathrm{~cm}$} & Tumor Center & $(0.21,0.25)$ & $(0.11,0.14)$ & $(6.79,6.86)$ \\
& Top surface & $(0.26,0.29)$ & $(0.15,0.19)$ & $(6.60,6.65)$ \\
& Bottom surface & $(0.23,0.27)$ & $(0.15,0.18)$ & $(6.78,6.85)$ \\
\hline \multirow{3}{*}{$2 \mathrm{~cm}$} & Tumor Center & $(0.43,0.47)$ & $(0.12,0.17)$ & $(9.03,9.16)$ \\
& Top surface & $(0.45,0.50)$ & $(0.18,0.21)$ & $(8.95,9.05)$ \\
& Bottom surface & $(0.43,0.48)$ & $(0.16,0.20)$ & $(8.98,9.09)$ \\
\hline \multirow{3}{*}{$2.5 \mathrm{~cm}$} & Tumor Center & $(0.65,0.69)$ & $(0.16,0.22)$ & $(11.36,11.45)$ \\
& Top surface & $(0.66,0.71)$ & $(0.19,0.24)$ & $(11.18,11.29)$ \\
& Bottom surface & $(0.63,0.67)$ & $(0.18,0.23)$ & $(11.22,11.33)$ \\
\hline \multirow{2}{*}{$3 \mathrm{~cm}$} & Tumor Center & $(0.79,0.82)$ & $(0.19,0.23)$ & $(13.16,13.27)$ \\
& Top surface & $(0.78,0.83)$ & $(0.21,0.25)$ & $(13.01,13.12)$ \\
& Bottom surface & $(0.80,0.83)$ & $(0.21,0.25)$ & $(13.04,13.12)$ \\
\hline \hline
\end{tabular}



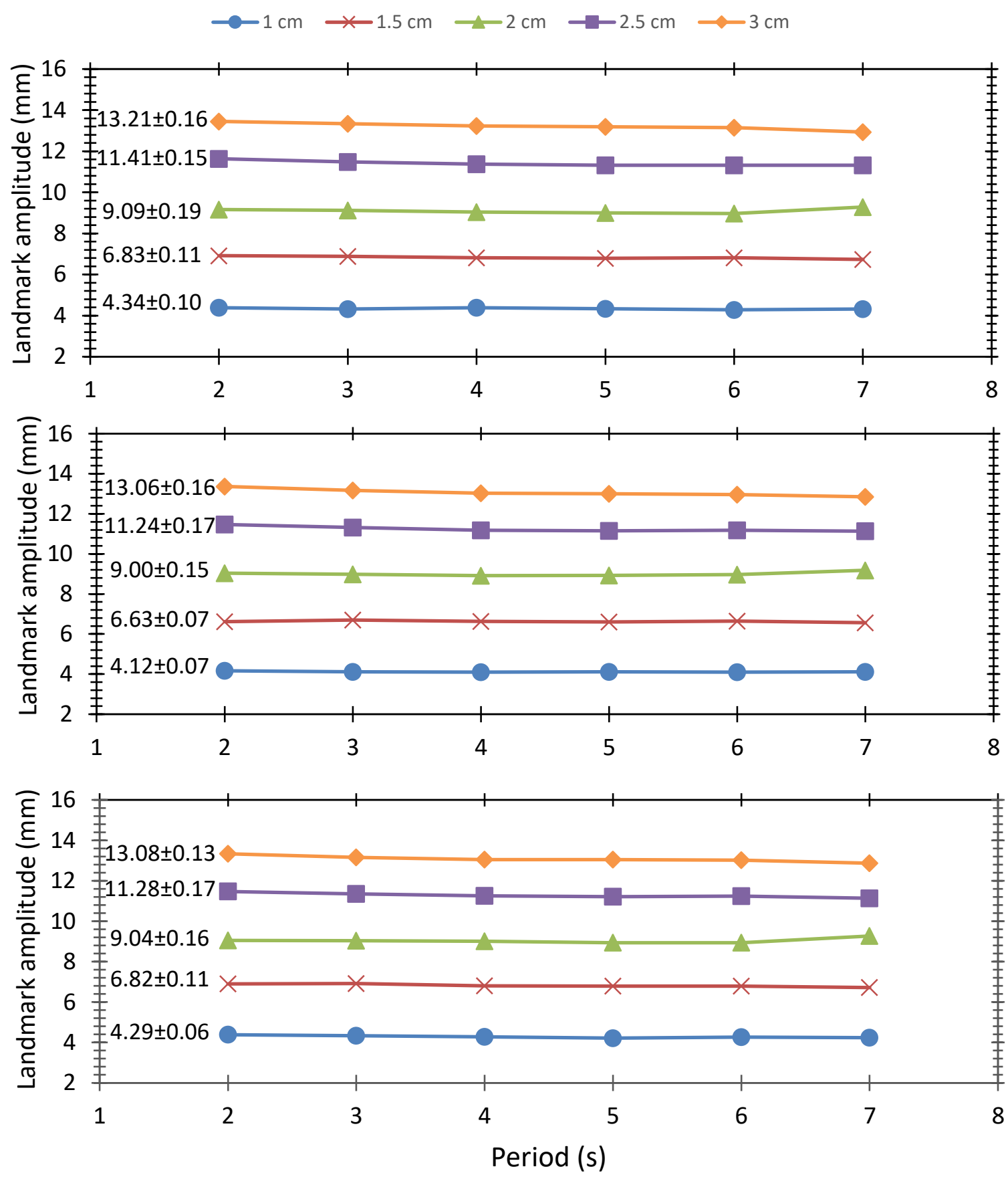

Figure 5.6 Range of S-I motion measured by RADPOS detectors at the tumor (top), top (middle) and bottom (bottom) plug surfaces vs period of sinusoidal motion. Average P-P amplitude values and their reproducibility (combined standard deviations for one amplitude is the square root of the sum of the square of individual standard deviations) are shown on the plots. 

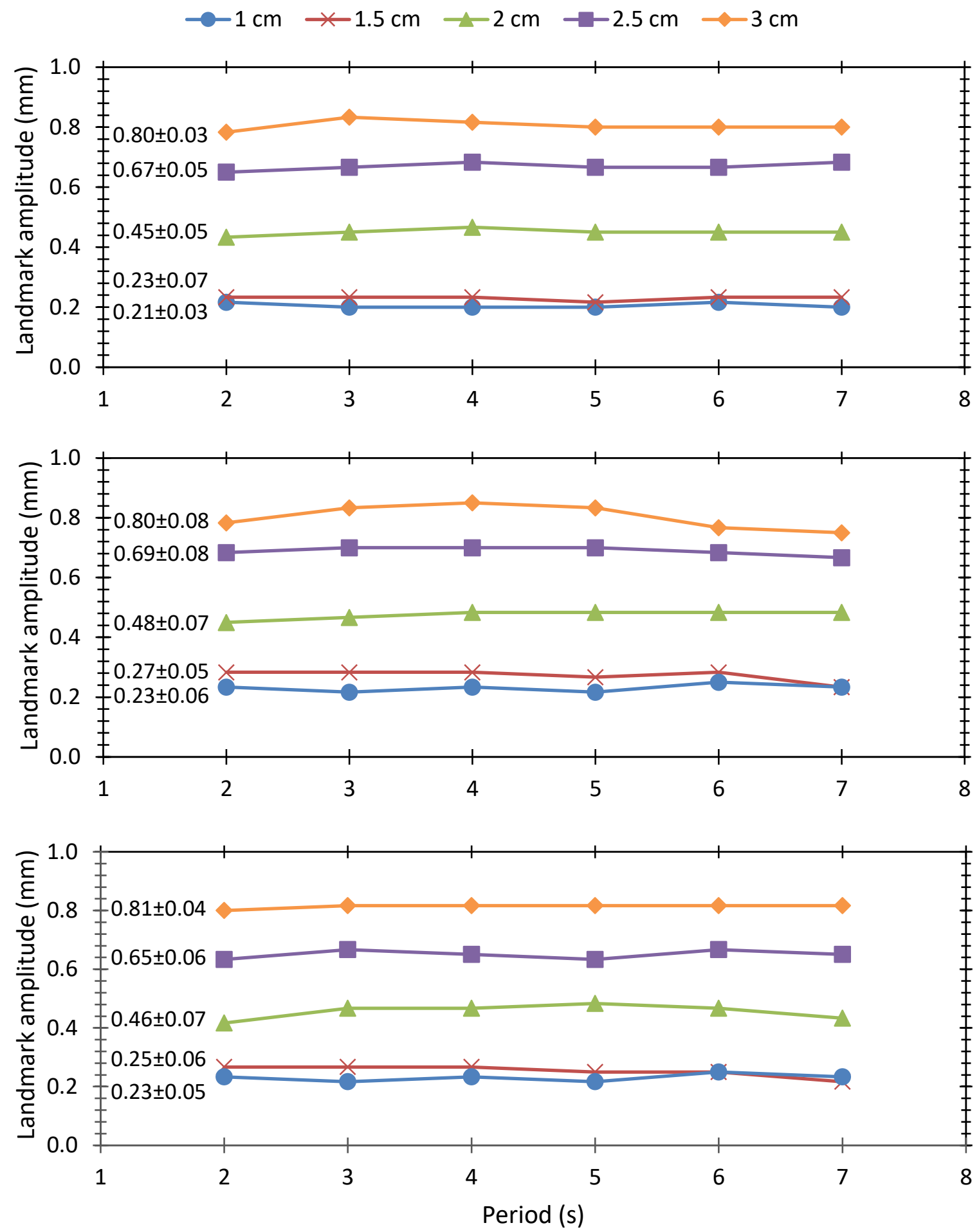

Figure 5.7 Range of A-P motion measured by RADPOS detectors at the tumor (top), top (middle) and bottom (bottom) plug surfaces vs period of sinusoidal motion. Average P-P amplitude values and their reproducibility (combined standard deviations for one amplitude is the square root of the sum of the square of individual standard deviations) are shown on the plots. 

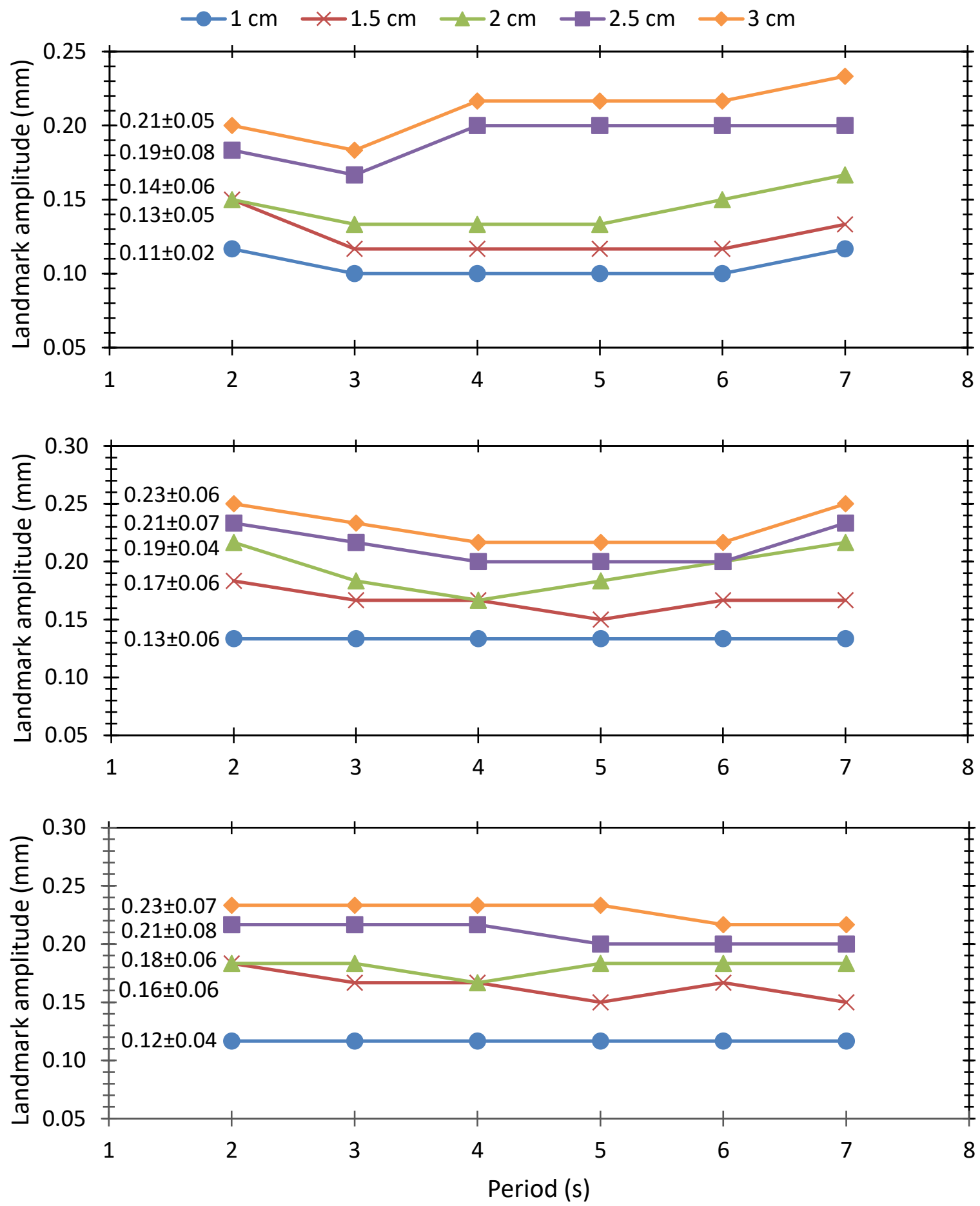

Figure 5.8 Range of L-R motion measured by RADPOS detectors at the tumor (top), top (middle) and bottom (bottom) plug surfaces vs period of sinusoidal motion. Average P-P amplitude values and their reproducibility (combined standard deviations for one amplitude is the square root of the sum of the square of individual standard deviations) are shown on the plots. 
Overall, the reproducibility of the P-P amplitudes was measured to be within 0.2 , 0.1 and $0.1 \mathrm{~mm}$ for motions in S-I, A-P and L-R directions, respectively. The S-I motion measured by all three RADPOS detectors is very similar while we can observe differences in the motion measured in A-P and L-R directions. This is evidence of different deformations at different points of the phantom. Maximum intra-day and inter-day variation for S-I, A-P and L-R directions for all three RADPOS detectors are shown in Table 5.4.

Table 5.4 Inter- and intra-day amplitude variations in S-I, A-P and L-R directions for tumor, top and bottom RADPOS detectors for sinusoidal motion profiles.

\begin{tabular}{lllll}
\hline \hline & Measurement point & A-P $(\mathrm{cm})$ & L-R (cm) & S-I (cm) \\
\hline \multirow{4}{*}{ Inter-day } & Tumor Center & 0.02 & 0.02 & 0.09 \\
& Top surface & 0.03 & 0.02 & 0.08 \\
& Bottom surface & 0.02 & 0.02 & 0.07 \\
\hline \multirow{6}{*}{ Intra-day } & & & & \\
& Tumor Center & 0.01 & 0.01 & 0.02 \\
& Top surface & 0.02 & 0.01 & 0.03 \\
& Bottom surface & 0.02 & 0.02 & 0.02 \\
\hline \hline
\end{tabular}

Figure 5.9 compares the motion trace of the tumor in the S-I direction measured by RADPOS with the recorded diaphragm motion for three of the simulated irregular profiles at a P-P diaphragm amplitude of $3 \mathrm{~cm}$. For visibility purposes, only $80 \mathrm{~s}$ of the traces are shown here. The motion traces measured at the top and bottom surfaces of the plug are equally similar to the trace measured at the tumor center. 


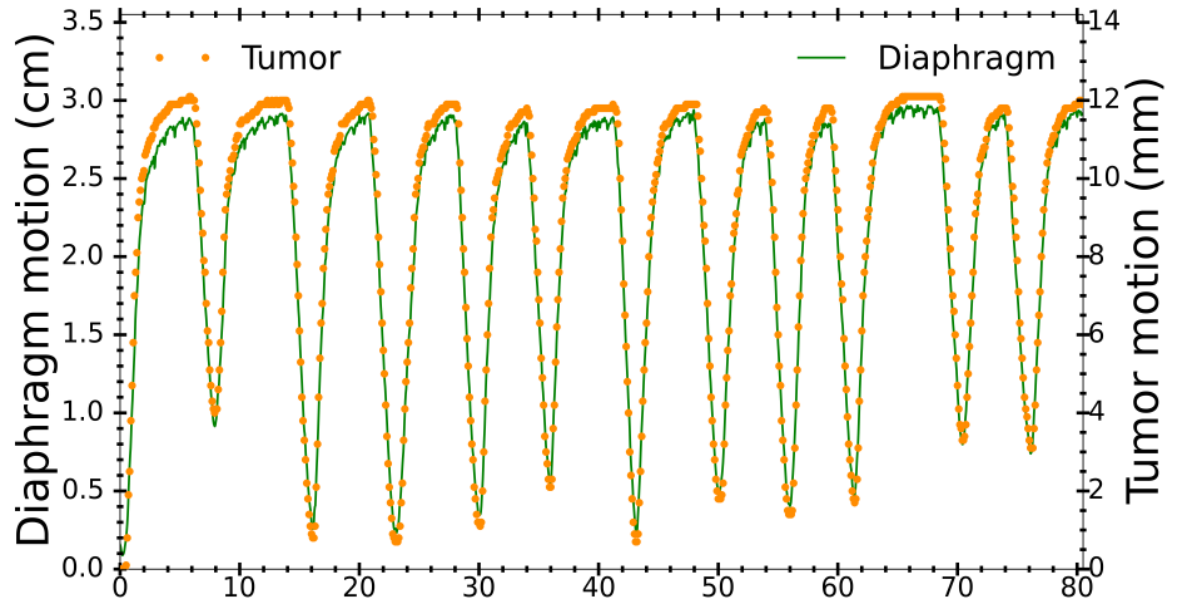

(a)

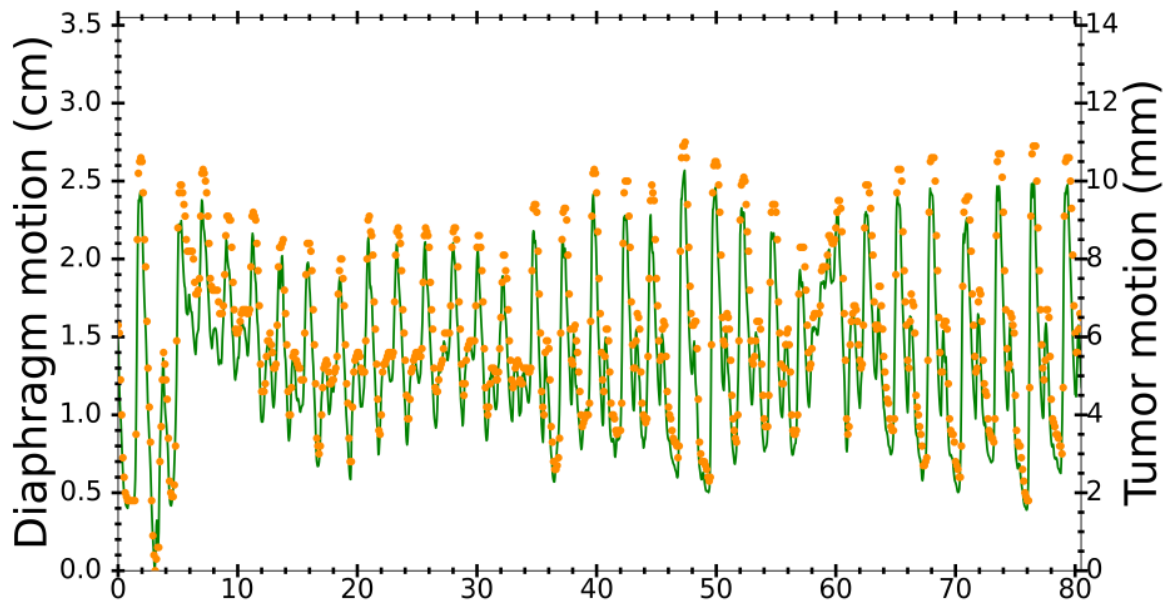

(b)

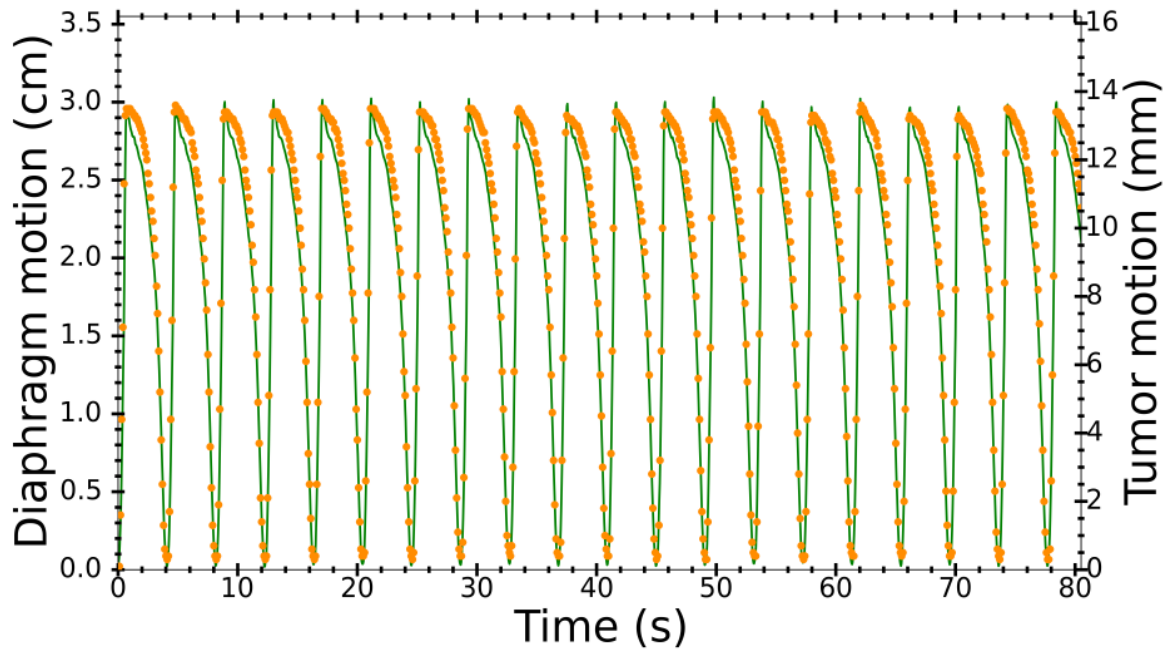

(c)

Figure 5.9 Comparison of the tumor (orange dots) and diaphragm (green line) motion traces for respiratory motion profiles that are (a) typical, (b) highly irregular and (c) show large hysteresis between the tumor and diaphragm motion at a P-P diaphragm amplitude of $3 \mathrm{~cm}$. 
No lag was observed in the foam response due to the piston motion.

Irregular motion profiles present amplitude variations over the full course of the profile. For the three respiratory motion profiles shown in Figure 5.9, these amplitudes are calculated to be $9.78 \pm 1.19,6.56 \pm 1.90$ and $13.18 \pm 0.18 \mathrm{~mm}$, respectively.

Reproducibility of the average P-P amplitudes as well as the amplitude variations in 3D from all simulated profiles are shown in Table 5.5 for 3 RADPOS detectors.

Table 5.5 Reproducibility of the average P-P amplitude and amplitude variations in S-I, A-P and L-R directions for tumor, top and bottom RADPOS detectors for irregular motion profiles. Values represent the standard deviation of the average $P$-P amplitude and average value of amplitude variations across individual motion traces, respectively.

\begin{tabular}{cllll}
\hline \hline & Measurement point & A-P $(\mathrm{cm})$ & L-R $(\mathrm{cm})$ & S-I (cm) \\
\hline & Tumor Center & 0.025 & 0.012 & 0.038 \\
$\begin{array}{c}\text { Average P-P } \\
\text { amplitude }\end{array}$ & Top surface & 0.023 & 0.012 & 0.039 \\
& Bottom surface & 0.022 & 0.010 & 0.037 \\
& & & & 0.015 \\
P-P amplitude & Tumor Center & 0.004 & 0.004 & 0.012 \\
variations & Top surface & 0.005 & 0.004 & 0.014 \\
\hline \hline
\end{tabular}


Maximum inter-day variations of the amplitude are shown in Table 5.6 for S-I, A$\mathrm{P}$ and L-R directions for all three RADPOS detectors.

Table 5.6 Inter-day amplitude variations in S-I, A-P and L-R directions for tumor, top and bottom RADPOS detectors for irregular motion profiles.

\begin{tabular}{clccc}
\hline \hline & Measurement point & A-P $(\mathrm{cm})$ & L-R $(\mathrm{cm})$ & S-I $(\mathrm{cm})$ \\
\hline & Tumor Center & 0.027 & 0.028 & 0.087 \\
$\begin{array}{c}\text { Average P-P } \\
\text { amplitude }\end{array}$ & Top surface & 0.031 & 0.028 & 0.094 \\
& Bottom surface & 0.024 & 0.025 & 0.093 \\
& & & & \\
& Tumor Center & 0.010 & 0.013 & 0.030 \\
P-P amplitude & Top surface & 0.013 & 0.014 & 0.030 \\
& Bottom surface & 0.008 & 0.014 & 0.036 \\
\hline \hline
\end{tabular}

One of the applications of RADPOS, as a real-time motion detector, is to enable us to correlate the motion of the diaphragm to the motion of any point of interest (e.g. tumor) inside the phantom, during one or several respiratory phases. Figure 5.10 shows such correlations for sinusoidal (periods of 2 and $7 \mathrm{~s}$ ) and irregular motion profiles shown in Figure 5.9 (a and c) in the form of hysteresis plots for S-I direction. 


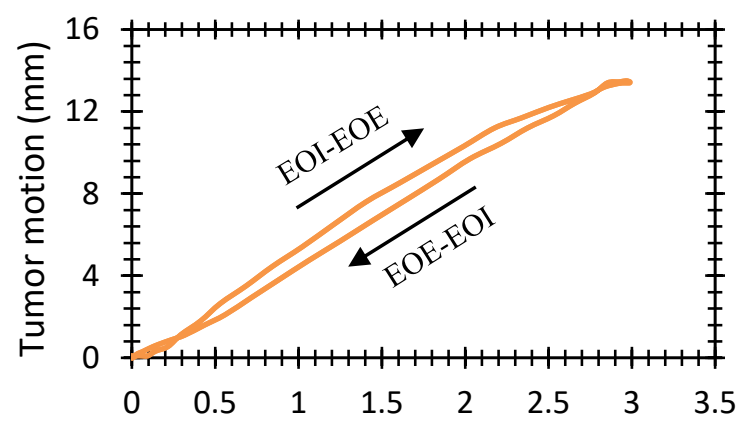

(a)

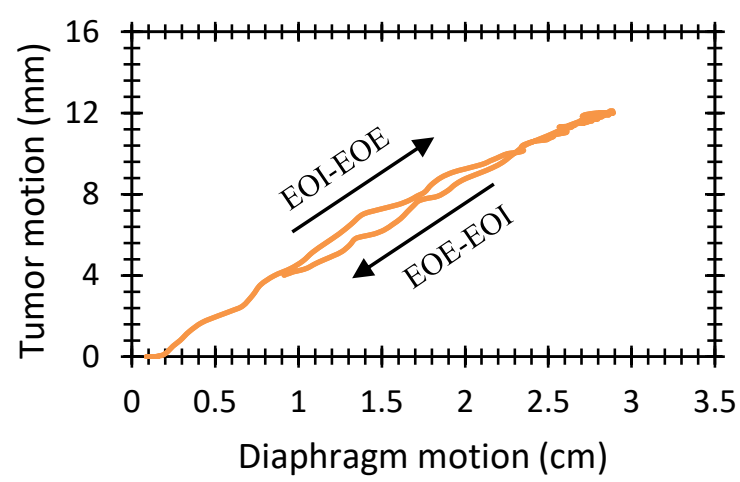

(c)

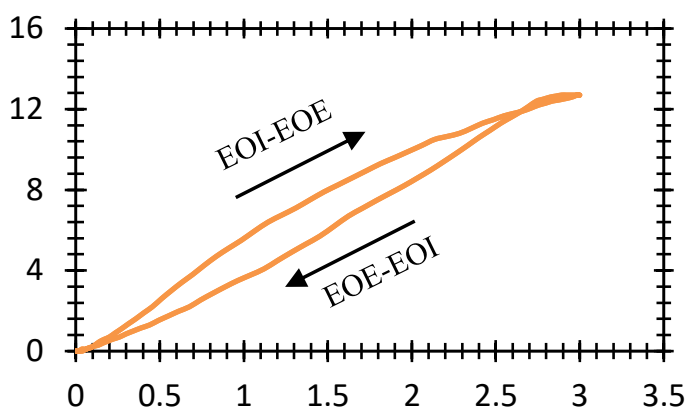

(b)

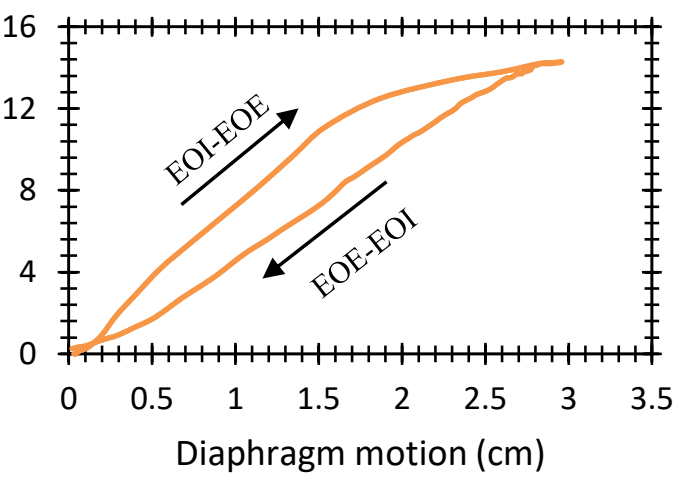

(d)

Figure 5.10 Correlation curves between motion of the tumor and diaphragm for P-P diaphragm amplitude of $3 \mathrm{~cm}$. Correlations are shown in the form of hysteresis plots for sinusoidal motion profiles with periods of (a) $4 \mathrm{~s}$, (b) $7 \mathrm{~s}$ as well as (c) typical (Figure 5.9 (a)) and (d) large hysteresis between the tumor and diaphragm motion (Figure 5.9 (c)) respiratory motion profiles.

\subsection{Discussion}

Although the Quasar phantom is capable of simulating translations of the anatomy due to respiration in the S-I direction, it lacks the ability to simulate the deformations that occur as well. To simulate a more realistic lung anatomy, a deformable lung phantom composed of compressible tissue-equivalent foam containing a moving non-rigid tumor was constructed and characterized for dosimetry purposes. Design of the phantom allowed inclusion/removal of film and RADPOS for dose and position measurements. The initial version of the phantom used a DC motor to compress/decompress the foam through a piston and was only capable of simulating sinusoidal motion profiles with a P-P amplitude 
of $4 \mathrm{~cm}$. Recent modifications to the phantom (i.e. replacing the DC motor with a programmable servo motor), enabled us to simulate both sinusoidal and irregular respiratory motion profiles at different amplitudes. The phantom is simple to set up and overall showed good motion/setup reproducibility $(\sim 1 \mathrm{~mm})$ in 3D. Moreover, the phantom showed several levels of hysteresis which were extracted through landmarks injected throughout the phantom. In addition, using RADPOS inside the phantom was helpful in detecting the non-linear motion behavior of the phantom by correlating the motion of different measurement points inside the phantom to the motion of diaphragm.

\subsection{Conclusions}

A novel reproducible deformable lung phantom with capability to hold film and RADPOS was developed and validated. The phantom is capable of simulating sinusoidal and realistic respiratory motion profiles at several amplitudes. In combination with RADPOS, the phantom can be a useful tool for dosimetric validations of 4DMC simulations and motion prediction/modeling of different points of the phantom. 


\section{Chapter 6}

\section{Experimental verification of 4D Monte Carlo simulations of dose delivery to moving/deforming phantoms}

\subsection{Introduction}

As previously explained in Chapter 1, 4D dose accumulation methods are the most accurate approach to account for the effect of respiratory motion on the delivered dose as they take all three impacts of respiratory motion, dose blurring, dose deformations and interplay effects, into account. The concept of 4D dose accumulation was first introduced by Brock et al..$^{59}$ but it has been since investigated by many other groups as well ${ }^{25,60,68,152-}$ 154. These groups studied the use of deformation vector fields (DVFs) acquired from deformable image registration (DIR) to map dose distributions calculated on multiple respiratory states to a reference anatomy where they are summed to give a cumulative dose distribution. However, the interplay effect was not included in these studies. Paganetti et $a l .{ }^{155}$ performed 4D Monte Carlo (MC) simulations, using the GEANT4 Monte Carlo code, to model proton therapy delivery. Their simulations included interplay effect by continuously updating both the beam delivery and dose calculation geometries as a function of time spent in each respiratory phase. Rao et al. ${ }^{30}$ focused on IMRT and VMAT deliveries and determined the beam segment corresponding to each respiratory phase. The dose from each segment was calculated on the appropriate respiratory phase.

One challenge of mapping dose between anatomies is that the DIR does not always result in a 1-to-1 voxel correspondence between the source and the reference respiratory phases. During a registration process, voxels might split or merge while determining the 
correlation between images and as a result, the dose calculation grid may not be conserved. Thus, tracking the dose deposited in a certain voxel in the reference respiratory phase to its source will not be straightforward. A commonly used dose mapping approach is the dose interpolation method (DIM) $)^{152}$. A drawback of using dose interpolation in the case where voxels split or merge is that inaccuracies will occur in regions of dose or density gradient ${ }^{156}$. More accurate approaches are the energy mass congruent mapping (EMCM) $)^{156,157}$ and voxel warping method (VWM $)^{84}$. Both approaches make use of DVFs to calculate the accumulated dose. In EMCM, DVFs are used to map the mass and energy deposited in voxels on each respiratory phase to a reference phase. VWM uses DVFs to deform voxels from the reference geometry to reproduce each respiratory phase. Both methods assure conservation of the energy deposited as well as the mass on each respiratory phase, and as a result, a more accurate cumulative dose calculation on the reference phase.

Regardless of the methodology used to calculate the cumulative dose on a moving/deforming anatomy, quality assurance of the DIR is an important step, since accuracy of DIR impacts the accuracy of dose calculations. Visual evaluation of the DIR is a preliminary and common test ${ }^{158}$. There are also quantitative methods including landmark tracking ${ }^{59,147,158-161}$, contour comparison ${ }^{158,160}$ as well as benchmarking against known deformations generated by a computational or physical phantom ${ }^{158,159,162}$.

An essential step in verifying 4D dose calculation algorithms is experimental verification through appropriate measurements. Belec et al. ${ }^{163}$ compared measurements of a sweeping field delivered to a Quasar respiratory motion phantom moving with a sinusoidal motion with $2 \mathrm{~cm}$ amplitude and $8 \mathrm{~s}$ period against $\mathrm{MC}$ calculations based on a method similar to EMCM. They modeled the continuous motion of the beam using a 
position-probability sampling (PPS) technique ${ }^{164}$. A randomly sampled time variable between 0 and 1 was associated with each particle which was used to sample the linac geometry settings specified as a function of normalized cumulative MU. The time variable was then used to interpolate the DVF and voxel densities to more closely model changes associated with continuous motion. In order to minimize the error caused by linear interpolation of the voxel densities, the motion between respiratory phases was reduced to be smaller than voxel sizes. To decrease the motion between breathing phases, they used the DVFs between each breathing phase and the reference phase as well as its neighboring phases to map the 10 original phases of the 4DCT scans to 11 new breathing phases with equally spaced position of the tumor. Zhong et al. ${ }^{161}$ recently published their results on experimental verifications of a MC dose accumulation algorithm using EMCM. They designed a phantom made of sponge insert and tissue-equivalent tumor composed of bolus material and irradiated it with a static beam in stationary and deformed modes. Dose was measured with thermoluminescent dosimeters (TLD) placed at multiple locations in the phantom.

Vinogradskiy et al. ${ }^{151,165}$ utilized a deformable phantom, which was previously described in Chapter 5 , to verify a 4D dose calculation algorithm implemented in a research version of Pinnacle ${ }^{3}$ treatment planning system (Philips Radiation Oncology System, Fitchburg, WI, USA). They compared measured and calculated dose distributions for sinusoidal and irregular motion patterns during conformal and IMRT beam deliveries. In a study conducted by Niu et al. ${ }^{166}$ a gel dosimeter was irradiated with a conformal plan while being compressed at different amplitudes and the resultant dose distributions were 
compared against 3D dose accumulation calculations using a previously developed dose accumulation algorithm ${ }^{59}$.

This chapter presents experimental validation of a 4D Monte Carlo dose calculation tool, using the 4DdefDOSXYZnrc code, to reconstruct the dose delivered to continuously rigidly moving and deforming lung phantoms during sinusoidal and realistic respiratory motion profiles. Before a treatment verification tool can be used in the clinic, it is important to validate its accuracy in calculating the cumulative delivered dose. For these validations, the phantoms introduced in Chapter 5 were used to measure the dose delivered during static and VMAT plan deliveries. A preliminary validation was performed using the rigidly moving Quasar phantom with sinusoidal breathing motion. The work is then extended to more realistic situations with the programmable deformable phantom. The measured dose was then compared against corresponding 4D Monte Carlo simulations to evaluate the accuracy of the code.

\subsection{Materials and Methods}

\subsubsection{Film and RADPOS calibration}

The EBT3 film and RADPOS detectors used in this work were cross-calibrated against an A1SL ionization chamber using a $6 \mathrm{MV}$ photon beam and a field size of $10 \times 10 \mathrm{~cm}^{2}$. Film and RADPOS were placed at a depth of $5 \mathrm{~cm}$, and the A1SL chamber was placed at a depth of $10 \mathrm{~cm}$ in a Solid Water phantom at an SSD of $100 \mathrm{~cm}$ as shown in Figure 6.1. After three irradiations of $100 \mathrm{MU}$ for RADPOS detectors and eight irradiations of $0,5,25,50,75,100,110$, and $130 \mathrm{MU}$ for film, dose to water $\left(\mathrm{D}_{\mathrm{w}}\right)$ at the position of RADPOS and film was determined using the PDD curves available in the clinic. 


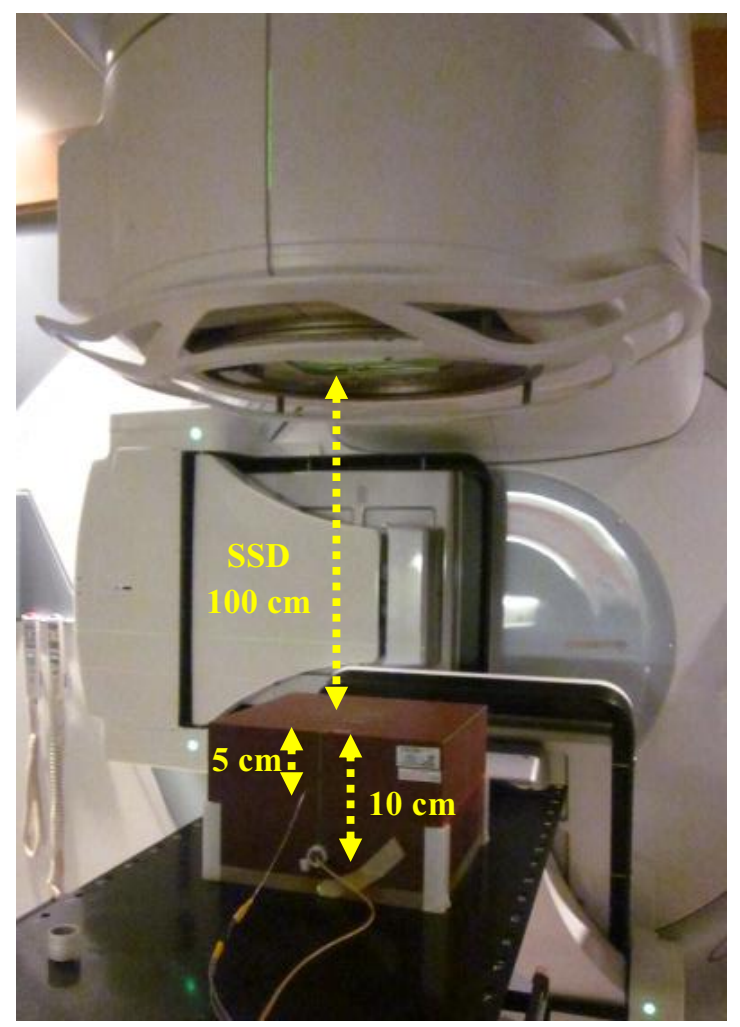

Figure 6.1 Measurement setup used for film and RADPOS calibration. Film and RADPOS were placed at $5 \mathrm{~cm}$ depth of the Solid Water phantom while the ion chamber was at $10 \mathrm{~cm}$ at an SSD setup of $100 \mathrm{~cm}$.

The film analysis and calibration were performed according to the procedures described in Section 2.3 and the calibration curve, similar to the one in Figure 2.4, was derived. The calibration coefficient $(\mathrm{cGy} / \mathrm{mV})$ for RADPOS was derived through the formalism described in Equation 2.5.

Summaries of the various sources of uncertainty that contribute to the total dosimetric uncertainty of film and RADPOS measurements are presented in Tables 6.1 and 6.2 , respectively. The uncertainty value for $\mathrm{N}_{\mathrm{D}, \mathrm{w}}$ was derived from corresponding data in the clinic. 
Table 6.1 Summary of various sources of dosimetric uncertainty for film measurements.

\begin{tabular}{|c|c|c|}
\hline & Source & Value \\
\hline \multirow{5}{*}{ Beam delivery ${ }^{167}$} & SSD setting & $0.10 \%$ \\
\hline & Depth setting & $0.17 \%$ \\
\hline & Field size setting & $0.10 \%$ \\
\hline & $\mathrm{P}_{\mathrm{T}, \mathrm{P}}$ & $0.10 \%$ \\
\hline & Humidity & $0.05 \%$ \\
\hline \multirow{5}{*}{$\begin{array}{l}\text { Beam dosimetry } \\
\text { calibration }\end{array}$} & $\mathrm{N}_{\mathrm{D}, \mathrm{w}}$ & $1.00 \%$ \\
\hline & $\mathrm{K}_{\mathrm{Q}}^{167}$ & $0.40 \%$ \\
\hline & Assignment of $\mathrm{K}_{\mathrm{Q}}{ }^{167}$ & $0.10 \%$ \\
\hline & Leakage current ${ }^{167}$ & $0.05 \%$ \\
\hline & Solid Water phantom material variability ${ }^{168}$ & $0.70 \%$ \\
\hline \multirow[t]{4}{*}{ User dependent ${ }^{167}$} & & $0.30 \%$ \\
\hline & Scanner uniformity & $0.30 \%$ \\
\hline & Lateral correction & $1.00 \%$ \\
\hline & Fit accuracy (netOD to dose) & $0.50 \%$ \\
\hline \multirow{5}{*}{ EBT3 film ${ }^{72}$} & Intra-batch variations & $0.20 \%$ \\
\hline & Background & $0.50 \%$ \\
\hline & Energy dependence & $0.50 \%$ \\
\hline & Angular dependence & $0.50 \%$ \\
\hline & Intrinsic film inhomogeneity & $1.10 \%$ \\
\hline \multicolumn{2}{|l|}{ Total Film } & $2.30 \%$ \\
\hline
\end{tabular}


Table 6.2 Summary of various sources of dosimetric uncertainty for RADPOS measurements.

\begin{tabular}{|c|c|c|}
\hline & Source & Value \\
\hline \multirow{5}{*}{ Beam delivery ${ }^{167}$} & SSD setting & $0.10 \%$ \\
\hline & Depth setting & $0.17 \%$ \\
\hline & Field size setting & $0.10 \%$ \\
\hline & $\mathrm{P}_{\mathrm{T}, \mathrm{P}}$ & $0.10 \%$ \\
\hline & Humidity & $0.05 \%$ \\
\hline \multirow{5}{*}{$\begin{array}{l}\text { Beam dosimetry } \\
\text { calibration }\end{array}$} & $\mathrm{N}_{\mathrm{D}, \mathrm{w}}$ & $1.00 \%$ \\
\hline & $\mathrm{K}_{\mathrm{Q}}{ }^{167}$ & $0.40 \%$ \\
\hline & Assignment of $\mathrm{K}_{Q}{ }^{167}$ & $0.10 \%$ \\
\hline & Leakage current ${ }^{167}$ & $0.05 \%$ \\
\hline & Solid Water phantom material variability 168 & $0.70 \%$ \\
\hline User dependent ${ }^{167}$ & & $0.30 \%$ \\
\hline \multirow{2}{*}{ RADPOS \#1 } & IC reading & $0.16 \%$ \\
\hline & RADPOS reproducibility & $0.48 \%$ \\
\hline Total RADPOS \#1 & & $1.40 \%$ \\
\hline \multirow{2}{*}{ RADPOS \#2 } & IC reading & $0.06 \%$ \\
\hline & RADPOS reproducibility & $1.79 \%$ \\
\hline Total RADPOS \#2 & & $2.24 \%$ \\
\hline \multirow{2}{*}{ RADPOS \#3 } & IC reading & $0.06 \%$ \\
\hline & RADPOS reproducibility & $1.92 \%$ \\
\hline Total RADPOS \#3 & & $2.35 \%$ \\
\hline \multirow{2}{*}{ RADPOS \#4 } & IC reading & $0.06 \%$ \\
\hline & RADPOS reproducibility & $1.95 \%$ \\
\hline Total RADPOS \#4 & & $2.37 \%$ \\
\hline
\end{tabular}




\subsubsection{Quasar phantom}

\subsubsection{3DCT acquisition}

3DCT scans of the Quasar phantom were acquired at a standard image resolution of $0.0625 \times 0.0625 \times 0.1 \mathrm{~cm}^{3}$ resulting in an image matrix of $512 \times 512 \times 246$ voxels. The center of the tumor inside the lung insert was positioned at the zero-amplitude position. Pitch values and the gantry rotation times for the scans were 0.95 and $0.75 \mathrm{~s}$, respectively.

\subsubsection{Displacement vector generation}

The common practice to generate DVF between two respiratory phases is to use deformable image registration. However, as the Quasar lung insert moves rigidly, a DIR algorithm could introduce errors in the DVFs. To avoid such errors, displacement vectors that exactly modeled this rigid translation (P-P amplitude and direction) were generated with an in-house Python script.

\subsubsection{Treatment plans}

Two treatment plans, static and VMAT, for the $6 \mathrm{MV}$ photon beam of an Elekta Infinity linac with Agility MLC were created on the static 3DCT of the Quasar phantom to deliver 100 cGy to the center of the tumor. A gross target volume (GTV) was created by delineating the tumor. No margins were added to compensate for motion. Both treatment plans were designed to cover the GTV.

The first treatment plan was a static $4 \times 4 \mathrm{~cm}^{2}$ square field that was created using $\mathrm{XiO}$ V.4.7 (Elekta AB, Stockholm, Sweden) where dose was calculated using a fast point-kernel Convolution/Superposition $(\mathrm{C} / \mathrm{S})$ algorithm ${ }^{169}$. In this algorithm the dose distribution is calculated by convolving a spatially invariant point-dose kernel with the total energy 
released by incident photons at each point of the phantom. The point-dose kernel is a Monte Carlo-generated dose distribution, resulting from a photon interaction at a point in the middle of a large sphere of water and is stored in the treatment planning system. To improve the dose calculation speed, a multigrid dose calculation approach is implemented. In this approach, dose is only calculated at points where it is necessary (i.e. every other point inside/outside the beam and every point inside regions with high dose/tissue density gradient) and interpolation is used for reasonable estimate of dose at other points.

The second treatment plan was a VMAT plan with 45 control points that was created in Monaco V.5.10.02 (Elekta AB., Stockholm, Sweden). The start and stop angles of the VMAT delivery arc were $180^{\circ}$ with an angular spacing of $8^{\circ}$ between control points. Dose in Monaco was calculated using XVMC (X-ray voxel Monte Carlo) algorithm. In order to be consistent with measurements (film and RADPOS calibrated in Solid Water), dose to water $\left(D_{w}\right)$ was calculated in Monaco using a $2 \mathrm{~mm}$ dose calculation grid to achieve a statistical uncertainty of $1.0 \%$.

Static and VMAT plans covered the GTV with $85 \%$ and $80 \%$ isodose lines, respectively. The dose distributions for the static $4 \times 4 \mathrm{~cm}^{2}$ square field from $\mathrm{XiO}$ and the VMAT plan from Monaco are shown in Figures 6.2 and 6.3. 


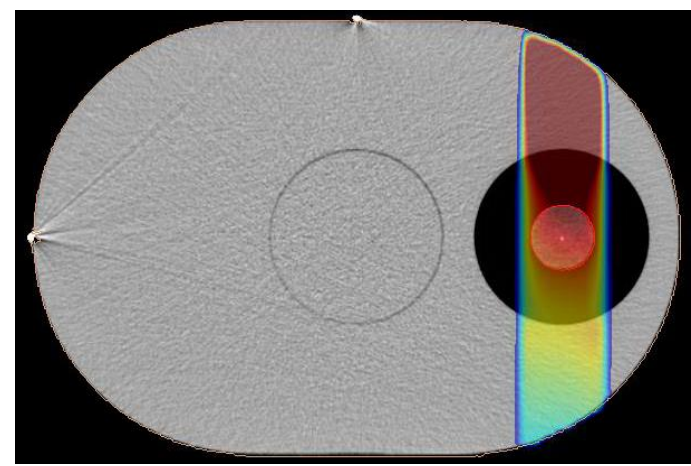

(a)

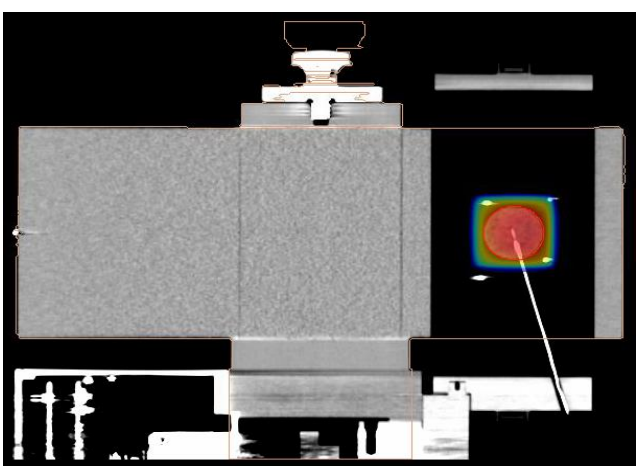

(b)
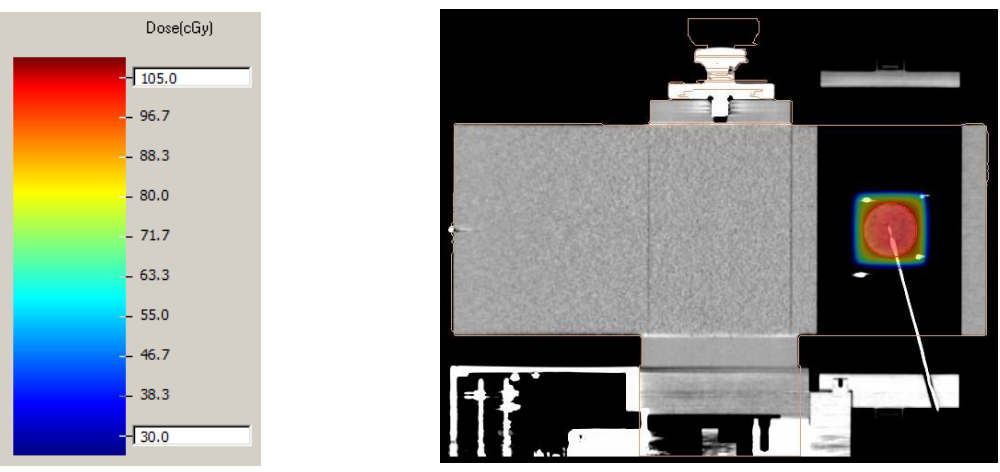

(c)

Figure 6.2 Static $4 \times 4 \mathrm{~cm}^{2}$ square plan: dose distributions from $\mathrm{XiO}$ on (a) axial, (b) coronal, and (c) sagittal planes. 


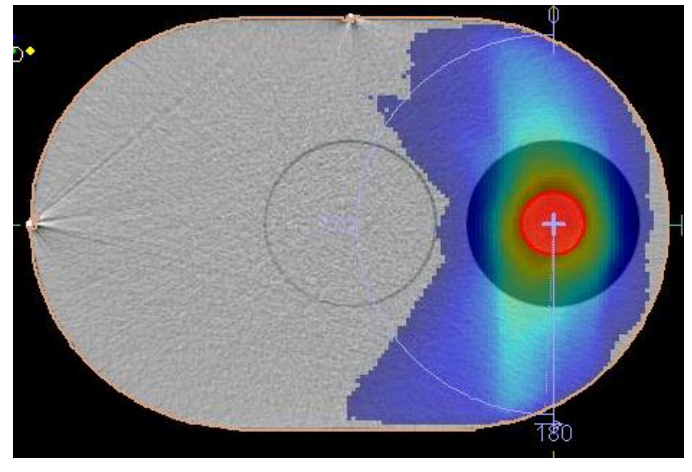

(a)

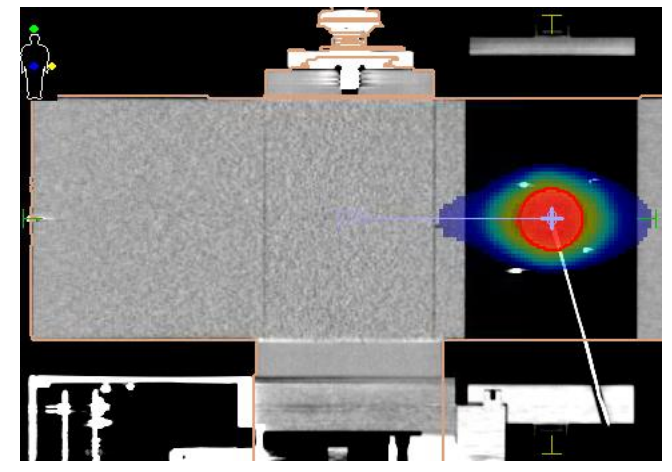

(b)
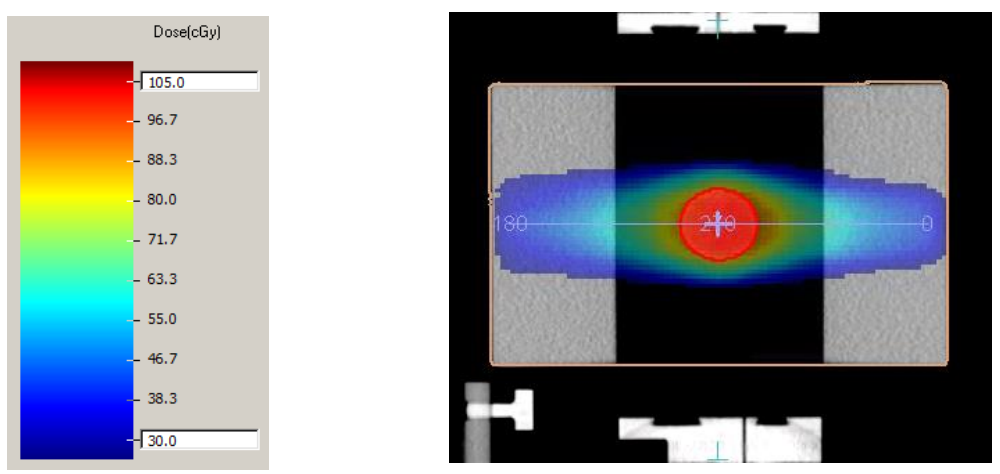

(c)

Figure 6.3 VMAT plan: dose distributions from Monaco on (a) axial, (b) coronal, and (c) sagittal planes. 


\subsubsection{Phantom irradiations}

Both treatment plans were exported to the Elekta MOSAIQ RadOnc system and delivered to the Quasar phantom on an Elekta Infinity linac. The $4 \times 4 \mathrm{~cm}^{2}$ square plan delivered 123.2 MU at a nominal dose rate of $600 \mathrm{MU} / \mathrm{min}$. The VMAT plan delivered 172.3 MU with a varying dose rate. Delivery log files (IAN V.2; Elekta AB., Stockholm, Sweden) containing cumulative delivered MU, gantry, collimator, MLC, jaws, and table positions at $40 \mathrm{~ms}$ intervals were recorded during each delivery. For both treatment plans, irradiations were performed with the Quasar in stationary (no motion) and breathing (sinusoidal motion: $1.8 \mathrm{~cm}$ respiratory amplitude, $3.3 \mathrm{~s}$ period) states. To align the phantom on the couch, the center of the sphere inside the lung insert was positioned at the zeroamplitude position and was aligned with the beam isocenter (Figure 6.4).
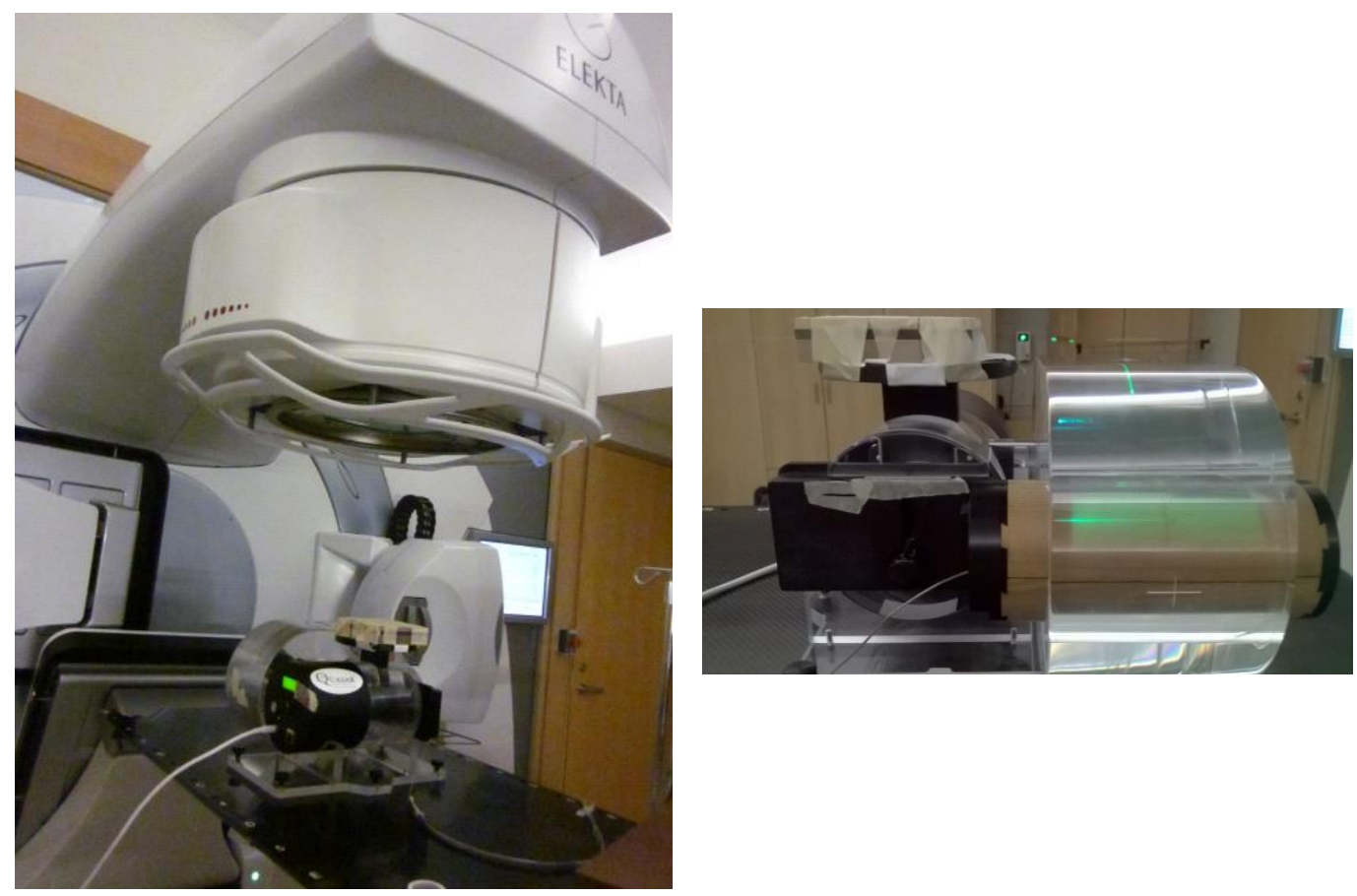

Figure 6.4 Setup for Quasar phantom irradiations such that the cylindrical insert moved in the S-I direction (left) and center of the tumor inside the insert was aligned with the beam isocenter (right). 
During all irradiations, film and RADPOS were placed inside the lung insert (Figure 6.5) to measure the point dose at the center of the tumor (film and RADPOS) as well as the dose profile (film) along the motion path of the tumor in the S-I direction. The RADPOS detector used for these measurements was RADPOS \#1 from Table 6.2 with total dosimetric uncertainty equal to $1.4 \%$.

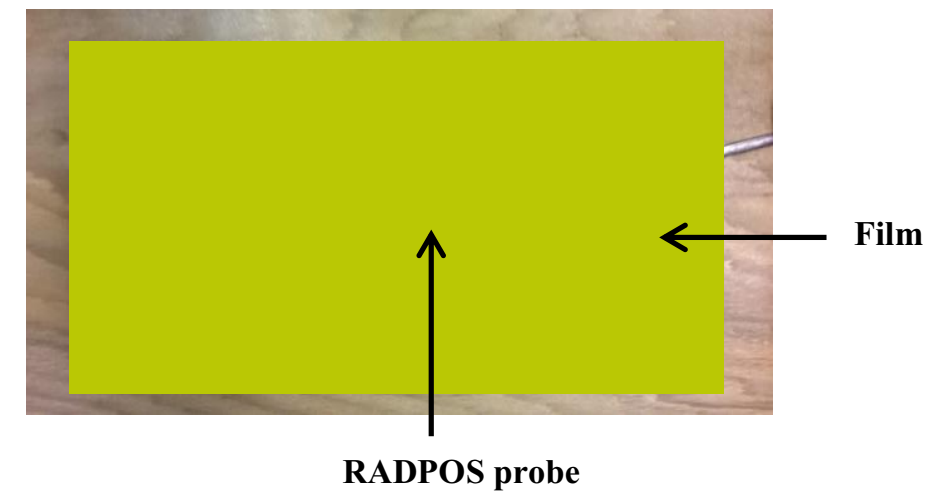

Figure 6.5 Film and RADPOS inside the lung insert of the Quasar phantom. RADPOS was placed inside a special groove, and film was taped on top of the RADPOS probe.

For irradiations with breathing motion, the RADPOS positioning sensor was used to record the motion at a temporal resolution of $100 \mathrm{~ms}$. Prior to irradiation, the computer clock times of both the linac and RADPOS computers were synchronized using the network time protocol (NTP). This was necessary to ensure synchronization of the beam delivery data in the log files with the phantom motion recorded with RADPOS. The precision of this synchronization is within $1 \mathrm{~ms}$. 


\subsubsection{Deformable phantom}

\subsubsection{CT acquisition}

For the deformable phantom with DC motor, 4DCT images were acquired during a sinusoidal motion (3.3 s period and $4 \mathrm{~cm}$ P-P piston amplitude) at an image resolution of $0.05 \times 0.05 \times 0.2 \mathrm{~cm}^{3}$ and an image matrix of $512 \times 512 \times 226$. Pitch values and gantry rotation times were equal to 0.1 and $0.5 \mathrm{~s}$ respectively. Respiratory signal was recorded using the bellows and was binned into 10 phases. Acquiring 3DCT scans was not possible with this phantom due to the non-reproducibility of the compressed state of the phantom (i.e. to stop the piston in the desired location).

For the programmable deformable phantom, two sets of 3DCT images of the phantom, in uncompressed and compressed states, were acquired. This also helped to avoid the blurring effect caused by 4DCT scans. The diaphragm displacement for the compressed state was $3 \mathrm{~cm}$, which corresponds to the maximum P-P amplitude used for measurements. These states correspond to EOI and EOE states of the phantom, respectively. The resulting resolution and matrix size of the 3 DCT images were $0.05 \times 0.05 \times 0.2 \mathrm{~cm}^{3}$ and $512 \times 512 \times 184$. Pitch values and the gantry rotation times for the scans were 0.938 and $0.75 \mathrm{~s}$, respectively.

\subsubsection{Image registration}

Deformable image registrations were performed in Velocity AI 3.2.0 (Varian Medical Systems, Palo Alto, CA, USA). The algorithm utilized by Velocity for deformable registration is a modified $\mathrm{B}$-spline algorithm ${ }^{170}$ which uses mutual information to evaluate image matching. 
The EOI and EOE scans were chosen as primary (reference) and secondary volumes, respectively. The beads were contoured as structures in both images and the structure-guided deformable registration in Velocity was used to register the EOE to the EOI image. Since CT scans of the primary and secondary images were already aligned by their DICOM coordinate systems, it was not required to perform a rigid registration before the deformable registration. The DVF was exported in binary data format (BDF) from Velocity and converted to the appropriate format for 4D MC simulations using an in-house Python code. To evaluate the accuracy of registrations, target registration errors for each landmark (i.e. Lucite beads) as well as the three RADPOS detectors were calculated in Velocity by identifying the matching bead locations in the primary and secondary images. The target registration error is defined as the distance between actual position of the landmark on the primary (EOI) image and the transformed location on the deformed secondary (EOE) image.

\subsubsection{Treatment plans}

Static and VMAT treatment plans for $6 \mathrm{MV}$ photon beams from an Elekta Infinity linac were created on the EOI scans of the phantom in Monaco V.5.11.01. Both plans delivered $100 \mathrm{cGy}$ to the center of the tumor that was contoured as the GTV with no margins added to compensate for motion.

The static treatment plan consisted of a single $3 \times 3 \mathrm{~cm}^{2}$ square field. The VMAT plan consisted of 64 control points that delivered a full arc starting and ending at $180^{\circ}$ with an average angular spacing equal to $5.6^{\circ}$. The GTV was covered by the $80 \%$ and $90 \%$ isodose lines on the static and VMAT plans, respectively. The dose calculation settings in 
Monaco were identical to those described in Section 6.2.2.3. The corresponding Monaco dose distributions are shown in Figures 6.6 and 6.7.

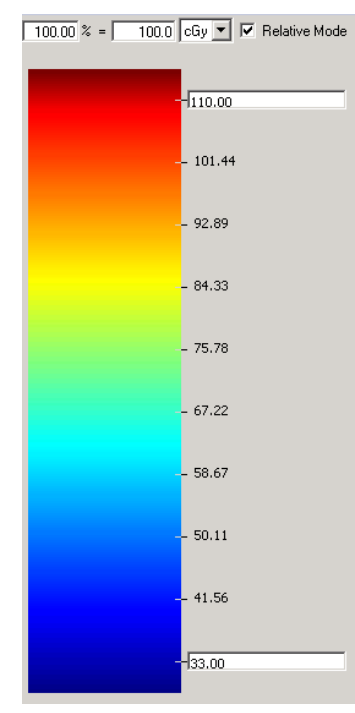

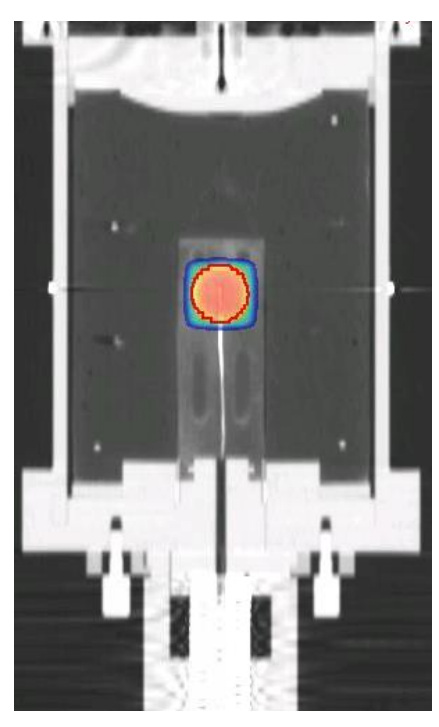

(a)

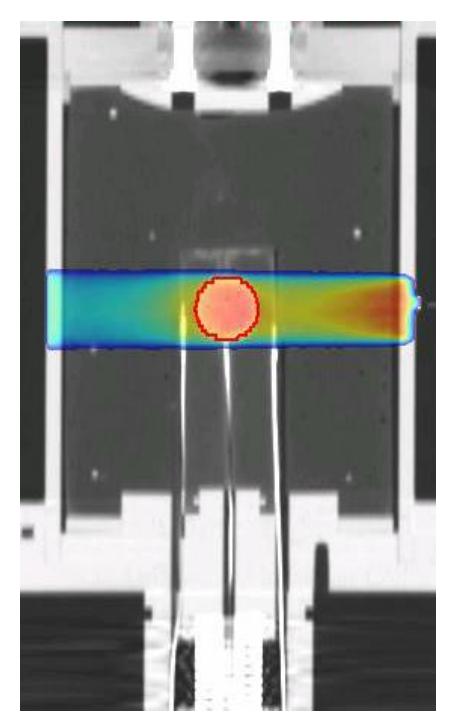

(b)

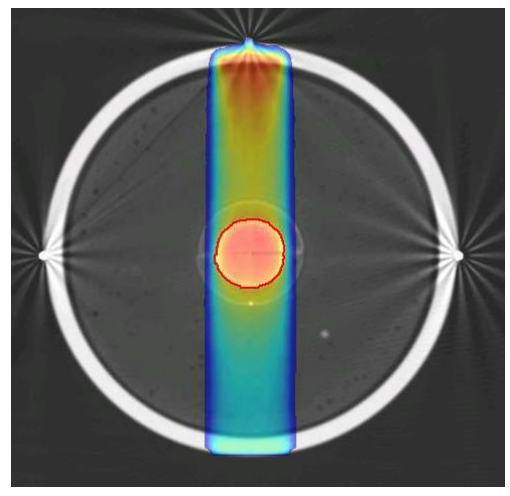

(c)

Figure 6.6 Static $3 \times 3 \mathrm{~cm}^{2}$ square plan: dose distribution from Monaco on (a) coronal, (b) sagittal and (c) axial planes. 


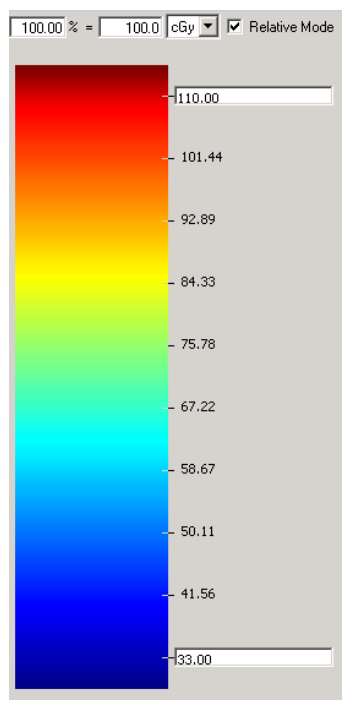

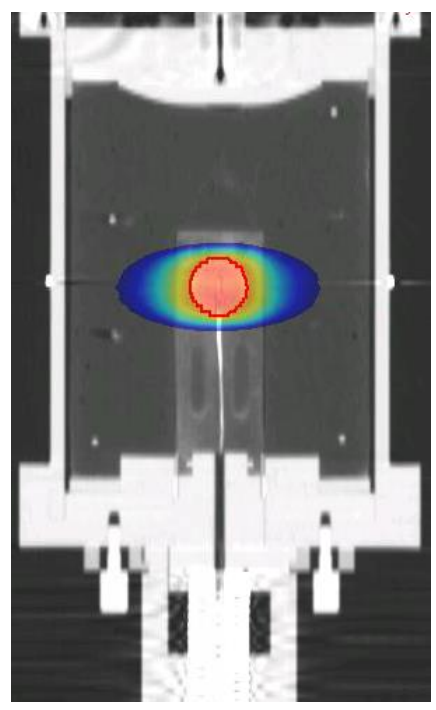

(a)

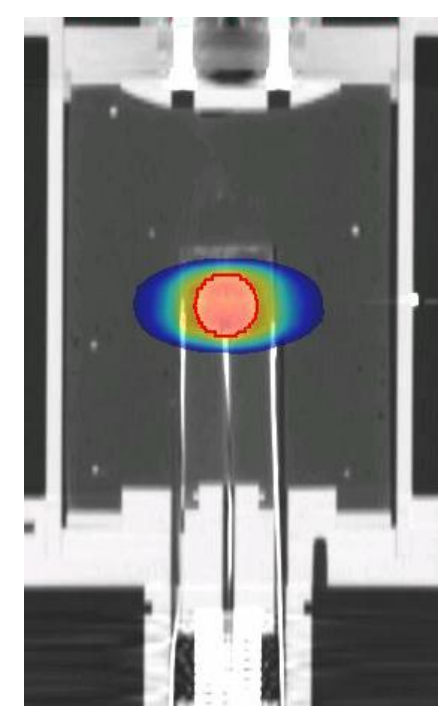

(b)

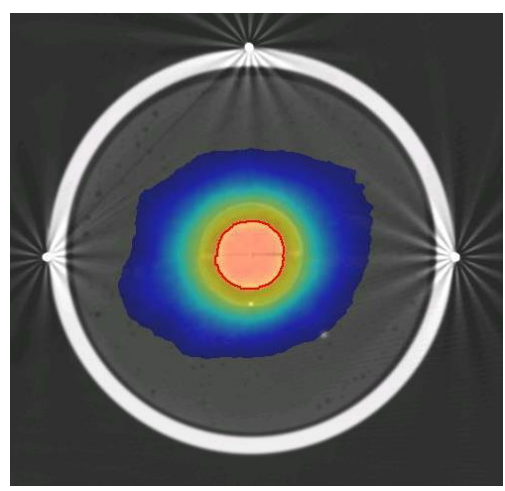

(c)

Figure 6.7 VMAT plan: dose distribution from Monaco on (a) coronal, (b) sagittal and (c) axial planes. 


\subsubsection{Phantom irradiations}

Treatment plans were exported as explained in Section 6.2.2.4 and deliveries to the phantom were performed. The static $3 \times 3 \mathrm{~cm}^{2}$ field plan delivered $110.4 \mathrm{MU}$ at a nominal dose rate of $600 \mathrm{MU} / \mathrm{min}$. The VMAT plan delivered 115.9 MU at a varying dose rate. Similar to the Quasar phantom irradiations, machine delivery log files were recorded at a temporal resolution of $40 \mathrm{~ms}$.

On the deformable phantom with DC motor, two sets of irradiations were performed on stationary (no motion) and three sets on breathing (sinusoidal motion: $3.3 \mathrm{~s}$ period, $4 \mathrm{~cm}$ P-P motion of piston) states of the phantom for each plan delivery. These same irradiations were repeated (two sets for each state of the phantom) on the deformable phantom with programmable motor to check the consistency of the results. On the programmable phantom the piston was moved at a P-P amplitude of $3 \mathrm{~cm}$. These P-P amplitudes result in a P-P motion of approximately $1.35 \mathrm{~cm}$ at the center of tumor which is reproducible within $1 \mathrm{~mm}$. In addition, the three respiratory motion profiles shown in Figure 5.9 were programmed and deliveries were repeated three times for each treatment plan. To study the sensitivity of the delivered dose to the starting phase of the motion, the three deliveries for the first (Figure 5.9 (a), typical) and second (Figure 5.9 (b), highly irregular) respiratory profiles were repeated with the beam turned on, at approximately 0 , 40 and $90 \mathrm{~s}$ after starting the phantom motion. The motion traces were recorded at a temporal resolution of $100 \mathrm{~ms}$ during deliveries using RADPOS system and the time synchronization between the beam-on and phantom motion was accomplished according to the procedure explained in Section 6.2.2.4. 
For all irradiations the phantom was placed on the couch so that the diaphragm was in the superior side (Figure 6.8) and the center of the tumor (i.e. plan isocenter) was aligned with the beam isocenter.

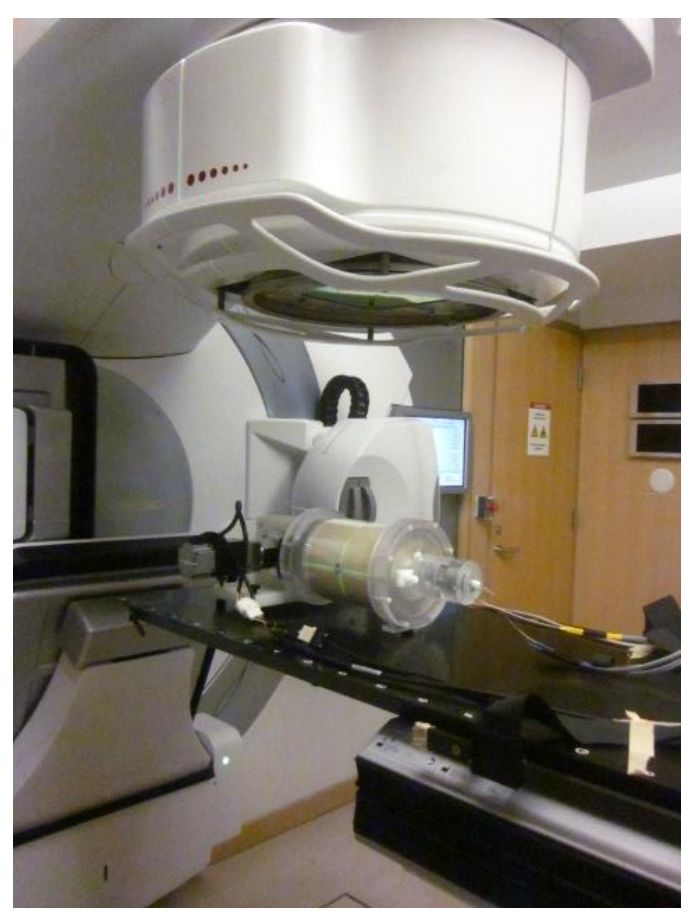

Figure 6.8 Setup for deformable phantom irradiations such that the piston was in the superior side of the couch and center of the tumor inside the phantom was aligned with the beam isocenter.

Point doses inside and outside the plug were measured using calibrated RADPOS detectors that were fixed into special grooves (Figure 5.3). Calibrated EBT3 film strips were taped on top of the RADPOS probe inside the plug to measure the dose profile in the S-I direction. Film and RADPOS inside the plug are shown in Figure 6.9. The RADPOS detectors used for these measurements included RADPOS \#2 (top), RADPOS \#3 (center) and RADPOS \#4 (bottom) from Table 6.2 with total dosimetric uncertainties of approximately $2.2 \%$ and $2.4 \%$ (center and top). 


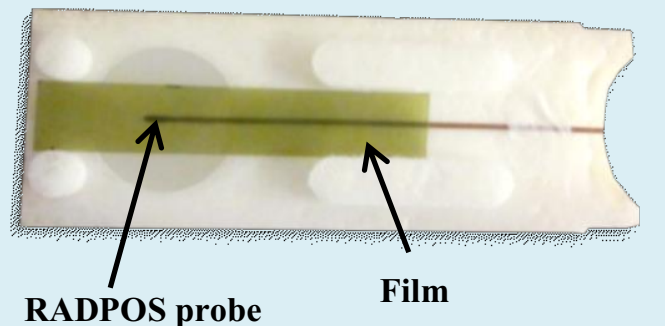

Figure 6.9 Film and RADPOS inside the plug to measure point dose (tumor center) and dose profile along the S-I direction. RADPOS is fixed inside an embedded groove and film is taped on top of RADPOS probe.

\subsubsection{Monte Carlo simulations}

The voxelized dose calculation geometry (egsphant file) was generated from the resampled 3DCT and 4DCT scans. For the Quasar phantom, the resampling was performed on the images of the phantom at the zero-amplitude position to achieve a resolution of $0.125 \times 0.125 \times 0.2 \mathrm{~cm}^{3}$. For the deformable phantom, images of EOI were resampled to a resolution of $0.2 \times 0.2 \times 0.2 \mathrm{~cm}^{3}$. Assignment of voxel densities followed the approach explained in Section 3.4 to enable direct calculation of $\mathrm{D}_{\mathrm{w}}$ in $\mathrm{MC}$ simulations to be compared against film and RADPOS measurements.

Delivery log files were converted into input files for MC simulations using an inhouse Python script. Source 21 of DOSXYZnrc was used to simulate the BEAMnrc linac model for the simulations performed in this work. Details on this source were previously presented in Section 3.3. To model the phantom motion and deformation during 4DdefDOSXYZnrc simulations, manually generated displacement vectors (Quasar phantom) or a DVF exported from Velocity (deformable phantom) along with the 
respiratory motion trace recorded with RADPOS (tumor center) during irradiations were used. The RADPOS motion was resampled to match the temporal resolution of the $\log$ files. It was then normalized to the maximum displacement to generate the deformation vector scaling factor as explained in Section 3.3. These scaling factors ranged from -1 to 1 for the Quasar phantom and from 0 (EOI) to 1 (EOE) for the deformable phantom.

On the Quasar phantom, $2.5 \times 10^{8}$ and $4.5 \times 10^{8}$ histories were simulated on the stationary and moving geometries, respectively, requiring approximately $20-25$ and 50-55 CPU core hours per calculation. On the deforming phantom, $1.5 \times 10^{8}$ and $3.0 \times 10^{8}$ histories were simulated requiring approximately $12-15$ and 31-35 CPU core hours per calculation.

\subsubsection{Comparison metrics}

Point dose comparisons between MC simulations and measurements at the center of the tumor (film and RADPOS) are quoted as percentage of the measured dose value at the point of measurement. For the deformable phantom, similar comparisons were performed for point dose values between MC simulations and RADPOS measurements at the top and bottom surfaces of the plug.

Dose profiles along the motion path of the phantom (S-I direction) from MC simulations were compared against film measurements. To evaluate dose profiles from simulations, a $1 \mathrm{D}$ global gamma analysis with a $2 \%$ dose-difference and $2 \mathrm{~mm}$ distanceto-agreement criteria with film as the reference was utilized. The dose threshold used for gamma analysis was set to be 5\% (Quasar and deformable phantom) and 50\% (Quasar phantom) of the evaluated maximum dose $\left(\mathrm{D}_{\max }\right)$. Measurement of the profiles on the deformable phantom was limited by the deformations in the superior side of the phantom 
where the piston is placed. Moreover, deformations of the phantom introduced a constraint on using wider film pieces to acquire 2D dose distribution. For the Quasar phantom, 2D dose distributions from film and $\mathrm{MC}$ simulations were extracted and compared using a 2D global gamma analysis with the same parameters as in 1D gamma analysis. To match the dose grid resolution of film with the one from MC simulations $(2 \mathrm{~mm})$, a moving average filter was applied to the film readings.

\subsection{Results}

\subsubsection{Validation of image registration}

The accuracy of the deformable image registrations was evaluated both visually (Figure 6.10) and quantitatively (Table 6.3). Mean values of target registration errors with their standard deviations are shown in Table 6.3 for A-P, L-R and S-I directions. The overall $3 \mathrm{D}$ registration error is presented in the table as well. The $3 \mathrm{D}$ registration error for each landmark (i.e. Lucite beads) as well as each RADPOS detector is calculated by adding errors in all three directions in quadrature. The minimum, maximum and mean 3D error values for the three RADPOS detectors (which were not used to guide the deformable registration) were $0.06,0.13$ and $0.01 \pm 0.04 \mathrm{~cm}$, respectively. Overall and for all landmarks used to evaluate registration, the maximum and minimum 3D error values were measured to be 0.18 and $0.04 \mathrm{~cm}$, respectively. The limiting factor in achieving a better registration accuracy was the resolution of the CT image slices which was $0.2 \mathrm{~cm}$. 
Table 6.3 Mean registration errors with their standard deviations in A-P, L-R, S-I and 3D.

\begin{tabular}{cc}
\hline \hline Direction of motion & Mean registration error $(\mathrm{cm})$ \\
\hline & \\
A-P & $0.05 \pm 0.03$ \\
L-R & $0.04 \pm 0.03$ \\
S-I & $0.08 \pm 0.05$ \\
3D & $0.12 \pm 0.04$ \\
\hline \hline
\end{tabular}

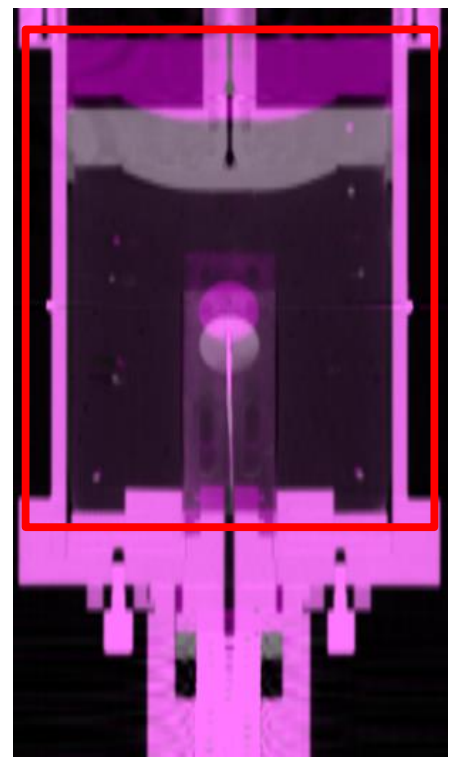

(a)

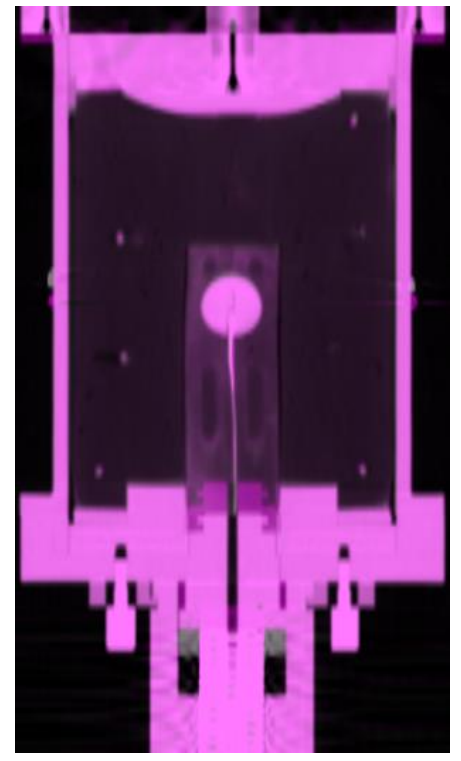

(b)

Figure 6.10 Coronal view of the phantom in Velocity: (a) non-deformed EOE (gray) overlaid on EOI (pink), (b) deformed EOE overlaid on EOI. The red rectangular area shows the selected region of interest (ROI) for image registration.

Figures 6.10 (a) and 6.10 (b) show the overlaid EOI and EOE images before and after registration. The red rectangle shown in Figure 6.10 (a) represents the region of interest (ROI) used to contain the regions of the phantom included in image registration. 
The size and location of this ROI was found to have an important effect on the quality of registration. The difference map shown in Figure 6.11 (a) presents the differences between the deformed EOE image from the EOI. Regions in light gray present the lowest differences between the deformed EOE and EOI images. In Figure 6.11 (b) the Jacobian map of the registration is representative of how voxels change in size once deformations are applied. In this colormap, green regions show a 1-to-1 voxel size between deformed EOE and EOI images. Blue and red regions present shrinkage (reduced voxel size) and growth (enlarged voxel size) on the other hand.

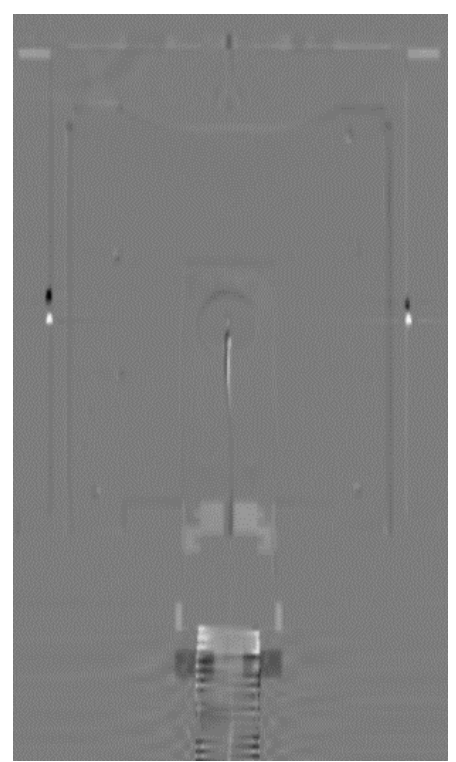

(a)

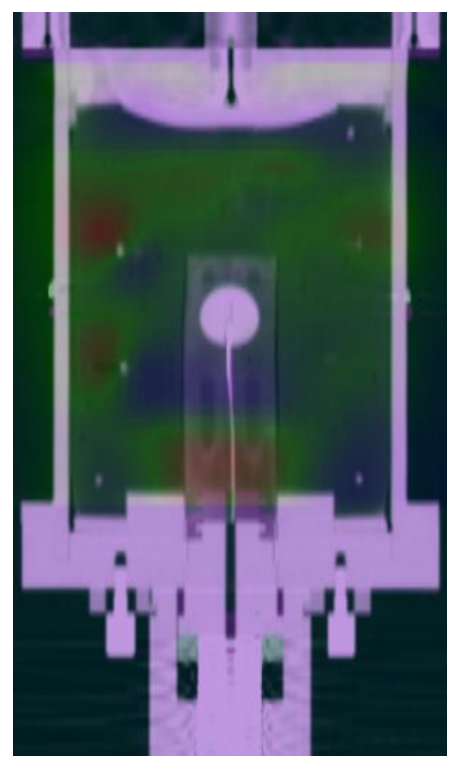

(b)

Figure 6.11. Visual evaluation of the deformable image registration on coronal view: (a) deformed EOE subtracted from EOI and (b) Jacobian of the registration. 


\subsubsection{Stationary phantom comparisons}

\subsubsection{Quasar phantom}

Figures 6.11 and 6.12 show the comparison of dose profiles, 2D dose distributions and gamma comparison maps $\left(50 \% \mathrm{D}_{\max }\right.$ dose threshold) for $\mathrm{MC}$ simulations against film measurements for the $4 \times 4 \mathrm{~cm}^{2}$ and VMAT plan deliveries on the stationary state of the Quasar phantom. Overall, a good agreement was observed between measurements and simulations. The passing rates for the $1 \mathrm{D}$ and $2 \mathrm{D}$ gamma comparisons $(2 \% / 2 \mathrm{~mm})$ are presented in Table 6.4. In addition, measured and simulated dose values at the center of the tumor are shown in Table 6.5. The reported uncertainties are experimental and statistical uncertainties corresponding to measurements and MC simulations, respectively. The agreement between MC simulations and film measurements was found to be slightly over 1\%. RADPOS and MC simulations on the other hand, had an agreement of approximately $2.3 \%$ which is within the $2 \sigma$ of experimental uncertainties $(2.8 \%)$. 

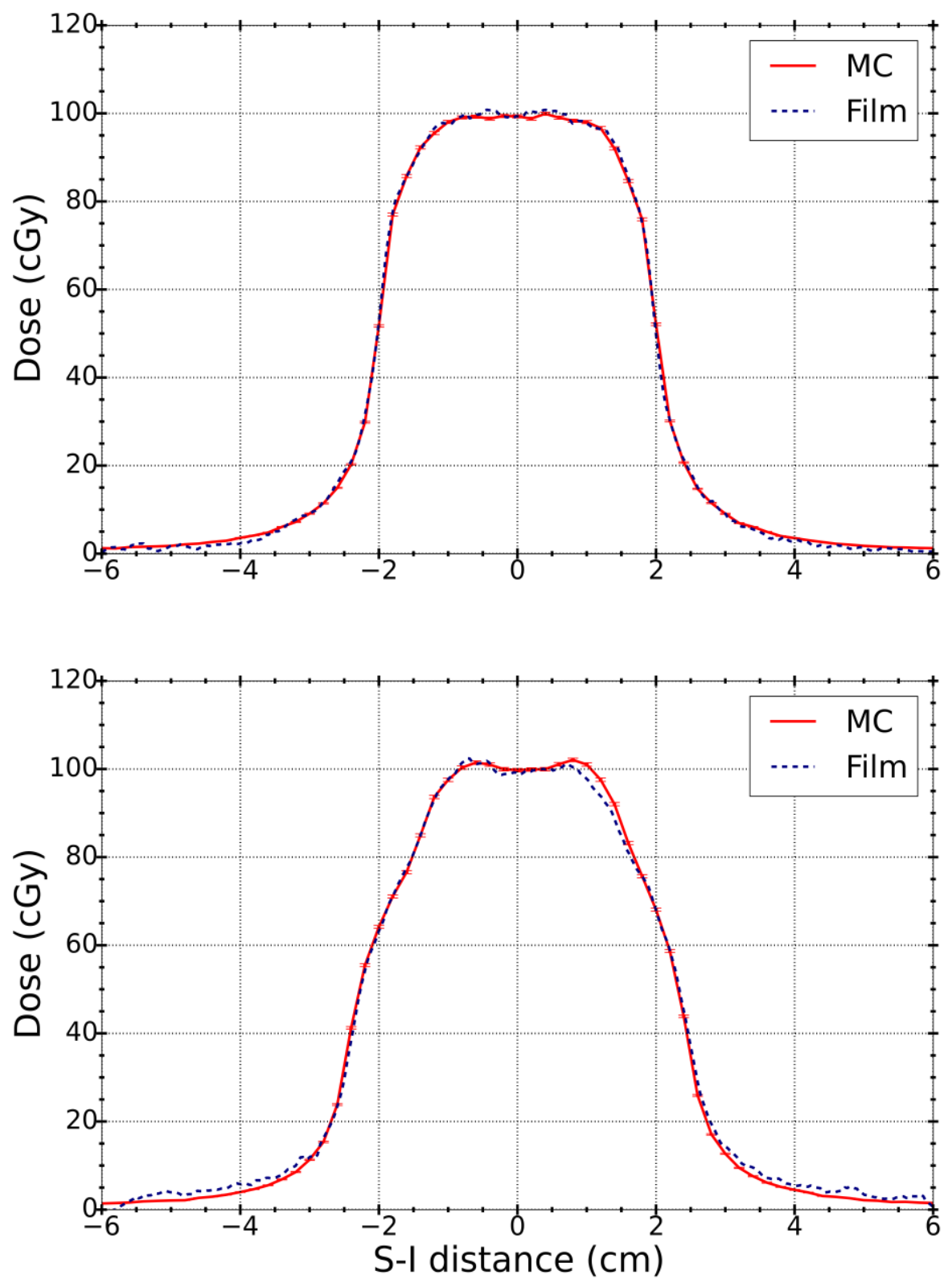

Figure 6.12 Comparison of dose profiles for $4 \times 4 \mathrm{~cm}^{2}$ (top) and VMAT (bottom) beam deliveries on the stationary Quasar phantom along the S-I direction. 

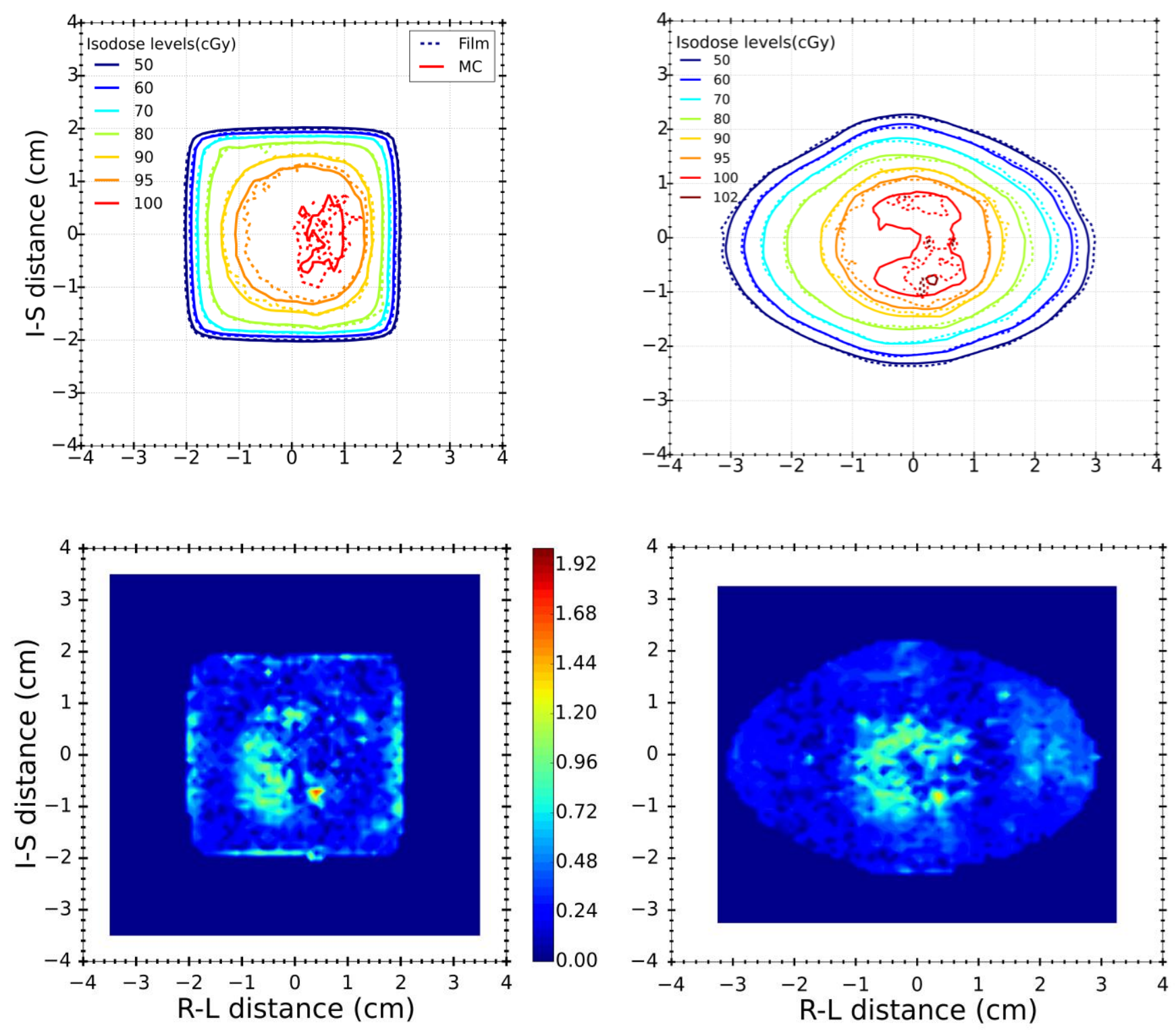

Figure 6.13 Comparison of 2D dose distributions (top row) and gamma comparison maps (bottom row) from film measurements and MC simulations for $4 \times 4 \mathrm{~cm}^{2}$ (left) and VMAT (right) beam deliveries on the stationary Quasar phantom. Gamma comparison maps were generated using a threshold of $50 \% \mathrm{D}_{\text {max. }}$ 
Table 6.4 Passing rates of $1 \mathrm{D}$ and 2D gamma comparisons of $2 \% / 2 \mathrm{~mm}$ criterion for MC simulations against film measurements on the stationary Quasar phantom.

\begin{tabular}{|c|c|c|c|c|}
\hline \multirow[b]{3}{*}{ Plan } & \multicolumn{4}{|c|}{$2 \% / 2 \mathrm{~mm}$ gamma passing rate $(\%)$} \\
\hline & \multicolumn{2}{|c|}{ Threshold $=5 \% \mathrm{D}_{\max }$} & \multicolumn{2}{|c|}{ Threshold $=50 \% \mathrm{D}_{\max }$} \\
\hline & $1 \mathrm{D}$ & $2 \mathrm{D}$ & $1 \mathrm{D}$ & $2 \mathrm{D}$ \\
\hline Static $4 \times 4 \mathrm{~cm}^{2}$ & 100.0 & 99.5 & 99.1 & 98.9 \\
\hline VMAT & 100.0 & 97.9 & 100.0 & 99.5 \\
\hline
\end{tabular}

Table 6.5 Dose values at the center of tumor from measurements with film and RADPOS as well as MC simulations on the stationary Quasar phantom.

\begin{tabular}{|c|c|c|c|}
\hline \multirow[b]{3}{*}{ Plan } & \multicolumn{3}{|c|}{ Dose (cGy) } \\
\hline & \multirow[b]{2}{*}{$\mathrm{MC}$} & \multicolumn{2}{|c|}{ Measurements } \\
\hline & & Film & RADPOS \\
\hline Static $4 \times 4 \mathrm{~cm}^{2}$ & $99.3 \pm 0.4 \%$ & $100.5 \pm 2.3 \%$ & $101.6 \pm 1.4 \%$ \\
\hline VMAT & $99.8 \pm 0.4 \%$ & $99.0 \pm 2.3 \%$ & $98.4 \pm 1.4 \%$ \\
\hline
\end{tabular}




\subsubsection{Deformable phantom}

The comparison of dose profiles from MC simulations and film measurements for the $3 \times 3 \mathrm{~cm}^{2}$ and VMAT plan deliveries on the stationary phantom are shown in Figure 6.13. Measured and simulated profiles were in very good agreement. All dose points for the VMAT plan delivery passed the $1 \mathrm{D}$ gamma comparison with $2 \% / 2 \mathrm{~mm}$ criteria. For the $3 \times 3 \mathrm{~cm}^{2}$ square field, the passing rate was $99.2 \%$. The average gamma values from all irradiation sets were calculated to be $(99.3 \pm 1.2) \%$ and $(98.4 \pm 2.6) \%$ for the VMAT and $3 \times 3 \mathrm{~cm}^{2}$ plan deliveries, respectively.

Dose values measured and simulated at the center of tumor are shown in Table 6.6 with their corresponding statistical and experimental uncertainties. All dose values from MC simulations were found to be approximately within $2 \%$ of film and RADPOS measurements for both plan deliveries. Comparisons of all irradiation sets also showed same level of agreement. In Table 6.7 we can see dose values for point dose measurements at the top and bottom surfaces of the plug. At the bottom surface of the plug, an agreement of approximately $2 \%$ was observed for all irradiations. At the top surface, agreements were found to be better than $4 \%$ for all irradiations. It should be noted that this dose point is located in the penumbra region of the profile which corresponds to higher dose gradient and as a result the dose reading or positional uncertainties are increased compared to the dose point at the bottom surface. The corresponding uncertainties are approximately $6 \%$ and $1 \%$ for the dose points at the top and bottom surfaces of the plug for irradiations on the stationary state of the phantom. In addition, we can see that for dose values measured with RADPOS, all comparisons lie within the $2 \sigma$ of experimental uncertainties $(\sim 5 \%)$. 

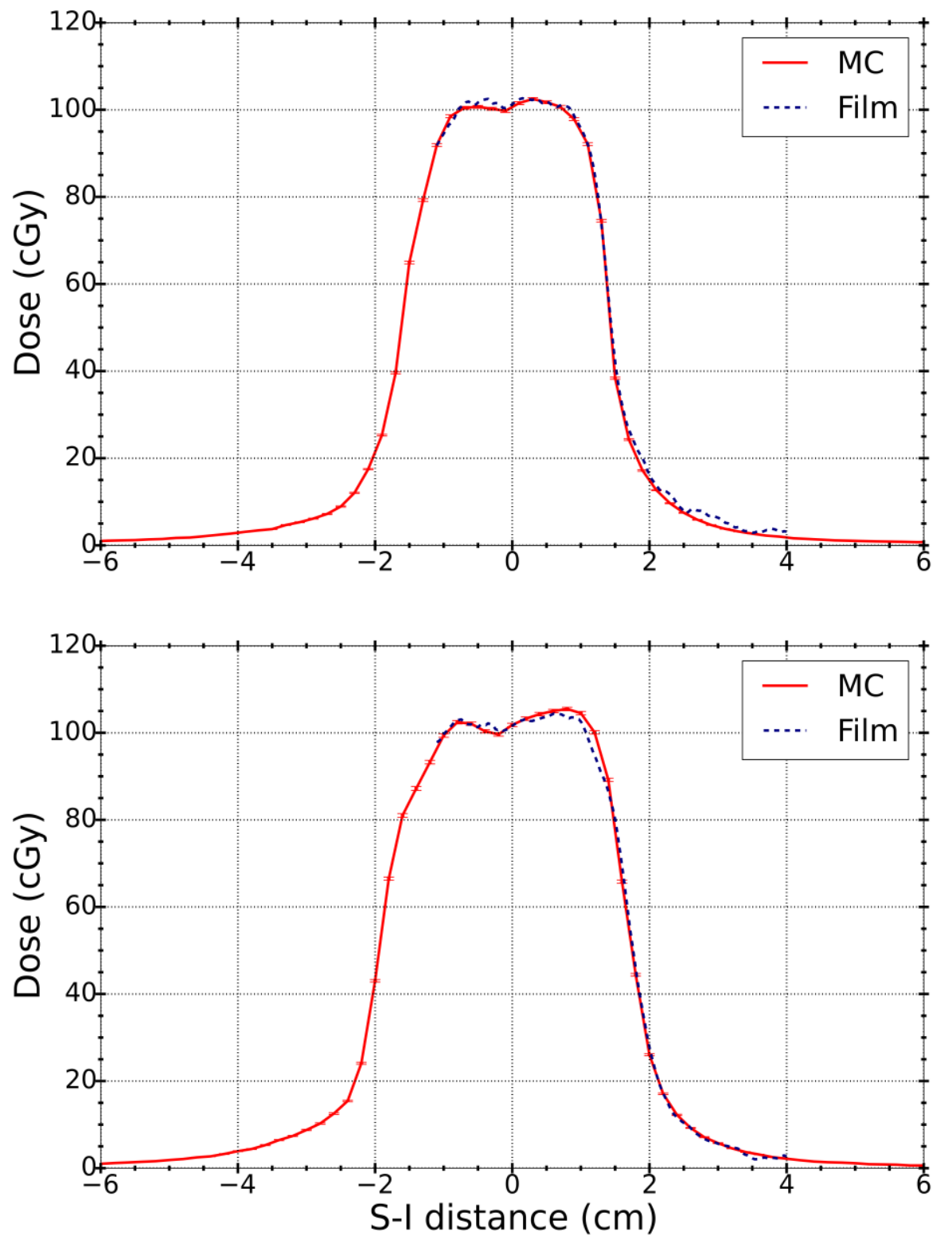

Figure 6.14 Comparison of dose profiles for $3 \times 3 \mathrm{~cm}^{2}$ (top) and VMAT (bottom) beam deliveries on the stationary deformable phantom along the S-I direction. 
Table 6.6 Dose values at the center of tumor from measurements with film and RADPOS as well as MC simulations on the stationary deformable phantom.

\begin{tabular}{|c|c|c|c|}
\hline \multirow[b]{3}{*}{ Plan } & \multicolumn{3}{|c|}{ Dose (cGy) } \\
\hline & \multirow[b]{2}{*}{$\mathrm{MC}$} & \multicolumn{2}{|c|}{ Measurements } \\
\hline & & Film & RADPOS \\
\hline Static $3 \times 3 \mathrm{~cm}^{2}$ & $100.8 \pm 0.4 \%$ & $101.2 \pm 2.3 \%$ & $99.5 \pm 2.4 \%$ \\
\hline VMAT & $101.6 \pm 0.4 \%$ & $101.6 \pm 2.3 \%$ & $99.9 \pm 2.4 \%$ \\
\hline
\end{tabular}

Table 6.7 Dose values at the top and bottom surfaces of the plug from MC simulations and RADPOS measurements on the stationary deformable phantom. The top surface RADPOS was placed at an offset position of $1 \mathrm{~cm}$ from the tumor center.

\begin{tabular}{|c|c|c|c|c|}
\hline \multirow[b]{3}{*}{ Plan } & \multicolumn{4}{|c|}{ Dose (cGy) } \\
\hline & \multicolumn{2}{|c|}{ Top surface } & \multicolumn{2}{|c|}{ Bottom surface } \\
\hline & $\mathrm{MC}$ & RADPOS & $\mathrm{MC}$ & RADPOS \\
\hline Static $3 \times 3 \mathrm{~cm}^{2}$ & $77.3 \pm 0.4 \%$ & $80.0 \pm 2.2 \%$ & $84.3 \pm 0.4 \%$ & $83.0 \pm 2.4 \%$ \\
\hline VMAT & $75.4 \pm 0.4 \%$ & $76.6 \pm 2.2 \%$ & $85.3 \pm 0.4 \%$ & $83.1 \pm 2.4 \%$ \\
\hline
\end{tabular}




\subsubsection{Moving and deforming phantom comparisons}

\subsubsection{Quasar Phantom}

Comparison of dose profiles, 2D dose distributions and gamma comparison maps $50 \% \mathrm{D}_{\max }$ dose threshold) for MC simulations against film measurements for the $4 \times 4 \mathrm{~cm}^{2}$ and VMAT plan deliveries on the breathing Quasar phantom are shown in Figures 6.14 and 6.15. In addition, $1 \mathrm{D}$ and $2 \mathrm{D}$ passing rates for the $2 \% / 2 \mathrm{~mm}$ gamma comparisons as well as point dose values at the center of the tumor are shown in Tables 6.8 and 6.9, respectively. Dose values from MC simulations and film measurements were found to agree within $1.0 \%$ for both plan deliveries. RADPOS measurements and MC simulations

agreed within $2.5 \%$ for both plan deliveries which is within $2 \sigma$ of experimental uncertainties $(2.8 \%)$. 

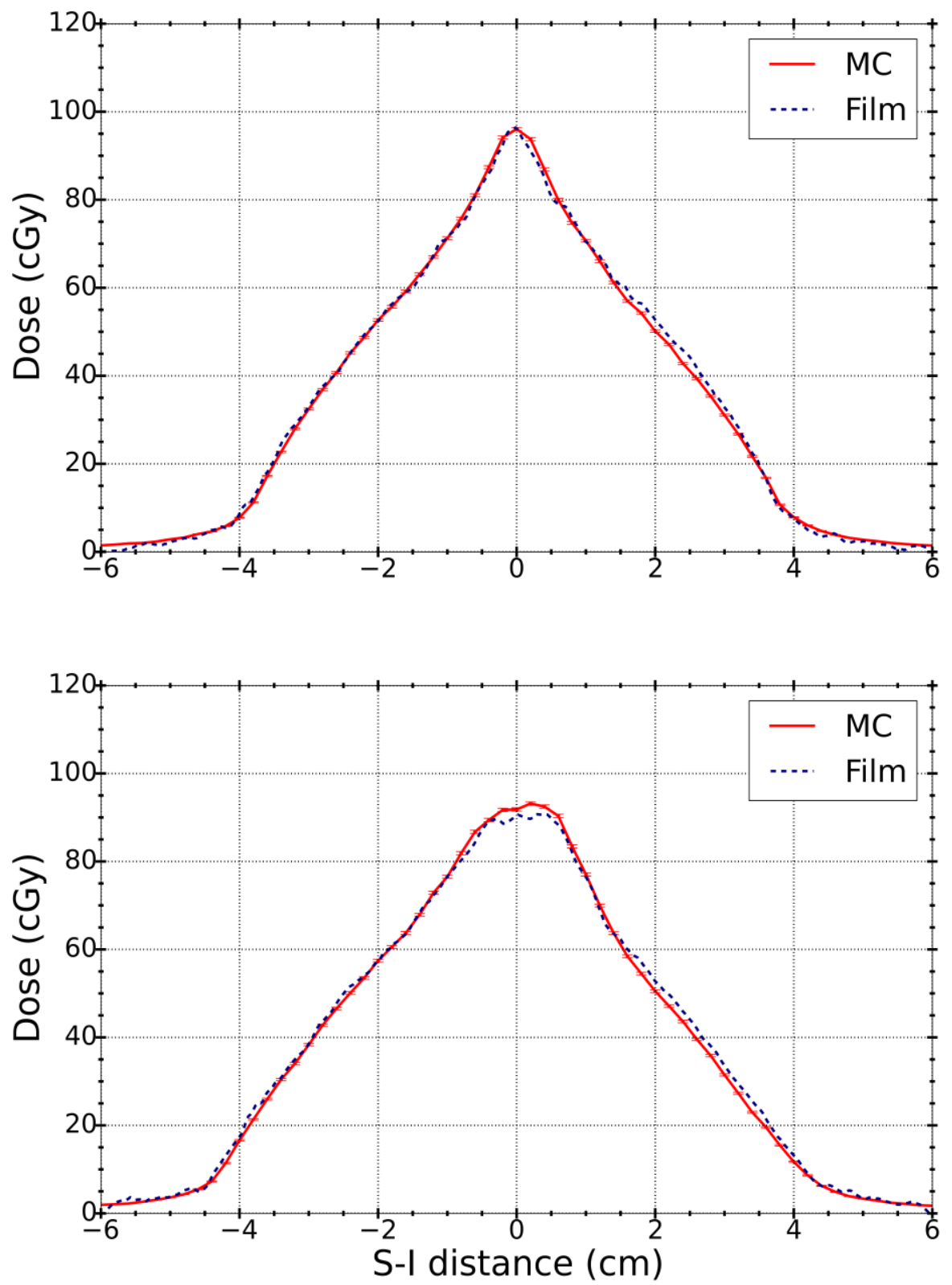

Figure 6.15 Comparison of dose profiles for $4 \times 4 \mathrm{~cm}^{2}$ (top) and VMAT (bottom) beam deliveries on the breathing Quasar phantom along the S-I direction. 

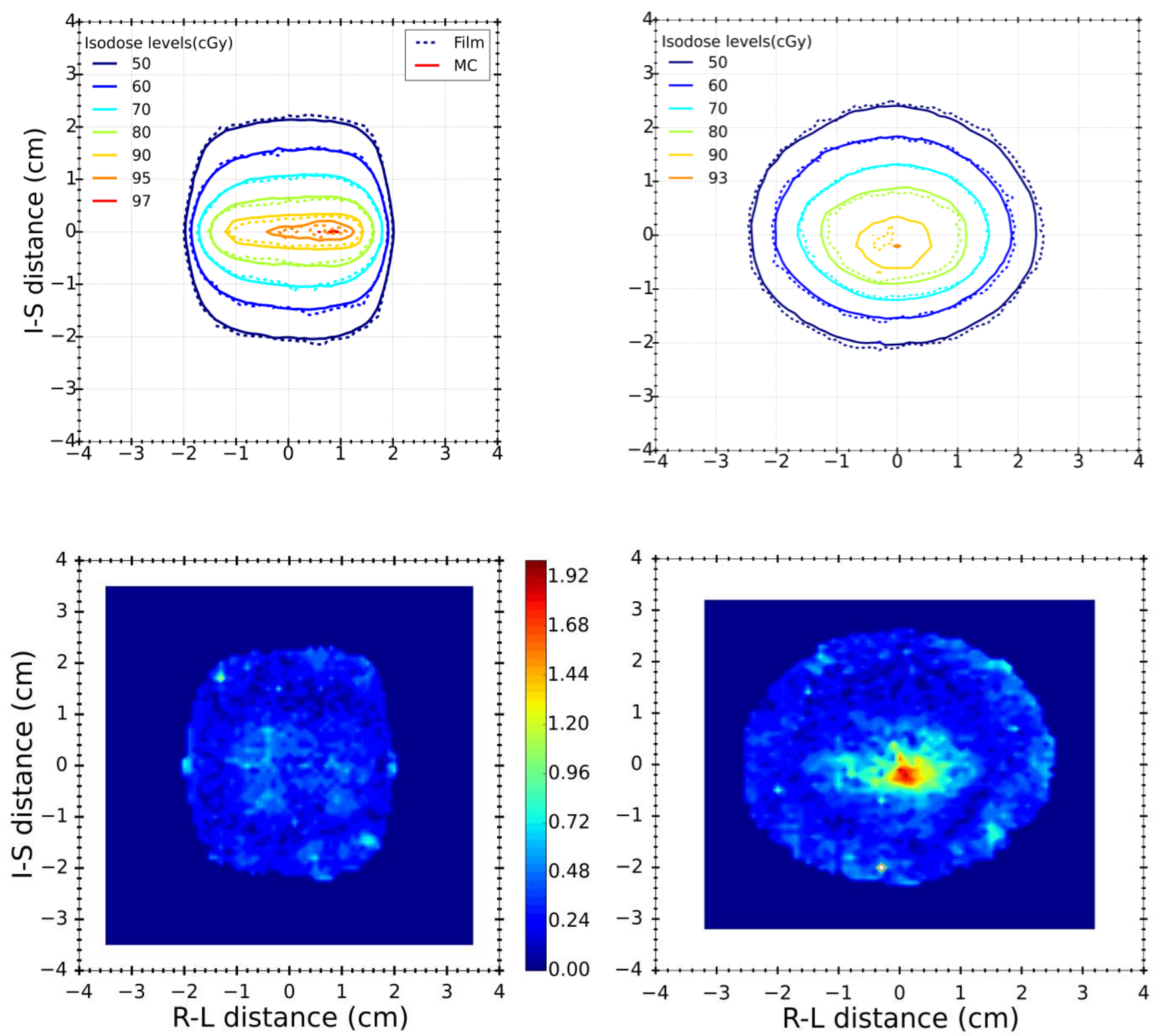

Figure 6.16 Comparison of 2D dose distributions (top row) and gamma comparison maps (bottom row) from film measurements and MC simulations for $4 \times 4 \mathrm{~cm}^{2}$ (left) and VMAT (right) beam deliveries on the breathing Quasar phantom. Gamma comparison maps were generated using a threshold of $50 \% \mathrm{D}_{\max }$. 
Table 6.8 Passing rates of 1D and 2D gamma comparisons of $2 \% / 2 \mathrm{~mm}$ criterion for MC simulations against film measurements on the breathing Quasar phantom.

\begin{tabular}{|c|c|c|c|c|}
\hline \multirow[b]{3}{*}{ Plan } & \multicolumn{4}{|c|}{$2 \% / 2 \mathrm{~mm}$ gamma passing rate $(\%)$} \\
\hline & \multicolumn{2}{|c|}{ Threshold $=5 \% \mathrm{D}_{\max }$} & \multicolumn{2}{|c|}{ Threshold $=50 \% \mathrm{D}_{\max }$} \\
\hline & $1 \mathrm{D}$ & $2 \mathrm{D}$ & $1 \mathrm{D}$ & $2 \mathrm{D}$ \\
\hline Static $4 \times 4 \mathrm{~cm}^{2}$ & 100.0 & 100.0 & 99.1 & 100.0 \\
\hline VMAT & 96.0 & 94.6 & 94.4 & 97.4 \\
\hline
\end{tabular}

Table 6.9 Dose values at the center of tumor from measurements with film and RADPOS as well as MC simulations on the breathing Quasar phantom.

\begin{tabular}{|c|c|c|c|}
\hline \multirow[b]{3}{*}{ Plan } & \multicolumn{3}{|c|}{ Dose (cGy) } \\
\hline & \multirow[b]{2}{*}{$\mathrm{MC}$} & \multicolumn{2}{|c|}{ Measurements } \\
\hline & & Film & RADPOS \\
\hline Static $4 \times 4 \mathrm{~cm}^{2}$ & $96.1 \pm 0.4 \%$ & $96.4 \pm 2.3 \%$ & $98.4 \pm 1.4 \%$ \\
\hline VMAT & $91.9 \pm 0.4 \%$ & $91.1 \pm 2.3 \%$ & $89.6 \pm 1.4 \%$ \\
\hline
\end{tabular}




\subsubsection{Deformable phantom}

Figure 6.16 shows sample dose profiles from $\mathrm{MC}$ simulations and film measurements for sinusoidal respiratory motion on the breathing deformable phantom. Examples of dose profiles from measurements and $\mathrm{MC}$ simulations with respiratory motion profiles previously presented in Figure 5.9 are also shown in Figures 6.17-6.19. Dose profiles correspond respectively to simulations and measurements on deformable phantom moving with respiratory traces with typical motion (Figure 5.9 (a)), highly irregular motion (Figure 5.9 (b)) as well as one with a large level of hysteresis (Figure 5.9 (c)). Corresponding 1D gamma passing rates $(2 \% / 2 \mathrm{~mm})$ for the dose profiles shown in Figures 6.16-6.19 are shown in Table 6.10. In addition, average values of gamma passing rates for all sets of irradiations were calculated and are shown in this table.

Also, dose values measured and simulated at the center of tumor as well as top and bottom surfaces of the plug are shown in Tables $6.11,6.12$ and 6.13 with their corresponding statistical and experimental uncertainties. 

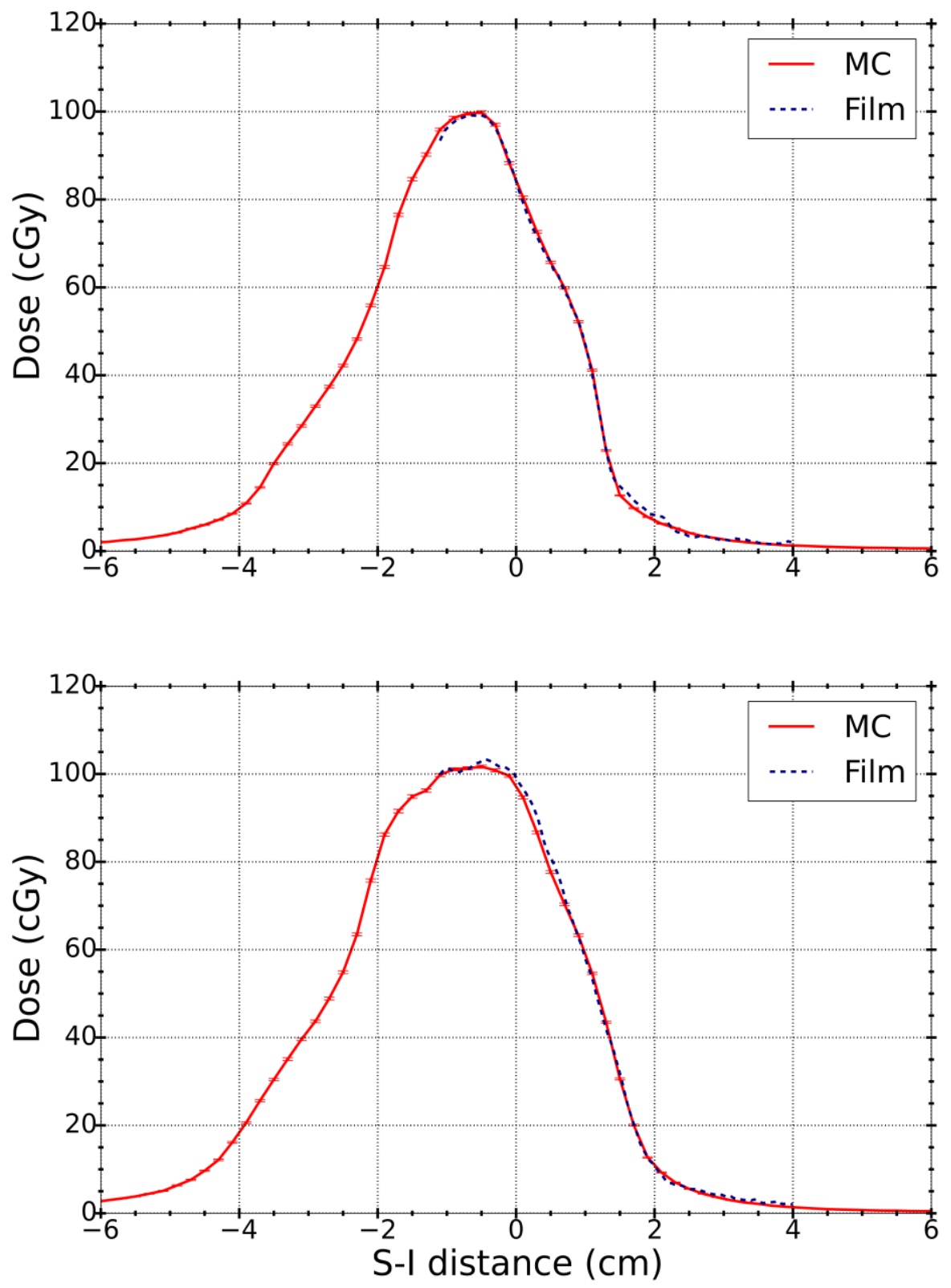

Figure 6.17 Comparison of dose profiles for $3 \times 3 \mathrm{~cm}^{2}$ (top) and VMAT (bottom) beam deliveries on the breathing deformable phantom along the S-I direction, for the sinusoidal breathing motion. 

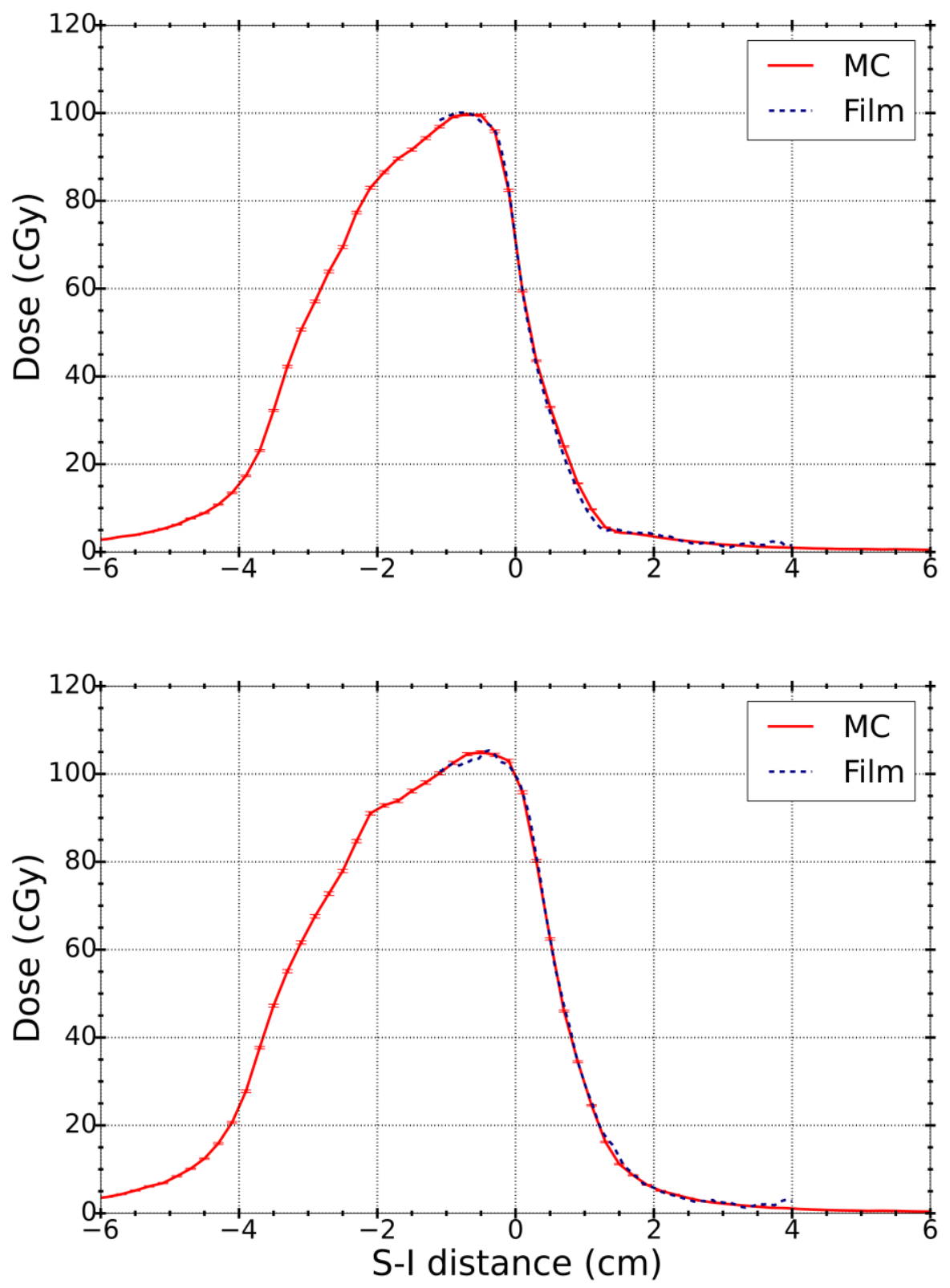

Figure 6.18 Comparison of dose profiles for $3 \times 3 \mathrm{~cm}^{2}$ (top) and VMAT (bottom) beam deliveries on the breathing deformable phantom along the S-I direction, for the typical breathing motion shown in Figure 5.9 (a). 

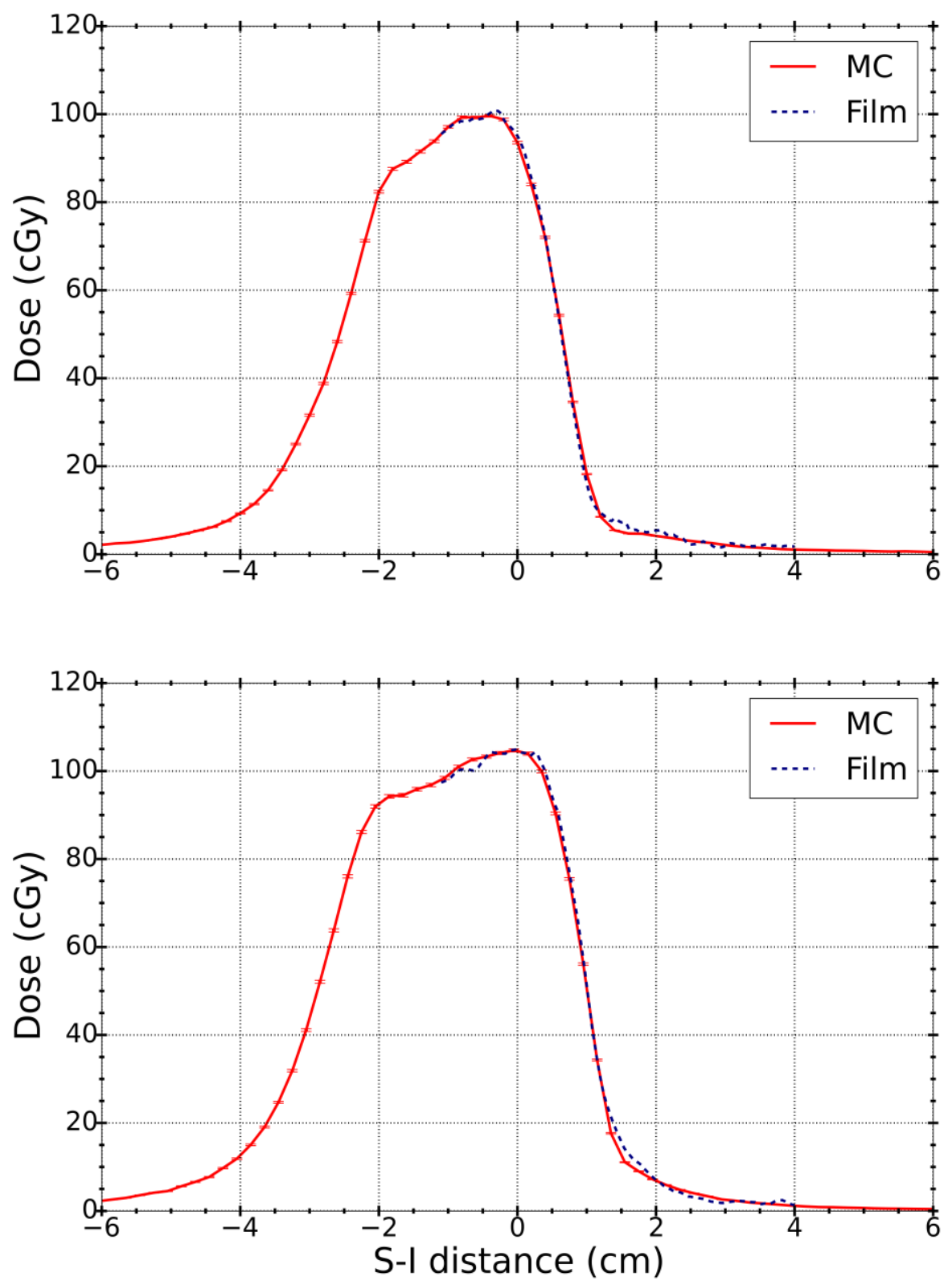

Figure 6.19 Comparison of dose profiles for $3 \times 3 \mathrm{~cm}^{2}$ (top) and VMAT (bottom) beam deliveries on the breathing deformable phantom along the $\mathrm{S}$ I direction, for the highly irregular breathing motion shown in Figure 5.9 (b). 

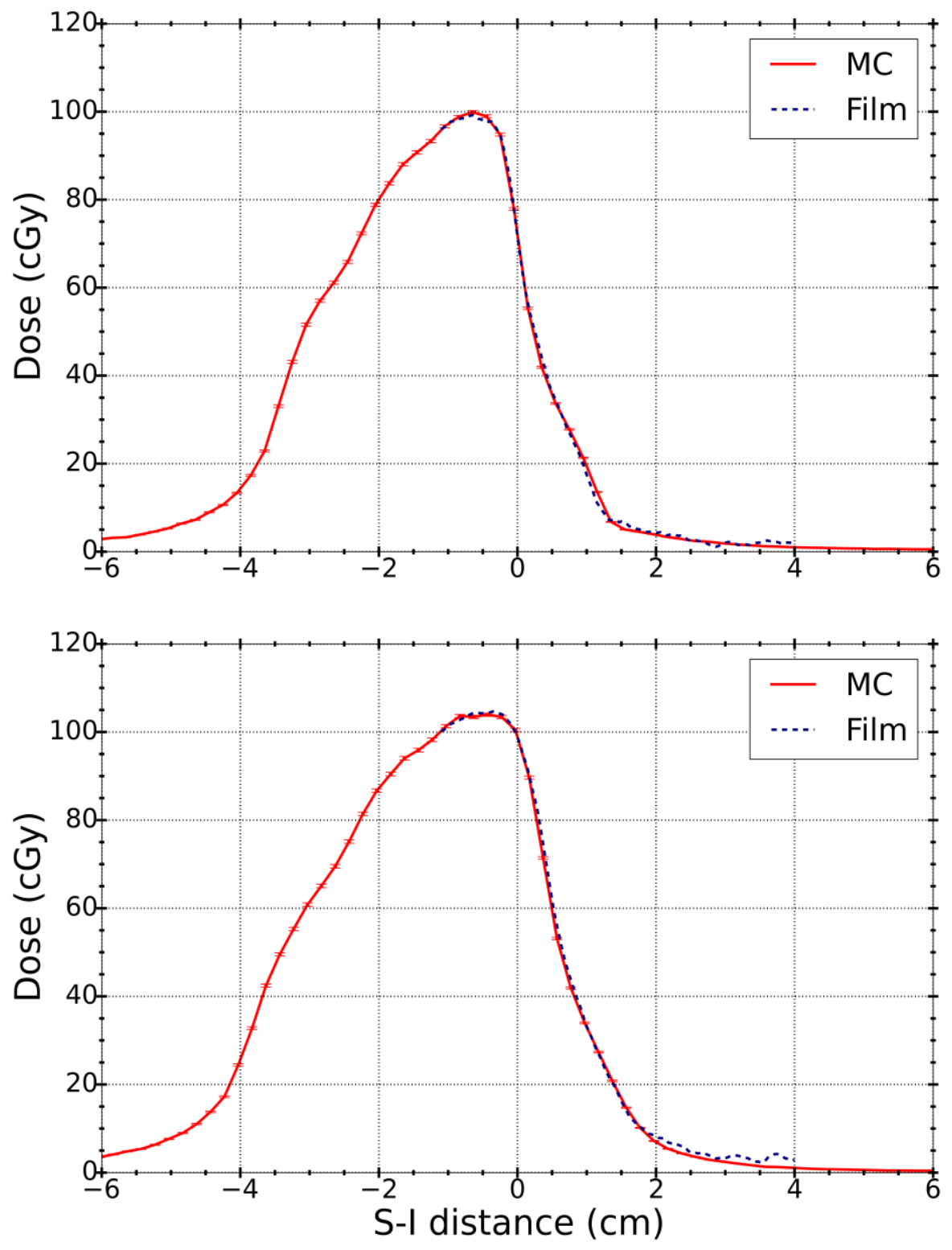

Figure 6.20 Comparison of dose profiles for $3 \times 3 \mathrm{~cm}^{2}$ (top) and VMAT (bottom) beam deliveries on the breathing deformable phantom along the S-I direction, for the breathing motion with large hysteresis between the tumor and diaphragm motion shown in Figure 5.9 (c). 
Table 6.10 Passing rates of 1D gamma comparisons of $2 \% / 2 \mathrm{~mm}$ criterion for MC simulations against film measurements on the breathing deformable phantom for sinusoidal, typical, highly irregular and large hysteresis between the tumor and diaphragm motion respiratory motion profiles. First column of gamma passing rates corresponds to the dose profiles shown in Figures 6.16-6.19. The average values from all irradiation sets are shown in the second column.

\begin{tabular}{|c|c|c|c|}
\hline \multirow[b]{2}{*}{ Plan } & \multirow[b]{2}{*}{ Motion } & \multicolumn{2}{|c|}{$2 \% / 2 \mathrm{~mm} 1 \mathrm{D}$ gamma passing rate $(\%)$} \\
\hline & & Single profile & Total \\
\hline \multirow{4}{*}{ Static $3 \times 3 \mathrm{~cm}^{2}$} & $\begin{array}{l}\text { Sinusoidal } \\
\text { (Figure } 6.16 \text { (top)) }\end{array}$ & 100.0 & $96.2 \pm 3.3$ \\
\hline & $\begin{array}{l}\text { Typical } \\
\text { (Figure } 6.17 \text { (top)) }\end{array}$ & 100.0 & $97.7 \pm 2.9$ \\
\hline & $\begin{array}{l}\text { Highly irregular } \\
\text { (Figure } 6.18 \text { (top)) }\end{array}$ & 98.9 & $98.0 \pm 2.6$ \\
\hline & $\begin{array}{l}\text { Large hysteresis } \\
\text { (Figure } 6.19 \text { (top)) }\end{array}$ & 100.0 & $99.3 \pm 1.2$ \\
\hline \multirow{4}{*}{ VMAT } & $\begin{array}{l}\text { Sinusoidal } \\
\text { (Figure } 6.16 \text { (bottom)) }\end{array}$ & 100.0 & $98.1 \pm 3.2$ \\
\hline & $\begin{array}{l}\text { Typical } \\
\text { (Figure } 6.17 \text { (bottom)) }\end{array}$ & 99.7 & $97.8 \pm 1.9$ \\
\hline & $\begin{array}{l}\text { Highly irregular } \\
\text { (Figure } 6.18 \text { (bottom)) }\end{array}$ & 98.9 & $98.1 \pm 1.3$ \\
\hline & $\begin{array}{l}\text { Large hysteresis } \\
\text { (Figure } 6.19 \text { (bottom)) }\end{array}$ & 100.0 & $97.9 \pm 1.8$ \\
\hline
\end{tabular}


Table 6.11 Dose values at the center of tumor from measurements with film and RADPOS as well as MC simulations on the breathing deformable phantom during sinusoidal, typical, highly irregular and large hysteresis between the tumor and diaphragm motion respiratory motion profiles.

\begin{tabular}{|c|c|c|c|c|c|}
\hline \multirow[b]{3}{*}{ Plan } & \multirow[b]{3}{*}{ Motion } & \multirow{3}{*}{$\begin{array}{c}\text { Irradiation } \\
\quad \#\end{array}$} & \multicolumn{3}{|c|}{ Dose (cGy) } \\
\hline & & & & \multicolumn{2}{|c|}{ Measurements } \\
\hline & & & $\mathrm{MC}$ & Film & RADPOS \\
\hline \multirow{8}{*}{ Static $3 \times 3 \mathrm{~cm}^{2}$} & Sinusoidal & - & $84.4 \pm 0.4 \%$ & $84.1 \pm 2.3 \%$ & $83.8 \pm 2.4 \%$ \\
\hline & $\begin{array}{l}\text { Large } \\
\text { hysteresis }\end{array}$ & - & $72.1 \pm 0.4 \%$ & $71.8 \pm 2.3 \%$ & $73.5 \pm 2.4 \%$ \\
\hline & & 1 & $70.9 \pm 0.4 \%$ & $70.9 \pm 2.3 \%$ & $70.9 \pm 2.4 \%$ \\
\hline & Typical & 2 & $77.3 \pm 0.4 \%$ & $78.7 \pm 2.3 \%$ & $77.8 \pm 2.4 \%$ \\
\hline & & 3 & $79.8 \pm 0.4 \%$ & $79.5 \pm 2.3 \%$ & $79.4 \pm 2.4 \%$ \\
\hline & & 1 & $97.4 \pm 0.4 \%$ & $98.6 \pm 2.3 \%$ & $97.2 \pm 2.4 \%$ \\
\hline & $\begin{array}{l}\text { Highly } \\
\text { irregular }\end{array}$ & 2 & $93.2 \pm 0.4 \%$ & $95.0 \pm 2.3 \%$ & $95.1 \pm 2.4 \%$ \\
\hline & & 3 & $88.6 \pm 0.4 \%$ & $90.4 \pm 2.3 \%$ & $91.1 \pm 2.4 \%$ \\
\hline \multirow{8}{*}{ VMAT } & Sinusoidal & - & $97.1 \pm 0.4 \%$ & $98.7 \pm 2.3 \%$ & $99.0 \pm 2.4 \%$ \\
\hline & $\begin{array}{l}\text { Large } \\
\text { hysteresis }\end{array}$ & - & $98.8 \pm 0.4 \%$ & $98.9 \pm 2.3 \%$ & $98.0 \pm 2.4 \%$ \\
\hline & & 1 & $98.8 \pm 0.4 \%$ & $99.3 \pm 2.3 \%$ & $100.0 \pm 2.4 \%$ \\
\hline & Typical & 2 & $99.4 \pm 0.4 \%$ & $99.6 \pm 2.3 \%$ & $100.5 \pm 2.4 \%$ \\
\hline & & 3 & $97.8 \pm 0.4 \%$ & $97.3 \pm 2.3 \%$ & $100.3 \pm 2.4 \%$ \\
\hline & Highly & 1 & $104.3 \pm 0.4 \%$ & $104.5 \pm 2.3 \%$ & $104.3 \pm 2.4 \%$ \\
\hline & irregular & 2 & $102.0 \pm 0.4 \%$ & $102.6 \pm 2.3 \%$ & $101.2 \pm 2.4 \%$ \\
\hline & & 3 & $101.0 \pm 0.4 \%$ & $103.0 \pm 2.3 \%$ & $100.0 \pm 2.4 \%$ \\
\hline
\end{tabular}


Table 6.12 Dose values at the bottom surface of the plug from MC simulations and RADPOS measurements on the breathing deformable phantom during sinusoidal, typical, highly irregular and large hysteresis between the tumor and diaphragm motion respiratory motion profiles.

\begin{tabular}{|c|c|c|c|c|}
\hline \multirow[b]{3}{*}{ Plan } & \multirow[b]{3}{*}{ Motion } & \multirow{3}{*}{$\begin{array}{c}\text { Irradiation } \\
\#\end{array}$} & \multicolumn{2}{|c|}{ Dose (cGy) } \\
\hline & & & \multicolumn{2}{|c|}{ Bottom surface } \\
\hline & & & $\mathrm{MC}$ & RADPOS \\
\hline \multirow{10}{*}{ Static $3 \times 3 \mathrm{~cm}^{2}$} & Sinusoidal & - & $70.4 \pm 0.4 \%$ & $71.1 \pm 2.4 \%$ \\
\hline & Large & - & $551+04 \%$ & $525+210 \%$ \\
\hline & hysteresis & & & \\
\hline & & 1 & $51.9 \pm 0.4 \%$ & $51.6 \pm 2.4 \%$ \\
\hline & Typical & 2 & $56.6 \pm 0.4 \%$ & $55.0 \pm 2.4 \%$ \\
\hline & & 3 & $58.2 \pm 0.4 \%$ & $58.3 \pm 2.4 \%$ \\
\hline & & 1 & $77.2 \pm 0.4 \%$ & $77.9 \pm 2.4 \%$ \\
\hline & Highly & 2 & $753+04 \%$ & $773+240$ \\
\hline & irregular & & $1 J .5+0.7 / 0$ & $17.0 \pm 2.78$ \\
\hline & & 3 & $71.3 \pm 0.4 \%$ & $73.6 \pm 2.4 \%$ \\
\hline \multirow{10}{*}{ VMAT } & Sinusoidal & - & $73.8 \pm 0.4 \%$ & $73.5 \pm 2.4 \%$ \\
\hline & Large & & & \\
\hline & hysteresis & - & $65.7 \pm 0.4 \%$ & $63.5 \pm 2.4 \%$ \\
\hline & & 1 & $66.0 \pm 0.4 \%$ & $65.8 \pm 2.4 \%$ \\
\hline & Typical & 2 & $65.5 \pm 0.4 \%$ & $66.8 \pm 2.4 \%$ \\
\hline & & 3 & $65.8 \pm 0.4 \%$ & $63.3 \pm 2.4 \%$ \\
\hline & & 1 & $82.0 \pm 0.4 \%$ & $80.6 \pm 2.4 \%$ \\
\hline & Highly & & & \\
\hline & irregular & 2 & $79.0 \pm 0.4 \%$ & $78.0 \pm 2.4 \%$ \\
\hline & & 3 & $77.4 \pm 0.4 \%$ & $76.2 \pm 2.4 \%$ \\
\hline
\end{tabular}


Table 6.13 Dose values at the top surface of the plug from MC simulations and RADPOS measurements on the breathing deformable phantom during sinusoidal, typical, highly irregular and large hysteresis between the tumor and diaphragm motion respiratory motion profiles. This RADPOS was placed at an offset position of $1 \mathrm{~cm}$ from the tumor center.

\begin{tabular}{|c|c|c|c|c|}
\hline \multirow[b]{3}{*}{ Plan } & \multirow[b]{3}{*}{ Motion } & \multirow{3}{*}{$\begin{array}{c}\text { Irradiation } \\
\#\end{array}$} & \multicolumn{2}{|c|}{ Dose (cGy) } \\
\hline & & & \multicolumn{2}{|c|}{ Top surface } \\
\hline & & & $\mathrm{MC}$ & RADPOS \\
\hline \multirow{8}{*}{ Static $3 \times 3 \mathrm{~cm}^{2}$} & Sinusoidal & - & $38.1 \pm 0.4 \%$ & $37.2 \pm 2.2 \%$ \\
\hline & $\begin{array}{l}\text { Large } \\
\text { hysteresis }\end{array}$ & - & $18.0 \pm 0.4 \%$ & $18.5 \pm 2.2 \%$ \\
\hline & & 1 & $13.8 \pm 0.4 \%$ & $13.2 \pm 2.2 \%$ \\
\hline & Typical & 2 & $15.4 \pm 0.4 \%$ & $15.0 \pm 2.2 \%$ \\
\hline & & 3 & $16.4 \pm 0.4 \%$ & $15.9 \pm 2.2 \%$ \\
\hline & & 1 & $17.1 \pm 0.4 \%$ & $17.9 \pm 2.2 \%$ \\
\hline & $\begin{array}{l}\text { Highly } \\
\text { irregular }\end{array}$ & 2 & $17.4 \pm 0.4 \%$ & $18.2 \pm 2.2 \%$ \\
\hline & & 3 & $14.8 \pm 0.4 \%$ & $15.1 \pm 2.2 \%$ \\
\hline \multirow{8}{*}{ VMAT } & Sinusoidal & - & $41.0 \pm 0.4 \%$ & $39.9 \pm 2.2 \%$ \\
\hline & $\begin{array}{l}\text { Large } \\
\text { hysteresis }\end{array}$ & - & $22.9 \pm 0.4 \%$ & $23.6 \pm 2.2 \%$ \\
\hline & & 1 & $21.8 \pm 0.4 \%$ & $22.6 \pm 2.2 \%$ \\
\hline & Typical & 2 & $23.8 \pm 0.4 \%$ & $24.0 \pm 2.2 \%$ \\
\hline & & 3 & $22.3 \pm 0.4 \%$ & $23.4 \pm 2.2 \%$ \\
\hline & Highly & 1 & $33.2 \pm 0.4 \%$ & $34.1 \pm 2.2 \%$ \\
\hline & irregular & 2 & $31.8 \pm 0.4 \%$ & $32.5 \pm 2.2 \%$ \\
\hline & & 3 & $32.9 \pm 0.4 \%$ & $33.8 \pm 2.2 \%$ \\
\hline
\end{tabular}


From the values in Table 6.11 we can see that for the majority of the irradiations, the measured and simulated dose values at the center of tumor agree within $2 \%$ of each other. Exceptions are the third beam deliveries of the static $3 \times 3 \mathrm{~cm}^{2}$ plan for the highly irregular motion and the VMAT plan for the typical respiratory motion that show agreements of better than 3\% between MC simulations and RADPOS measurements which still lies within the $2 \sigma$ of the experimental uncertainties (4.8\%). Overall, for the irradiations done for the respiratory motions with sinusoidal and large hysteresis between the tumor and diaphragm motion traces, the same level of agreement (i.e. better than $3 \%$ ) was found to be true. In addition, from dose profiles shown in Figures 6.16-6.19, we can see that the dose gradient can increase due to respiratory motion. This is especially prominent for the $3 \times 3 \mathrm{~cm}^{2}$ plan which adds an intrinsic dose gradient compared to the VMAT plan. For some motion traces (typical and large hysteresis between the tumor and diaphragm motion), total positional/reading uncertainties of dose values can be as high as $8 \%$ for the $3 \times 3 \mathrm{~cm}^{2}$ plan which is observable from the level of dose gradient in profiles in Figures 6.17 and 6.19. Considering that the largest component of the motion happens in the S-I direction, such uncertainties could be twice as the overall values if we take only S-I direction into consideration. For other motion traces on the other hand, these uncertainties did not exceed $3-4 \%$.

At the bottom surface of the plug, measured and simulated dose values shown in Table 6.12 show a better than $3 \%$ level of agreement. Similar to the tumor dose values, exceptions are the two same irradiations which do not show a disagreement larger than $4 \%$ which is within the $2 \sigma$ of the experimental uncertainties $(4.8 \%)$ as well. The total level of 
agreement for all irradiations for the respiratory motions with sinusoidal and large hysteresis between the tumor and diaphragm motion traces were observed to be within $4 \%$. Once studied, approximately the same level of dose gradient and as a result dose reading/positional uncertainty as for the center of tumor was observed at this dose point.

As for dose values at the top surface of the plug that are shown in Table 6.13, an agreement of better than $5 \%$ is observable which complies with the fact that this dose point is initially positioned in a high dose gradient region. It should be noted that since this point dose is placed close to the edge of the beam, the motion results in a larger drop in the dose value compared to dose values at the tumor center and the bottom surface. The overall dose reading/positional uncertainties are approximately $12 \%$ and $10 \%$ for the $3 \times 3 \mathrm{~cm}^{2}$ and VMAT plan deliveries, while considering only the S-I direction these values can go as high as $20 \%$. In average, same level of dose difference were observed for all irradiations with the sinusoidal and large hysteresis between the tumor and diaphragm motion respiratory motion profiles with some dose differences slightly larger than $5 \%$.

In order to explain the dose differences observed between three irradiations (beamon at respectively 0, 40 and $90 \mathrm{~s}$ after the motion starts) for the typical and highly irregular motion profiles, respiratory traces recorded with RADPOS during beam deliveries were extracted. The fraction of time that was spent in each respiratory phase is shown in Figures 6.20 and 6.21 for these two traces, respectively, for both plan deliveries. 

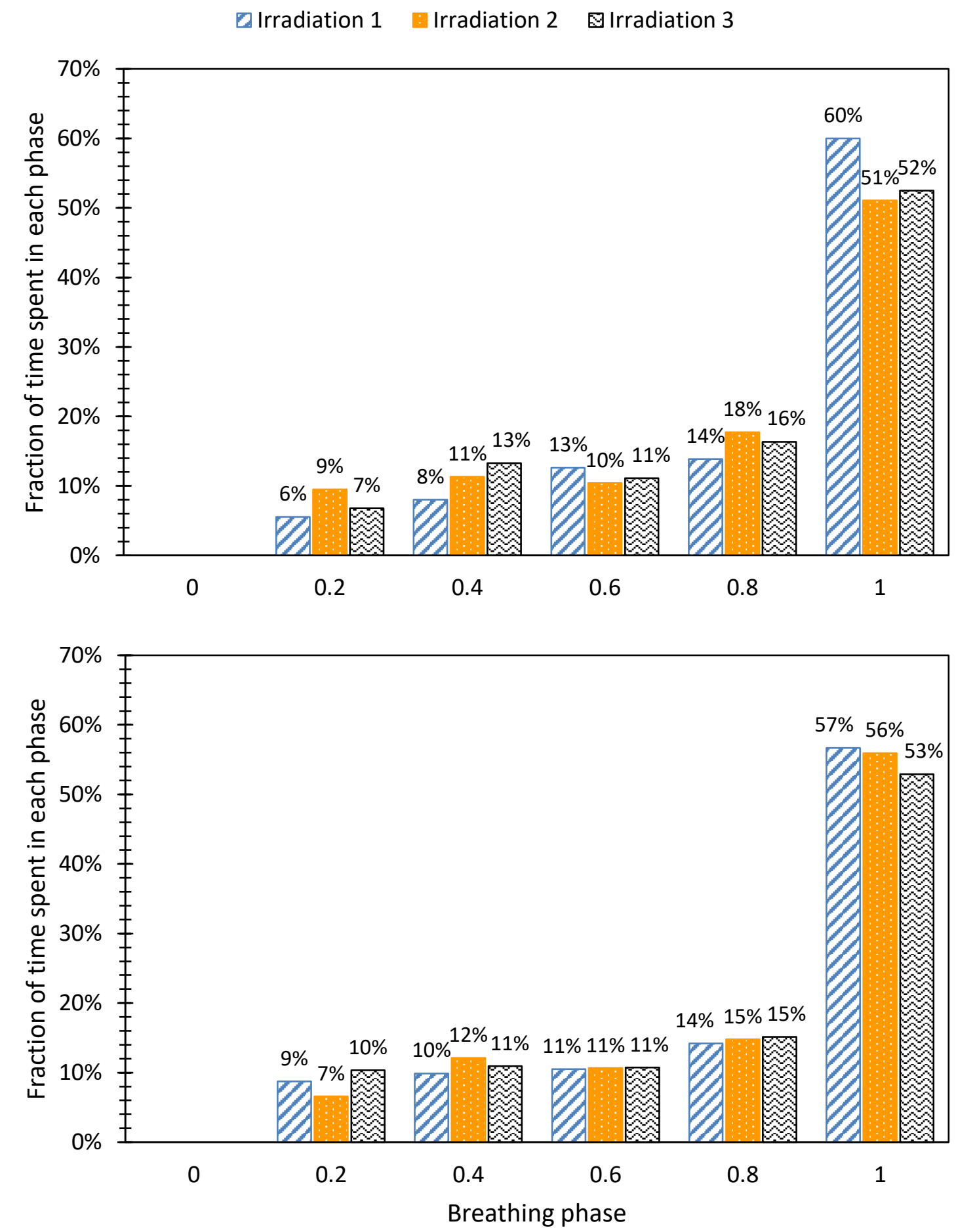

Figure 6.21 Fraction of the time spent in each breathing phase during $3 \times 3 \mathrm{~cm}^{2}$ (top) and VMAT (bottom) beam deliveries on the deforming phantom for three irradiations with the typical 
respiratory motion profile shown in Figure 5.9 (a). Breathing phase is the RADPOS normalized displacement that was recorded during beam deliveries.
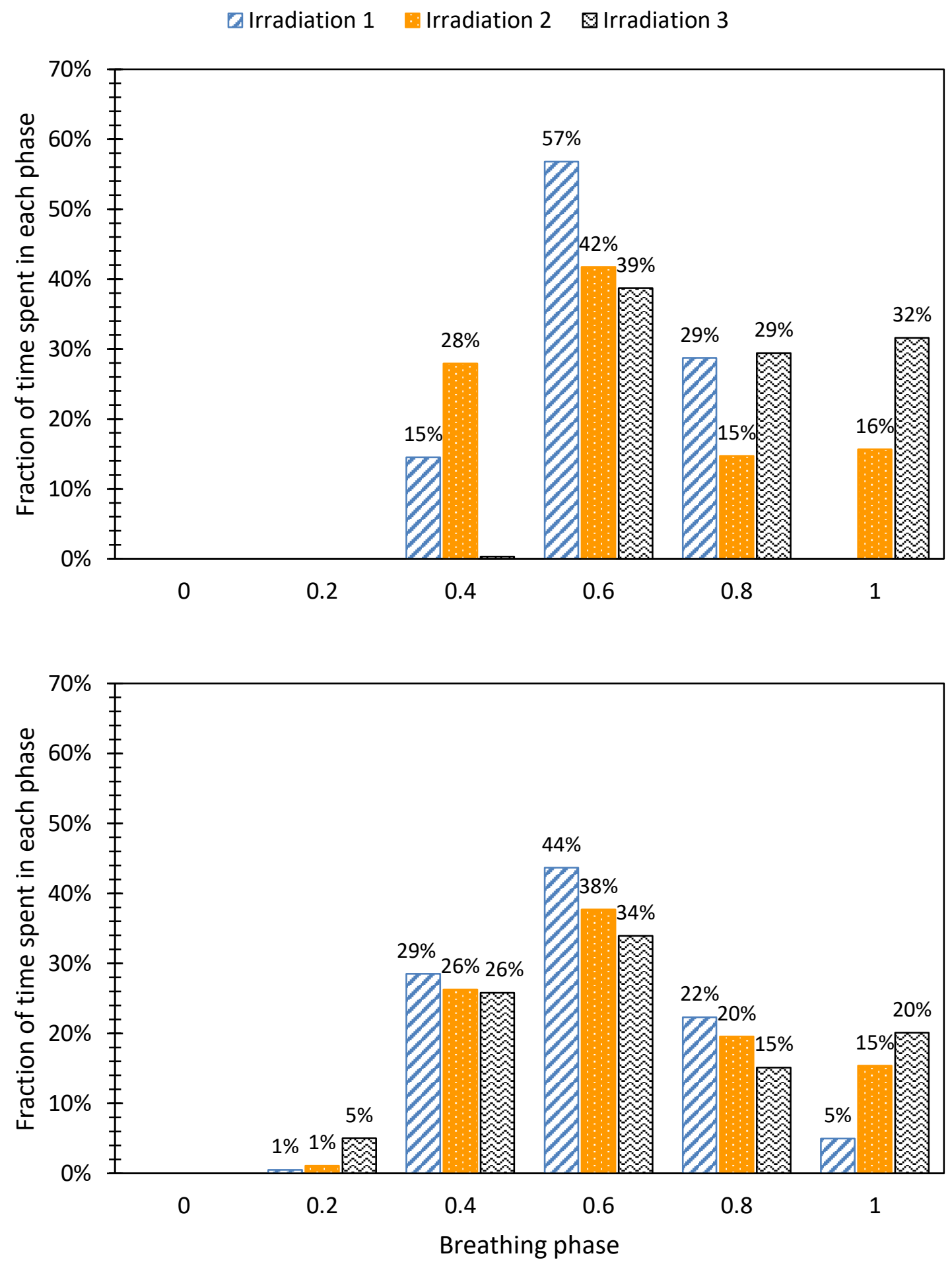

Figure 6.22 Fraction of the time spent in each breathing phase during $3 \times 3 \mathrm{~cm}^{2}$ (top) and VMAT (bottom) beam deliveries on the deforming phantom for three irradiations with the highly 
irregular respiratory motion profile shown in Figure 5.9 (b). Breathing phase is the RADPOS normalized displacement that was recorded during beam deliveries.

From the values shown in Figure 6.20 we can see that $60 \%$ of the first static $3 \times 3 \mathrm{~cm}^{2}$ beam delivery happened while the phantom was in the EOE (fully compressed or breathing phase 1) state, whereas for the other two beam deliveries this value reduces to approximately $50 \%$. When studied in more detail, it was observed that $45 \%$ of the total time spent in EOE for the first irradiation was spent in this state without any transitions to EOI (non-compressed, breathing phase 0). This explains the lower dose values in the first irradiation compared to the two other irradiations. As for VMAT deliveries, it can be seen that almost equal times are spent in each phase for the three irradiations since VMAT plans take longer time compared to static plans ( $\sim 67 \mathrm{~s}$ vs $\sim 15 \mathrm{~s})$ to deliver.

For the static $3 \times 3 \mathrm{~cm}^{2}$ beam delivery of the highly irregular motion profile, it can be seen in the top plot of Figure 6.21 that over $70 \%$ of the beam delivery was done while the phantom was in the early- and mid-inhale states. However, looking at the last irradiation, only $40 \%$ of the beam was delivered in this state and the remaining $60 \%$ was delivered when the phantom was in the mid-exhale and EOE states. As a result, the dose delivered during this beam delivery was lower compared to the first two deliveries. On the second plot related to the VMAT delivery it can be seen that approximately $75 \%$ of the first irradiation happened while the phantom was in the early- and mid-inhale states which is almost $10-15 \%$ higher than similar values for the two last irradiations. As a result, we can see some dose differences between the deliveries in this case. 


\subsection{Discussion}

In this study 4D Monte Carlo simulations using the 4DdefDOSXYZnrc user code to calculate the dose delivered to rigidly moving (Quasar phantom) as well as deforming (deformable phantom) anatomies were validated. Static and VMAT plans were delivered to the phantoms in stationary and breathing states. The Quasar phantom was moved with sinusoidal breathing motion. Sinusoidal and irregular breathing motions were used on the programmable deformable phantom to simulate more realistic respiratory motion. Film was placed inside the lung inserts of the phantoms to measure dose distributions along their motion paths (S-I). Point dose measurements with RADPOS were performed inside the GTV (i.e. tumor) for both phantoms. For the deformable phantom, point doses were also measured outside the tumor (still inside the lung). Recorded displacements with RADPOS inside the GTV as well as manually generated displacement vectors (Quasar phantom) or DVFs generated by DIR (deformable phantom) were used as input to 4DMC simulations to model the motions/deformations of phantoms.

Our results showed that the overall agreement of point dose values at the center of the tumor from $\mathrm{MC}$ simulations and measurements by film were within $2 \%$ of each other. Dose differences between RADPOS measurements and MC simulations did not exceed 3\% which is not larger than $2 \sigma$ of the experimental uncertainties with RADPOS measurements. As for the dose points outside the tumor (i.e. top and bottom surfaces of the plug) in the deformable phantom, simulations and measurements were found to have an average agreement of $6 \%$ or better. These agreements were found to be within the calculated positional uncertainties of these dose points. The agreements between the simulated and measured dose profiles (along the S-I direction) as well as 2D dose distributions (Quasar 
phantom), were good as well. Gamma comparisons of $2 \% / 2 \mathrm{~mm}$ showed an overall passing rate of almost $94 \%$ or better.

In order to investigate the impact of starting phase of the respiratory cycle, treatments for both plans were started at 0,40 and $90 \mathrm{~s}$ after the deformable phantom started to move with typical and highly irregular respiratory profiles. Different doses can be measured depending on the amount of time a target volume spends in EOI, EOE or transitions between these two states. In this work it was observed that for static plan deliveries, the dose delivered to the tumor as well as the bottom surface of the plug could change by $10-12 \%$ once they spent almost $45 \%$ of the delivery time in the EOE without any transitions to EOI. Similar fractions of time spent in EOE resulted in a dose change of $15-20 \%$ at the dose point on the top surface of the tumor due to the fact that it was in the penumbra region of the beam. For VMAT deliveries, the longer treatment times compared to static deliveries help even out the impact of differences in the respiratory cycle during several deliveries of the same treatment plan. As a result, dose differences between deliveries may not be as large as seen for static treatment plans. In the case of VMAT deliveries for the typical breathing trace where the target volume spends almost equal amounts of time in the EOE and EOI during different deliveries, dose differences of less than $1 \%$ could be observed. On the other hand, with highly irregular respiratory traces the case could be different. Differences of almost 3-4\% were observed for this respiratory profile between the one VMAT beam delivery with $10-15 \%$ more time spent in early-, midinhale compared to the other two deliveries.

Comparison of dose values from the static beam deliveries for the respiratory motions with sinusoidal and large hysteresis between the tumor and diaphragm motion 
traces revealed approximate dose decreases of $15 \%, 20 \%$ and $50 \%$ at the center of tumor, bottom and top surfaces of the plug, respectively. This result was expected considering the time spent in each breathing phase during beam deliveries for each of these motion profiles. While moving the sinusoidal motion, these dose points spend almost equal amounts of times from EOI-EOE and EOE-EOI. However, these times change to $20 \%$ and $80 \%$ while the phantom moves with the respiratory motion trace with the large hysteresis between the tumor and diaphragm motion.

The difference in temporal resolutions of RADPOS (100 ms) and log files (40 ms) could be one potential source of experimental uncertainty, as it impacts the accuracy of the detecting starting phase of respiratory cycle. Depending on the frequency and amplitude of motions used in this work, these system delays can convert to positional inaccuracies of up to $1 \mathrm{~mm}$ in the S-I direction. Moreover, the PC clock synchronization between linac and RADPOS computers could be another source of uncertainty to determine the starting phase of motion. In addition, we have the position measurement stability of RADPOS which is better than $0.2 \mathrm{~mm}$. After investigation, it was observed that such positional uncertainties can cause point dose deviations of approximately $1-2 \%$ in the target volume as well as bottom surface of the plug. Dose value at the top surface of the plug is more sensitive to such positional uncertainties as a result of being placed in high dose gradient region. Thus, dose deviations of as large as 3-4\% might be observed at this point. Accurate detection of the starting phase of the respiratory cycles becomes more essential when delivering highly modulated plans (due to the interplay effect) or plan with shorter delivery times.

One important aspect of 4D simulations is the use of displacement vectors or DVFs. For a rigidly translating anatomy like the lung insert of the Quasar phantom, the use of DIR 
algorithms to generate displacement vectors is not an appropriate choice. However, the use of DIR to generate DVFs is necessary and a common practice to model deformations occurring in a deforming anatomy such as the deformable phantom used in this work. This means that the accuracy of simulations will depend on the accuracy of the DIR algorithm as well. Thus, the challenge here will be to generate DVFs that are as accurate as possible. The DVFs used in our work were found to have accuracy better than $2 \mathrm{~mm}$ in 3D trajectories. The impact of larger/smaller motion amplitudes on dose values for a sinusoidal breathing trace were investigated by introducing error values to the DVFs. Since the largest motion corresponds to SI direction, change in the dose due to change in this component was studied. The amplitude was changed by $4 \%$ (higher and lower) which converted to about \pm 0.6 and $\pm 0.5 \mathrm{~mm}$ at the tumor center and top and bottom plug surfaces, respectively. These values compensate approximately for the part of the variations between motion amplitudes measured with RADPOS and modeled by DVFs. The impact on static and VMAT plans and for each dose measurement point was studied separately. For the static plan, decrease of approximately $4 \%$ in dose can happen at the center of tumor when larger amplitudes were used. The reason for such deviations is the fact that this point dose leaves the radiation field once the phantom enters the EOE state. For smaller amplitude of motion dose was increased only by $1-2 \%$ since dose point still remains in the radiation field. Looking into similar values for the VMAT plan, it was observed that dose increased/decreased by $1-2 \%$. As these calculated values suggest, dose deviations for the VMAT plan are not as large as for the static plan. The main reason is the wider radiation field and as a result, lower dose gradient of the VMAT compared to the static plan. For both plan deliveries, at the bottom surface of the plug, dose was deviated by $1-2 \%$ due to 
the change in the motion amplitude. Dose deviations at the point dose at top surface of the plug, were observed to be over $4 \%$ for both plans because it was originally in a high dose gradient region. Like positional uncertainties, DVF errors and differences in the simulated vs. measured amplitudes are other sources of uncertainty that can account for differences observed between calculated and measured dose values.

A further possible source of uncertainty is the interpolation between the control points as performed by source 21 in MC simulations. However, these control points are defined according to delivery log files with a high sampling rate of $40 \mathrm{~ms}$. Analyzing delivery log files revealed that this sampling rate corresponds to average change of $0.2^{\circ}$ and $0.1 \mathrm{~mm}$ in the gantry angle and MLC positions between two consecutive interpolation points, respectively. With respect to MU fraction, this change does not exceed 0.001 . Thus, high sampling rate of $\log$ files help minimizing the potential errors caused by interpolations between control points.

\subsection{Conclusions}

We investigated and established the accuracy of 4D Monte Carlo simulations, using the 4DdefDOSXYZnrc user code, of dose delivered to the rigidly moving and deforming phantoms during sinusoidal breathing motion profiles. Measurements were performed on an Elekta Infinity linac equipped with Agility MLC during static square and VMAT plan deliveries. Delivery log files were used to reproduce the measurements during these deliveries. Also, we validated our method by similar dose deliveries in the presence of more realistic motion profiles with a programmable deformable phantom. Our findings demonstrate that combining position tracing with RADPOS and DVFs generated by a reliable DIR algorithm (to model motion and deformation of the anatomy) with our 4DMC 
simulations, leads to accurate calculation of cumulative dose delivered to patients which can be used for adaptive purposes. 


\section{Chapter 7}

\section{Summary and Outlook}

The aim of this study was to experimentally validate, for the first time, a novel 4D Monte Carlo (MC) tool which calculates the dose distribution delivered to a continuously moving/deforming anatomy and includes continuous motion of the linac components for static and VMAT treatment techniques.

Chapter 4 presents our Monte Carlo modelling of the full treatment head for the Elekta Infinity linac equipped with Agility MLC that was used for this work. The model was initially created in BEAMnrc and then model parameters were tuned by comparing MC calculated and measured dosimetric parameters like PDD curves, RDFs, etc. We present a unique approach to determine the correct LBROT value that was determined to be 9 mrad. Our method includes comparing MC simulations with different LBROT values against film measurements for alternating open-closed collimator leaves. It was possible to use the same MC model to study the sensitivity of model parameters (e.g. source size, LBROT). However, a simpler and faster method was to use a virtual source model of the linac treatment head, including primary and secondary photon sources. This model was adopted to analytically calculate the photon fluence at the isocenter plane from a small radiation field. This virtual source model also proved to be a valuable tool to help explain dose discrepancies observed between film measurements/BEAMnrc calculations and Monaco TPS calculations for a small field due to an alternating open-closed pattern. These dose deviations were determined to be approximately $15 \%$ and $40 \%$ at the position of maxima and minima. Finally, as it was shown, MC model parameters (source size and 
inclusion of tongue \& groove in the MLC) were modified to match Monaco which improved the agreements at the position of maxima and minima by almost $10 \%$ and $30 \%$, respectively, resulting in agreements of better than $2 \%$.

Chapter 5 introduced the respiratory lung phantoms that were used for experimental work in this study. A novel programmable deformable lung phantom was designed and characterized. This phantom simulates tissue characteristics of lung and includes an elastic tumor. The phantom was designed to include film and RADPOS 4D dosimetry system probe to allow both dose and position verification. Since it is important to simulate realistic breathing traces for lung studies, the phantom was designed to be programmed with arbitrary respiratory motion profiles. In addition to lung tissue-equivalency, the phantom motion showed levels of hysteresis throughout several locations. Once tested for sinusoidal and irregular respiratory patterns, the phantom showed a motion reproducibility of about 1 $\mathrm{mm}$.

Experimental verification of the 4DMC simulations using the 4DdefDOSXYZnrc user code are covered in Chapter 6. The 4DdefDOSXYZnrc code calculates the dose deposition by incident radiation in a continuously deforming voxelized geometry. The magnitude of displacements/deformations are determined by displacement vector fields (DVFs) generated through a registration process. One challenge is to ensure the highest possible accuracy of DVFs since they have a direct impact on the accuracy of MC simulations. In this work, a 3D accuracy of better than $2 \mathrm{~mm}$ was achieved. The RADPOS 4D dosimetry system was used during treatments to record the respiratory motion trace with a temporal resolution of $100 \mathrm{~ms}$. This motion trace was then combined with the DVFs to model the motion and deformation of the lung. Once combined with delivery log files, 
we implemented both anatomy and machine motion (using source 21 of DOSXYZnrc) into our 4DMC simulations. Attention needs to be paid during measurements to ensure synchronization between the beam-on time and starting phase of the breathing cycle to reduce calculation errors caused by positional uncertainties. Both point dose and dose profiles were acquired during measurements and were compared against $\mathrm{MC}$ simulations. Overall and for all respiratory traces that were tested in this work, point dose agreements between MC simulations and measurements (film and RADPOS) were within $2 \sigma$ of experimental and/or positional/dose reading uncertainties. The agreements between dose profiles from film measurements and MC simulations were determined to be within $2 \% / 2$ $\mathrm{mm}$. By studying the impact of different breathing phases of the same respiratory pattern, we found that the amount of time spent in different breathing phases has a larger impact on the dose delivered to a target volume during static treatments compared to VMAT treatments.

This work demonstrates the potential of 4DMC simulations using 4DdefDOSXYZnrc user code to be used as a quality assurance tool to verify lung treatments in combination with RADPOS, delivery log files and realistic deformable phantoms. One main drawback of these simulations is that they require long CPU-hours to complete. Improvement to the user code to achieve much faster and more efficient dose calculations is a requirement before the code is ready for clinical implementation.

Moreover, we demonstrated that respiratory traces recorded by RADPOS can be used to correlate the motion of different points of an anatomy (e.g. internal-external motion). Thus, RADPOS mounted outside an anatomy can be used as a surrogate to create patient-specific respiratory motion models of the internal anatomy and calculate the dose 
delivered to a target volume in the patient. More experimental work with the deformable phantom is required to ensure the accuracy of 4D simulations using such respiratory motion models before clinical verifications with real patients. Modifications to the phantom might be required to allow mounting RADPOS on the external surface of the phantom as well as internal parts.

One improvement to the design of the phantom could be modifying it to allow insertion of the plug holding the tumor and dosimeters in different A-P, L-R and S-I locations rather than just center of the phantom. This modification would enable studying the impact of the tumor location (e.g. different lobes) on the change in the motion/deformations inside the tumor as well as the dose delivered to it. One consideration with this change is insertion of the film if the tumor is placed close to the diaphragm due to the higher level of motion/deformation in the S-I direction. Also, despite its novel and realistic design, our phantom has limitations in simulating all observed motion types that occur in the chest. For example, the phantom with its current design is not able to simulate the sliding motion between different lung lobes.

This study needs to be extended to more realistic situations that happen in the clinic. The patient's respiratory patterns (amplitudes and periods) could be different during each treatment fraction from the breathing pattern recorded during the acquisition of 4DCT images. These differences could introduce errors in the calculated dose since 4D simulations use the DVFs generated through deformable registration of inhale and exhale CT images to extract the range of motion. More work is required to investigate these issues. One needs to explore if there is a need for modification (e.g. extrapolation) of the 
deformation vectors to match recorded traces during each treatment fraction, in order to avoid calculation errors.

The linear interpolation of the DVFs between inhale and exhale breathing phases used in these simulations could be a potential source of uncertainty if the temporal resolution of the respiratory motion trace is low. Such uncertainties would especially become significant in the presence of irregular traces or large hysteresis between the tumor and diaphragm motion effects of the phantom. Thus, it is important to ensure acquiring motion traces with high sampling rates and investigate the potential errors introduced otherwise, before clinical implementations. Currently two extreme respiratory phases (i.e. EOE and EOI) are used to generate DVFs required for motion model. More work is required to investigate the need to use more respiratory phases.

Although the results of this work are extendable to measurements/simulations with other conventional linacs (e.g. Varian) or radiotherapy devices (e.g. Helical TomoTherapy and CyberKnife), it is important to validate simulations with those units due to their different specifications. In addition, more work is still required to verify these simulations in the presence of more complicated treatment techniques (e.g. inclusion of couch rotation or multiple beams).

RADPOS can safely be attached to the surface of patients (e.g. diaphragm or chest) without causing any discomfort to record position and to measure dose during treatment deliveries to be verified against equivalent 4DMC simulations. Thus, clinical trials can be run before this tool can be widely used in the clinic. Based on results of such trials, adaptive treatment delivery is another area that can benefit from the results of this work. 


\section{References}

1 Canadian Cancer Society Advisory Committee on Cancer Statistics, A 2018 special report on cancer incidence by stage, Canadian Cancer Society (2018).

${ }^{2}$ G. Delaney, S. Jacob, C. Featherstone, and M. Barton, The role of radiotherapy in cancer treatment: Estimating optimal utilization from a review of evidence-based clinical guidelines, Cancer 104(6), 1129-1137 (2005).

${ }^{3}$ E. B. Podgorsak, Radiation Oncology Physics: A Handbook for Teachers and Students, IAEA Educational Report series (2008).

${ }^{4}$ C. Ozhasoglu, C.B. Saw, H. Chen, et al., Synchrony-cyberknife respiratory compensation technology, Med. Dosim. 33(2), 117-123 (2008).

${ }^{5}$ S. Vahdat, E.K. Oermann, S.P. Collins, et al., CyberKnife radiosurgery for inoperable stage IA non-small cell lung cancer: 18F-fluorodeoxyglucose positron emission tomography/computed tomography serial tumor response assessment, J. Hematol. Oncol. 3(1), 6 (2010).

${ }^{6}$ C. Ding, C. Chang, J. Haslam, R. Timmerman, and T. Solberg, A dosimetric comparison of stereotactic body radiation therapy techniques for lung cancer: robotic versus conventional linac-based systems, J. Appl. Clin. Med. Phys. 11(3), 212-224 (2010).

${ }^{7}$ B.T. Collins, S. Vahdat, K. Erickson, et al., Radical cyberknife radiosurgery with tumor tracking: an effective treatment for inoperable small peripheral stage I non-small cell lung cancer, J. Hematol. Oncol. 2(1), 1 (2009). 
${ }^{8}$ H. Bahig, M.-P. Campeau, T. Vu, et al., Predictive parameters of CyberKnife fiducialless (XSight Lung) applicability for treatment of early non-small cell lung cancer: a single-center experience, Int. J. Radiat. Oncol. Biol. Phys. 87(3), 583-589 (2013).

${ }^{9}$ AAPM Report No. 46, Comprehensive QA for radiation oncology: Report of Task Group 40 of the Radiation Therapy Committee (1994).

${ }^{10}$ B. Fraass, K. Doppke, M. Hunt, G. Kutcher, G. Starkschall, R. Stern, and J. Van Dyke, Quality assurance for clicnial radiotherapy treatment planning, Report of AAPM Task Group 53., Med. Phys. 25(10), 1773-1829 (1995).

${ }^{11}$ International Commission on Radiation Units and Measurements, ICRU Report 50: Prescribing, recording, and reporting photon beam therapy, ICRU (1993).

12 ICRU Report 62: Prescribing, recording, and reporting photon beam therapy (Supplement to ICRU Report 50), ICRU (1999).

${ }^{13}$ A. Boyer, B. Butler, T. DiPetrillo, et al., Intensity-Modulated Radiotherapy: Current status and issues of interest, Intensity Modulated Radiation Therapy Collaborative Working Group, Int. J. Radiat. Oncol. Biol. Phys. 51(4), 880-914 (2001).

14 K. Otto, Volumetric modulated arc therapy: IMRT in a single gantry arc, Med. Phys. 35(1), 310-317 (2008).

15 T.A. Sundaram and J.C. Gee, Towards a model of lung biomechanics: pulmonary kinematics via registration of serial lung images, Med. Image Anal. 9(6), 524-537 (2005).

16 S. Ukil and J.M. Reinhardt, Anatomy-guided lung lobe segmentation in X-ray CT 
images, IEEE Trans. Med. Imaging 28(2), 202-214 (2009).

17 A. Ratnovsky, D. Elad, and P. Halpern, Mechanics of respiratory muscles, Respir. Physiol. Neurobiol. 163(1-3), 82-89 (2008).

${ }^{18}$ G. Zhang, X. Chen, J. Ohgi, et al., Biomechanical simulation of thorax deformation using finite element approach, Biomed. Eng. Online 15(1), 18 (2016).

19 J.R. McClelland, D.J. Hawkes, T. Schaeffter, and A.P. King, Respiratory motion models: a review, Med. Image Anal. 17(1), 19-42 (2013).

${ }^{20}$ T. Bortfeld, S. Jiang, and E. Rietzel, Effects of motion on the total dose distribution, Semin. Radiat. Oncol. 14(1), 41-51 (2004).

${ }^{21}$ P.J. Keall, G.S. Mageras, J.M. Balter, et al., The management of respiratory motion in radiation oncology, Report of AAPM Task Group 76, Med. Phys. 33(10), 3874-3900 (2006).

${ }^{22}$ K.M. Langen and D.T.L. Jones, Organ motion and its management, Int. J. Radiat. Oncol. Biol. Phys. 50(1), 265-278 (2001).

${ }^{23}$ M. Palombarini, S. Mengoli, P. Fantazzini, C. Cadioli, C. Degli Esposti, and G.P. Frezza, Analysis of inter-fraction setup errors and organ motion by daily kilovoltage cone beam computed tomography in intensity modulated radiotherapy of prostate cancer, Radiat. Oncol. 7(56), 1-8 (2012).

${ }^{24}$ J.D. Lawson, T. Fox, E. Elder, et al., Early clinical experience with kilovoltage imageguided radiation therapy for interfraction motion management, Med. Dosim. 33(4), $268-274$ (2008). 
25 S. Flampouri, S.B. Jiang, G.C. Sharp, J. Wolfgang, A.A. Patel, and N.C. Choi, Estimation of the delivered patient dose in lung IMRT treatment based on deformable registration of 4D-CT data and Monte Carlo simulations, Phys. Med. Biol. 51(11), 2763-79 (2006).

26 H.H. Liu, P. Balter, T. Tutt, et al., Assessing respiration-induced tumor motion and internal target volume using four-dimensional computed tomography for radiotherapy of lung cancer, Int. J. Radiat. Oncol. Biol. Phys. 68(2), 531-540 (2007).

27 S. Sarudis, A. Karlsson Hauer, J. Nyman, and A. Bäck, Systematic evaluation of lung tumor motion using four-dimensional computed tomography, Acta Oncol. 56(4), 525530 (2017).

${ }^{28}$ M. Engelsman, E.M.F. Damen, K. De Jaeger, K.M. Van Ingen, and B.J. Mijnheer, The effect of breathing and set-up errors on the cumulative dose to a lung tumor, Radiother. Oncol. 60(1), 95-105 (2001).

29 S.B. Jiang, C. Pope, K.M. Al Jarrah, J.H. Kung, T. Bortfeld, and G.T.Y. Chen, An experimental investigation on intra-fractional organ motion effects in lung IMRT treatments, Phys. Med. Biol. 48(12), 1773-1784 (2003).

${ }^{30}$ M. Rao, J. Wu, D. Cao, et al., Dosimetric impact of breathing motion in lung stereotactic body radiotherapy treatment using image-modulated radiotherapy and volumetric modulated arc therapy, Int. J. Radiat. Oncol. Biol. Phys. 83(2), e251-e256 (2012).

31 C. Ong, W.F. A. R. Verbakel, J.P. Cuijpers, B.J. Slotman, and S. Senan, Dosimetric 
impact of interplay effect on RapidArc lung stereotactic treatment delivery, Int. J. Radiat. Oncol. 79(1), 305-311 (2011).

${ }^{32}$ L. Court, M. Wagar, R. Berbeco, et al., Evaluation of the interplay effect when using RapidArc to treat targets moving in the craniocaudal or right-left direction, Med. Phys. 37(1), 4-11 (2010)..

33 J. Duan, S. Shen, J.B. Fiveash, R. A Popple, and I. A Brezovich, Dosimetric and radiobiological impact of dose fractionation on respiratory motion induced IMRT delivery errors: a volumetric dose measurement study, Med. Phys. 33 (5), 1380-1387 (2006).

${ }^{34}$ R.I. Berbeco, C.J. Pope, and S.B. Jiang, Measurement of the interplay effect in lung IMRT treatment using EDR2 films, J. Appl. Clin. Med. Phys. 7(4), 33-42 (2006).

${ }^{35}$ C.X. Yu, D. A Jaffray, and J.W. Wong, The effects of intra-fraction organ motion on the delivery of dynamic intensity modulation, Phys. Med. Biol. 43(1), 91-104 (1998).

${ }^{36}$ T. Bortfeld, K. Jokivarsi, M. Goitein, J. Kung, and S.B. Jiang, Effects of intra-fraction motion on IMRT dose delivery: statistical analysis and simulation, Phys. Med. Biol. 47(13), 2203-2220 (2002).

37 J. Hanley, M.M. Debois, D. Mah, et al., Deep inspiration breath-hold technique for lung tumors: The potential value of target immobilization and reduced lung density in dose escalation, Int. J. Radiat. Oncol. Biol. Phys. 45(3), 603-611 (1999).

${ }^{38}$ K.E. Rosenzweig, J. Hanley, D. Mah, et al., The deep inspiration breath-hold technique in the treatment of inoperable non-small-cell lung cancer, Int. J. Radiat. 
Oncol. Biol. Phys. 48(1), 81-87 (2000).

39 K. Nakamura, Y. Shioyama, S. Nomoto, et al., reproducibility of the abdominal and chest wall position by voluntary breath-hold technique using a laser-based monitoring and visual feedback system, Int. J. Radiat. Oncol. Biol. Phys. 68(1), 267-272 (2007).

40 G.S. Mageras and E. Yorke, Deep inspiration breath hold and respiratory gating strategies for reducing organ motion in radiation treatment, Semin. Radiat. Oncol. 14(1), 65-75 (2004).

41 J.W. Wong, M.B. Sharpe, D.A. Jaffray, et al., The use of active breathing control (ABC) to reduce margin for breathing motion, Int. J. Radiat. Oncol. Biol. Phys. 44(4), 911-919 (1999).

42 V.M. Remouchamps, N. Letts, F.A. Vicini, et al., Initial clinical experience with moderate deep-inspiration breath hold using an active breathing control device in the treatment of patients with left-sided breast cancer using external beam radiation therapy, Int. J. Radiat. Oncol. Biol. Phys. 56(3), 704-715 (2003).

43 Y. Negoro, Y. Nagata, T. Aoki, et al., The effectiveness of an immobilization device in conformal radiotherapy for lung tumor: Reduction of respiratory tumor movement and evaluation of the daily setup accuracy, Int. J. Radiat. Oncol. Biol. Phys. 50(4), 889-898 (2001).

44 R.C. McGarry, L. Papiez, M. Williams, T. Whitford, and R.D. Timmerman, Stereotactic body radiation therapy of early-stage non-small-cell lung carcinoma: Phase I study, Int. J. Radiat. Oncol. Biol. Phys. 63(4), 1010-1015 (2005) 
45 W. Wunderink, A. Méndez Romero, W. de Kruijf, H. de Boer, P. Levendag, and B. Heijmen, Reduction of respiratory liver tumor motion by abdominal compression in stereotactic body frame, analyzed by tracking fiducial markers implanted in liver, Int. J. Radiat. Oncol. Biol. Phys. 71(3), 907-915 (2008).

46 R. Wagman, E. Yorke, E. Ford, et al., Respiratory gating for liver tumors: Use in dose escalation, Int. J. Radiat. Oncol. Biol. Phys. 55(3), 659-668 (2003). ${ }^{47}$ S.S. Vedam, P.J. Keall, V.R. Kini, and R. Mohan, Determining parameters for respiration-gated radiotherapy, Med. Phys. 28(10), 2139-2146 (2001).

48 E. Ahunbay and X.A. Li, Investigation of the reliability, accuracy, and efficiency of gated IMRT delivery with a commercial linear accelerator, Med. Phys. 34(7), 29282938 (2007).

49 M.J. Menten, M.F. Fast, S. Nill, C.P. Kamerling, F. McDonald, and U. Oelfke, Lung stereotactic body radiotherapy with an MR-linac - Quantifying the impact of the magnetic field and real-time tumor tracking, Radiother. Oncol. 119(3), 461-466 (2016).

${ }^{50}$ M. Hoogeman, J.-B. Prévost, J. Nuyttens, J. Pöll, P. Levendag, and B. Heijmen, Clinical accuracy of the respiratory tumor tracking system of the cyberknife: assessment by analysis of log files, Int. J. Radiat. Oncol. Biol. Phys. 74(1), 297-303 (2009).

51 V. Caillet, P.J. Keall, E. Colvill, et al., MLC tracking for lung SABR reduces planning target volumes and dose to organs at risk, Radiother. Oncol. 124(1), 18-24 (2017). 
52 T. Depuydt, K. Poels, D. Verellen, et al., Treating patients with real-time tumor tracking using the Vero gimbaled linac system: implementation and first review, Radiother. Oncol. 112(3), 343-351 (2014).

53 S.S. Vedam, P.J. Keall, A. Docef, D.A. Todor, V.R. Kini, and R. Mohan, Predicting respiratory motion for four-dimensional radiotherapy, Med. Phys. 31(8), 2274-2283 (2004).

54 A.E. Lujan, J.M. Balter, and R.K. Ten Haken, A method for incorporating organ motion due to breathing into 3D dose calculations in the liver: sensitivity to variations in motion, Med. Phys. 30(10), 2643-2649 (2003).

55 S.D. McCarter and W.A. Beckham, Evaluation of the validity of a convolution method for incorporating tumour movement and set-up variations into the radiotherapy treatment planning system, Phys. Med. Biol. 45, 923-931 (2000).

56 V. Panettieri, B. Wennberg, G. Gagliardi, M.A. Duch, M. Ginjaume, and I. Lax, SBRT of lung tumours: Monte Carlo simulation with PENELOPE of dose distributions including respiratory motion and comparison with different treatment planning systems, Phys. Med. Biol. 52(14), 4265-4281 (2007).

57 I.J. Chetty, M. Rosu, D.L. McShan, B. a Fraass, J.M. Balter, and R.K. Ten Haken, Accounting for center-of-mass target motion using convolution methods in Monte Carlo-based dose calculations of the lung, Med. Phys. 31(4), 925-932 (2004).

58 T. Craig, J. Battista, and J. Van Dyk, Limitations of a convolution method for modeling geometric uncertainties in radiation therapy. I. The effect of shift invariance, 
Med. Phys. 30(8), 2001-2011 (2003).

59 K.K. Brock, D.L. McShan, R.K. Ten Haken, S.J. Hollister, L.A. Dawson, and J.M. Balter, Inclusion of organ deformation in dose calculations, Med. Phys. 30(3), 290295 (2003).

${ }^{60}$ P.J. Keall, J. V. Siebers, S. Joshi, and R. Mohan, Monte Carlo as a four-dimensional radiotherapy treatment-planning tool to account for respiratory motion, Phys. Med. Biol. 49(16), 3639-3648 (2004).

61 P.J. Keall, S. Joshi, S. Sastry Vedam, J. V. Siebers, V.R. Kini, and R. Mohan, Fourdimensional radiotherapy planning for DMLC-based respiratory motion tracking, Med. Phys. 32(4), 942-951 (2005).

62 P. J. Keall, 4-Dimensional Computed Tomography Imaging and Treatment Planning, Semin. Radiat. Oncol. 14(1), 81-90 (2004).

${ }^{63}$ P.J. Keall, G. Starkschall, H. Shukla, et al., Acquiring 4D thoracic CT scans using a multislice helical method, Phys. Med. Biol. 49(10), 2053-2067 (2004).

64 S.S. Vedam, P.J. Keall, V.R. Kini, H. Mostafavi, H.P. Shukla, and R. Mohan, Acquiring a four-dimensional computed tomography dataset using an external respiratory signal, Phys. Med. Biol. 48(1), 45-62 (2003).

65 T. Pan, Comparison of helical and cine acquisitions for 4D-CT imaging with multislice CT, Med. Phys. 32(2), 627-634 (2005).

${ }^{66}$ E. Rietzel, G.T.Y. Chen, N.C. Choi, and C.G. Willet, Four-dimensional image-based treatment planning: Target volume segmentation and dose calculation in the presence 
of respiratory motion, Int. J. Radiat. Oncol. Biol. Phys. 61(5), 1535-1550 (2005).

${ }^{67}$ T. Kleshneva, J. Muzik, and M. Alber, An algorithm for automatic determination of the respiratory phases in four-dimensional computed tomography, Phys. Med. Biol. 51(16), N269-N276 (2006).

${ }^{68}$ S.H. Jung, S.M. Yoon, S.H. Park, et al., Four-dimensional dose evaluation using deformable image registration in radiotherapy for liver cancer, Med. Phys. 40(1), 011706-1-8 (2013).

${ }^{69}$ P.R. Almond, P.J. Biggs, and W.F. Hanson, AAPM's TG - 51 Protocol for clinical reference dosimetry of high-energy photon and electron beams, Med. Phys. 26(9), 1-9 (1999).

${ }^{70}$ AAPM Report No. 87, Diode in vivo dosimetry for patients receiving external beam radiation therapy: Report of Task Group 62 of the Radiation Therapy Committee (2005).

${ }^{71}$ G. Cranmer-Sargison, S. Weston, J.A. Evans, N.P. Sidhu, and D.I. Thwaites, Monte Carlo modelling of diode detectors for small field MV photon dosimetry: detector model simplification and the sensitivity of correction factors to source parameterization, Phys. Med. Biol. 57(16), 5141-5153 (2012).

${ }^{72}$ L.J. van Battum, D. Hoffmans, H. Piersma, and S. Heukelom, Accurate dosimetry with GafChromic ${ }^{\mathrm{TM}} \mathrm{EBT}$ film of a $6 \mathrm{MV}$ photon beam in water: What level is achievable?, Med. Phys. 35(2), 704-716 (2008).

${ }^{73}$ S. Devic, Radiochromic film dosimetry: Past, present, and future, Phys. Medica 27(3), 
$122-134$ (2011).

${ }^{74}$ A. Cherpak, W. Ding, A. Hallil, and J.E. Cygler, Evaluation of a novel 4D in vivo dosimetry system, Med. Phys. 36(5), 1672-1679 (2009).

${ }^{75}$ M. Soubra and J. Cygler, Evaluation of a dual bias metal oxid-silicon semiconductor field effect transistor detector as radiation dosimeter, Med. Phys. 21(4), 567-572 (1994).

76 J.B. Hummel, M.R. Bax, M.L. Figl, et al., Design and application of an assessment protocol for electromagnetic tracking systems, Med. Phys. 32(7), 2371-2379 (2005).

${ }^{77}$ I. Kawrakow: The EGSnrc Code System: Monte Carlo Simulation of Electron and Photon Transport, Man. - Guid. NRCC Report PIRS-701 (2013).

${ }^{78}$ B.R.B. Walters, I. Kawrakow, and D.W.O. Rogers, History by history statistical estimators in the BEAM code system, Med. Phys. 29(12), 2745-2752 (2002).

79 I.J. Chetty, M. Rosu, M.L. Kessler, et al., Reporting and analyzing statistical uncertainties in Monte Carlo-based treatment planning, Int. J. Radiat. Oncol. Biol. Phys. 65(4), 1249-1259 (2006).

${ }^{80}$ D. W. O. Rogers, B. A. Faddegon, G. X. Ding, C.-M. Ma, and J. We, BEAM: A Monte Carlo code to simulate radiotherapy treatment units, Med. Phys. 22(5), 503-524 (1995).

${ }^{81}$ B. Walters, I. Kawrakow, and D.W.O. Rogers, DOSXYZnrc Users Manual, NRCC Report PIRS-0794 (2016). 
${ }^{82}$ S. Gholampourkashi, M. Vujicic, J. Belec, J.E. Cygler and E. Heath, Experimental verification of 4D Monte Carlo simulations of dose delivery to a moving anatomy, Med. Phys. 44(1), 299-310 (2017).

83 J. Lobo and I.A. Popescu, Two new DOSXYZnrc sources for 4D Monte Carlo simulations of continuously variable beam configurations, with applications to RapidArc, VMAT, TomoTherapy and CyberKnife, Phys. Med. Biol. 55(16), 4431-43 (2010).

${ }^{84}$ E. Heath and J. Seuntjens, A direct voxel tracking method for four-dimensional Monte Carlo dose calculations in deforming anatomy, Med. Phys. 33(2), 434-445 (2006).

85 J. Seco and P.M. Evans, Assessing the effect of electron density in photon dose calculations, Med. Phys. 33(2), 540-552 (2006).

86 D. A Low, W.B. Harms, S. Mutic, and J. A Purdy, A technique for the quantitative evaluation of dose distributions, Med. Phys. 25(5), 656-661 (1998).

87 I.J. Chetty, B. Curran, J.E. Cygler, et al., Report of the AAPM Task Group No. 105: Issues associated with clinical implementation of Monte Carlo-based photon and electron external beam treatment planning, Med. Phys. 34(12), 4818-4853 (2007).

${ }^{88}$ F. Verhaegen and J. Seuntjens, Monte Carlo modelling of external radiotherapy photon beams, Phys. Med. Biol. 48(21), R107-R164 (2003).

89 O. Chibani and C.M.C. Ma, On the discrepancies between Monte Carlo dose calculations and measurements for the 18 MV Varian photon beam, Med. Phys. 34(4), 1206-1216 (2007). 
90 C.M. Ma, B.A. Faddegon, D.W.O. Rogers, and T.R. Mackie, Accurate characterization of Monte Carlo calculated electron beams for radiotherapy, Med. Phys. 24(3), 401-416 (1997).

91 D. Sheikh-Bagheri, D.W. Rogers, C.K. Ross, and J.P. Seuntjens, Comparison of measured and Monte Carlo calculated dose distributions from the NRC linac, Med. Phys. 27(10), 2256-2266 (2000).

92 D. Sheikh-Bagheri and D.W.O. Rogers, Monte Carlo calculation of nine megavoltage photon beam spectra using the BEAM code, Med. Phys. 29(3), 391-402 (2002).

93 D. Sheikh-Bagheri and D.W.O. Rogers, Sensitivity of megavoltage photon beam Monte Carlo simulations to electron beam and other parameters, Med. Phys. 29(3), 379-390 (2002).

94 G.X. Ding, Energy spectra, angular spread, fluence profiles and dose distributions of 6 and $18 \mathrm{MV}$ photon beams: results of Monte Carlo simulations for a varian 2100EX accelerator, Phys. Med. Biol. 47(7), 1025-1046 (2002).

95 O. Chibani, B. Moftah, and C.-M.C. Ma, On Monte Carlo modeling of megavoltage photon beams: A revisited study on the sensitivity of beam parameters, Med. Phys. 38(1), 188-200 (2011).

96 S.S. Almberg, J. Frengen, A. Kylling, and T. Lindmo, Monte Carlo linear accelerator simulation of megavoltage photon beams: independent determination of initial beam parameters, Med. Phys. 39(1), 40-47 (2012).

97 P.J. Keall, J. V. Siebers, B. Libby, and R. Mohan, Determining the incident electron 
fluence for Monte Carlo-based photon treatment planning using a standard measured data set, Med. Phys. 30(4), 574-582 (2003).

${ }^{98}$ L.L.W. Wang and K. Leszczynski, Estimation of the focal spot size and shape for a medical linear accelerator by Monte Carlo simulation, Med. Phys. 34(2), 485-488 (2007).

99 A. Tzedakis, J.E. Damilakis, M. Mazonakis, J. Stratakis, H. Varveris, and N. Gourtsoyiannis, Influence of initial electron beam parameters on Monte Carlo calculated absorbed dose distributions for radiotherapy photon beams, Med. Phys. 31(4), 907-913 (2004).

${ }^{100} \mathrm{~K}$. Bush, S. Zavgorodni, and W. Beckham, Inference of the optimal pretarget electron beam parameters in a Monte Carlo virtual linac model through simulated annealing, Med. Phys. 36(6), 2309-2319 (2009).

${ }^{101}$ D.L. Sawkey and B.A. Faddegon, Determination of electron energy, spectral width, and beam divergence at the exit window for clinical megavoltage x-ray beams, Med. Phys. 36(3), 698-707 (2009).

${ }^{102}$ I. Chetty, J.J. DeMarco, and T.D. Solberg, A virtual source model for Monte Carlo modeling of arbitrary intensity distributions, Med. Phys. 27(1), 166-172 (2000).

${ }^{103}$ M.K. Fix, P.J. Keall, and J. V. Siebers, Photon-beam subsource sensitivity to the initial electron-beam parameters, Med. Phys. 32(4), 1164-1175 (2005).

${ }^{104}$ M. Fippel, F. Haryanto, O. Dohm, F. Nüsslin, and S. Kriesen, A virtual photon energy fluence model for Monte Carlo dose calculation, Med. Phys. 30(3), 301-311 (2003). 
${ }^{105}$ M.P. Sikora, Virtual Source Modelling of Photon Beams for Monte Carlo Based Radiation Therapy Treatment Planning, Dissertation for the degree of Philosophiae Doctor, Institute of Biomedicine, Faculty of Medicine, University of Bergen, Norway (2010).

${ }^{106}$ I. Chabert, E. Barat, T. Dautremer, et al., Development and implementation in the Monte Carlo code PENELOPE of a new virtual source model for radiotherapy photon beams and portal image calculation, Phys. Med. Biol. 61(14), 5215-5252 (2016).

${ }^{107}$ A. Ahnesjö, L. Weber, A. Murman, M. Saxner, I. Thorslund, and E. Traneus, Beam modeling and verification of a photon beam multisource model, Med. Phys. 32(6), 1722-1737 (2005).

${ }^{108}$ M. Sikora, O. Dohm, and M. Alber, A virtual photon source model of an Elekta linear accelerator with integrated mini MLC for Monte Carlo based IMRT dose calculation, Phys. Med. Biol. 52(15), 4449-4463 (2007).

${ }^{109}$ R.F. Aaronson, J.J. DeMarco, I.J. Chetty, and T.D. Solberg, A Monte Carlo based phase space model for quality assurance of intensity modulated radiotherapy incorporating leaf specific characteristics, Med. Phys. 29(12), 2952-2958 (2002).

${ }^{110}$ N. Tyagi, J.M. Moran, D.W. Litzenberg, A.F. Bielajew, B. A. Fraass, and I.J. Chetty, Experimental verification of a Monte Carlo-based MLC simulation model for IMRT dose calculation, Med. Phys. 34(2), 651-663 (2007).

${ }^{111}$ M.R. Arnfield, J. V. Siebers, J.O. Kim, Q. Wu, P.J. Keall, and R. Mohan, A method for determining multileaf collimator transmission and scatter for dynamic intensity 
modulated radiotherapy, Med. Phys. 27(10), 2231-2241 (2000).

${ }^{112}$ J. V Siebers, P.J. Keall, J.O. Kim, and R. Mohan, A method for photon beam Monte Carlo multileaf collimator particle transport, Phys. Med. Biol. 47(17), 3225-3249 (2002).

${ }^{113}$ J. Van de Walle, C. Martens, N. Reynaert, et al., Monte Carlo model of the Elekta SL $i$ plus accelerator: validation of a new MLC component module in BEAM for a 6 MV beam, Phys. Med. Biol. 48(3), 371-385 (2003).

${ }^{114}$ A.M. Bergman, E. Gete, C. Duzenli, and T. Teke, Monte Carlo modeling of HD120 multileaf collimator on Varian TrueBeam linear accelerator for verification of $6 \mathrm{X}$ and 6X FFF VMAT SABR treatment plans, J. Appl. Clin. Med. Phys. 15(3), 148-163 (2014).

${ }^{115}$ F. Crop, N. Reynaert, G. Pittomvils, et al., Monte Carlo modeling of the ModuLeaf miniature MLC for small field dosimetry and quality assurance of the clinical treatment planning system, Phys. Med. Biol. 52(11), 3275-3290 (2007).

116 J. L. Bedford, M.D.R. Thomas, and G. Smyth, Beam modeling and VMAT performance with the Agility 160-leaf multileaf collimator, J. Appl. Clin. Med. Phys. 14(2), 172-185 (2013).

${ }^{117}$ P. Kinsella, L. Shields, P. McCavana, B. McClean, and B. Langan, Determination of MLC model parameters for Monaco using commercial diode arrays, J. Appl. Clin. Med. Phys. 17(4), 37-47 (2016).

${ }^{118}$ M. Snyder, R. Halford, C. Knill, et al., Modeling the Agility MLC in the Monaco 
treatment planning system, J. Appl. Clin. Med. Phys. 17(3), 190-202 (2016).

${ }^{119}$ I.J. Das, G.X. Ding, and A. Ahnesjö, Small fields: Nonequilibrium radiation dosimetry, Med. Phys. 35(1), 206-215 (2007).

${ }^{120}$ P.H. Charles, G. Cranmer-Sargison, D.I. Thwaites, et al., A practical and theoretical definition of very small field size for radiotherapy output factor measurements, Med. Phys. 41(4), 041707 (2014).

${ }^{121}$ A.J.D. Scott, A.E. Nahum, and J.D. Fenwick, Using a Monte Carlo model to predict dosimetric properties of small radiotherapy photon fields, Med. Phys. 35(10), 46714684 (2008).

${ }^{122}$ ICRU Report 91: Prescribing, recording, and reporting of stereotactic rreatments with small photon beams, ICRU (2014).

${ }^{123}$ Institute of Physics and Engineering in Medicinie, IPEM Report 103, Small field MV photon dosimetry, IPEM (2010).

${ }^{124}$ A.J.D. Scott, A.E. Nahum, and J.D. Fenwick, Monte Carlo modeling of small photon fields: Quantifying the impact of focal spot size on source occlusion and output factors, and exploring miniphantom design for small-field measurements, Med. Phys. 36(7), 3132-3144 (2009).

${ }^{125}$ R. Capote, F. Sánchez-Doblado, A. Leal, J.I. Lagares, R. Arráns, and G.H. Hartmann, An EGSnrc Monte Carlo study of the microionization chamber for reference dosimetry of narrow irregular IMRT beamlets, Med. Phys. 31(9), 2416-2422 (2004).

${ }^{126}$ D. Czarnecki and K. Zink, Monte Carlo calculated correction factors for diodes and 
ion chambers in small photon fields, Phys. Med. Biol. 59(3), 791-794 (2014).

${ }^{127}$ J.Y. Cheng, H. Ning, B.C. Arora, Y. Zhuge, and R.W. Miller, Output factor comparison of Monte Carlo and measurement for Varian TrueBeam $6 \mathrm{MV}$ and $10 \mathrm{MV}$ flattening filter-free stereotactic radiosurgery system, J. Appl. Clin. Med. Phys. 17(3), 100-110 (2016).

${ }^{128}$ P. Andreo, H. Palmans, M. Marteinsdóttir, H. Benmakhlouf, and Å. CarlssonTedgren, On the Monte Carlo simulation of small-field micro-diamond detectors for megavoltage photon dosimetry, Phys. Med. Biol. 61(1), L1-L10 (2016).

${ }^{129}$ K. Paskalev, J.P. Seuntjens, H.J. Patrocinio, and E.B. Podgorsak, Physical aspects of dynamic stereotactic radiosurgery with very small photon beams $(1.5$ and $3 \mathrm{~mm}$ in diameter), Med. Phys. 30(2), 111-118 (2003).

${ }^{130}$ F. Araki, Monte Carlo study of a Cyberknife stereotactic radiosurgery system, Med. Phys. 33(8), 2955-2963 (2006).

${ }^{131}$ D. W. O. Rogers, B. Walters, and I. Kawrakow, BEAMnrc User's Manual, NRCC Report PIRS-0509, (2013).

${ }^{132}$ M. Fippel, Fast Monte Carlo dose calculation for photon beams based on the VMC electron algorithm, Med. Phys. 26(8), 1466-1475 (1999).

${ }^{133}$ I. Kawarkow, M. Fippel, and K. Friedrich, 3D electron dose calculation using a Voxel based Monte Carlo algorithm (VMC), Med. Phys. 23(4), 445-457 (1996).

${ }^{134}$ N. Kostiukhina, D. Georg, S. Rollet, et al., Advanced Radiation DOSimetry phantom (ARDOS): A versatile breathing phantom for 4D radiation therapy and medical 
imaging, Phys. Med. Biol. 62(20), 8136-8153 (2017).

${ }^{135}$ S. Pallotta, S. Calusi, L. Foggi, et al., ADAM: A breathing phantom for lung SBRT quality assurance, Phys. Medica 49, 147-155 (2018).

${ }^{136}$ A.C. Vàsquez, A. Runz, G. Echner, G. Sroka-Perez, and C.P. Karger, Comparison of two respiration monitoring systems for 4D imaging with a Siemens CT using a new dynamic breathing phantom, Phys. Med. Biol. 57(9), 131-143 (2012).

${ }^{137}$ W. Ottosson, C.F. Behrens, and C.E. Andersen, Dose verification of radiotherapy for lung cancer by using plastic scintillator dosimetry and a heterogeneous phantom, J. Phys. Conf. Ser. 573(1), 3-7 (2015).

${ }^{138}$ R. Kashani, K. Lam, D. Litzenberg, and J. Balter, Technical note: A deformable phantom for dynamic modeling in radiation therapy, Med. Phys. 34(1), 199-201 (2007).

${ }^{139}$ R. Kashani, M. Hub, M.L. Kessler, and J.M. Balter, Technical note: A physical phantom for assessment of accuracy of deformable alignment algorithms, Med. Phys. 34(7), 2785-2788 (2007).

${ }^{140}$ Y. Cheung and A. Sawant, An externally and internally deformable, programmable lung motion phantom, Med. Phys. 42(5), 2585-2593 (2015).

${ }^{141}$ M. Szegedi, P. Rassiah-Szegedi, G. Fullerton, B. Wang, and B. Salter, A prototype design of a real-tissue phantom for the validation of deformation algorithms and 4D dose calculations, Phys. Med. Biol. 55(13), 3685-3699 (2010).

${ }^{142}$ G. Remmert, J. Biederer, F. Lohberger, M. Fabel, and G.H. Hartmann, Four- 
dimensional magnetic resonance imaging for the determination of tumour movement and its evaluation using a dynamic porcine lung phantom, Phys. Med. Biol. 52(18), N401-N415 (2007).

${ }^{143}$ J. Chang, T.S. Suh, and D.S. Lee, Development of a deformable lung phantom for the evaluation of deformable registration, J. Appl. Clin. Med. Phys. 11(1), 281-286 (2010).

${ }^{144}$ D. Shin, S. Kang, D. Kim, et al., Development of an advanced deformable phantom to analyze dose differences due to respiratory motion, Prog. Med. Phys., 28(1), 1-10 (2017).

${ }^{145}$ J. Kim, Y. Lee, H. Shin, et al., Development of deformable moving lung phantom to simulate respiratory motion in radiotherapy, Med. Dosim. 41(2), 113-117 (2016).

${ }^{146}$ E. Nioutsikou, J. R. N. Symonds-Tayler, J.L. Bedford, and S. Webb, Quantifying the effect of respiratory motion on lung tumour dosimetry with the aid of a breathing phantom with deforming lungs, Phys. Med. Biol. 51(14), 3359-3374 (2006).

${ }^{147}$ M. Serban, E. Heath, G. Stroian, D.L. Collins, and J. Seuntjens, A deformable phantom for 4D radiotherapy verification: design and image registration evaluation., Med. Phys. 35(3), 1094-1102 (2008).

${ }^{148}$ A. Cherpak, M. Serban, J. Seuntjens, and J.E. Cygler, 4D dose-position verification in radiation therapy using the RADPOS system in a deformable lung phantom, Med. Phys. 38(1), 179-187 (2011).

${ }^{149}$ L.E. Court, J. Seco, X.-Q. Lu, et al., Use of a realistic breathing lung phantom to 
evaluate dose delivery errors, Med. Phys. 37(11), 5850-5857 (2010).

${ }^{150}$ P. Mann, M. Witte, T. Moser, et al., 3D dosimetric validation of motion compensation concepts in radiotherapy using an anthropomorphic dynamic lung phantom, Phys. Med. Biol. 62(2), 573-595 (2017).

${ }^{151}$ Y.Y. Vinogradskiy, P. Balter, D.S. Followill, P.E. Alvarez, R.A. White, and G. Starkschall, Verification of four-dimensional photon dose calculations, Med. Phys. 36(8), 3438-47 (2009).

${ }^{152}$ M. Rosu, I.J. Chetty, J.M. Balter, M.L. Kessler, D.L. McShan, and R.K. Ten Haken, Dose reconstruction in deforming lung anatomy: dose grid size effects and clinical implications, Med. Phys. 32(8), 2487-2495 (2005).

${ }^{153}$ M. Ding, J. Li, J. Deng, E. Fourkal, and C.M. Ma, Dose correlation for thoracic motion in radiation therapy of breast cancer, Med. Phys. 30(9), 2520-2529 (2003).

${ }^{154}$ B. Schaly, J. A. Kempe, G.S. Bauman, J.J. Battista, and J. Van Dyk, Tracking the dose distribution in radiation therapy by accounting for variable anatomy, Phys. Med. Biol. 49(5), 791-805 (2004).

${ }^{155}$ H. Paganetti, H. Jiang, J. A. Adams, G.T. Chen, and E. Rietzel, Monte Carlo simulations with time-dependent geometries to investigate effects of organ motion with high temporal resolution, Int. J. Radiat. Oncol. Biol. Phys. 60(3), 942-950 (2004).

${ }^{156}$ J. V. Siebers and H. Zhong, An energy transfer method for 4D Monte Carlo dose calculation, Med. Phys. 35(9), 4096-4105 (2008). 
${ }^{157}$ H. Zhong and J. V. Siebers, Monte Carlo dose mapping on deforming anatomy, Phys. Med. Biol. 54, 5815-5830 (2009).

${ }^{158}$ N. Stanley, C. Glide-Hurst, J. Kim, et al., Using patient-specific phantoms to evaluate deformable image registration algorithms for adaptive radiation therapy, J. Appl. Clin. Med. Phys. 14(6), 177-194 (2013).

${ }^{159}$ N. Kirby, C. Chuang, and J. Pouliot, A two-dimensional deformable phantom for quantitatively verifying deformation algorithms, Med. Phys. 38(8), 4583-4586 (2011).

${ }^{160}$ E. Heath, D.L. Collins, P.J. Keall, L. Dong, and J. Seuntjens, Quantification of accuracy of the automated nonlinear image matching and anatomical labeling (ANIMAL) nonlinear registration algorithm for 4D CT images of lung, Med. Phys. 34(11), 4409-4421 (2007).

${ }^{161}$ H. Zhong, J. Adams, C. Glide-Hurst, H. Zhang, H. Li, and I. Chetty, Development of a deformable dosimetric phantom to verify dose accumulation algorithms for adaptive radiotherapy, J. Med. Phys. 41(2), 106-114 (2016).

${ }^{162}$ F. Liu, Y. Hu, Q. Zhang, R. Kincaid, K.A. Goodman, and G.S. Mageras, Evaluation of deformable image registration and a motion model in CT images with limited features, Phys. Med. Biol. 57(9), 2539-2554 (2012).

${ }^{163}$ J. Bélec and B.G. Clark, Monte Carlo calculation of VMAT and helical tomotherapy dose distributions for lung stereotactic treatments with intra-fraction motion, Phys. Med. Biol. 58(9), 2807-21 (2013).

${ }^{164}$ J. Bélec, N. Ploquin, D.J. La Russa, and B.G. Clark, Position-probability-sampled 
Monte Carlo calculation of VMAT, 3DCRT, step-shoot IMRT, and helical tomotherapy dose distributions using BEAMnrc/DOSXYZnrc, Med. Phys. 38(2), 948$960(2011)$.

${ }^{165}$ Y.Y. Vinogradskiy, P. Balter, D.S. Followill, P.E. Alvarez, R.A. White, and G. Starkschall, Comparing the accuracy of four-dimensional photon dose calculations with three-dimensional calculations using moving and deforming phantoms, Med. Phys. 36(11), 5000-6 (2009).

${ }^{166}$ C.J. Niu, W.D. Foltz, M. Velec, J.L. Moseley, A. Al-Mayah, and K.K. Brock, A novel technique to enable experimental validation of deformable dose accumulation, Med. Phys. 39(2), 765-776 (2012).

${ }^{167}$ M. McEwen, L. DeWerd, G. Ibbott, et al., Addendum to the AAPM's TG-51 protocol for clinical reference dosimetry of high-energy photon beams, Med. Phys. 41(4), 041501-20 (2014).

${ }^{168}$ J. Seuntjens, M. Olivares, M. Evans, and E. Podgorsak, Absorbed dose to water reference dosimetry using solid phantoms in the context of absorbed-dose protocols, Med. Phys. 32(9), 2945-2953 (2005).

${ }^{169}$ N. Papanikolaou, T.R. Mackie, C. Meger-Wells, M. Gehring, and P. Reckwerdt, Investigation of the convolution method for polyenergetic spectra, Med. Phys. 20(5), $1327-1336(1993)$.

${ }^{170}$ J.D. Lawson, E. Schreibmann, A.B. Jani, and T. Fox, Quantitative evaluation of a cone-beam computed tomography - planning computed tomography deformable image 
registration method for adaptive radiation therapy, J. Appl. Clin. Med. Phys. 8(4), 96113 (2007). 